\title{
Urban Wake Field Generation Using LES for Application to Quadrotor Flight
}

\author{
by \\ Mark Sutherland \\ A Thesis submitted to \\ the Faculty of Graduate Studies and Research \\ in partial fulfilment of \\ the requirements for the degree of \\ Master of Applied Science \\ in
}

\author{
Aerospace Engineering \\ Department of Mechanical and Aerospace Engineering \\ Carleton University \\ Ottawa, Ontario, Canada
}

May 2015

Copyright ()

2015 - Mark Sutherland 


\section{Abstract}

A method is presented for using LES to generate urban wake fields for use in studying their effects on autonomous quadrotor's flight performance. The flow field is generated around a single square building using OpenFOAM, and a MATLAB/Simulink flight simulator is used to compare the difference in flight performance between wake fields generated by RANS and LES. It is found LES causes maximum position deviations 2 orders of magnitude larger compared to RANS, and results in skewed deviations by as much as 5 to 1 in a given direction. Since the transient turbulent LES wake field more accurately reflects the flow present behind real world structures, LES generated wake fields should be used when designing and testing autonomous control algorithms for multirotor UAVs on the order of $0.5 \mathrm{~m}$ in size and $2 \mathrm{~kg}$ in mass. 


\section{Acknowledgments}

Without question I would like to thank my supervisor Professor Jason Etele of Carleton University for providing such a unique opportunity and unparalleled guidance. Little did I know your patience, trust, feedback, and humor would make this endeavor incredibly pleasant, dare I say, fun.

Similarly, thank you to Dr. Giovanni Fusina of Defense Research and Development Canada for the chance to work on such a project and the consequent large amount of leeway and freedom on it.

Certainly a resounding thank you to friends and family for your wonderful support leading up to and during this enterprise.

Finally to new and old friends alike in Ottawa, you have made my time here, and overall experience, just that much more enjoyable.

Thank you to everyone. 


\section{Table of Contents}

$\begin{array}{ll}\text { Abstract } & \text { ii }\end{array}$

Acknowledgments

List of Tables vii

List of Figures viii

List of Acronyms xii

List of Symbols $\quad$ xiv

1 Introduction 1

1.1 Motivation . . . . . . . . . . . . . . . . . . 1

1.2 Brief UAS History $\ldots \ldots \ldots \ldots \ldots$

1.3 Wind Modeling and Applications $\ldots \ldots \ldots \ldots \ldots$

1.4 Urban Environment . . . . . . . . . . . . . . . . . . . . . . . 12

2 Methodology 15

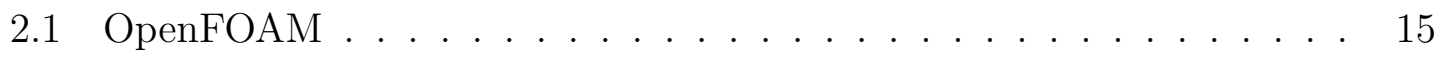


2.2 Urban Wake Field CFD Modeling . . . . . . . . . . . . . . . . . 20

2.3 Reynolds-Averaged Navier-Stokes Method . . . . . . . . . . . . . . 21

2.4 Large-Eddy Simulation Method . . . . . . . . . . . . . . 26

3 Grid Refinement Study 36

3.1 Computational Domain . . . . . . . . . . . . . . . . . 36

3.2 LES Verification and Validation Study . . . . . . . . . . . . . . 39

3.2.1 Q-Criterion Turbulence Visualization . . . . . . . . . . . 41

3.2.2 Time Averaged Velocity Profile . . . . . . . . . . . . . . 43

3.2 .3 LES Index Quality . . . . . . . . . . . . . . . . . . . 47

3.2.4 Experimental Comparison . . . . . . . . . . . . . 50

3.3 RANS Wake Field . . . . . . . . . . . . . . . . . . 53

4 Simulation Methodology $\quad 55$

4.1 Urban Wake Database . . . . . . . . . . . . . . . 55

4.1.1 Temporal Resolution Study .............. 56

4.1.2 Database Loop Interval . . . . . . . . . . . . . . . . 58

4.2 Flight Controller . . . . . . . . . . . . . . . . . 61

$\begin{array}{llr}5 & \text { Results } & 76\end{array}$

5.1 Mission 1 - Freestream Wind Position Hold . . . . . . . . . . . . . . . 80

5.2 Mission 2 - Building Wake Position Hold . . . . . . . . . . . . . . . . 84

5.3 Mission 3 - Top Wake Boundary Position Hold . . . . . . . . . . . . . 88

5.4 Mission 4 - Ascent Through Wake . . . . . . . . . . . . . . 91

5.5 Mission 5 - Crosswind Wake Translation . . . . . . . . . . . . . 94 
6 Conclusions and Recommendations $\quad 98$

6.1 Conclusions . . . . . . . . . . . . . . . . . . . . . . . 98

6.2 Recommendations . . . . . . . . . . . . . . . . . 100

$\begin{array}{ll}\text { List of References } & 102\end{array}$

Appendix A LES Standard Working Directory 111

Appendix B Using OpenFOAM on Kumomotojo 120

B.1 Using OpenFOAM . . . . . . . . . . . . . . . . . 120

B.2 Using ParaView . . . . . . . . . . . . . . . . . . 123

B.2.1 Configure Linux Client . . . . . . . . . . . . . . . . . . . 124

B.2.2 Configure Windows Client . . . . . . . . . . . . . . 125

B.2.3 Results Viewing . . . . . . . . . . . . . . . . 131

Appendix C Urban Wake Database 134

Appendix D Database-Simulator Integration 141

D.1 MySQL Database Structure . . . . . . . . . . . . . . . . 142

D.2 SSH Tunnel . . . . . . . . . . . . . . . . . . 143

D.2.1 Configure Linux Client . . . . . . . . . . . . . . . 143

D.2.2 Configure Windows Client . . . . . . . . . . . . . . . 143

D.3 Driver Installation . . . . . . . . . . . . . . . . . . 145

D.4 Database Access and MATLAB Connection . . . . . . . . . . . . 146 


\section{List of Tables}

1 Refined area grid details . . . . . . . . . . . . . . . . 39

2 Urban wind database query time $\ldots \ldots \ldots \ldots \ldots$

3 Quadrotor flight simulator parameters . . . . . . . . . . . 72

4 Position controller PD gains . . . . . . . . . . . . . . . . 74

5 Attitude controller PID gains . . . . . . . . . . . . . 74 


\section{List of Figures}

1 Sample fixed-wing unmanned aircraft . . . . . . . . . . . . 3

2 Sample rotor-craft unmanned aircraft . . . . . . . . . . . . 4

3 Sample small unmanned aircraft . . . . . . . . . . . . . 5

4 Evolution of multirotor aircraft . . . . . . . . . . . . . . 6

5 Typical multirotor configurations . . . . . . . . . . . . . 7

6 Ideal atmospheric boundary layer due an urban area . . . . . . . . . 8

$7 \quad$ Primary building block parameters . . . . . . . . . . . . . . . . 13

8 Sample of used OpenFOAM solver and utilities libraries. . . . . . . . 16

9 Two corrector step PISO algorithm . . . . . . . . . . . . . . . 17

10 Outline of the standard LES working directory . . . . . . . . . . . . 19

11 Reynolds decomposition of $\Phi$ over time . . . . . . . . . . . . . . . . . 21

12 Turbulent kinetic energy spectrum with LES cutoff width . . . . . . . 27

13 LES decomposition and filter properties . . . . . . . . . . . . 29

14 Common LES filter function . . . . . . . . . . . . . . . . . . . . 30

15 Box filter example using DNS velocity field . . . . . . . . . . . . . 31

16 Computational domain . . . . . . . . . . . . . . . . . 37

17 Wake region Cartesian hex mesh . . . . . . . . . . . . . . . 38 
18 Data sample and plotting locations . . . . . . . . . . . . . . 40

19 Q-criterion vortex visualization $\ldots \ldots \ldots \ldots \ldots \ldots$

20 Time averaged streamwise velocity profiles, $x / R_{\|}=0.75 \ldots \ldots .44$

21 Time averaged streamwise velocity profiles, $x / R_{\|}=1.25 \ldots \ldots \ldots$

22 Time averaged streamwise velocity profiles, $x / R_{\|}=2.00 \ldots \ldots .47$

$23 L E S \_I Q_{k}, x / R_{\|}=0.75 \ldots \ldots \ldots \ldots \ldots \ldots$

$24 L E S \_I Q_{k}, x / R_{\|}=1.25 \ldots \ldots \ldots \ldots \ldots \ldots$

$25 L E S \_I Q_{k}, x / R_{\|}=2.00 \ldots \ldots \ldots \ldots \ldots \ldots \ldots$

26 Experimental vs. numerical comparison, $x / R_{\|}=0.75 \ldots \ldots \ldots$

27 Experimental vs. numerical comparison, $x / R_{\|}=1.25 \ldots \ldots \ldots$

28 Experimental vs. numerical comparison, $x / R_{\|}=2.00 \ldots \ldots \ldots . \quad 52$

29 RANS and LES streamwise velocity profiles, $x / R_{\|}=1.25 \ldots \ldots \ldots 54$

30 LES wake database timestep resolution study $\ldots \ldots \ldots$. . . . . 57

31 Total velocity contours for database loop interval estimation . . . . . 59

32 Database loop crosswind force coefficient and probed velocity . . . . . 60

33 Quadrotor attitude and position control . . . . . . . . . . . . 62

34 Inertial and body fixed reference frames . . . . . . . . . . . . . 63

35 Quadrotor free body diagram and notation convention . . . . . . 65

36 Quadrotor cascade attitude and position control method . . . . . . 73

37 Quadrotor cascade PD-PID control block diagram . . . . . . . . . 75

38 Flight mission locations and velocity field samples . . . . . . . . . 77

39 Flight mission locations and velocity field samples . . . . . . . . 78

40 Mission 1 - wind velocity components . . . . . . . . . . . . . 81

41 Mission 1 - quadrotor position . . . . . . . . . . . . . . 82 
42 Mission 1 - maximum path deviation bounding boxes . . . . . . . . . 83

43 Mission 2 - wind velocity components . . . . . . . . . . . . . 85

44 Mission 2 - quadrotor position . . . . . . . . . . . . 86

45 Mission 2 - maximum path deviation bounding boxes . . . . . . . 87

46 Mission 3 - wind velocity components . . . . . . . . . . . . . . . 88

47 Mission 3 - quadrotor position . . . . . . . . . . . . . . . . 89

48 Mission 3 - maximum path deviation bounding boxes . . . . . . . . . 90

49 Mission 4 - LES deviations and building . . . . . . . . . . . . . 91

50 Mission 4 - wind velocity components . . . . . . . . . . . . . . 93

51 Mission 4 - quadrotor position . . . . . . . . . . . . . . 94

52 Mission 5 - LES deviations and building . . . . . . . . . . . . 95

53 Mission 5 - wind velocity components . . . . . . . . . . . . 96

54 Mission 5 - quadrotor position . . . . . . . . . . . . . 97

55 OpenFOAM LES user workflow . . . . . . . . . . . . . . . 111

56 Outline of the standard OpenFOAM working directory . . . . . . . . 112

57 icoFoam usage output with OpenFOAM version . . . . . . . . . . . 121

58 icoFoam function not found message . . . . . . . . . . . . . 121

59 SSH tunnel Putty setup . . . . . . . . . . . . . . 125

60 Putty connection setup . . . . . . . . . . . . . . . 126

61 Connect to server selection . . . . . . . . . . . . . . . . . . . . 129

62 Add new server . . . . . . . . . . . . . . . . . 129

63 Enter the server details . . . . . . . . . . . . . . . . . 130

64 Select Manual and Save . . . . . . . . . . . . . . . . . . 130

65 Connect to server selection . . . . . . . . . . . . . . . 131 
66 Server selection . . . . . . . . . . . . . . . . 132

67 Server connection and open a case . . . . . . . . . . . . . . . . 133

68 Remote ParaView workflow, dashed: client actions, solid: server actions133

69 Load run.foam file, adjust timestep and select desired variables . . . . 135

70 Save data and set file prefix . . . . . . . . . . . . . . 135

71 Save the data from the points and for all timesteps . . . . . . . . 136

72 Single building characteristics . . . . . . . . . . . . . . . . 142

73 SSH tunnel Putty setup . . . . . . . . . . . . . . . . 144

74 Putty connection setup . . . . . . . . . . . . . . . . 145

75 List of all the databases stored on the MySQL server . . . . . . . . . 147

76 List of tables in windDB . . . . . . . . . . . . . . . . . . 148

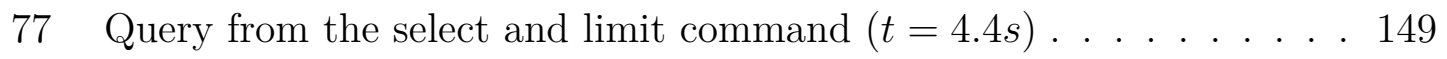




\section{List of Acronyms}

\begin{tabular}{ll}
\hline Acronyms & Definition \\
\hline ABL & Atmospheric Boundary Layer \\
AIJ & Agriculture Institute of Japan \\
CFD & Computation fluid dynamics \\
CG & Center of gravity \\
CLF & Courant-Friedrichs-Lewy \\
ESC & Electronic Speed Controller \\
FAA & Federal Aviation Authority \\
GCS & Ground Control Station \\
GPS & Global Positioning System \\
GUI & Graphical User Interface \\
IMU & Inertial Measurement Unit \\
LES & Large-Eddy Simulation \\
LiDAR & Light Direction And Ranging \\
MUA & Micro Unmanned Aircraft \\
\end{tabular}




\begin{tabular}{ll} 
NED & North-East-Down \\
OEE & One-Equation Eddy \\
PID & Proportional-Integral-Derivative \\
PISO & Pressure Implicit with Splitting of Operators \\
PWM & Pulse Width Modulation \\
RANS & Reynolds-Averaged Navier-Stokes \\
RPA & Remotely Piloted Aircraft \\
RSM & Reynolds Stress Model \\
SGS & Sub-grid scale \\
SGMV & Systematic Grid and Model Verification \\
SIMPLE & Semi-Implicit Method for Pressure-Linked Equations \\
SST & Shear Stress Transport \\
SUA & Small Unmanned Aircraft \\
TKE & Turbulent Kinetic Energy \\
UA & Unmanned Aircraft \\
UAS & Unmanned Aerial System \\
UAV & Unmanned Aerial Vehicle \\
UCA & Unmanned Combat Aircraft \\
UWD & Urban wind database \\
VTOL & Vertical Take off and Landing \\
\hline &
\end{tabular}




\section{List of Symbols}

\begin{tabular}{lll}
\hline Symbols & Definition & Units \\
\hline$F$ & Force & $\mathrm{kg} /\left(\mathrm{m} \cdot \mathrm{s}^{2}\right)$ \\
$G$ & LES filter kernel & $\mathrm{m}$ \\
$h$ & Characteristic grid quality & $\mathrm{m}$ \\
$H$ & Building height & \\
$I$ & Inertia matrix & $\mathrm{m}^{2} / \mathrm{s}^{2}$ \\
$k$ & Specific kinetic energy & \\
$K$ & Control gain & \\
$L$ & Rotation matrix & $\mathrm{kg}$ \\
$m$ & Mass & \\
$M$ & Quadrotor motor & \\
$M$ & Moment vector & $\mathrm{m}^{2} / \mathrm{s}^{2}$ \\
$p$ & Pressure (density nominalized) & $\mathrm{rad} / \mathrm{s}$ \\
$p$ & Roll rate & $\mathrm{rad} / \mathrm{s}$ \\
$q$ & Pitch rate & \\
$r$ & Cell centered local reference frame &
\end{tabular}

xiv 


\begin{tabular}{|c|c|c|}
\hline$r$ & Yaw rate & $\mathrm{rad} / \mathrm{s}$ \\
\hline$R$ & Building side dimension & $m$ \\
\hline$R e$ & Reynolds number & \\
\hline$S$ & Surface area & \\
\hline$S$ & Strain-rate tensor & \\
\hline$S t$ & Strouhal number & \\
\hline$t$ & Time & $s$ \\
\hline$T$ & Time average window & $s$ \\
\hline$T$ & Thrust & $k g /\left(m \cdot s^{2}\right)$ \\
\hline$u$ & Velocity component & $m / s$ \\
\hline$U$ & Total uncertainty & \\
\hline$U$ & Control output & \\
\hline$V$ & Total velocity & $m / s$ \\
\hline$W$ & Wind velocity & $m / s$ \\
\hline$x$ & Streamwise co-ordinate direction & \\
\hline$y$ & Cross wind co-ordinate direction & \\
\hline$z$ & Vertical co-ordinate direction & \\
\hline$X$ & x co-ordinate position & $m$ \\
\hline$Y$ & y co-ordinate position & $m$ \\
\hline$Z$ & z co-ordinate position & $m$ \\
\hline$\delta$ & Kronecker delta & \\
\hline$\Delta$ & LES filter width & $m$ \\
\hline$\kappa$ & Von Karman constant & \\
\hline
\end{tabular}




$\begin{array}{lll}\mu & \text { Dynamic viscosity } & \mathrm{kg} /(\mathrm{m} \cdot \mathrm{s}) \\ \nu & \text { Kinematic viscosity } & \mathrm{m}^{2} / \mathrm{s} \\ \varepsilon & \text { Error } & \\ \phi & \text { Specific kinetic energy dissipation rate } & \mathrm{m}^{2} / \mathrm{s}^{3} \\ \Phi & \text { Roll angle } & \\ \psi & \text { Flow variable } & \\ \tau & \text { Yaw angle } & \mathrm{m}^{2} / \mathrm{s}^{2} \\ \theta & \text { Stress tensor } & \\ \omega & \text { Pitch angle } & 1 / \mathrm{s} \\ \zeta & \text { Angular velocity } & \circ\end{array}$

Accents

$\begin{array}{ll}\cdot & \text { Rate } \\ - & \text { Mean } \\ \sim & \text { Filtered } \\ \rightarrow & \text { Vector }\end{array}$

Superscript

I

Fluctuating/SGS component

res Resolved

tot Total

$p \quad$ Numerical accuracy order

Subscript

\| $\quad$ Wind parallel 


\begin{tabular}{ll}
$\perp$ & Wind perpendicular \\
0 & Effective surface roughness \\
$*$ & Friction velocity \\
$B$ & Body frame \\
$c$ & Filter cutoff width \\
$d$ & Drag \\
$D$ & Characteristic building length \\
$E$ & Earth frame \\
$f$ & Force \\
$g$ & Gravity \\
$i$ & Co-ordinate direction \\
$j$ & Co-ordinate direction \\
$k$ & Richardson constant \\
$m$ & Mean \\
$S G S$ & Sub-grid scale \\
$t$ & Turbulent \\
$W$ & Wind \\
$x$ & Streamwise co-ordinate direction \\
$y$ & Cross wind co-ordinate direction \\
$z$ & Vertical co-ordinate direction \\
$\infty$ & Freestream \\
\hline &
\end{tabular}

xvii 


\section{Chapter 1}

\section{Introduction}

\subsection{Motivation}

The development and utilization of unmanned aerial vehicles (UAVs) has dramatically increased over the last decade as a result of various global conflicts and ease of public access. A quick distinction is made here between an aerial munition such as a cruise missile and a UAV, as the former is a one-time use weapon while the latter is designed to be reused and perform various types of missions. As the complexity grew, the term UAV became outdated and was enhanced with the concept of an unmanned aerial system (UAS), designed to incorporate the unmanned aircraft (UA), ground control station (GCS), command and communication data links,

and any additional required system elements [1]. Further classification of UAs is made with subcategories for remotely piloted aircraft (RPA) and fully autonomous navigation and control systems. 
With an estimated $53 \%$ of the worlds population living in an urban environment, and increasing $2 \%$ annually [2], there is little surprise in the growing interest in using UAS's in urban environments. While both fixed-wing and rotor-craft platforms are versatile and capable for use in an urban environment, the focus of this work is on a quadrotor. This UAS is very useful for many different mission profiles such as; law enforcement [3], general reconnaissance and surveillance [4], aerial photography for building inspection [5], urban mapping, first responses tool [6], forensic analysis [7], traffic camera, chemical sensors, parcel delivery $[8,9]$, and catering services $[10,11]$.

However, the size and weight of vehicles able to operate within an urban environment are susceptible to external environmental effects such turbulent wakes produced by urban structures. This external susceptibility of aircraft flight in urban environments is outlined in the previous work of Etele [12] and Galway et al. [13-15], as well as other studies such as Orr et al. [16], Kothari et al. [17], and Semsch et al. [18].

The resulting random transient wind forces generated in a building's wake can cause significant trajectory and pathing deviations for both RPA or fully autonomous unmanned systems. To overcome this environmental influence two main branches of study have formed; design a controller to estimate the wind's external influence and counteract it, as outlined by Waslander et al. [19], or design a robust intelligent controller to dynamically adapt to the external fores, as used by Raza et al. [20]. The work presented here complements the design and simulated testing of robust autonomous control algorithms by generating appropriate and representative urban wake fields. 


\subsection{Brief UAS History}

The usefulness of any UAS becomes apparent when considering the additional possible mission types otherwise too mundane, dangerous, or time consuming for piloted aircraft. Therefore, similar to their 'full size' counterparts both fixed-wing and rotorcraft UA platforms have been developed to make use of the different advantages for various mission profiles. One of the most iconic fixed-wing unmanned combat aircraft (UCA), an armed subclass as a UA, is General Atomics MQ-1 Predator as shown in Figure 1a. In addition to removing valuable pilots from combat situations, the UAS excels in information gathering for reconnaissance mission profiles. The realization for such a platform came at height of the Cold War when prior to spy satellites the U-2 spy plane was primary means of reconnaissance. After the Soviets shot down the U-2s of Francis Gary Powers in 1960 and Rudolf Anderson, Jr. in 1962, it became clear an unmanned intelligence gathering vehicle could be beneficial. This ultimately culminated in the development of the recent generation platforms such of the Northrop Grumman MQ-4C Triton surveillance UA pictured in Figure $1 b$.

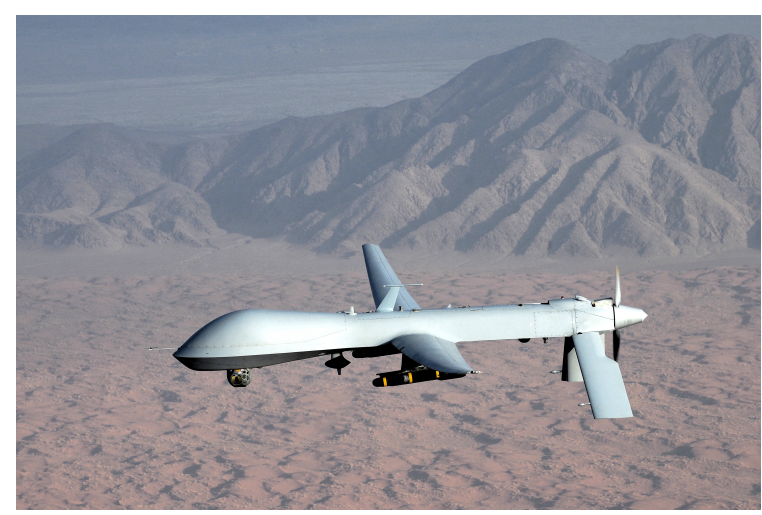

(a) MQ-1 Predator [21]

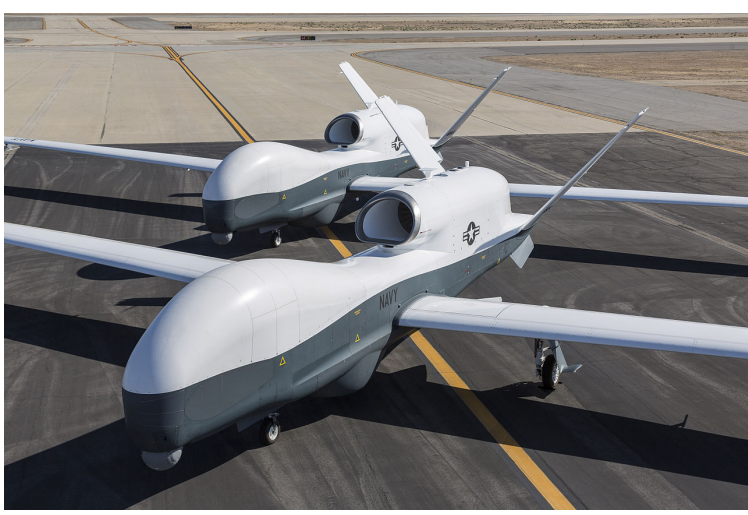

(b) MQ-4C Triton [22]

Figure 1: Sample fixed-wing unmanned aircraft 
While not as common as the fixed-wing platforms, various rotor-craft UAs have been developed to take advantage of the rotor-craft's vertical take off and landing (VTOL) capabilities. This allows for simpler vehicle deployment off smaller navel vessels and highly localized reconnaissance or engagement of a desired area. The Northrop Grumman MQ-8 Fire Scout and Schiebel Camcopter S-100, shown in Figures 2a and $2 \mathrm{~b}$ respectively, are examples of rotor-craft UAs developed to fit such mission types.

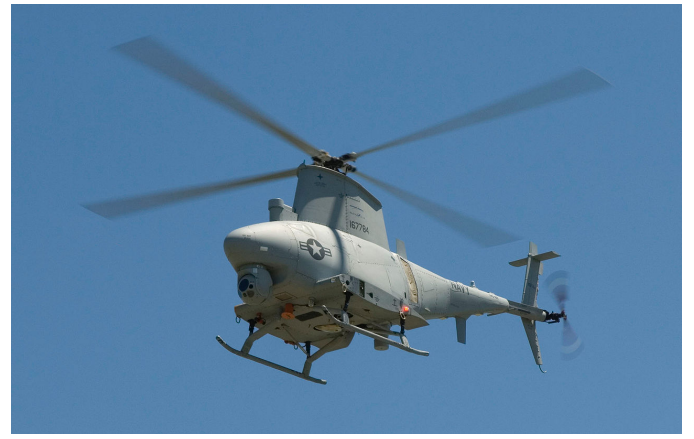

(a) MQ-8 Fire Scout [23]

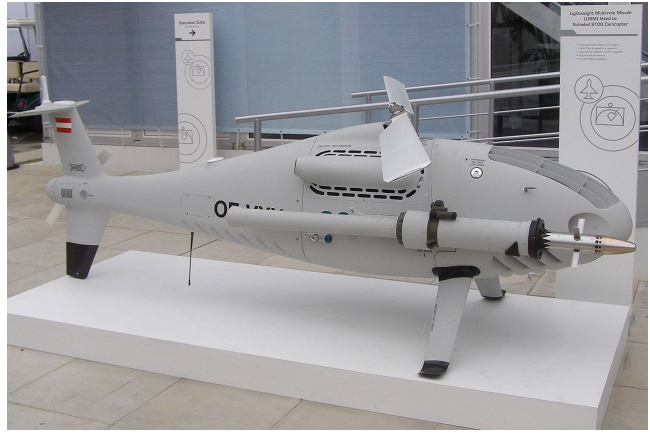

(b) S-100 Camcopter [24]

Figure 2: Sample rotor-craft unmanned aircraft

The continued success and development of various UAS platforms, coupled with advances in computational hardware, has lead to UA miniaturization resulting in the small unmanned aircraft (SUA). This wide general classification includes a range of vehicles from human portable miniature UAs to insect sized micro unmanned aircraft (MUA). Lower development and production costs, an overall simpler systems requirements, and ease of hardware access have lead to an incredible increase in the research, advancement, and implementation of SUAs. This boom of interest has occurred in a multitude of different sectors such as the military, academics, industry, and hobbyists. Two examples of the numerous available SUAs are the fixed-wing EMT Aladin and quadrotor Aeryon Scout illustrated in Figures 3a and $3 \mathrm{~b}$ respectively. 


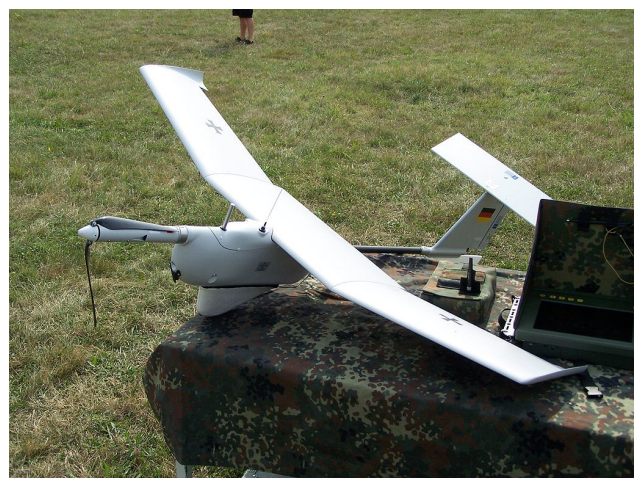

(a) EMT Aladin [25]

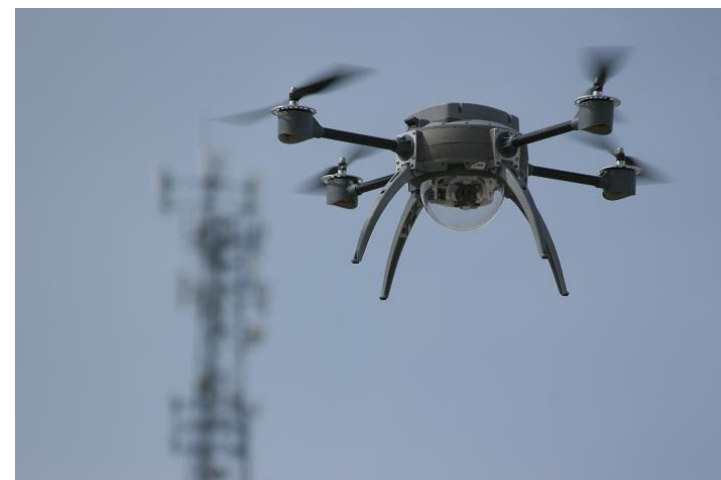

(b) Aeryon Scout [26]

Figure 3: Sample small unmanned aircraft

With comparably less flight time available in regards to their 'full size' equivalents, the size and cost of the small UASs still opens opportunities not previously available leading to their use in numerous industrial and civilian applications such as, precision agriculture, geological or mine site surveying, forest fire examination, or remote search and rescue operations.

Mutlirotor aircraft designs have existed since the Breguet-Richet Gryoplane in 1907 and the de Bothezat helicopter in 1922, pictured in Figure 4a, as a solution to the counter torque problems experienced with traditional helicopters. The multirotor platform has recently grown in popularity, an example is the Parrot AR Drone in Figure 4b, due to multirotor vehicles expanding on the usefulness and benefits of standard rotor-craft helicopter platforms with the additional gains of design simplicity, lower costs and smaller frame size. Furthermore, the rise of small and powerful computational hardware has solved previous problems of high pilot workload when dealing with the multirotor's complex dynamics, outlined in Chapter 5. 


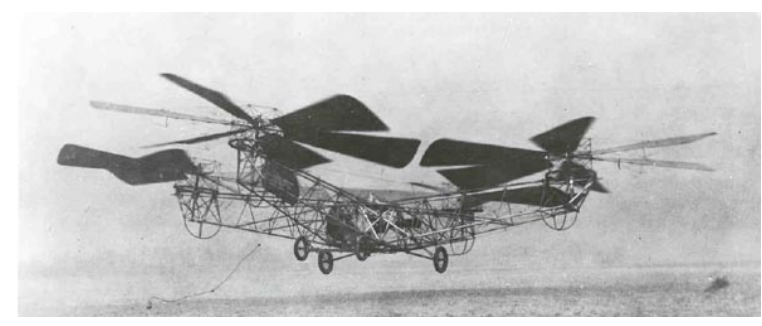

(a) de Bothezat Helicopter [27]

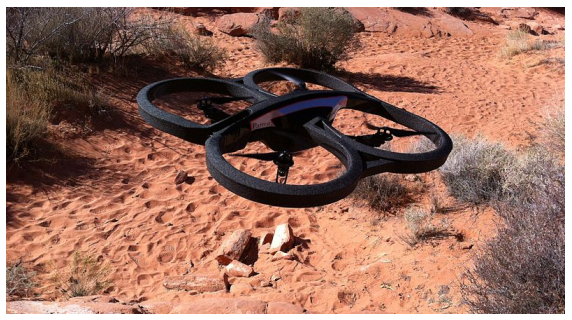

(b) Parrot AR Drone [28]

Figure 4: Evolution of multirotor aircraft

This has lead to the multirotor becoming extremely popular, most notably for aerial photography applications, and the focus of much research to further expand its applications. In general, a multirotor can be simplified to a flying vehicle with a rotating constant pitch propeller propulsion system at the end of a set of symmetric arms. Therefore, the lift of the quadrotor is produced solely by the combined thrust of the propellers. While this is less efficient than a comparable helicopter with an actuated blade system, the simplicity of only using a constant pitch results in an economical propulsion system and easier maintenance.

While multirotors can have any number of motors, typical configurations have three, four, six, or eight arms as shown in Figure 5. The thrust of each individual motor is then used to control the attitude and position of the vehicle. The details of how different motors are used to control the vehicles attitude and position are presented in Section 4.2 .

Similar to any aircraft design, the frame selection is a function of the intended mission 


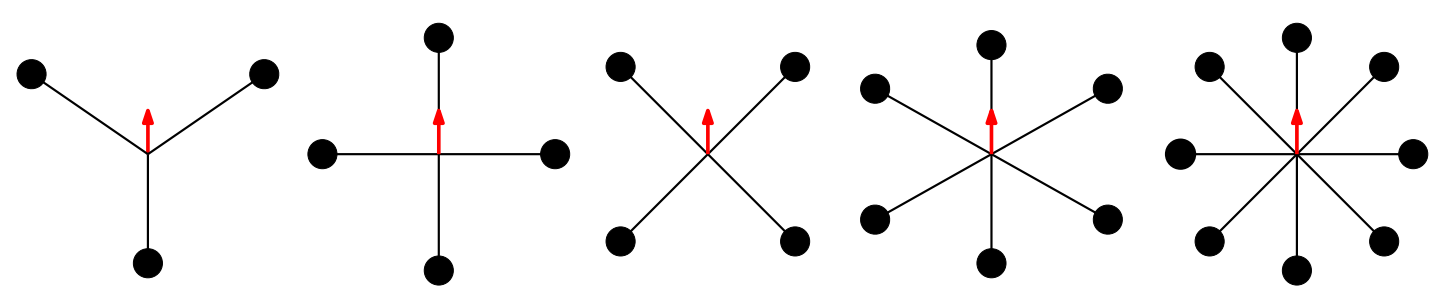

Figure 5: Typical multirotor frames, tri, quad-plus, quad-cross, hex, and octa

profile and desired flight characteristics such as speed, agility, endurance, or reliability. The quadrotor is a popular configuration for both hobbyists and researchers, and while it lacks the power and redundancy of the larger multirotors, it offers manufacturing simplicity and lower frame cost leaving budget for other components and systems.

\subsection{Wind Modeling and Applications}

Intuitively the largest impact on any aerial vehicle's flight performance is the surrounding environment, most notable the resulting forces due to wind and gusts. While larger and heavier 'full sized' aircraft are not as sensitive to external wind effects, in comparison to a SUA such a quadrotor, wind must still be taken into consideration for flight performance and structural loading. In practice this is achieved using continuous gust models to represent atmospheric turbulence [29], where the two most common are the Dryden and von Karman models. These models describe the velocity components using power spectral density functions [29] while assuming a stationary Gaussian process. Both models are functions of a turbulent length scale and turbulence intensity scale dependent on altitude due to varying global effects such as wind shear and temperature gradients. While the models utilize many simplifications for clean air turbulence, they are computationally 
simple to implement and produce satisfactory turbulent results for general flight dynamics study. For this reason the Dryden model has become a FAA standard when designing aircraft. While a continuous gust model can be used for studying appropriate safety envelopes, as outlined in Richardson et al. [30], and has even been used in the pursuit of developing UAV control schemes to incorporate general external turbulent disturbances by Waslander et al. [19] or Langelaan et al. [31], the simplifications of the continuous gust models become inappropriate inside the urban canopy layer.

There are four important length scales used in classifying flow in urban environments, regional (100-200 km), city $(10-20 \mathrm{~km})$, neighborhood $(1-2 \mathrm{~km})$ and street level (100-200 m) defined in Britter et al. [32]. At the regional and city scales the flow around individual buildings is averaged out and the structure's drag results in flow similar to that over a rough surface resulting in an atmospheric boundary layer (ABL) [33]. Therefore the ABL is comprised of two main sections, the outer and surface layers, where the latter is further subdivided based on the resulting flow-surface interactions, as illustrated in Figure 6 .

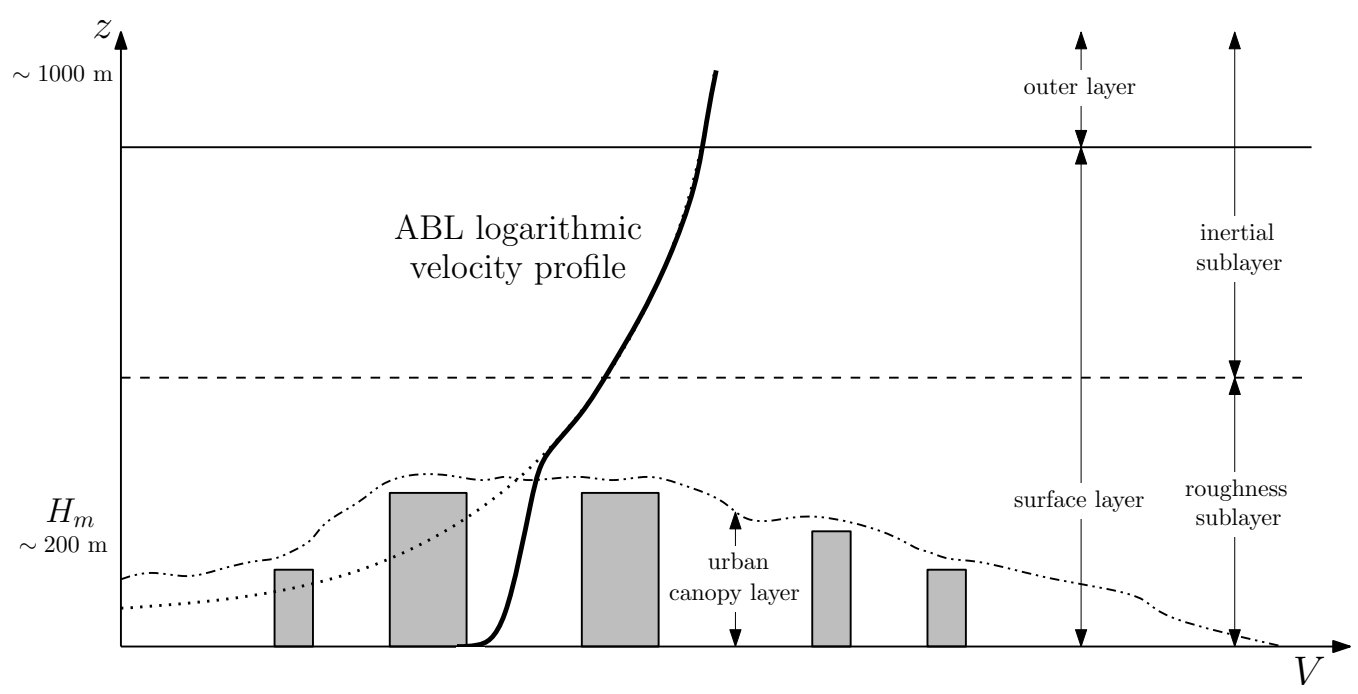

Figure 6: Spatially averaged mean atmospheric boundary layer velocity profile near an urban area. Adapted from Britter [32] and Bottema [34]. 
The flow in the outer freestream is ideally generalized as geostrophic, which is a balance of the pressure and Coriolis forces from Earth's rotation [35], and where the continuous gust models are most applicable. The surface shear stress produces a friction velocity $u_{*}$, a scaling factor for the Monin-Obukhov similarity theory, and in combination with a displacement height $d$, produces a logarithmic profile for the wind velocity in the surface layer.

$$
V(z)=\frac{u_{*}}{\kappa} \ln \left[\frac{z-d}{z_{0}}\right]
$$

Where $\kappa$ is the von Karman constant and $z_{0}$ is the effective surface roughness factor. The factor can take values such as $0.0005 \mathrm{~m}$ for "smooth" terrain with vegetation like beaches or open country, or $2.0 \mathrm{~m}$ for "chaotic" terrain like city centers with a mix of low and high-rise buildings [36]. However, this velocity formation is only applicable for altitudes greater than $2 H_{m}$ were $H_{m}$ is the mean building height.

The inner surface layers and the velocity profile within are highly dependent on the surface roughness and geometry specific. It is within this layer the desired urban flight environment of SUA and MUA exists. The altitude of $H_{m}$, see Figure 6, for typical urban environment is approximately 100 to $200 \mathrm{~m}$, which falls into the street level scale. At the street level scale the bottom most portion of the roughness sublayer contains the urban canopy layer where the flow is directly affected by the size and orientation of local objects such as buildings [32]. Therefore the random noise models of Dryden and von Karman are replaced and enhanced with computational fluid dynamics (CFD) modeling methods. While various studies have been performed in regards to applying wind on UAVs, few use a full transient 
modeled velocity field and use simplifications to save the CFD computational time. For example Escareno et al. [37] applies a 2D approximation, while Guerrero et al. [38], Chen et al. [39], Kothari et al. [17], and Ferrier et al. [40] applied to a Fire Scout MQ-8, use constant prescribed wind values.

The three main methods of CFD consist of Reynolds-averaged Navier-Stokes (RANS), large-eddy simulation (LES) and direct numerical simulation (DNS), where each successive modeling technique yields more realistic approximations. As detailed in Chapter 2, RANS utilizes time averaging simplifications, LES resolves all but the small isotropic motions, and DNS directly simulates all turbulent scales. However, the increased modeling realism comes at the cost of required computation power and time, therefore RANS and LES are commonly used to economically model velocity fields.

Both RANS and LES CFD methods are heavily used with respect to simulating the flow in urban environments, where the latter is becoming evermore accessible and feasible with advent of low cost computing power. Notably several studies have been performed comparing RANS and LES in the context of scalar concentration dispersion in the form of pollutant dissemination, such as Gousseau et al. [41], Walton et al. [42], and Salim et al. [43]. LES has also been used in the application of wind loading on buildings studied by Tamura T. [44] and pedestrian comfort level in urban environments by He et al. [45]. 
CFD methods have also been used with regards to manned aerial vehicles on naval vessels, as the aft landing pad experiences wind conditions similar to that behind urban structures. Bogstad et al. [46] use an Euler based CFD ship-airwake database for use in a flight simulator to train helicopter pilots for landing and takeoff. The shortcomings of the time-averaged flow and its effect on the results are briefly outlined in Zan S. [47]. Forrest et al. [48] use unsteady RANS to model the airwakes around a Type 23 Royal Navy frigate for a flight simulator to test operating limits of helicopter pilots. Crozon et al. [49] studied both steady and unsteady RANS flow around a Canadian Patrol Frigate and its effect on helicopter rotors.

The work of van Muijden et al. [50] compares airwakes around a Landing Platform Dock using both RANS and RANS-LES hybrid CFD methods. In comparison to experimental data van Muijden et al. [50] concludes that the hybrid method has better potential for capturing a flow's physical content suggesting the validity of incorporating LES methods when solving for this type of flow fields.

The previous work of Galway et al. [13-15] has a database of wake fields using RANS CFD methods for testing the flight performance of a fixed wing Aerosonde UAV [15] and Yamaha R-50 rotor-craft [14]. Based on growing popularity and accessibility of LES methods, and its limited application to SUA such as quadrotors in urban environments, this work aims to determine if the additional computational cost of LES is justified in comparison to RANS. 


\subsection{Urban Environment}

The majority of North American cities are a collection of rectangular prisms that act as a bluff body in the presence of wind. This has two benefits; knowledge of expected flow structures from research in general bluff body aerodynamics, and the urban domain can be broken down into simpler geometric blocks. The flow features, specifically the turbulent wake region and multi-building interactions around general bluff bodies, are well documented in experimental studies such as Martinuzzi et al. [51], Dutta et al. [52], and Elshorbagy et al. [53]. While it is desirable and simpler to simulate an entire urban domain on the city or neighbourhood scale, as some studies have done such as Houda et al. [54], or Labovsky et al. [55] for pollutant dispersion or Orr et al. [16] for large fixed wing UAVs, this would be computationally expensive to resolve flow scales on the quadrotor size. Since the desired fixed-wing and quadrotor platforms have scales two orders of magnitude lower than the street level, the previous work of Galway et al. [13-15] is used to reduce the size and computational cost of resolving the wind velocities in a typical North American city. This consists of breaking the urban environment into simplified geometric building blocks for the CFD simulation and then arranging different combinations of these blocks to replicate a desired urban environment. The two most basic building blocks are a single building in isolation and an urban canyon, illustrated in Figures 7a and $7 \mathrm{~b}$ respectively.

Each building block is parametrized with a set of defining characteristics. A single

building is parametrized with the ratio of the building length to width $\left(R_{\perp} / R_{\|}\right)$, the 


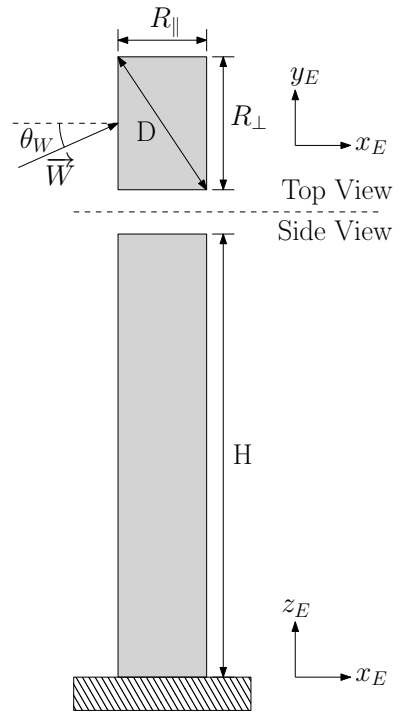

(a) Single building

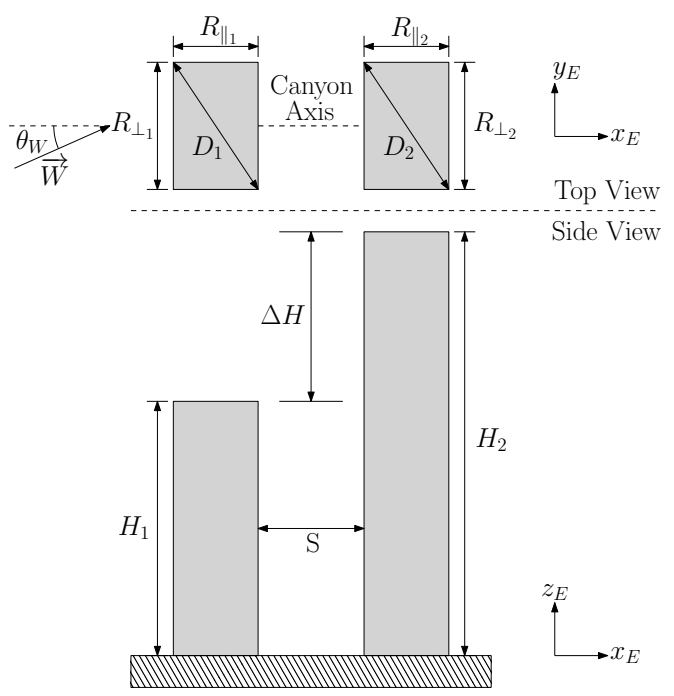

(b) Urban canyon

Figure 7: Primary building block parameters

Reynolds number $\left(R e_{D}\right)$, and the wind incidence angle $\left(\theta_{W}\right)$. The Reynolds number is based on the freestream wind velocity $V_{\infty}$, the characteristic length $D=\sqrt{R_{\perp}+R_{\|}}$, and the kinematic viscosity $\nu$ such that,

$$
\operatorname{Re}_{D}=\frac{V_{\infty} D}{\nu}
$$

The wind vector $\vec{W}$ can be offset at a wind incidence angle $\theta_{W}$ representing the building's orientation with respect to the freestream wind. An urban canyon geometry is formed when single buildings are close enough to generate flow interactions not otherwise found with a single building in isolation. One such interaction is the generation of a turbulent canyon vortex contained within the canyon street length. The urban canyon is parametrized with a set of characteristic lengths for each building with the addition of the street separation distance $\mathrm{S}$, and a height differential $\Delta H$. The $\Delta H$ shown in Figure $7 \mathrm{~b}$ is negative by convention and 
classified as a step up notch.

Therefore by varying the non-dimensional parameters and performing multiple CFD simulations, a database of urban flow fields can be generated and combined to build up a suitable urban flight environment. This environment can then be used with a flight simulator to design control algorithms and to test autonomous flight performance.

The previous work of Galway et al. [13-15] generated a database of wake fields using RANS CFD methods and ANSYS CFX for testing a fixed wing Aerosonde UAV [15] and Yamaha R-50 rotor-craft [14]. This study builds on the previous work by generating a subset of the database using LES and OpenFOAM for the application of testing a quadrotor in urban wake fields. For this a single square building geometry is used, with parameters of $R e=7.30 \times 10^{6}, \theta_{W}=0$ and $\left(R_{\perp} / R_{\|}\right)_{w w}=1$.

As outlined in Sections 2.3 and 2.4 LES is computationally more expensive than RANS due to the additional resolved turbulent motions, however, it is believed resolving these motions are important in the pursuit of designing and testing quadrotor control algorithms for urban flight. Therefore this work compares the autonomous flight performance of a quadrotor with various background wind conditions such as constant wind, RANS generated wind and LES generated wind to determine if the additional computational cost of LES is required in the development of appropriate autonomous flight control methods. 


\section{Chapter 2}

\section{Methodology}

\subsection{OpenFOAM}

The urban wake fields are generated using the Open Source Field Operation and Manipulation (OpenFOAM) CFD package [56]. While the workflow is similar to a commercial product, OpenFOAM is a collection of $\mathrm{C}++$ libraries which provide various solver and utility applications [56]. As illustrated in Figure 8, the solvers of OpenFOAM are able to address many different continuum problems; from incompressible fluid flow to modeling an electric field. Similarly OpenFOAM comes with many useful utilities for problem setup and post-processing data manipulation. The specific utilities employed to generate the wake fields are outlined on the far

right of Figure 8 such as the snappyHexMesh meshing utility or the PISO solving algorithm. Version 2.2.x of OpenFOAM is used in combination with the open source 3D visualization application Paraview version 3.12. Both applications run on a workstation with a 64-bit OpenSUSE 12.4 Linux OS, dual Intel Xenon E5-2687W octo-core CPUs and 64 GB of RAM. 


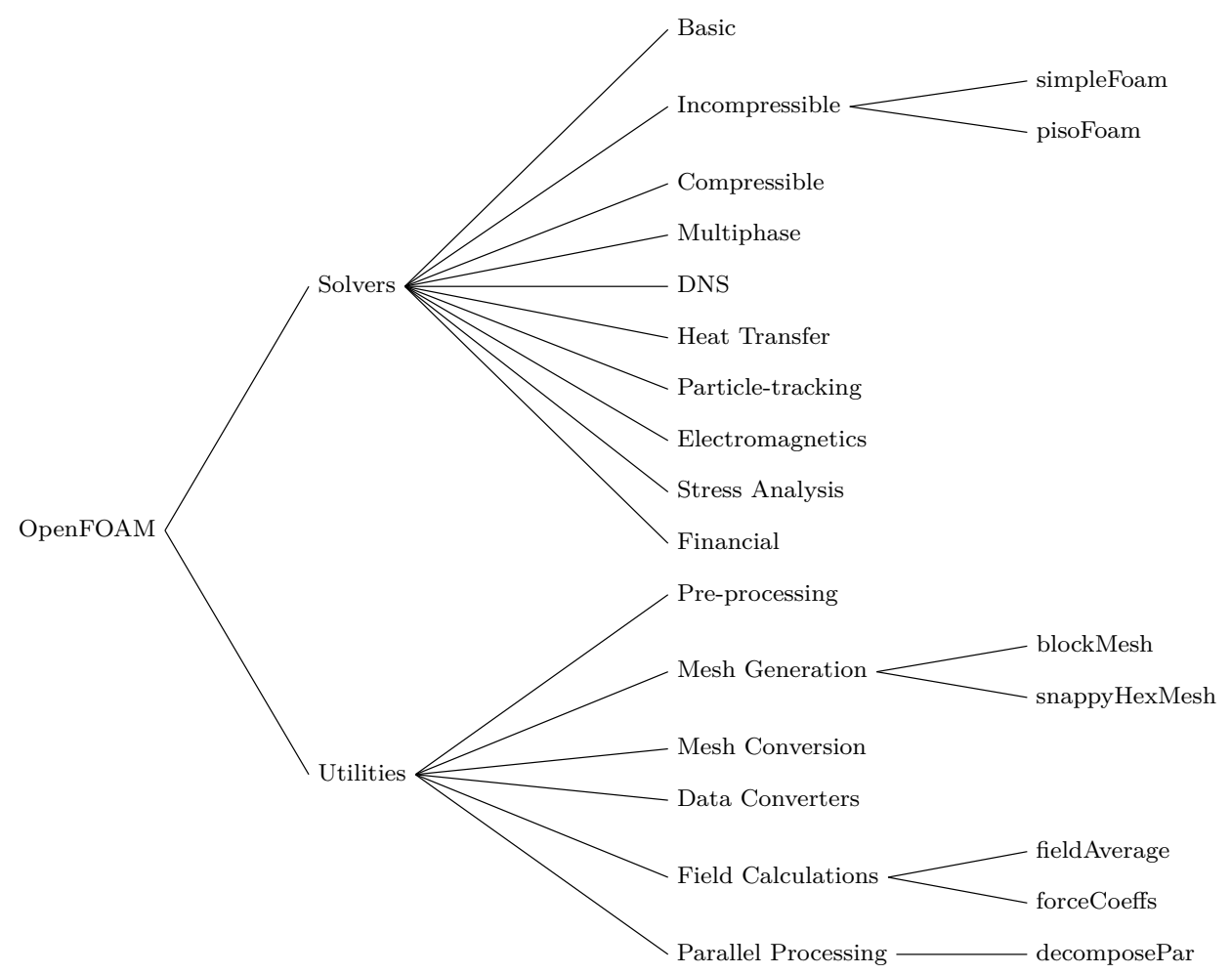

Figure 8: Sample of used OpenFOAM solver and utilities libraries.

OpenFOAM is used to regenerate a subset of the wake fields based on previous work using RANS modeling as well as performing the proposed LES methods. OpenFOAM utilizes the finite volume approach for spatial discretization of the governing equations. These equations are solved using one of two solving algorithms, the SemiImplicit Method for Pressure-Linked Equations (SIMPLE) or the Pressure Implicit with Splitting of Operators (PISO) method [57]. The PISO algorithm iteratively calculates the pressure-velocity coupling using a predictor and corrector approach as illustrated in Figure 9. The PISO algorithm is an extension of the SIMPLE method where no under-relaxation is applied and two momentum corrector steps are performed. 


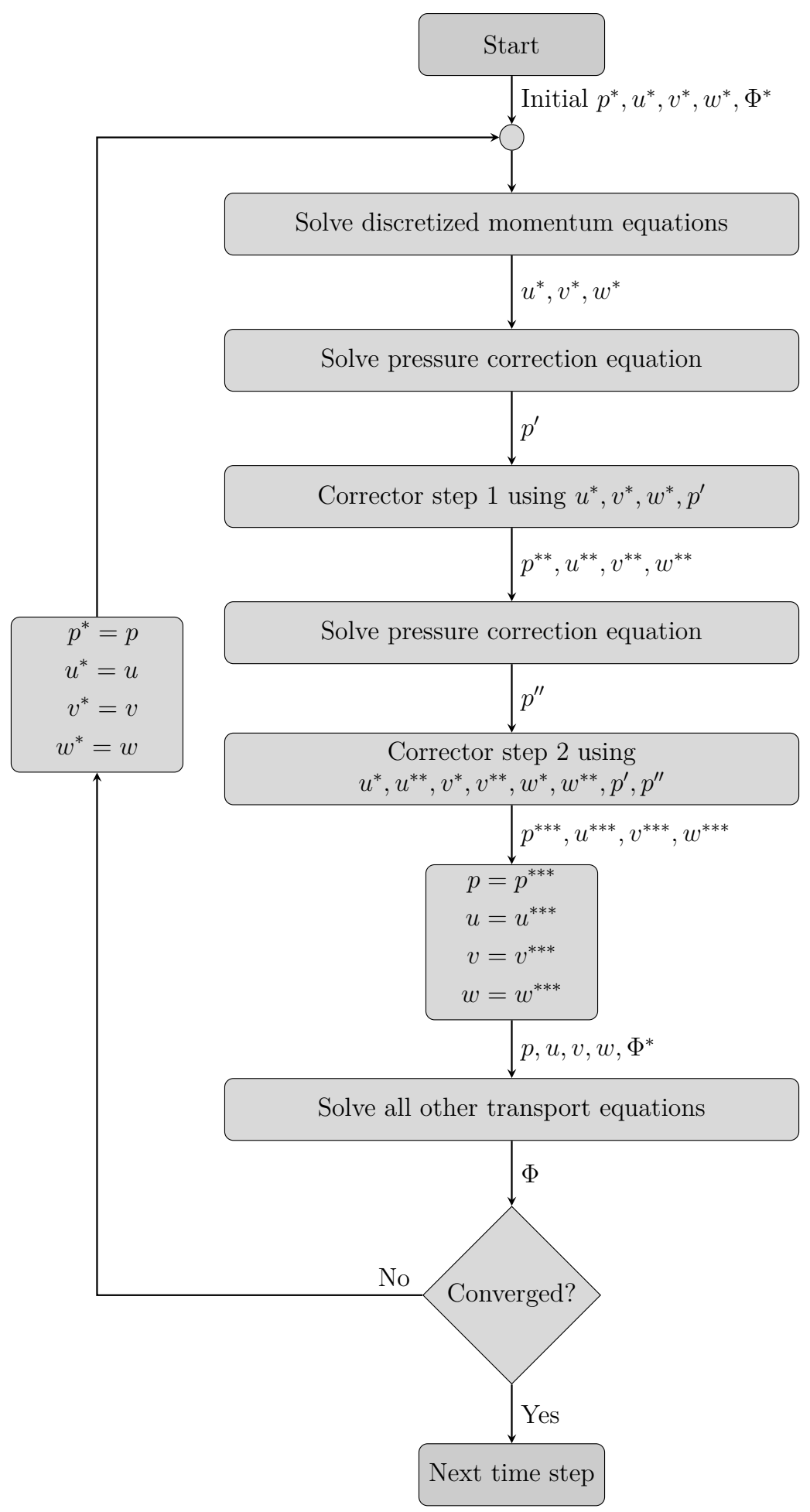

Figure 9: Two corrector step PISO algorithm 
The SIMPLE algorithm is used when generating a turbulent velocity field with a steady RANS simulation in advance of the LES to decrease the start up time between the initial uniform conditions and the generation of turbulent structures. The RANS simulation uses the PISO algorithm to transiently solve the governing equations outlined in Subsection 2.3 and the timestep is specified such that the Courant-Friedrichs-Lewy (CFL) number is 1.0 in the smallest grid cells. The LES is also solved with the transient PISO algorithm but the timestep is specified such that the CFL number is maintained within a range of 0.4-0.6 in the smallest grid cells. This range of CFL is used to ensure stability as well as allowing for the appropriate turbulent timescales to be resolved [58].

Since OpenFOAM is just a collection of libraries and utilities there are several ways to interact with them. While there are options for graphical user interfaces (GUIs), such as HELYX ${ }^{\mathrm{OS}}$, OpenFOAM has the ability to run under Linux bash shell script control or through Python scripts with pyFoam. To aid the setup, execution and proceeding of additional simulations to generate a LES based wind database the standardized file structure used is shown in Figure 10. After setting the simulation specific details in the various Setup files, such as the freestream wind velocity, geometry names, meshing densities, and parallel processors, the Allrun script is called to perform the steady RANS simulation, map the results and run the LES. The additional collection of scrips in the PostProcessing sub-folder allow for LES wake data extraction, trimming and uploading. The details of the post processing scripts and the file structure are outlined in Appendix A. 
Standard Working Directory/

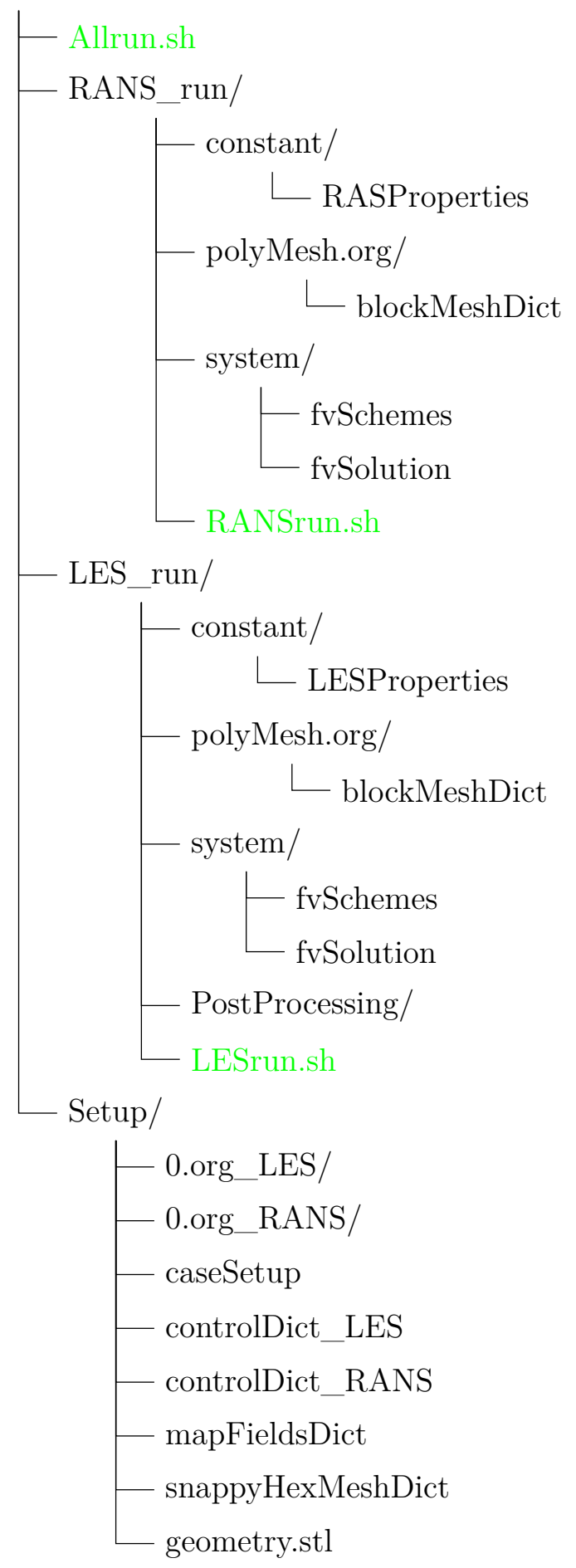

Figure 10: Outline of the standard LES working directory 


\subsection{Urban Wake Field CFD Modeling}

The idea and complication of turbulence in fluid flow has existed since the work of Leonardo da Vinci and modernized by the classic pipe experiment by Osborne Reynolds in 1883 [59]. Reynolds also proposed and popularized a single dimensionless parameter, the Reynolds Number, which describes the flow behavior as a ratio of inertial to viscous forces. While previously introduced for application to the urban environment the general definition takes the form,

$$
R e=\frac{V L}{\nu}
$$

Over the ensuing decades, the importance of understanding and subsequently the desire to model turbulent flows forced much advancement in the subject area. The notion of turbulent transition was expanded with the concepts such as turbulent length and time scales and the energy cascade. For the sake of brevity the development history of simulating turbulent flow in terms of CFD is not presented [60], rather only the resulting methods and models.

The difficulty arises when the nonlinear differential equations of fluid motion, the Navier-Stokes equations, are further complicated by the addition of terms from the viscous-turbulence relationship. The methods used to numerically solve turbulent flow fall into one of three categories; Reynolds-averaged Navier-Stokes (RANS), large-eddy simulation (LES) and direct numerical simulation (DNS). As previously mentioned this work expands on previous RANS based methods with LES and for this reason the following sections will only outline their characteristics and equations. 


\subsection{Reynolds-Averaged Navier-Stokes Method}

One method to reduce the extensive computational requirements to resolve all of the spatial and temporal scales in turbulent flow is to apply Reynolds decomposition. The basis of the decomposition is time averaging of the flow properties defined as,

$$
\bar{\Phi}\left(x_{i}\right) \equiv \frac{1}{T} \int_{0}^{T} \Phi\left(x_{i}, t\right) d t
$$

where the domain flow quantities $\Phi\left(x_{i}, t\right)$, are broken into mean $\bar{\Phi}\left(x_{i}\right)$ and a fluctuation components $\Phi^{\prime}\left(x_{i}, t\right)$ such that,

$$
\Phi\left(x_{i}, t\right)=\bar{\Phi}\left(x_{i}\right)+\Phi^{\prime}\left(x_{i}, t\right)
$$

and illustrated by,

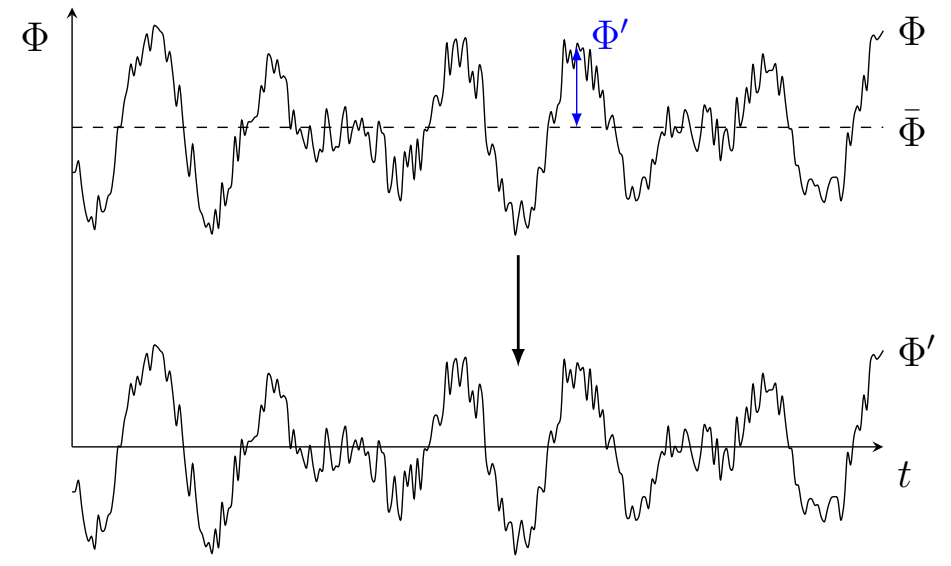

Figure 11: Reynolds decomposition of $\Phi$ over time

The time averaging and decomposition give rise to Reynolds operators, linear algebraic operators on the governing functions. From the definitions of the time averaging in Equation (4) and the fluctuation component in Figure 11, the two most important Reynolds decomposition properties arise such that, 


$$
\begin{aligned}
\bar{\Phi} & =\bar{\Phi} \\
\overline{\Phi^{\prime}} & =0
\end{aligned}
$$

Combining these with the incompressible continuity and momentum equations produces,

$$
\begin{aligned}
& \frac{\partial U_{i}}{\partial x_{i}}=0 \\
& \frac{\partial U_{i}}{\partial t}+U_{j} \frac{\partial U_{i}}{\partial x_{j}}=-\frac{1}{\rho} \frac{\partial P}{\partial x_{i}}+\nu \frac{\partial^{2} U_{i}}{\partial x_{j} \partial x_{j}}
\end{aligned}
$$

where $U$ is the total velocity, $P$ is the pressure, and $\nu$ is the kinematic viscosity. Substituting the Reynolds decomposition and simplifying results in the incompressible RANS continuity equation for the mean flow,

$$
\begin{aligned}
0 & =\frac{\partial U_{i}}{\partial x_{i}} \\
0 & =\frac{\partial\left(\bar{u}_{i}+u_{i}^{\prime}\right)}{\partial x_{i}} \\
0 & =\frac{\partial \bar{u}_{i}}{\partial x_{i}}+\frac{\partial u_{i}^{\prime}}{\partial x_{i}} \\
0 & =\frac{\bar{u}_{i}}{\partial x_{i}}+\frac{\overline{u_{i}^{\prime}}}{\partial x_{i}} \\
0 & =\frac{\partial \bar{u}_{i}}{\partial x_{i}}
\end{aligned}
$$

To derive the RANS momentum equations, the Reynolds decomposition is applied to both the velocity and pressure quantities in Equation (8),

$$
\underbrace{\frac{\partial\left(\bar{u}_{i}+u_{i}^{\prime}\right)}{\partial t}}_{I}+\underbrace{\left(\bar{u}_{j}+u_{j}^{\prime}\right) \frac{\partial\left(\bar{u}_{i}+u_{i}^{\prime}\right)}{\partial x_{j}}}_{I I}=\underbrace{-\frac{1}{\rho} \frac{\left(\bar{p}+p^{\prime}\right)}{\partial x_{i}}}_{I I I}+\underbrace{\nu \frac{\partial^{2}\left(\bar{u}_{i}+u_{i}^{\prime}\right)}{\partial x_{j} \partial x_{j}}}_{I V}
$$


The linearity of the temporal discretization $I$, pressure $I I I$ and viscous stress terms $I V$ allow simple application of the Reynolds operators and time averaging following the same procedure as the continuity equation. However, application of the RANS averaging on the convection acceleration term, $I I$, requires additional work due to the products arising from the multiplication,

$$
\begin{aligned}
& \left(\bar{u}_{j}+u_{j}^{\prime}\right) \frac{\partial\left(\bar{u}_{i}+u_{i}^{\prime}\right)}{\partial x_{j}} \\
& \bar{u}_{j} \frac{\partial \bar{u}_{i}}{\partial x_{j}}+\bar{u}_{j} \frac{\partial u_{i}^{\prime}}{\partial x_{j}}+u_{j}^{\prime} \frac{\partial \bar{u}_{i}}{\partial x_{j}}+\frac{\partial u_{i}^{\prime} \frac{\partial x_{j}}{\partial x_{j}}}{\bar{u}_{j} \frac{\partial \bar{u}_{i}}{\partial x_{j}}}+\underbrace{\bar{u}_{j} \frac{\partial u_{i}^{\prime}}{\partial x_{j}}}_{I I^{\prime}}+\underbrace{u_{j}^{\prime} \frac{\partial \bar{u}_{i}}{\partial x_{j}}}_{I I I^{\prime}}+\underbrace{\frac{\partial u_{i}^{\prime}}{\partial x_{j}}}_{I V^{\prime}}
\end{aligned}
$$

Applying the definition of time averaging, Equation (4), to the second and third terms, $I I^{\prime}$ and $I I I^{\prime}$, reduces them to zero following,

$$
\begin{aligned}
\overline{\bar{u}_{j} \frac{\partial u_{i}^{\prime}}{\partial x_{j}}} & =\frac{1}{T} \int_{0}^{T}\left(\bar{u}_{j} \frac{\partial u_{i}^{\prime}}{\partial x_{j}}\right) d t \\
& =\bar{u}_{j} \frac{\partial}{\partial x_{j}}\left(\frac{1}{T} \int_{0}^{T}\left(u_{i}^{\prime}\right) d t\right) \\
& =\bar{u}_{j} \frac{\partial \overline{u_{i}^{\prime}}}{\partial x_{j}} \\
& =0
\end{aligned}
$$

Therefore collecting the averaged terms and simplifying Equation (10),

$$
\frac{\partial \bar{u}_{i}}{\partial t}+\underbrace{\overline{\bar{u}_{j} \frac{\partial \bar{u}_{i}}{\partial x_{i}}}}_{I^{\prime \prime}}+\underbrace{\overline{u_{j}^{\prime} \frac{\partial u_{i}^{\prime}}{\partial x_{i}}}}_{I I^{\prime \prime}}=-\frac{1}{\rho} \frac{\partial \bar{p}}{\partial x_{i}}+\nu \frac{\partial^{2} \bar{u}_{i}}{\partial x_{j} \partial x_{j}}
$$


A substitution is made for the remaining nonlinear terms, $I^{\prime \prime}$ and $I I^{\prime \prime}$, culminating in the time-averaged momentum equations,

$$
\begin{aligned}
\frac{\partial \bar{u}_{i}}{\partial t}+\frac{\overline{\partial \bar{u}_{i} \bar{u}_{j}}}{\partial x_{j}}+\frac{\overline{\partial u_{i}^{\prime} u_{j}^{\prime}}}{\partial x_{j}} & =-\frac{1}{\rho} \frac{\partial \bar{p}}{\partial x_{i}}+\nu \frac{\partial^{2} \bar{u}_{i}}{\partial x_{j} \partial x_{j}} \\
\frac{\partial \bar{u}_{i}}{\partial t}+\frac{\partial \bar{u}_{i} \bar{u}_{j}}{\partial x_{j}}+\frac{\partial \overline{u_{i}^{\prime} u_{j}^{\prime}}}{\partial x_{j}} & =-\frac{1}{\rho} \frac{\partial \bar{p}}{\partial x_{i}}+\nu \frac{\partial^{2} \bar{u}_{i}}{\partial x_{j} \partial x_{j}} \\
\frac{\partial \bar{u}_{i}}{\partial t}+\frac{\partial}{\partial x_{j}}\left(\bar{u}_{i} \bar{u}_{j}\right) & =-\frac{1}{\rho} \frac{\partial \bar{p}}{\partial x_{i}}+\nu \frac{\partial^{2} \bar{u}_{i}}{\partial x_{j} \partial x_{j}}-\frac{\partial}{\partial x_{j}} \overline{u_{i}^{\prime} u_{j}^{\prime}}
\end{aligned}
$$

The $\overline{u_{i}^{\prime} u_{j}^{\prime}}$ term is a product of the non-linear convection term and represents the convective momentum transfer from turbulent eddies [57]. This quantity is called the Reynolds stress tensor which cannot be related to the unknown mean velocity and pressure fields and therefore results in the 'turbulent closure problem' [60]. Therefore to predict the Reynolds stresses based on the mean flow and close the system of equations, turbulence models are employed.

All current models use one of two methods to ascertain the unknown Reynolds stresses, the eddy viscosity relation or the Reynolds stress model (RSM). The most common models use eddy viscosity which is loosely based on Newton's law of viscosity where the shear stress is proportional to the strain rate,

$$
\tau=\mu \frac{\partial u}{\partial y}
$$

Where $\mu$ is the fluid's dynamic viscosity. The Boussinesq hypothesis expands this expression for a turbulent flow case to relate Reynolds stresses to the mean rates of viscous deformation, 


$$
-\overline{u_{i}^{\prime} u_{j}^{\prime}}=\nu_{t}\left(\frac{\partial \bar{u}_{i}}{\partial x_{j}}+\frac{\partial \bar{u}_{j}}{\partial x_{i}}\right)-\frac{2}{3} k \delta_{i j}
$$

Where $\nu_{t}$ is the turbulent eddy viscosity, $k$ is the specific turbulent kinetic energy and $\delta_{i j}$ is the Kronecker delta. The contribution of this additional term ensures a correct relation for the normal Reynolds stress components [57]. While this approximation aids in the closure problem, it introduces an additional unknown and complex variable $\nu_{t}$. Therefore the principal goal of any turbulence model is to calculate $\nu_{t}$ either with simple relationships, such as Prandtl's mixing length model or through additional transport equations, such as the $k-\varepsilon$ or $k-\omega$ SST models.

The two equation $k-\varepsilon$ model is briefly outlined as it is used to generate an urban wake field based on previous urban wind generation work of Galway et al. [13-15]. The $k-\varepsilon$ model introduces a transport equation for the turbulent kinetic energy $k$ and the specific turbulent kinetic energy dissipation rate $\varepsilon$ to provide velocity and length scales to find the unknown turbulent eddy viscosity. The specific turbulent kinetic energy is defined as,

$$
k=\frac{1}{2} \overline{u_{i} u_{i}}=\frac{1}{2}\left(\overline{u^{\prime 2}}+\overline{v^{\prime 2}}+\overline{w^{\prime 2}}\right)
$$

and the specific turbulent kinetic energy dissipation rate is defined as,

$$
\varepsilon=2 \nu \overline{s_{i j}^{\prime} s_{i j}^{\prime}}
$$

where $s_{i j}^{\prime}$ is the fluctuating strain-rate tensor,

$$
s_{i j}^{\prime}=\frac{1}{2}\left(\frac{\partial u_{i}^{\prime}}{\partial x_{j}}+\frac{\partial u_{j}^{\prime}}{\partial x_{i}}\right)
$$

Using these definitions, the RANS momentum Equation (14c), and considerable manipulation, produces the additional transport equations shown from Tennekes 
and Lumley [61] or Launder and Spalding [62],

$$
\begin{array}{r}
\frac{\partial k}{\partial t}+\bar{u}_{j} \frac{\partial k}{\partial x_{j}}=-\overline{u_{i}^{\prime} u_{j}^{\prime}} \frac{\partial \bar{u}_{i}}{\partial x_{j}}-\varepsilon+\frac{\partial}{\partial x_{j}}\left[\left(\nu+\nu_{t} / \sigma_{k}\right) \frac{\partial k}{\partial x_{j}}\right] \\
\frac{\partial \varepsilon}{\partial t}+\bar{u}_{j} \frac{\partial \varepsilon}{\partial x_{j}}=-C_{1} \frac{\varepsilon}{k} \overline{u_{i}^{\prime} u_{j}^{\prime}} \frac{\partial \bar{u}_{i}}{\partial x_{j}}-C_{2} \frac{\varepsilon^{2}}{k}+\frac{\partial}{\partial x_{j}}\left[\left(\nu+\nu_{t} / \sigma_{\varepsilon}\right) \frac{\partial \varepsilon}{\partial x_{j}}\right]
\end{array}
$$

Finally the system of equations are closed by relating these transport equations to the eddy viscosity using dimensional analysis resulting in,

$$
\nu_{t}=C_{\nu} \frac{k^{2}}{\varepsilon}
$$

The final requirement in closing the model is addressing the five unknown constants. The standard OpenFOAM $k-\varepsilon$ constants are used [56], as shown below, and are the result of comprehensive data fittings from experimental results,

$$
C_{\nu}=0.09 \quad C_{1}=1.44 \quad C_{2}=1.92 \quad \sigma_{\varepsilon}=1.30 \quad \sigma_{k}=1.00
$$

\subsection{Large-Eddy Simulation Method}

While RANS based methods are the most common in the engineering industry, the available turbulent models presume universal behavior of the various turbulent scales. However, only the small turbulent eddies are more universal and isotropic in nature in comparison to the large energy containing eddies. While the influence of the small eddies on the flow is required, resolving down to the Kolmogorov length scale is computationally expensive in terms of spacial and temporal resolution. LES was created to directly resolve the transient and geometry dependent motions while the energy draining effects of the small eddies on the resolved flow is modeled to 
save computational cost [63]. Because the large-scale unsteady motions are directly resolved with LES, it can be expected to be more accurate than RANS methods when modeling flow with large scale unsteadiness, such as flow over bluff bodies like urban structures [63].

At its core LES is a low-pass filter, applied to attenuate high frequency turbulent motions while leaving the low frequency motions unaltered. This is performed by applying a spacial filter with a cutoff width to define the resolved and modeled length scales. Figure 12 illustrates the turbulent kinetic energy spectrum as a function of wavenumber and how the filter cutoff width $\left(k_{c}\right)$ separates the resolved and sub-grid-scale (SGS) length scales.

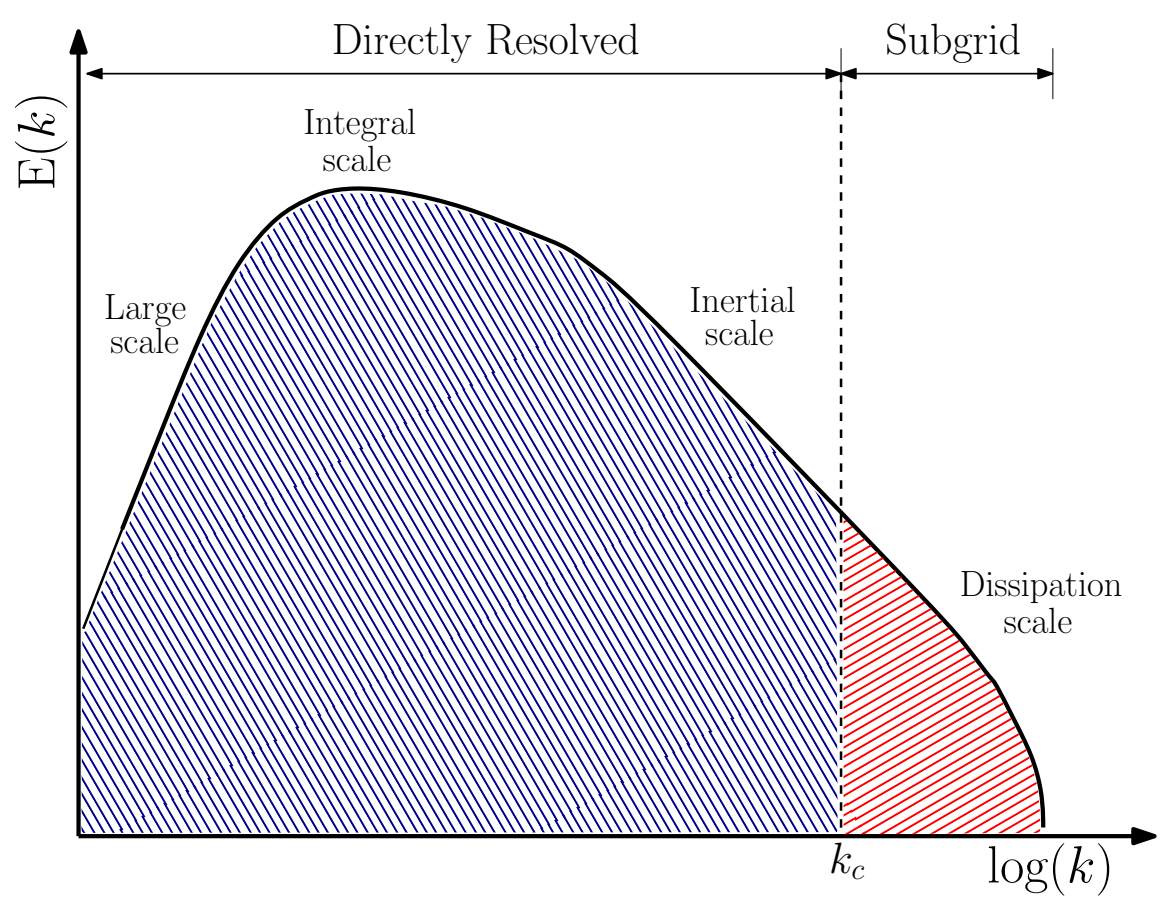

Figure 12: Turbulent kinetic energy spectrum with LES cutoff width 
The definition of the spatial filtering process, as introduced by Leonard [64], takes the form of,

or,

$$
\tilde{\Phi}(x, t)=\int_{V} G(r, x, \Delta) \Phi(x-r, t) \mathrm{d} r
$$

$$
\widetilde{\Phi}=G \cdot \Phi
$$

where $G$ is a filter function, $\Delta$ is the filter width, $\Phi$ is the original unfiltered flow variable, $\tilde{\Phi}$ is the filtered flow variable, $x$ is a global coordinate frame, and $r$ is a cell local axis. The residual field is defined such that,

$$
\Phi(x, t)^{\prime} \equiv \Phi(x, t)-\tilde{\Phi}(x, t)
$$

or,

$$
\Phi^{\prime}=(1-G) \cdot \Phi
$$

where the total flow variables $(\Phi)$ are separated into filtered $(\widetilde{\Phi})$ and subgrid-scale components $\left(\Phi^{\prime}\right)$. While visually similar to the Reynolds decomposition of Equation (5), the filtering does not generally follow the rules of a Reynolds operator, most notably,

$$
\begin{aligned}
\widetilde{\widetilde{\Phi}} & \neq \widetilde{\Phi} \\
\tilde{\Phi}^{\prime} & \neq 0
\end{aligned}
$$

since,

$$
\begin{aligned}
\widetilde{\widetilde{\Phi}} & =G \cdot G \cdot \Phi=G^{2} \cdot \Phi \neq \widetilde{\Phi}=G \cdot \Phi \\
\widetilde{\Phi^{\prime}} & =G \cdot(1-G) \cdot \Phi \neq 0
\end{aligned}
$$


The decomposition of Equation (24) and filter properties of Equations (26a) and (26b) are artistically illustrated in Figure 13. From this it is very clear that; a double filter is not equal to a single filter application $(\widetilde{\widetilde{\Phi}} \neq \widetilde{\Phi})$ and the filtered residual is not zero $\left(\tilde{\Phi}^{\prime} \neq 0\right)$.

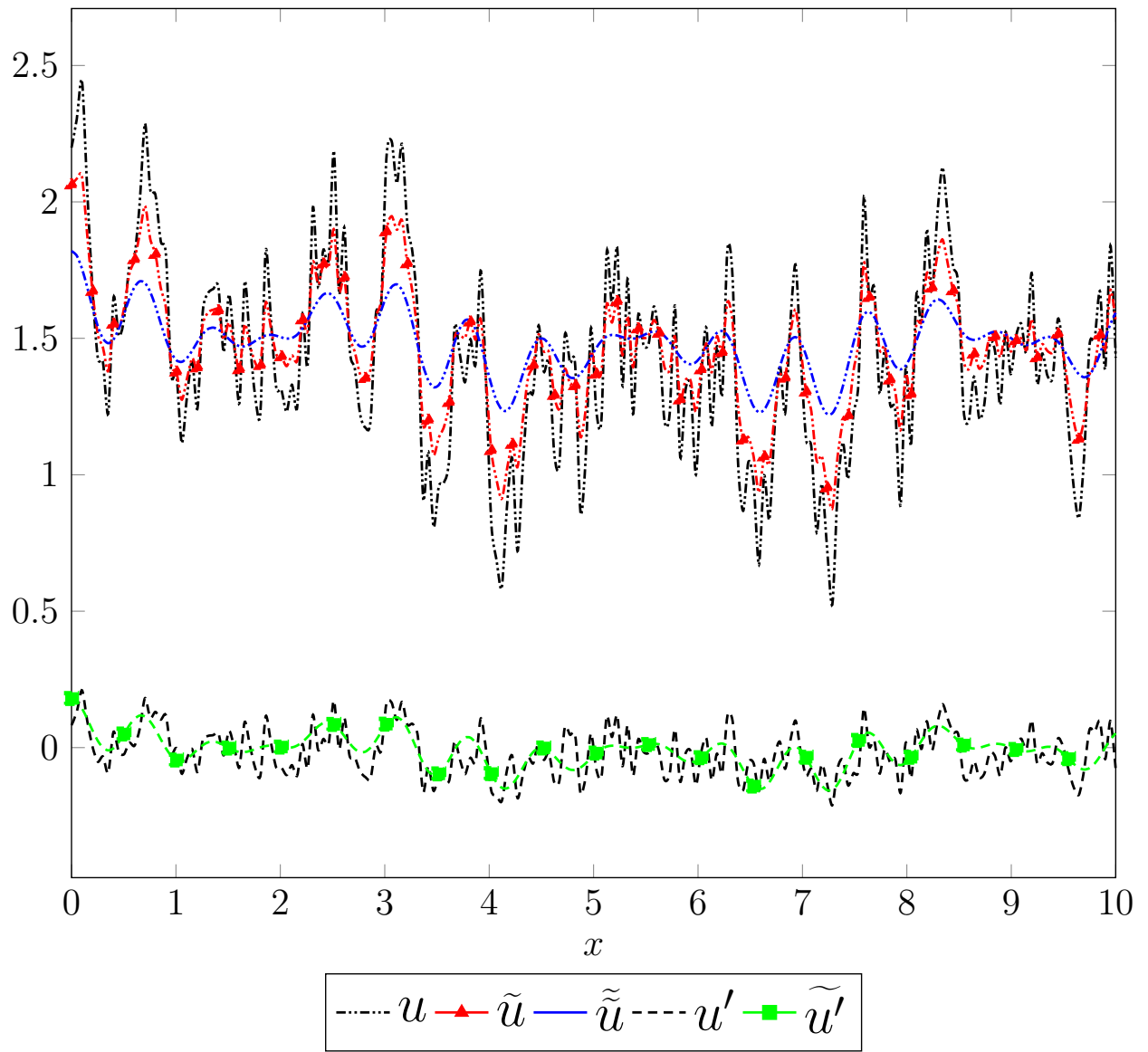

Figure 13: LES decomposition and filter properties. Adapted from Pope [63]. 
The common most forms of the filter function $(G)$ for LES include the box, Gaussian, and sharp spectral respectively defined in one dimension as,

$$
\begin{aligned}
& G(x, \Delta)=\left\{\begin{array}{rr}
1 / \Delta & :|x-r| \leq \Delta / 2 \\
0 & : \text { otherwise }
\end{array}\right. \\
& G(x, \Delta)=\sqrt{\frac{6}{\pi \Delta^{2}}} \exp \left(-\frac{6(x-r)^{2}}{\Delta^{2}}\right) \\
& G(x, \Delta)=\frac{\sin [\pi(x-r) / \Delta]}{\pi(x-r)}
\end{aligned}
$$

These filters are shown in Figure 14, illustrating both the active interval, normalized by $\Delta$, and the filters function's value.

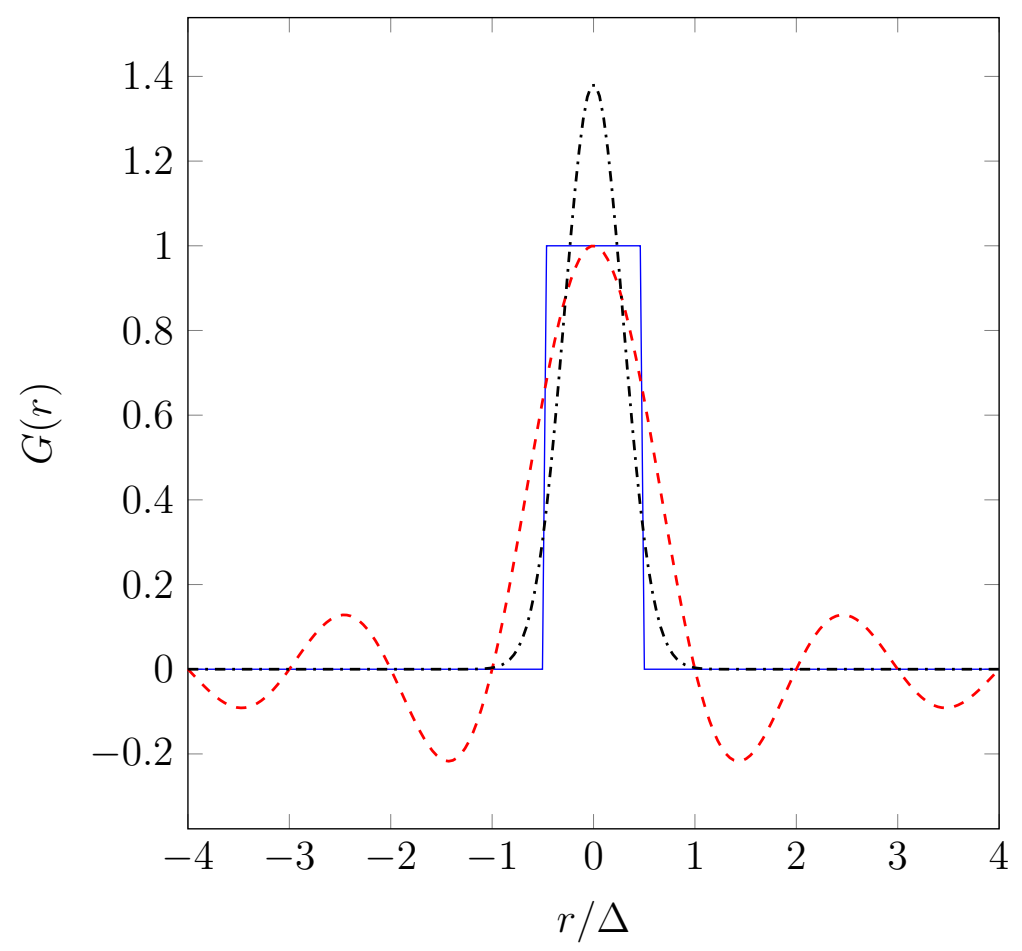

— Box - - Gaussian -.-.. Sharp Spectral

Figure 14: Common LES filter functions. Adapted from Pope [63]. 
The box filter is applied in finite volume implementations of LES, where the Gaussian and spectral cutoff filters are preferred in the research literature [57]. The Gaussian filter has the advantage of being smooth and differentiable [63], while the sharp spectral eliminates all wave numbers above a chosen frequency [57]. However, since the box filter is just an average over the filter interval, and the flow variables are piecewise linear functions of $\mathrm{x}$ for finite volume methods, if the filter width is equal to the grid-spacing, a box filter is simply a local cell spatial average. [58]. In other words, the filter width is indicative of the size of eddies retained (see Figure 12), and can be chosen to be any size. Since finite volume methods only retain a single node value for each cell there is no resolution benefit to specifying a filter width smaller than the grid size [57]. This is classified as implicit LES as the filter width is implicitly determined from the cell size, and the most accepted method of defining the filter width is to use the cube root of the cell volume,

$$
\Delta=\sqrt[3]{\Delta_{x} \Delta_{y} \Delta_{z}}
$$

An example of a box filter application is shown in Figure 15 using the velocity field resolved from a DNS [65]. A box filter is applied with an increasing filter width between Figures 15b and 15c and illustrates how the smaller higher frequency turbulent eddies are averaged and smoothed out (lower values of $k_{c}$ in Fiugre 12).

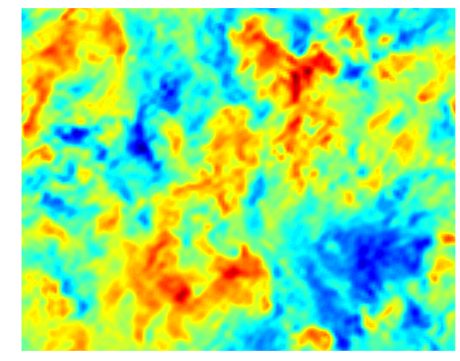

(a) DNS velocity field [66]

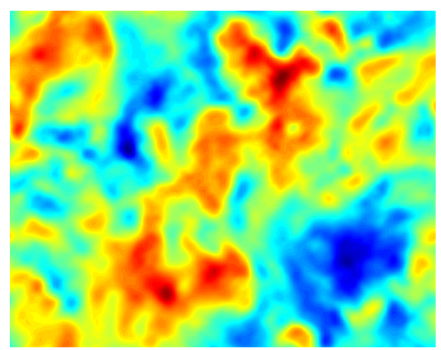

(b) Box filter, $\Delta=L / 32[66]$ (c) Box filter, $\Delta=L / 16$ [66]

Figure 15: Box filter example using DNS velocity field, domain size is $L^{3}$. 
Derivation of the LES governing equations begins with the incompressible continuity and momentum equations, Equations (7) and (8), and application of the filter function,

$$
\frac{\widetilde{\partial u_{i}}}{\partial x_{i}}=0
$$

An important simplification can be made if the filter function is able to commute with temporal and spatial differentiation. It is for this reason the box filter shown in Equation (28a) is only a spatial filter (independent of time) and made locally independent in space through $|x-r|$ (cell based). While this does not hold for all possible filter functions, application of the box filter results in filtered continuity equation,

$$
\frac{\partial \widetilde{u}_{i}}{\partial x_{i}}=0
$$

To derive the LES momentum equations, the LES filtering is applied and the linear terms are simplified in a similar manner to their RANS counterparts,

$$
\frac{\partial \widetilde{u}_{i}}{\partial t}+\frac{\partial}{\partial x_{j}}\left(\widetilde{u_{i} u_{j}}\right)=-\frac{1}{\rho} \frac{\partial \widetilde{p}}{\partial x_{i}}+\nu \frac{\partial^{2} \widetilde{u}_{i}}{\partial x_{j} \partial x_{j}}
$$

Once again the non-linear convection term $\widetilde{u_{i} u_{j}}$ is troublesome to express in terms of known flow variables as the filtered products is different than the product of filtered velocities $\left(\widetilde{u_{i} u_{j}} \neq \widetilde{u}_{i} \widetilde{u}_{j}\right)$. Therefore a modelling approximation is introduced to account for the residual-stress, called the SGS stress tensor,

$$
\tau_{i j}=\widetilde{u_{i} u_{j}}-\tilde{u}_{i} \tilde{u}_{j}
$$

which is not dissimilar to the Reynolds stress tensor in Equation (14c),

$$
\overline{u_{i}^{\prime} u_{j}^{\prime}}=\overline{u_{i} u_{j}}-\bar{u}_{i} \bar{u}_{j}
$$


The tensor has the property such that $\left|\tau_{i j}\right| \rightarrow 0$, as $\Delta \rightarrow 0$, ultimately resulting in a DNS solution in the limit of a small mesh spacing [58]. Substituting Equation (33) into Equation (32) results in the filtered or LES momentum equations,

$$
\frac{\partial \widetilde{u}_{i}}{\partial t}+\frac{\partial}{\partial x_{j}}\left(\widetilde{u}_{i} \widetilde{u}_{j}\right)=-\frac{1}{\rho} \frac{\partial \widetilde{p}}{\partial x_{i}}+\nu \frac{\partial^{2} \widetilde{u}_{i}}{\partial x_{j} \partial x_{j}}-\frac{\tau_{i j}}{\partial x_{j}}
$$

Similar to RANS Equation (14c) for $\bar{u}_{i}$, the governing LES equations are unclosed and require modelling of the SGS stress tensor $\tau_{i j}$. The type and size of filter is indirectly introduced onto the velocity field though this SGS stress tensor [63]. Applying the decomposition of Equations (24) to the first term on the right side of Equation (33) it can be separated into terms with some physical significance,

$$
\begin{aligned}
\tau_{i j} & =\widetilde{\left(\widetilde{u}_{i}+u_{i}^{\prime}\right)\left(\widetilde{u}_{i}+u_{i}^{\prime}\right)}-\widetilde{u}_{i} \widetilde{u}_{j} \\
\tau_{i j} & =\widetilde{\widetilde{u}_{i} \widetilde{u}_{j}}+\widetilde{\widetilde{u}_{i} u_{j}^{\prime}}+\widetilde{\widetilde{u}_{j} u_{i}^{\prime}}+\widetilde{u_{i}^{\prime} u_{j}^{\prime}}-\widetilde{u}_{i} \widetilde{u}_{j} \\
\tau_{i j} & =\underbrace{\widetilde{u_{j}}-\widetilde{u}_{i} \widetilde{u}_{j}}_{\widetilde{u}_{i j}}+\underbrace{\widetilde{u_{i} u_{j}^{\prime}}+\widetilde{u_{j} u_{i}^{\prime}}}_{C i j}+\underbrace{\widetilde{u_{i}^{\prime} u_{j}^{\prime}}}_{R_{i j}^{\prime}}
\end{aligned}
$$

where $L_{i j}$ is the Leonard stress tensor, $C_{i j}$ is the cross-stress tensor component, and $R_{i j}$ is the Reynolds subgrid tensor. The Leonard term can be computed from the filtered velocity field and represents the interaction between the resolved and SGS scales. The cross-stress relates the (typical) energy transfer from the large filtered and smaller modeled eddies. The Reynolds stress term is analogous to the components of the stress tensor in the RANS formulation, and represents the effect of the small eddy interaction with one another. In comparison to the RANS method, the Leonard and cross stress terms arise from the difference in the double time averaging and double filtering as $\widetilde{\widetilde{\Phi}} \neq \widetilde{\Phi}$. Further discussion on the significance of the stresses is found in Section 13.5.2 of Pope [63]. 
While there were early attempts to model each subgrid stress component independently, all of the stresses are grouped into a single subgrid stress tensor and modeled as a whole. This is mainly due to the preservation of the Galilean invariant (independent of inertial frame) properties of the continuity and momentum equations, since the filtering of individual terms such as the cross and Leonard stresses are not Galilean invariant but the total sum is $[58,60,67]$.

Similar to the problem faced with the RANS momentum equations a model is applied to close Equations (31) and (35) and relate the SGS stress tensor to known flow quantities. The Boussinesq eddy viscosity assumption is once again applied where the SGS stress tensor is proportional to the local filtered rate of strain tensor and SGS viscosity,

$$
\tau_{i j}=-\nu_{S G S}\left(\frac{\partial \tilde{u}_{i}}{\partial x_{j}}+\frac{\partial \tilde{u}_{j}}{\partial x_{i}}\right)+\frac{1}{3} \tau_{i i} \delta_{i j}
$$

while the term $\tau_{i i}$ is included to ensure the sum of the modeled normal SGS stresses are equal to the kinetic energy of the SGS eddies [57] (it is grouped in with the filtered pressure term and therefore does not require additional modeling). However, a sub-grid scale model is required for the new unknown sub-grid scale eddy-viscosity $\nu_{S G S}$. Similar to the requirements of a RANS turbulence model, a SGS model is used to close the system of equations and imitate the energy cascade energy drain on the resolved flow.

In this work the one-equation eddy viscosity model, with one variant given by 
Yoshizawa and Horiuti [68] is used to relate a characteristic length scale and a velocity scale of SGS eddy size. This model improves the equilibrium assumption of the original Smagorinsky model which becomes less accurate for flow conditions such as free shear layers, separating and reattaching flows, boundary layers and wall dominated domains like pipes and channels [58]. In addition to, this the one-equation model possesses the ability to predict backscatter and has higher numerical stability making it computationally easier than the Smagorinsky model [69]. By using the definition of the velocity scale the one-equation eddy model has shown to perform better to model transitional or large scale unsteadiness flows in comparison to an algebraic relation [58]. The length scale is taken to be the filter width, or grid spacing, while the velocity scale is taken as the square root of the specific SGS turbulent kinetic energy $\sqrt{k_{S G S}}$ such that,

$$
\nu_{S G S}=C_{k} \Delta \sqrt{k_{S G S}}
$$

Analogous to RANS like turbulent modeling, a transport equation is introduced to account for the effects of convection, diffusion, production and destruction of this energy,

$$
\frac{k_{s g s}}{\partial t}+\widetilde{u}_{j} \frac{\partial k_{s g s}}{\partial x_{j}}=\frac{\partial}{\partial x_{j}}\left[\left(\nu_{s g s}+\nu\right) \frac{\partial k_{s g s}}{\partial x_{j}}\right]-\tau_{i j}\left(\frac{\partial \widetilde{u}_{i}}{\partial x_{j}}+\frac{\partial \widetilde{u}_{j}}{\partial x_{i}}\right)-C_{\varepsilon}\left(k_{s g s}^{3 / 2} / \Delta\right)
$$

where the specific SGS kinetic energy dissipation rate is defined as,

$$
\varepsilon_{S G S}=C_{\varepsilon}\left(k_{S G S}^{3 / 2} / \Delta\right)
$$

Lastly the equations are closed with the standard one-equation eddy constants,

$$
C_{k}=0.094 \quad C_{\varepsilon}=1.048
$$




\section{Chapter 3}

\section{Grid Refinement Study}

\subsection{Computational Domain}

A computational grid is generated with OpenFOAM's native meshing utility snappyHexMesh which produces a 3-dimensional mesh consisting of hexahedra and split-hexahedra elements. The utility operates by edge splitting local cells of the block background mesh and snapping them to the edges of the surface geometry in Stereolithography (.stl) format [56]. The cells located inside the geometry are then removed to produce the Cartesian hex mesh. The size of the domain is generated following the standards outlined in the COST Action 732 [70] and the Agriculture Institute of Japan (AIJ) guidelines [71] for using CFD to simulate flows in urban environments. The domain sizing of the single square building and the boundary conditions are shown in Figure 16, where $R_{\|}$is the wind parallel side length.

A mapped inlet boundary condition is used to generate synthetic transient inlet turbulence. The velocity components are sampled at a plane 12 building widths 

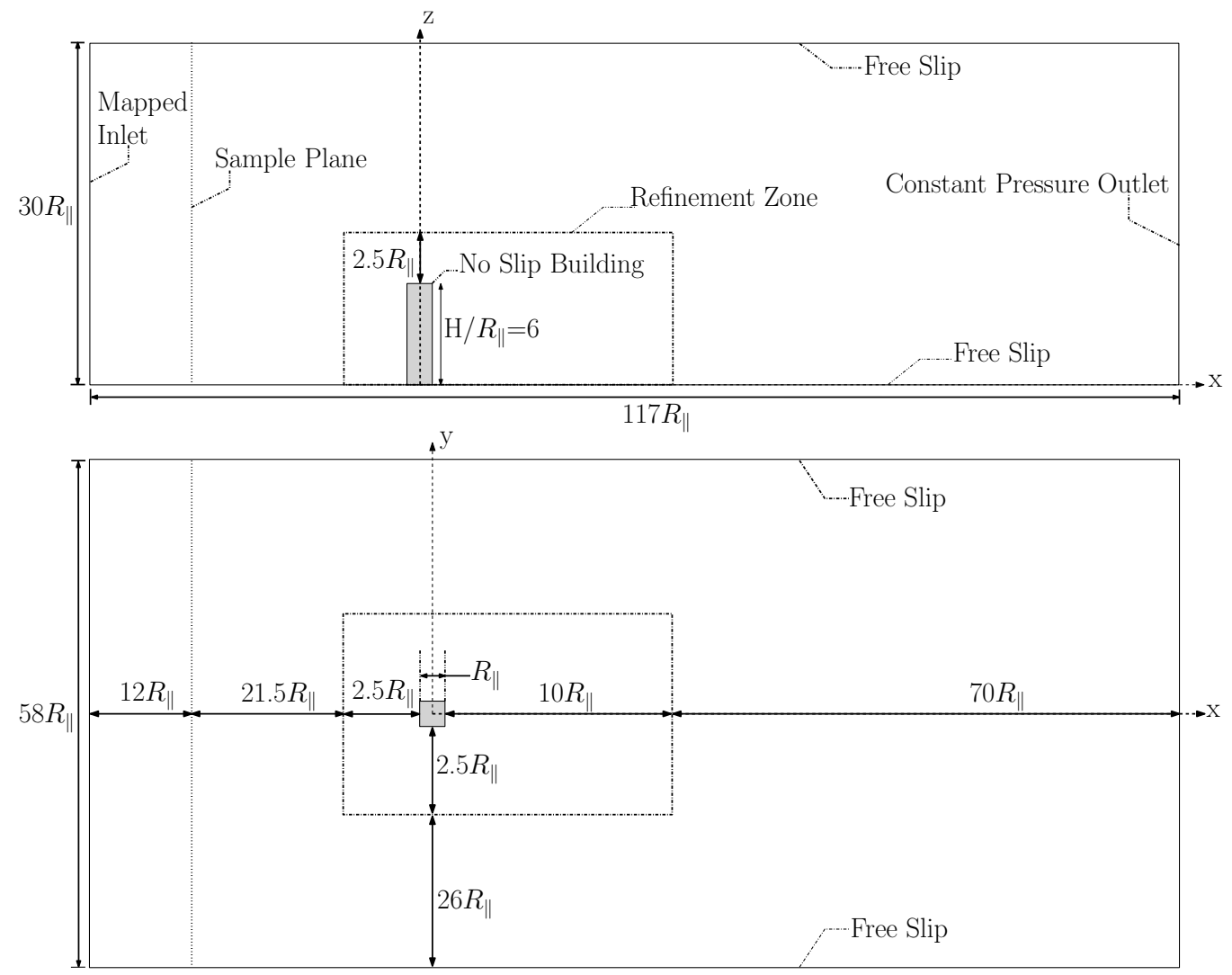

Figure 16: Computational domain, boundary conditions and wake refinement zone downstream from the inlet, scaled to ensure the bulk flow rate is constant, and applied to the inlet plane [58]. A free slip boundary condition is applied to the ground, side walls and top. Local grid refinement is used to reduce the computational cost and limit the most refined region to the wake as shown in Figure 17. This introduces a commutation error which can produce errors on the order of the SGS stress [58] due to the changing filter width which violates the commutation with differentiation assumption (which requires a constant filter size). To minimize the influence of this error, gradual levels of successive refinement are used to reduce sudden changes in the filter width (shown in Figures 17a and 17b). The refinement boundaries are also 
placed away from the regions of direct interest (near the building and within the wake) as the error will be greatest at the refinement boundaries and will reduce as the distance from these refinement boundaries increases [58].

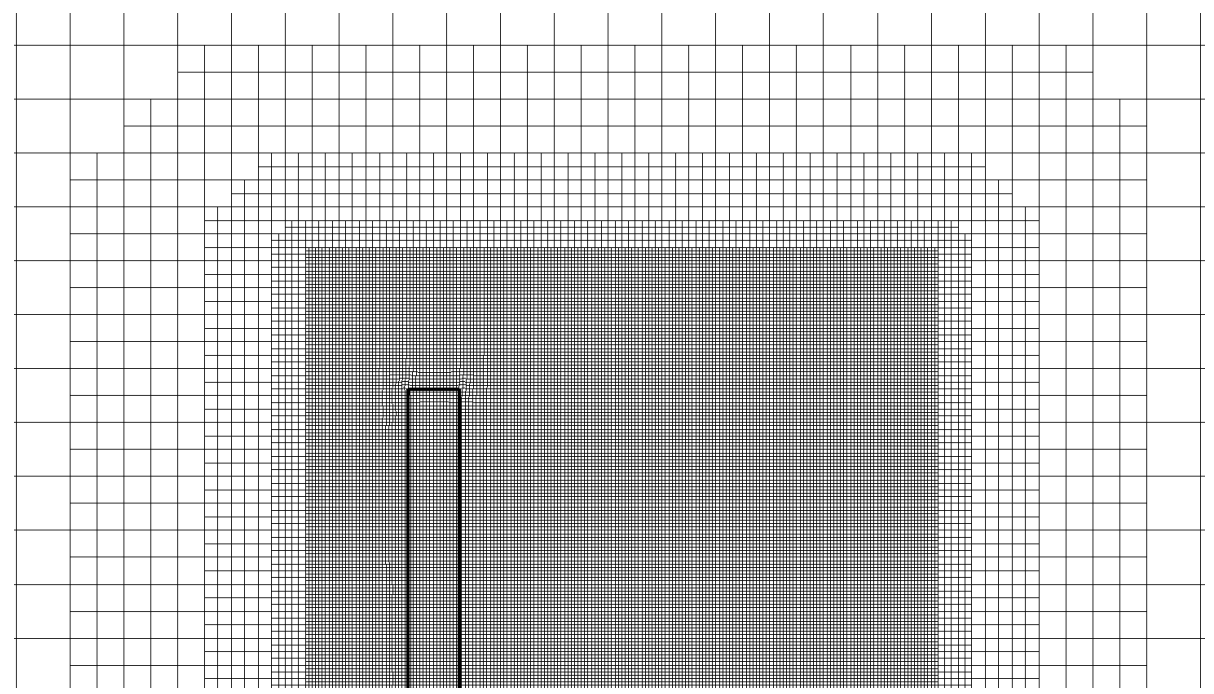

(a) Side view, $\mathrm{X}-\mathrm{Z}$ plane

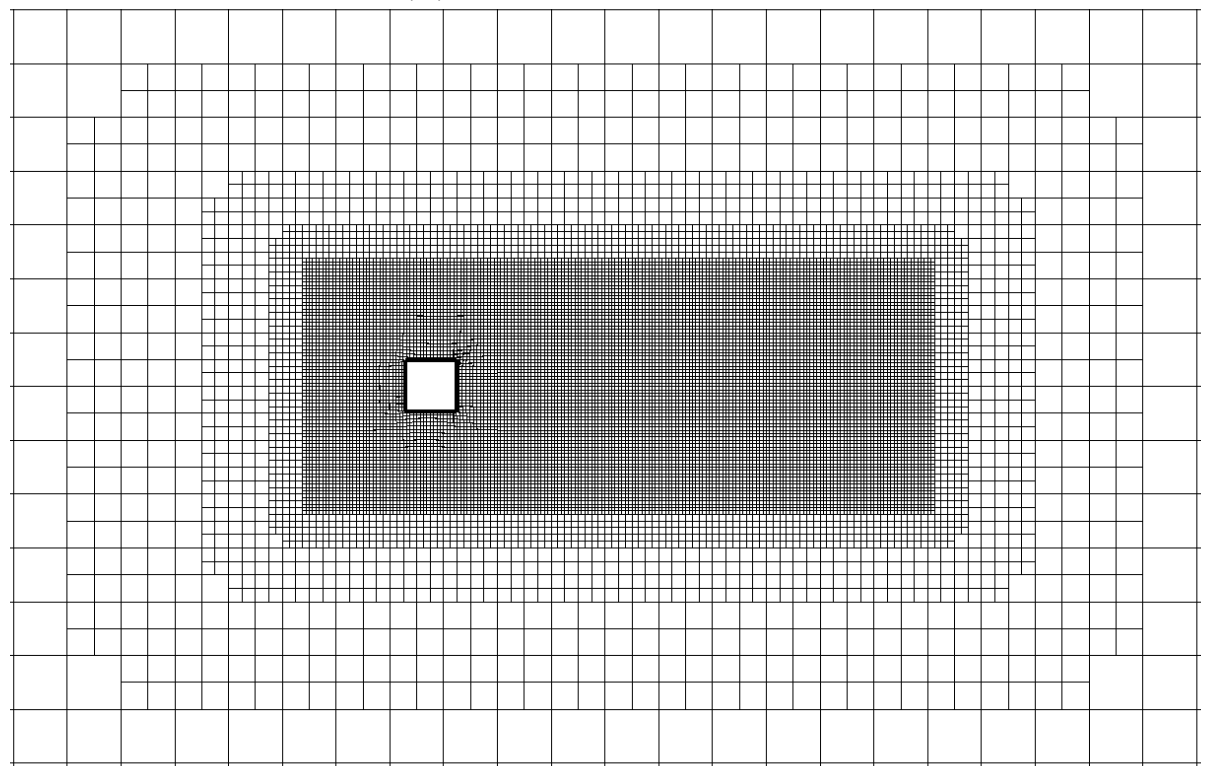

(b) Top view, Y-Z plane

Figure 17: Cartesian hex mesh with gradual cell refinement 


\subsection{LES Verification and Validation Study}

In this work the errors associated with spatial discretization are assessed by comparing time averaged velocity profiles across the wake and by calculating the LES Index Quality [72] (LES_IQ) between successive levels of grid refinement. A grid independent solution with LES is more difficult to determine than for RANS simulations in that the degree of model approximation applied, in addition to the numerical accuracy of that model, depends directly on the grid size [73] through the filter width. Therefore, the LES_IQ uses results from two different grid densities to perform a Richardson extrapolation for establishing how the results compare to a theoretical solution in which the filter width is so small as to eliminate the use of the sub-grid scale approximation. It is then possible to establish what percentage of the turbulence is modelled directly in the LES simulations for each grid (and conversely how much of the turbulence is represented by the SGS model).

Table 1: Refined area grid details

\begin{tabular}{cccc}
\hline \hline Name & \# building cells & Total \# cells & CPU Hours \\
\hline Coarse & $10 \mathrm{x} 10 \mathrm{x} 60$ & $1.13 \times 10^{6}$ & 0.838 \\
Medium & $15 \mathrm{x} 15 \mathrm{x} 90$ & $2.43 \times 10^{6}$ & 3.383 \\
Fine & $22 \times 22 \times 132$ & $7.67 \times 10^{6}$ & 17.696 \\
\hline \hline
\end{tabular}

Three grids of increasing density are used for verification purposes. Each successive refinement increases the node count on the building surface by $50 \%$ in each direction as summarized in Table 1 . The coarsest grid has 10 nodes along the building side parallel to the freestream wind $\left(R_{\|}\right)$while in the finest grid this value is increased to 22 . The computational time shown is that required to generate 30 seconds of LES results. 
The first 6 seconds, approximately 1 flow through of the refined wake area, are used to allow the mapped flow field from a steady RANS simulation to transition into the domain and produce coherent vortical structures, while the flow is then time averaged over the remaining 24 seconds. All verification and validation comparisons are performed across three vertical and three horizontal lines downstream of the building as shown in Figure 18. These locations are a subset of measurement locations used in the wind tunnel experiments of Meng and Hibi [74].

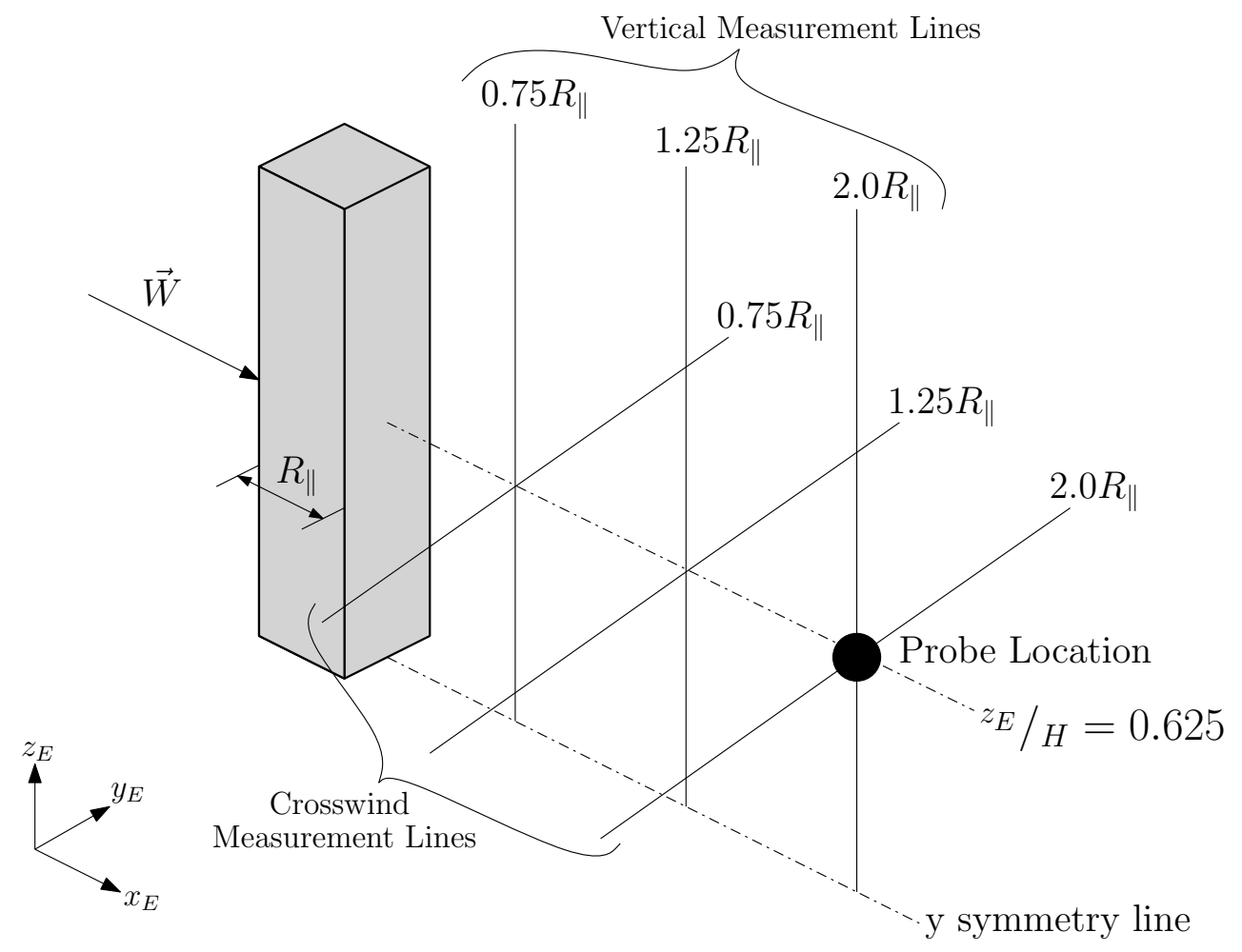

Figure 18: Data sample and plotting locations 


\subsubsection{Q-Criterion Turbulence Visualization}

The concept of a vortex is intuitive however a formal definition, and visualization methods, are not as straightforward. A study by Jeong and Hussain [75] shows the downfall of using either a local pressure minimum, streamlines, or vorticity magnitude as measure for a vortex (also see de Villers [58]). The result of the study concluded the vortex core can be defined with complex eigenvalues of the velocity's divergence $(\nabla \cdot V)$, and satisfies the characteristic equation,

$$
\lambda^{3}+\boldsymbol{P} \lambda^{2}+\boldsymbol{Q} \lambda-\boldsymbol{R}=0
$$

For incompressible flow the first invariant $\boldsymbol{P}$ is zero $(\nabla \cdot V=0)$ and the third invariant $\boldsymbol{R}$ is equal to the determinant of $\nabla \cdot V$. The second invariant $\boldsymbol{Q}$ is defined as,

$$
\boldsymbol{Q}=\frac{1}{2}\left(\Omega_{i j} \Omega_{i j}-S_{i j} S_{i j}\right)
$$

where $S_{i j}$ is rate-of-strain tensor and $\Omega_{i j}$ is the rate-of-rotation tensor (vorticity tensor), which are the symmetric and antisymmetric parts of $\nabla \cdot V$ respectively and defined as,

$$
\begin{aligned}
S_{i j} & =\frac{1}{2}\left(\frac{\partial u_{i}}{\partial x_{j}}+\frac{\partial u_{j}}{\partial x_{i}}\right) \\
\Omega_{i j} & =\frac{1}{2}\left(\frac{\partial u_{i}}{\partial x_{j}}-\frac{\partial u_{j}}{\partial x_{i}}\right)
\end{aligned}
$$

This Q-criterion, proposed by Hunt et al [76], defines a vortex as a connected fluid region with a positive second invariant of the velocity gradient tensor $\nabla \cdot V[77]$. When $\boldsymbol{Q}$ is positive it represents locations in the flow were the rotation dominates the strain and shear [76-78]. While not a measure of grid quality, the Q-criterion 
provides the ability to visualize vortical structures as isosurfaces as shown in Figure 19. As the grid spacing is successively reduced, finer and smaller vortical structures are captured resulting in an increase in the amount of directly resolved vortical structures. This visualization provides an initial estimate that the coarse grid has inadequate density resolving the desired level of turbulent motions.

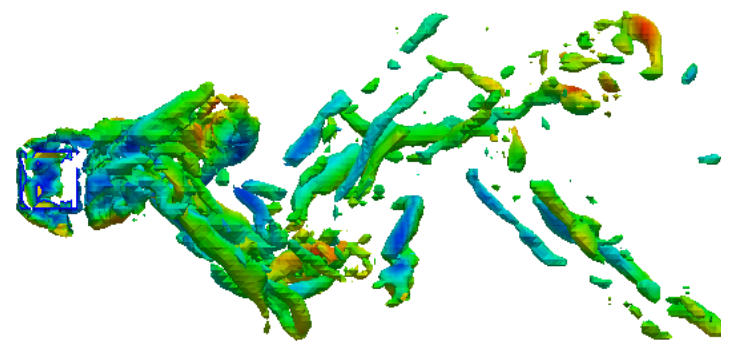

(a) Coarse mesh, top view

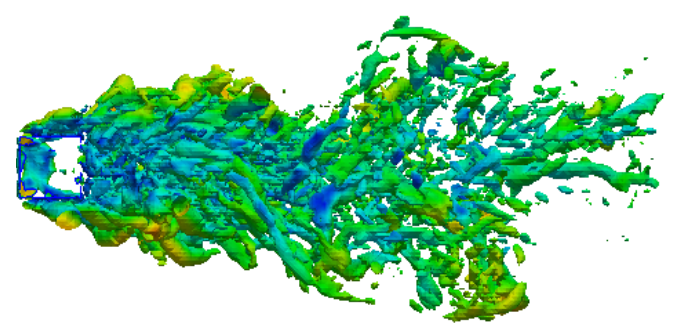

(c) Medium mesh, top view

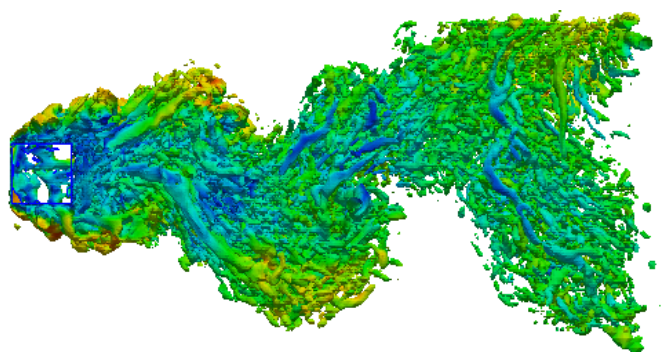

(e) Fine mesh, top view

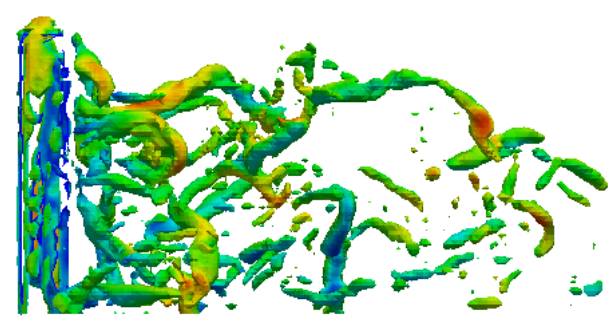

(b) Coarse mesh, side view

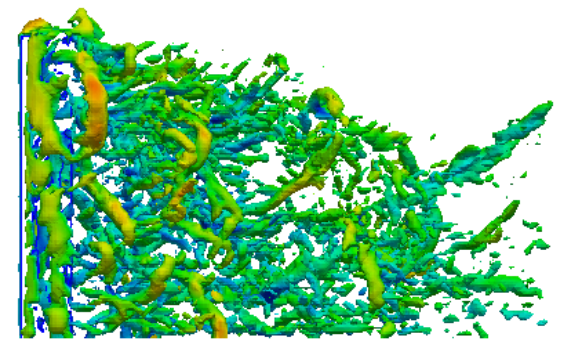

(d) Medium mesh, side view

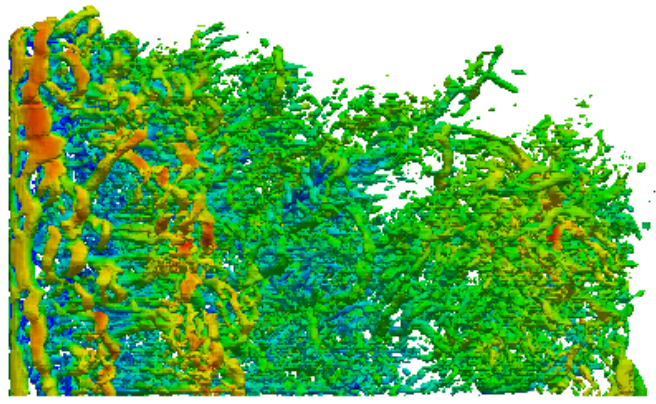

(f) Fine mesh, side view

Figure 19: $Q$-criterion vortex visualization with $Q=200$ isosurfaces, $\mathrm{t}=30 \mathrm{~s}$. 


\subsubsection{Time Averaged Velocity Profile}

The traditional single-grid estimator of comparing the time averaged velocity profiles is first used to study the change in numerical results across the changing grid size. The velocity profiles are compared for each grid density along the six measurement lines outlined in Figure 18.

The velocities along the vertical measurement lines at $x / R_{\|}=0.75$ downstream of the building, the results shown in Figure 20a, illustrate a slightly larger average difference between the coarse and medium grids (an average difference of 4.51\%) than between the medium and fine grids (an average difference of 3.81\%). While all three grid densities resolve similar velocity magnitudes in the wake of the building, $z / H<1$, the fine grid predicts a change in velocity across the upper edge of the wake (between approximately $z / H=0.9$ and $z / H=1.0$ as highlighted by the insert in Figure 20a) of $17.3(\mathrm{~m} / \mathrm{s}) / \mathrm{m}$ while the same slope is $22.2(\mathrm{~m} / \mathrm{s}) / \mathrm{m}$ and 29.4 $(\mathrm{m} / \mathrm{s}) / \mathrm{m}$ when using the medium and coarse grids respectively.

In the direction parallel to the ground passing through the wake at a height of $62.5 \%$ of the building height (i.e. $z / H=0.625$ ) the results from all three grids are in close agreement (Figure 26b). The percent difference in peak velocity at the center of the wake between the coarse and medium grid is $34 \%$ and $15.7 \%$ between the medium and fine mesh. The gradients at the edge of the wake, highlighted by the insert in Figure 20b, are also in close agreement between the medium and fine grids where a value of $28.6(\mathrm{~m} / \mathrm{s}) / \mathrm{m}$ is predicted using the medium grid and $30.0(\mathrm{~m} / \mathrm{s}) / \mathrm{m}$ is predicted using the fine grid, a difference of $4.9 \%$. In this case, the coarse grid again 
shows a larger discrepancy in that the velocity gradient at the wake edge is predicted to be $26.6(\mathrm{~m} / \mathrm{s}) / \mathrm{m}$ a difference of $7.5 \%$ from the medium mesh.

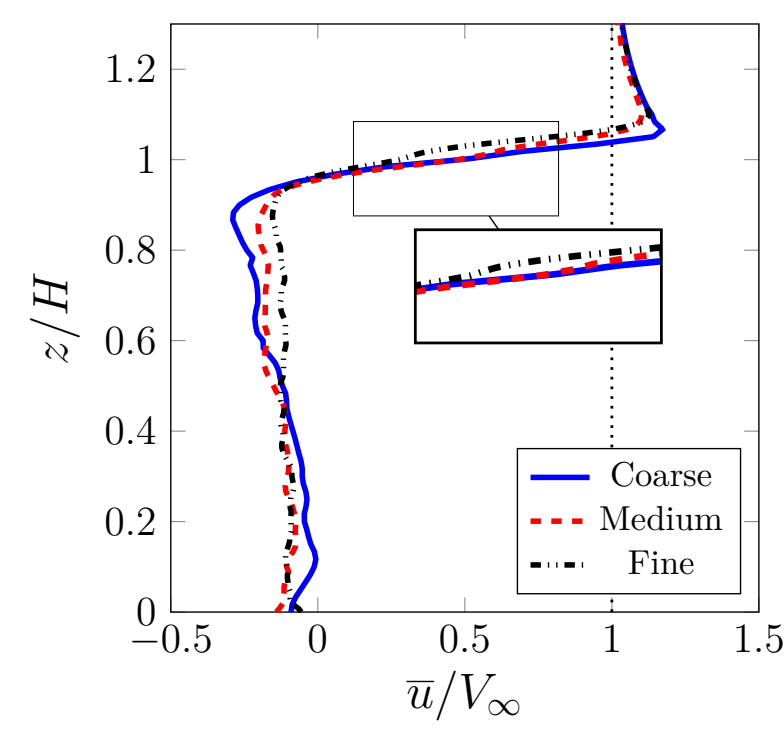

(a) Vertical measurement line

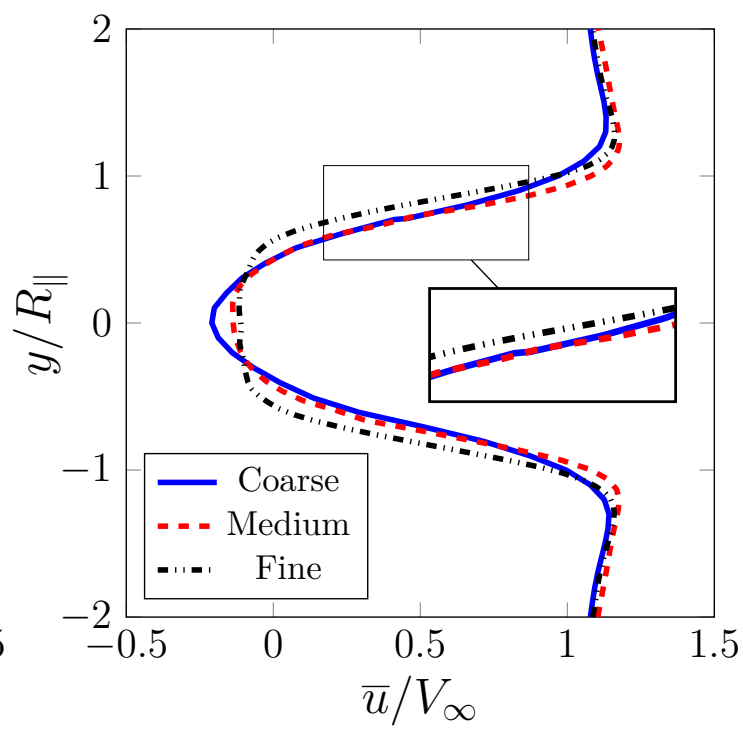

(b) Crosswind measurement line

Figure 20: Time averaged streamwise velocity profiles at a distance $x / R_{\|}=0.75$ downstream of the building

Along the vertical measurement line $x / R_{\|}=1.25$ downstream of the building, similar trends are found, as Figure 21a illustrates a larger difference between the coarse and medium grids (an average difference of $13.3 \%$ ) than between the medium and fine grids (an average difference of 3.5\%). Studying the wake, beneath approximately $z / H=0.6$, it can be noted that the course grid does not predict any recirculation except near the building rooftop, whereas both the medium and fine grids predict a recirculation zone behind the entire height of the building as evidenced by the negative value of $\bar{u} / V_{\infty}$. This recirculation zone in the wake is expected from the experimental results shown in Figure 27a of Section 3.2.4. The fine grid predicts a change in velocity across the upper edge of the wake (between approximately 
$z / H=0.9$ and $z / H=1.0$ as highlighted by the insert in Figure 21a) of 11.6 $(\mathrm{m} / \mathrm{s}) / \mathrm{m}$ while the same slope is $15.0(\mathrm{~m} / \mathrm{s}) / \mathrm{m}$ and $20.7(\mathrm{~m} / \mathrm{s}) / \mathrm{m}$ when using the medium and coarse grids respectively. The upper edge of the wake (as defined as the point where $\bar{u} / V_{\infty}=1$ ) is calculated to be $z / H=1.03, z / H=1.08$, and $z / H=1.07$ for the coarse, medium, and fine grids respectively. This is illustrated in Figure 21a with the vertical dotted line. Additionally, the coarse grid has a slight oscillation in this region unseen in both the medium and fine grids.

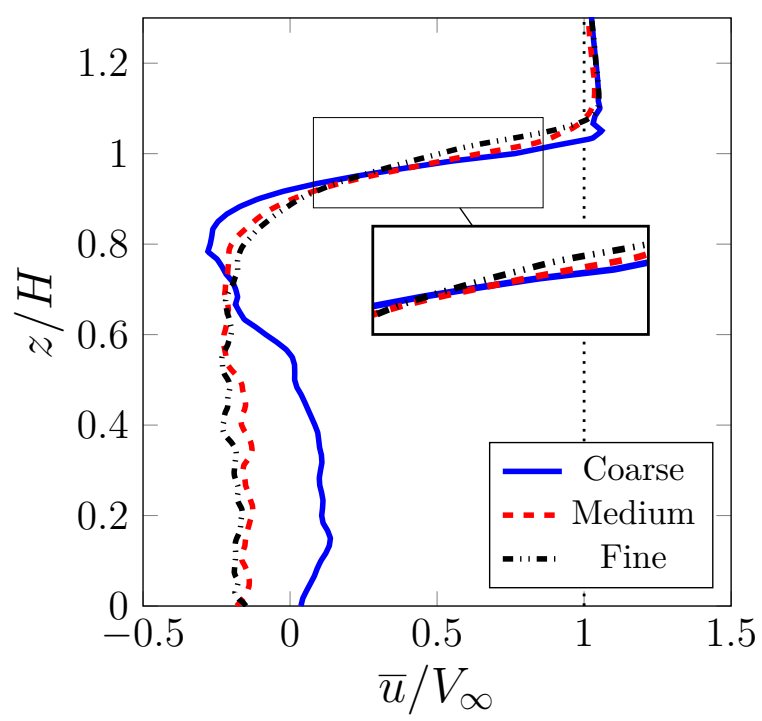

(a) Vertical measurement line

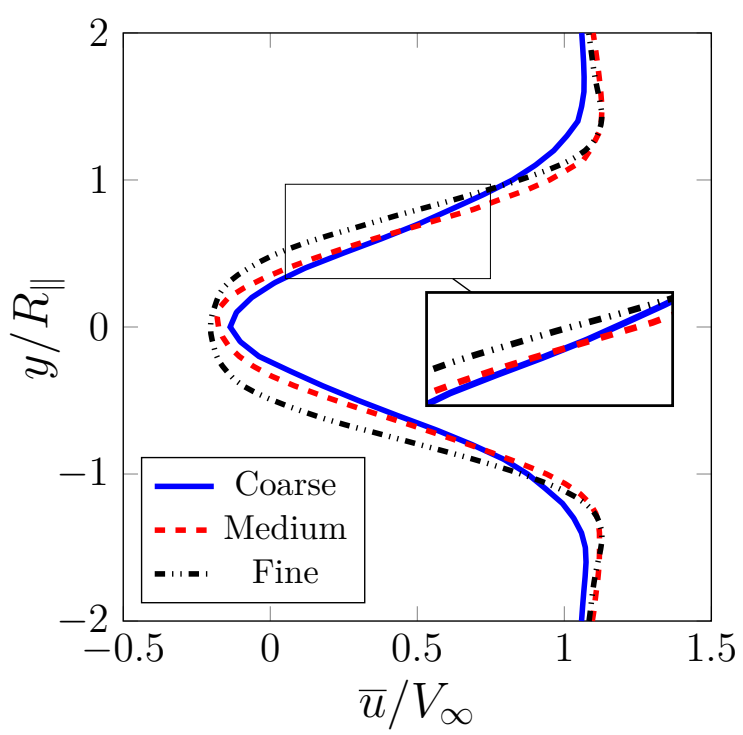

(b) Crosswind measurement line

Figure 21: Time averaged streamwise velocity profiles at a distance $x / R_{\|}=1.25$ downstream of the building

For the horizontal measurement line passing through the wake at a height of $62.5 \%$ of the building height (i.e., $z / H=0.625$ ) the results from all three grids are in close agreement. The minimum velocity at the center of the wake using the medium grid is within $13.4 \%$ of that obtained using the fine grid, while the coarse grid yields a minimum that is $32.9 \%$ away from the fine grid. The gradients at the edge of the 
wake, highlighted by the insert in Figure 21b, are also in close agreement between the medium and fine grids where a value of $20.7(\mathrm{~m} / \mathrm{s}) / \mathrm{m}$ is predicted using the medium grid and $22.1(\mathrm{~m} / \mathrm{s}) / \mathrm{m}$ is predicted using the fine grid, a difference of $6.8 \%$. In this case, the coarse grid again shows more of a discrepancy in that the velocity gradient at the wake edge is predicted to be $15.8(\mathrm{~m} / \mathrm{s}) / \mathrm{m}$ which is $23.7 \%$ below the medium grid result.

When the same comparison is applied to the vertical measurement line $x / R_{\|}=2.00$ downstream it is seen from Figure 22a that the course grid greatly under predicts the velocity and has a large oscillations around $z / H=0.6$ and small oscillation around $z / H=1$, both unseen in the medium and fine grids. Overall the average difference between the coarse and medium meshing is $15.6 \%$, while between the medium and fine is $6.6 \%$. The slopes found as highlighted by the insert in Figure 22a, are $7.6(\mathrm{~m} / \mathrm{s}) / \mathrm{m}, 6.8(\mathrm{~m} / \mathrm{s}) / \mathrm{m}$, and $6.4(\mathrm{~m} / \mathrm{s}) / \mathrm{m}$ for the coarse, medium, and fine meshes respectively. This corresponds to a $10.5 \%$ increase from the coarse to medium grids with only $5.9 \%$ change from medium to fine.

Along the horizontal measurement line at $x / R_{\|}=2.00$, the fine mesh resolves a much lower peak velocity with a $41 \%$ difference between the course and medium mesh in contrast to a $22 \%$ difference between the medium and fine meshes. The wake edge velocity gradients are found to be $9.3(\mathrm{~m} / \mathrm{s}) / \mathrm{m}, 13.1(\mathrm{~m} / \mathrm{s}) / \mathrm{m}$, and $16.0(\mathrm{~m} / \mathrm{s}) / \mathrm{m}$ for the coarse, medium, and fine grids respectively. 


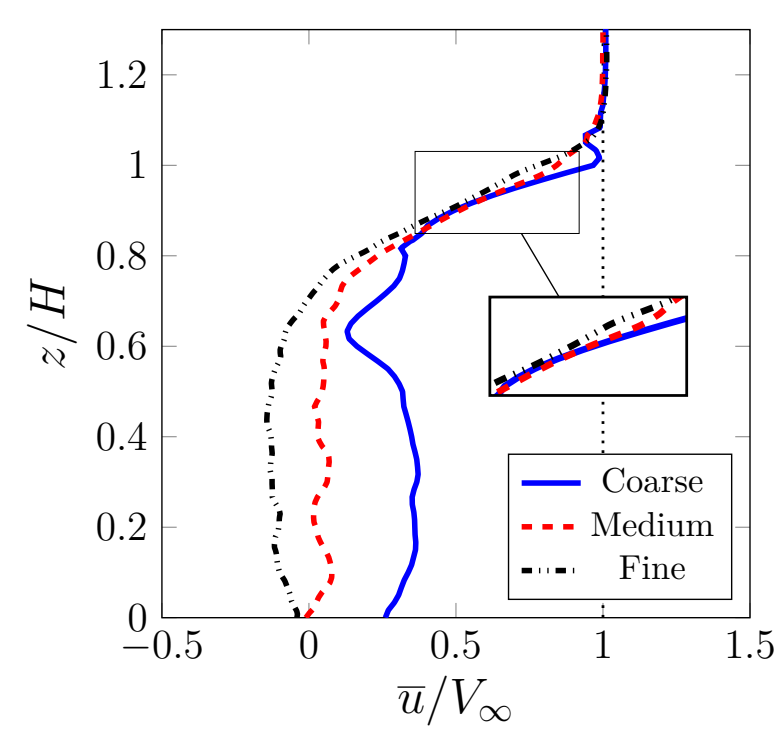

(a) Vertical measurement line

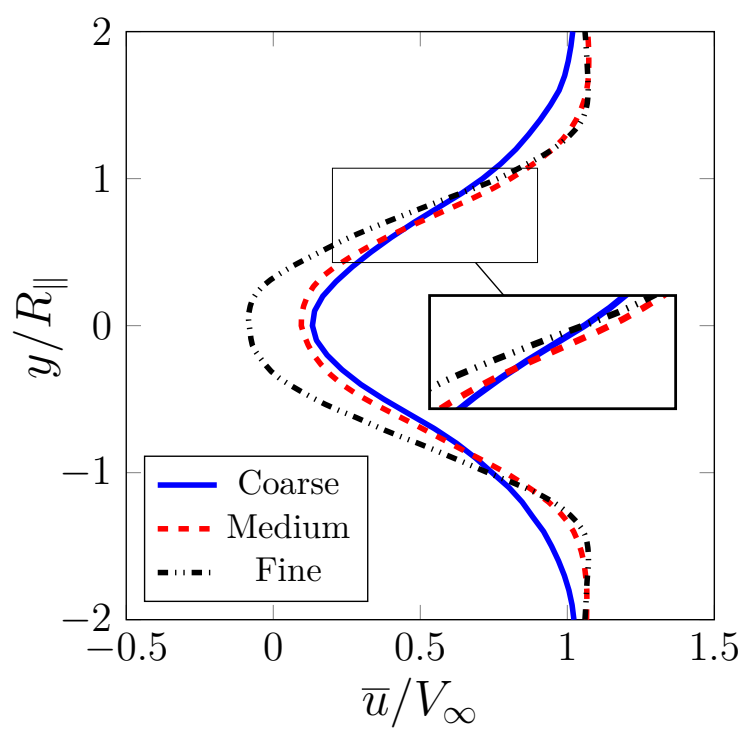

(b) Crosswind measurement line

Figure 22: Time averaged streamwise velocity profiles at a distance $x / R_{\|}=2.00$ downstream of the building

\subsubsection{LES Index Quality}

A second grid independence technique is performed in the form of a multi-grid estimator based on the resolved turbulent kinetic energy (TKE). A multi-grid estimator uses the results from at least two different grid densities and a Richardson extrapolation to study how the results compare to the theoretical exact solution. The LES Index Quality (LES_IQ) is a ratio of the resolved TKE to the total [72],

$$
L E S \_I Q_{k}=\frac{k^{r e s}}{k^{t o t}}=1-\frac{\left|k^{t o t}-k^{r e s}\right|}{k^{t o t}}
$$

where $k^{r e s}$ is the LES resolved TKE and $k^{\text {tot }}$ is the theoretical maximum TKE. This maximum is defined as the sum of the resolved scale and the sub-grid scale turbulence 
which can be represented as,

$$
k^{\text {tot }}=k^{r e s}+a_{k} h^{p}
$$

where $a_{k}$ is determined using a Richardson extrapolation, $h$ is a characteristic of the grid quality, and $p$ is the order of accuracy of the numerical scheme. For a finite volume method the characteristic grid quality $h$ can be taken as the cube root of the cell volume. In this work the order of accuracy of the LES numerical simulations is two. The extrapolation constant between two different grid densities can be expressed as,

$$
a_{k}=\frac{1}{h_{2}^{p}}\left[\frac{k_{2}^{\text {res }}-k_{1}^{\text {res }}}{\left(h_{1} / h_{2}\right)^{p}-1}\right]
$$

where the subscript corresponds to a grid of given density (the higher number represents a more dense grid). The index quality for each grid, at a specific spatial location, is then defined as,

$$
\begin{aligned}
& L E S \_I Q_{k}^{1}=1-\frac{\left|a_{k} h_{1}^{p}\right|}{k_{1}^{r e s}+a_{k} h_{1}^{p}} \\
& L E S \_I Q_{k}^{2}=1-\frac{\left|a_{k} h_{2}^{p}\right|}{k_{2}^{r e s}+a_{k} h_{2}^{p}}
\end{aligned}
$$

The LES Index Quality is unity (1) when all turbulent motion scales are resolved directly. However, since LES filters out the TKE at high wavenumbers this ratio is less than unity for a finite filter width. In general, when less than $20 \%$ of the TKE is approximated using the SGS model, a LES simulation is judged to be of sufficient quality $[63,72]$ (under these circumstances the LES_IQ would be $\geq 0.80$ ). The calculated LES_IQ along the same horizontal and vertical measurement lines 
in Figure 18 are shown in Figures 23, 24, 25. From these it is seen the coarsest grid is far too sparse to capture even $40 \%$ at most and produces unphysical extrapolation results. With a doubling of grid density the medium mesh resolves a range from $55-80 \%$, and the fine mesh is almost always above $80 \%$ in all locations.

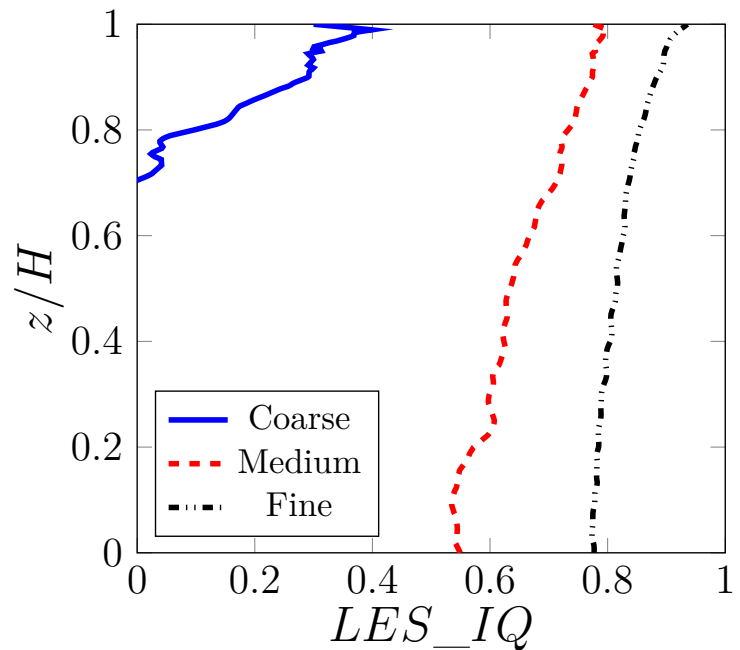

(a) Vertical measurement line

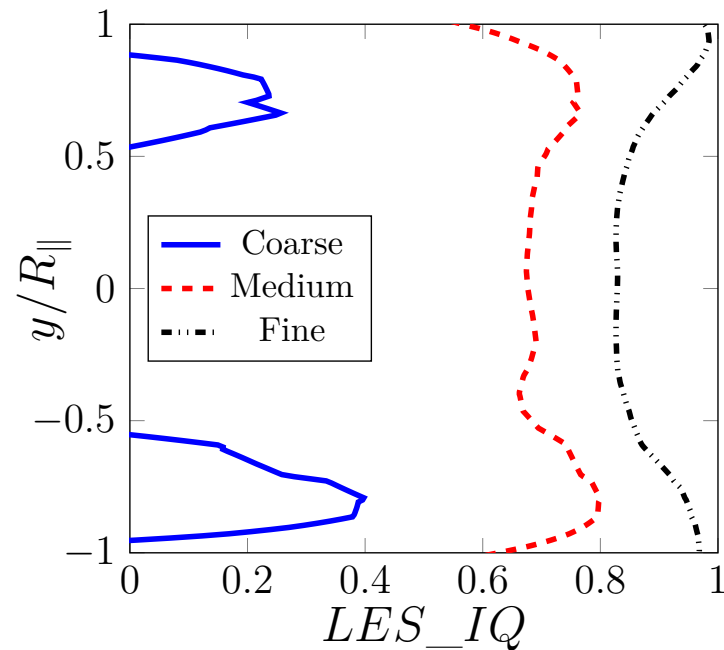

(b) Crosswind measurement line

Figure 23: LES_IQ at $x / R_{\|}=0.75$ downstream of the building

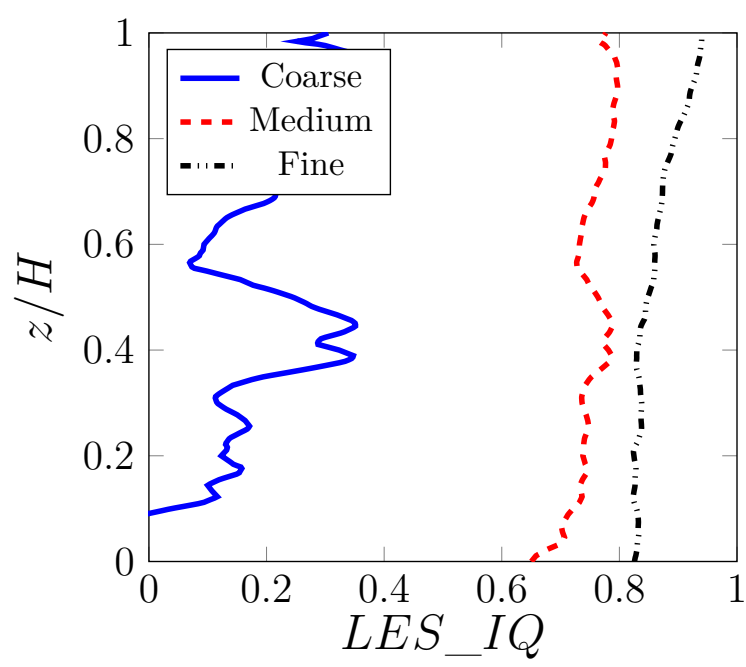

(a) Vertical measurement line

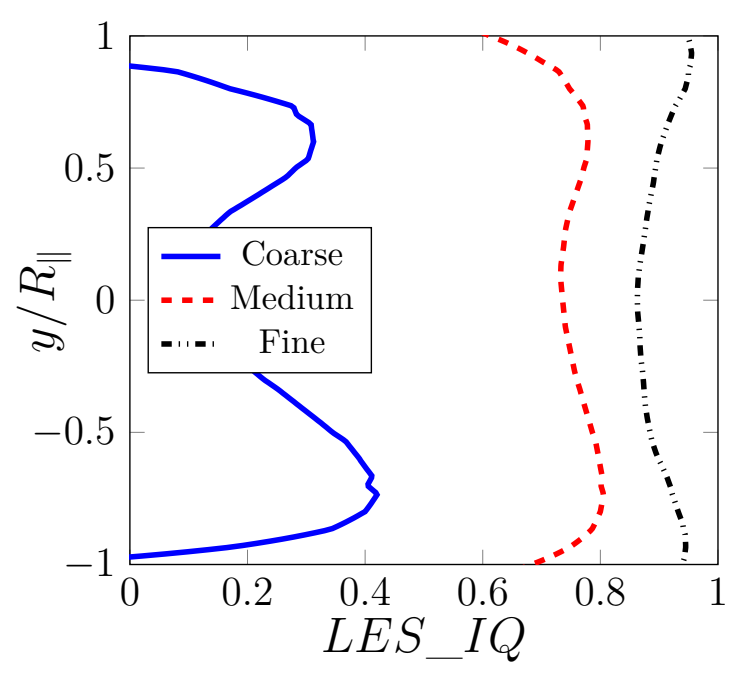

(b) Crosswind measurement line

Figure 24: LES_IQ at $x / R_{\|}=1.25$ downstream of the building 


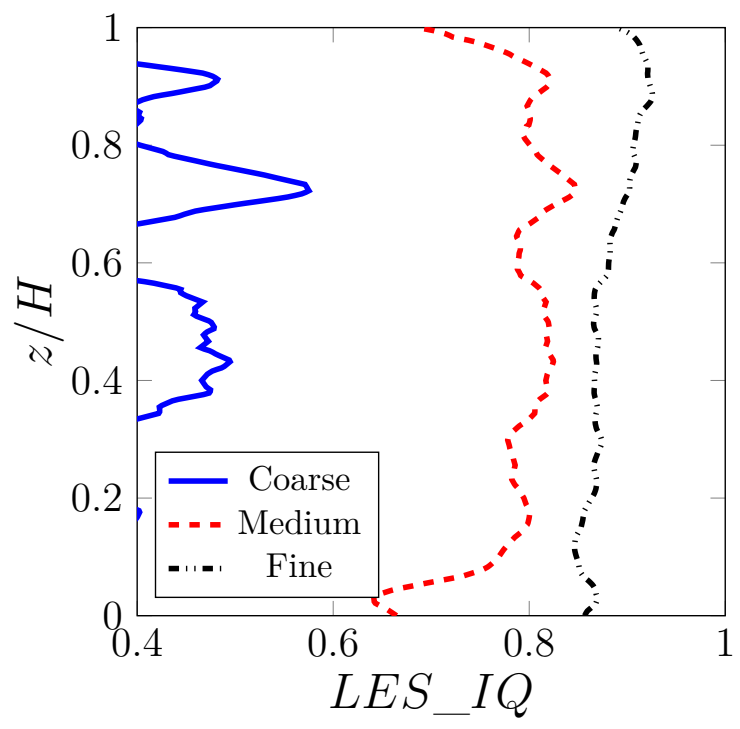

(a) Vertical measurement line

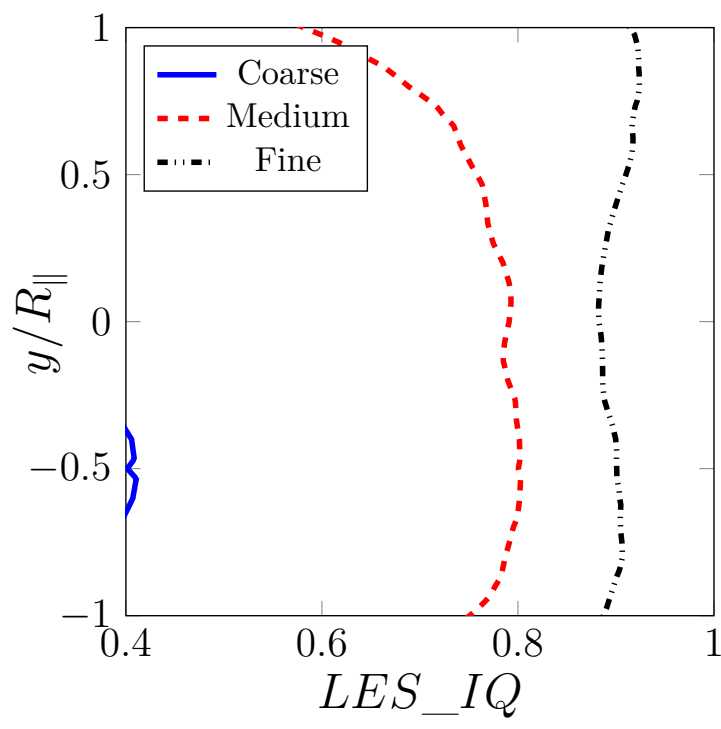

(b) Crosswind measurement line

Figure 25: LES_IQ at $x / R_{\|}=2.00$ downstream of the building

\subsubsection{Experimental Comparison}

Based on the above results of the time averaged velocity profiles and LES_IQ, the fine grid density is chosen to validate the LES simulations against the experimental results of Meng and Hibi [74]. The simulated flow conditions are modified slightly in that the building now has dimensions of $0.04 \mathrm{~m}$ by $0.04 \mathrm{~m}$ by $0.08 \mathrm{~m}$ (the height to characteristic length is reduced to 1.41 from the grid convergence study value of 4.24) and the Reynolds number is reduced to $1.69 \times 10^{4}$ based on the characteristic length D (This value corresponds to the height based Reynolds number of $2.4 \times 10^{4}$ used in Meng and Hibi [74]). The same time averaging process used for the grid verification study is applied to these experimental results and the extracted velocity profiles from the LES simulations at $x / R_{\|}=0.75$ are shown in Figures 26a and 26b. The average error is found through the mean of the errors between each experimental 


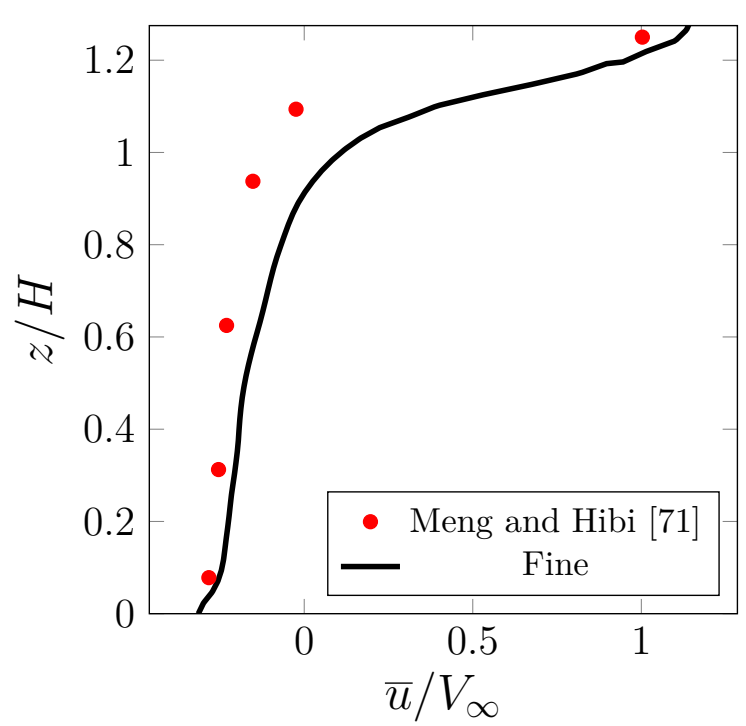

(a) Vertical measurement line

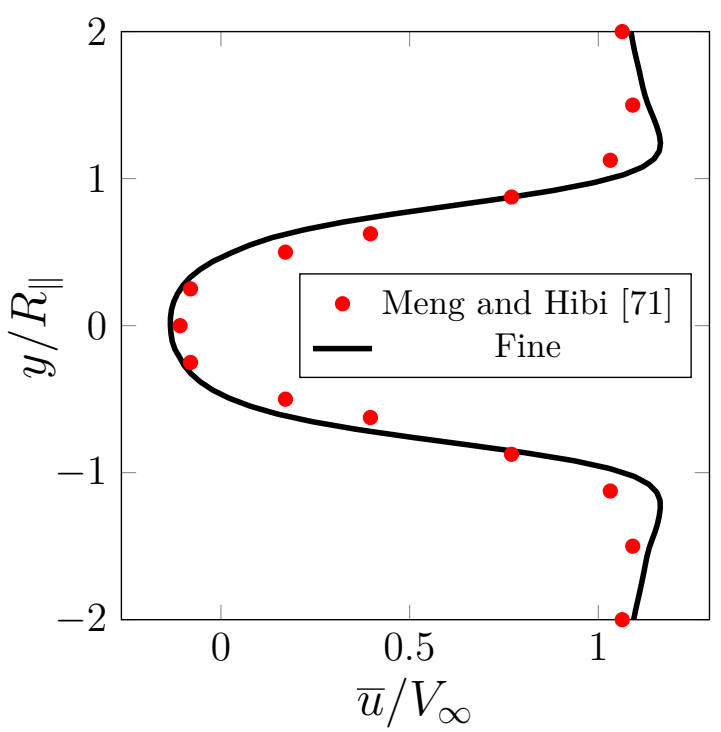

(b) Crosswind measurement line

Figure 26: Experimental vs. numerical time averaged streamwise velocity comparison at a distance of $x / R_{\|}=0.75$ downstream of the building.

point and the numerical value at the same spatial location. For the vertical and horizontal measurement lines at $x / R_{\|}=0.75$ the average differences are $9.7 \%$ and 17.1\%. Similarly the results of the measurement lines at $x / R_{\|}=1.25$ and $x / R_{\|}=$ 2.00 are shown in Figures 27, and 28. The average error between the experimental and numerical results for each respective position are $12.9 \%, 17.6 \%, 9.7 \%$, and $17.4 \%$. From these figures an acceptable level of agreement between the experimental and numerical results is observed where the numerical results always underpredict the vertical velocity profile and over predict the horizontal profiles. 


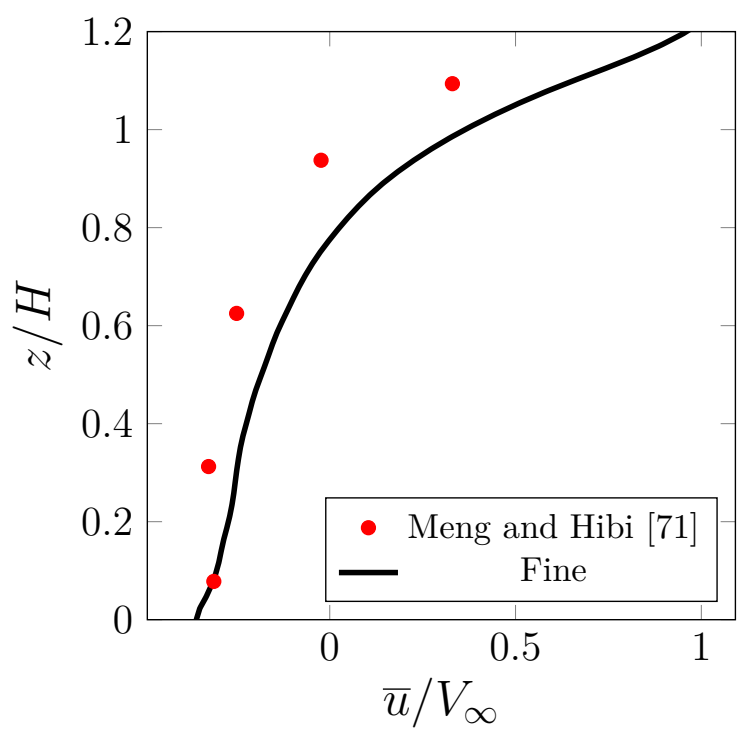

(a) Vertical measurement line

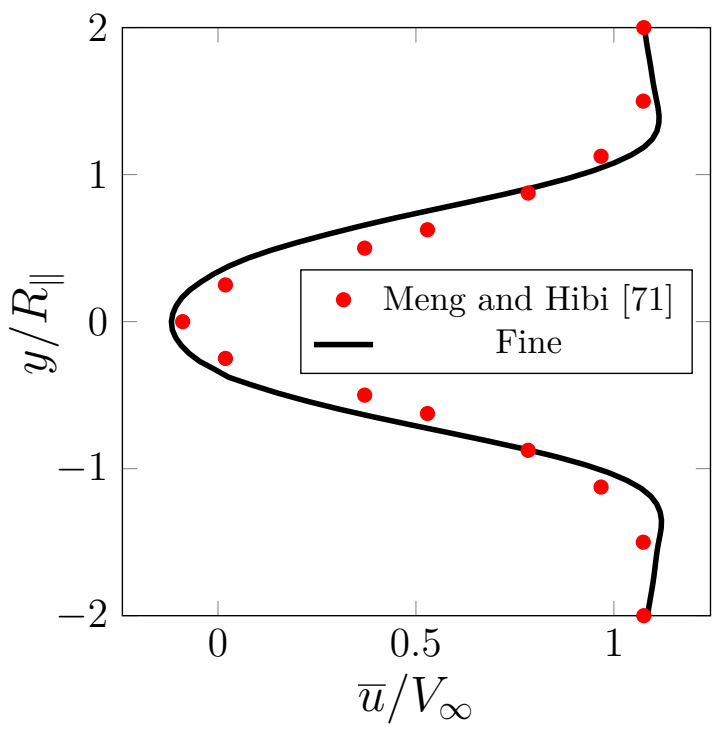

(b) Crosswind measurement line

Figure 27: Experimental vs. numerical time averaged streamwise velocity comparison at a distance of $x / R_{\|}=1.25$ downstream of the building.

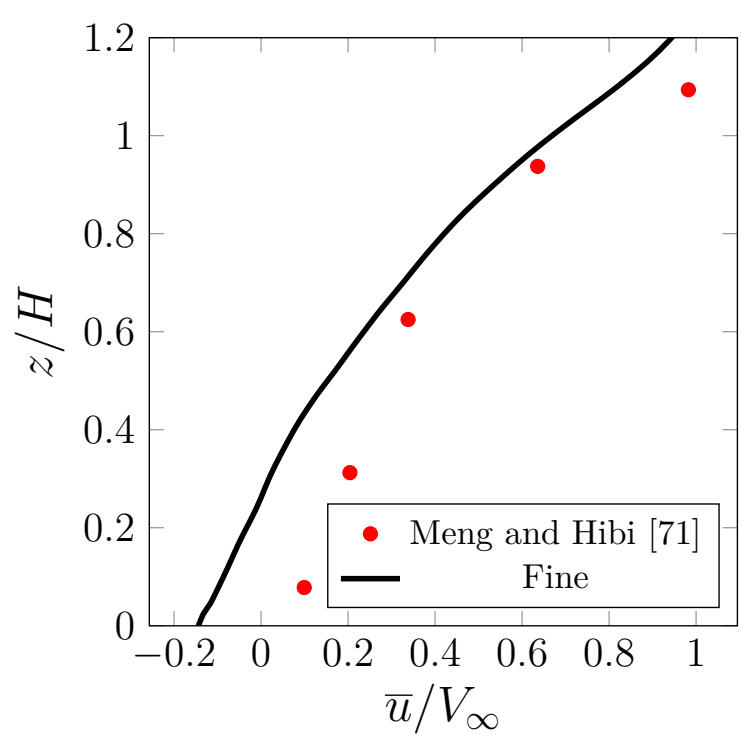

(a) Vertical measurement line

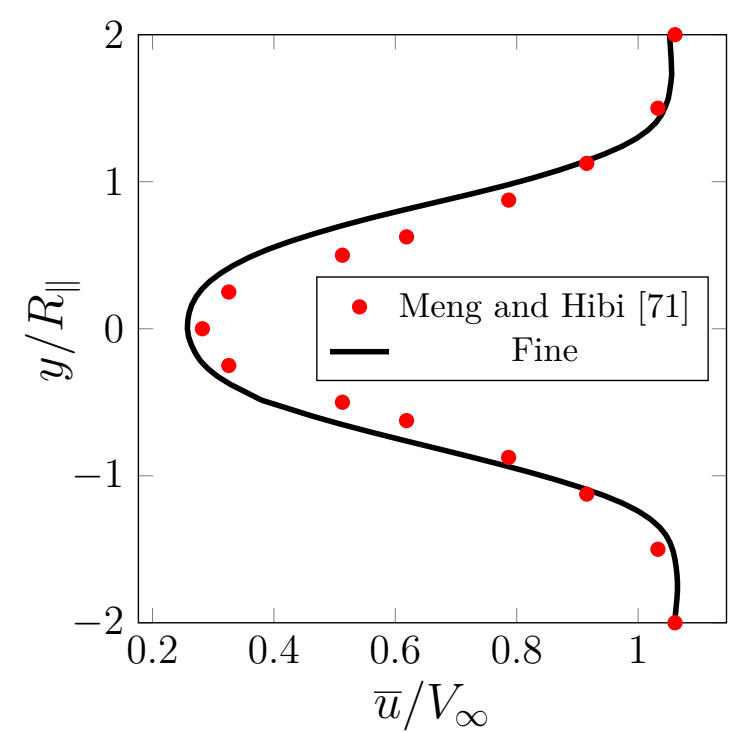

(b) Crosswind measurement line

Figure 28: Experimental vs. numerical time averaged streamwise velocity comparison at a distance of $x / R_{\|}=2.00$ downstream of the building. 
From the vortex visualization of the Q-Criterion, the time averaged velocity profiles, and the experimental comparison, the fine grid density (with 22 cells per building width) is selected for use with the flight simulator and all future database entry CFD simulations. The coarse grid shows the largest variance between the three densities and the expected experimental results. Furthermore, from the LES_IQ it is clear the coarse grid is unacceptable in terms of LES quality. While the required computational time is 5 times more for the fine in comparison to the medium density, they both exhibit similar gradients and minima velocities, the LES_IQ recommends using the fine mesh to resolve at least $80 \%$ of the TKE.

\subsection{RANS Wake Field}

A RANS simulation is performed to reproduce the CFX based CFD simulations of Galway et al. [13] in OpenFOAM, using the suggested grid density and turbulence model. The geometry, domain size, and boundary conditions are the same as the proposed LES, as shown in Figure 16, with the $k-\epsilon$ turbulence closure model. Comparison of the RANS and time averaged LES velocity profiles at $x / R_{\|}=1.25$ downstream of the building, shown in Figure 29, illustrate the close agreement between the simulation methods. The wake field velocities from this RANS simulation is used to evaluate the effects of a RANS versus a LES simulated wake velocities on simulated quadrotor flight, as contained in Chapter 5. 


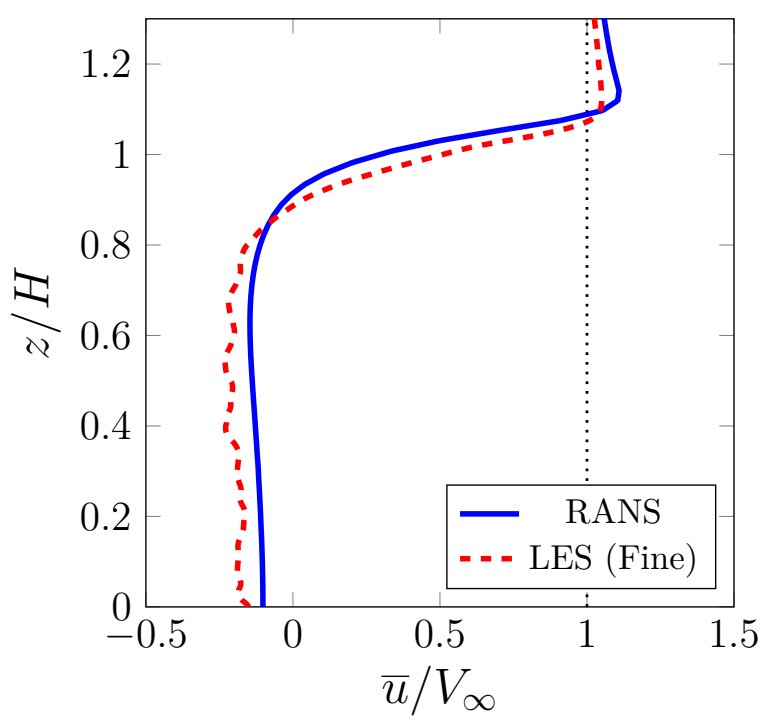

(a) Vertical measurement line

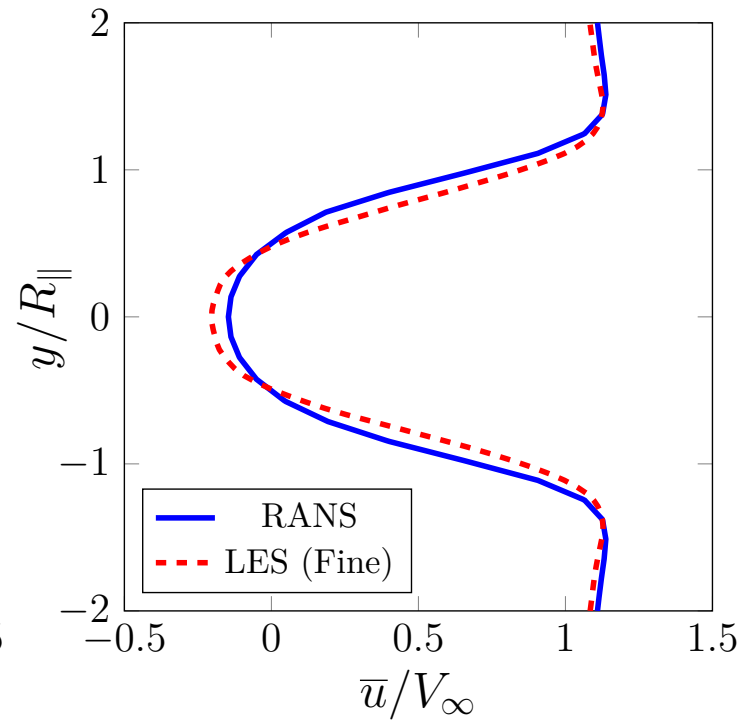

(b) Crosswind measurement line

Figure 29: RANS and time averaged LES streamwise velocity profiles at a distance $x / R_{\|}=1.25$ downstream of the building 


\section{Chapter 4}

\section{Simulation Methodology}

\subsection{Urban Wake Database}

As proposed by the previous work of Galway [13] the use of an urban wake database is two fold. Primarily it separates the dependence on running a CFD simulation in parallel with the flight simulator for the wake velocities. This separation is required due to the different time requirements to run either simulation, to generate the 30 seconds of wake velocities with RANS and LES methods takes approximately 0.97 and 17.7 CPU hours respectively. Furthermore, the database allows for multiple simulated urban building block configurations with only one CFD simulation through matching of the non-dimensional parameters such as the Reynolds number.

Before the simulated wake velocities are uploaded to the database, the results of the CFD simulation are processed through a series of spatial and temporal trimming scripts. The first temporal filtering is achieved through the write interval specified at run time for the CFD simulator, the resolution of this saving interval is detailed 
in Section 4.1.1 below. Next the pressure and velocity components for the entire numerical domain are clipped to a specified region, taken as half a meter inward of each wake refinement boundary to minimize the effect of the refinements on the results. The final step before uploading is an additional temporal trimming in the form of finding an appropriate loop interval for the transient velocity fields given the finite length of simulated CFD time. Finding this loop interval is an iterative process and studies multiple flow results as outlined in the Section 4.1.2. A sample work flow and some database processing scripts are contained in Appendix $\mathrm{C}$

\subsubsection{Temporal Resolution Study}

The grid refinement study in Chapter 3 resulted in the selection of the fine mesh for spatial resolution for the CFD simulations. While the time step for the LES results has been specified such that all spatial and temporal motions are resolved, a resolution study is performed to ascertain an appropriate save interval for the wake fields. Each table in the database represents a time step and each line in a table contains a location in $3 \mathrm{D}$ space and three wake velocity components. For the presented single building case, the CFD simulation is run for over 37,000 time steps and the wake area contains over one and a half million nodes. Therefore, the temporal resolution study is used to find a simulation time step save interval which is fine enough for the flight simulator but minimizes the required database storage disk space and database querying time. 
To find an acceptable time interval the results of a LES saved initially every 0.005 seconds and uploaded to the database. The database is then queried for the streamwise velocity component at the probe location shown in Figure 18 for time steps of $0.005 \mathrm{~s}, 0.01 \mathrm{~s}, 0.05 \mathrm{~s}, 0.1 \mathrm{~s}$, and $0.5 \mathrm{~s}$ (by skipping over the appropriate number of tables in the database). Figure 30 illustrates the returned streamwise velocities for a 1 second sample window.

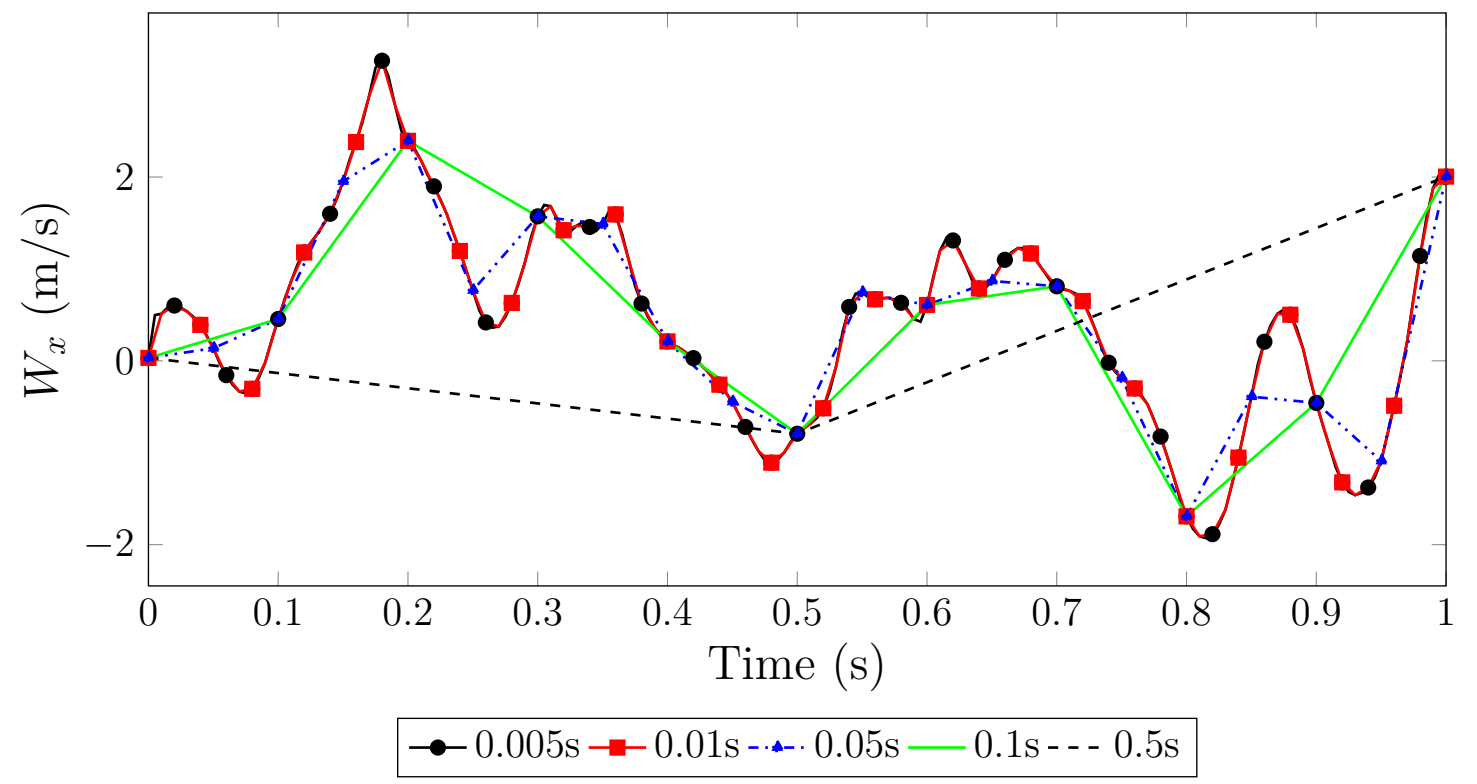

Figure 30: LES wake database timestep resolution study

From Figure 30 it is clear the $0.5 \mathrm{~s}$ resolution is far too coarse to capture an appropriate level of velocity change. Conversely, $0.005 \mathrm{~s}$ and $0.01 \mathrm{~s}$ are nearly indistinguishable making the former unnecessary. To select between the remaining three resolutions the total querying times for the 1 second sampling window are considered, as collected in Table 2 . 
Table 2: Urban wind database query time

\begin{tabular}{cccccc}
\hline \hline & $0.005 \mathrm{~s}$ & $0.01 \mathrm{~s}$ & $0.05 \mathrm{~s}$ & $0.1 \mathrm{~s}$ & $0.5 \mathrm{~s}$ \\
\hline Query Time (s) & 79.6 & 17.6 & 3.8 & 2.8 & 0.6 \\
\hline \hline
\end{tabular}

Since the increase in query time for $0.05 \mathrm{~s}$ is only $36 \%$ more in comparison to 0.1 $\mathrm{s}$ and offers double the resolution, it becomes a choice between $0.01 \mathrm{~s}$ and $0.05 \mathrm{~s}$ second save intervals. While $0.01 \mathrm{~s}$ captures all of the velocity changes (peaks and troughs) it comes at the cost of a $360 \%$ increase in the query time. Considering this substantial increase and the order of the missing resolution with respect to the $1 \mathrm{~s}$ sample window (i.e. 1 second of flight simulation), the $0.05 \mathrm{~s}$ resolution is selected for the database save interval, and used for all future database generation simulations.

\subsubsection{Database Loop Interval}

As previously mentioned the finite end to the CFD results requires a portion of the transient wake field to be sectioned off, saved to the database, and looped over for flight simulations longer than the loop time. The database loop time for both the RANS and LES simulations are determined so that the start and end times reflect a consistent pattern within the flow field. An observation of the entire wake field velocity contours (as shown in Figure 31) is used to establish coarse start and end times.

These times are further refined by examining the side force generated on the building (Figures 32a and 32b) and the crosswind velocity component at a sample point within the wake (Figures 32c and 32d) during the time period identified by the velocity contours in Figure 31. The final start and end times of the 


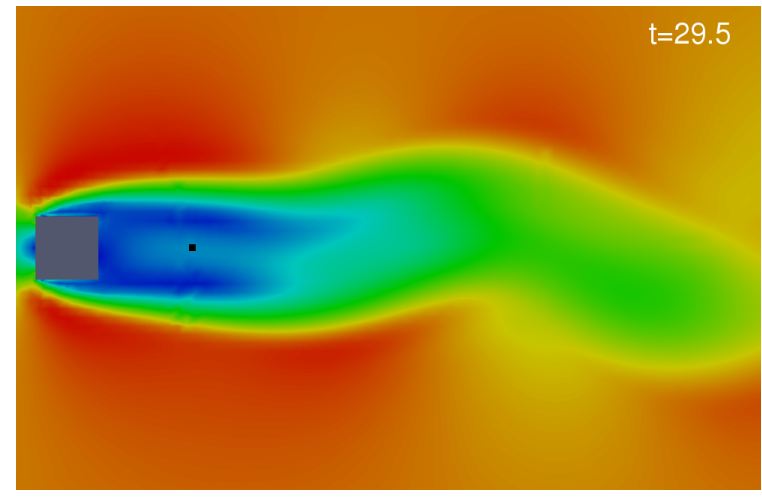

(a) RANS loop start

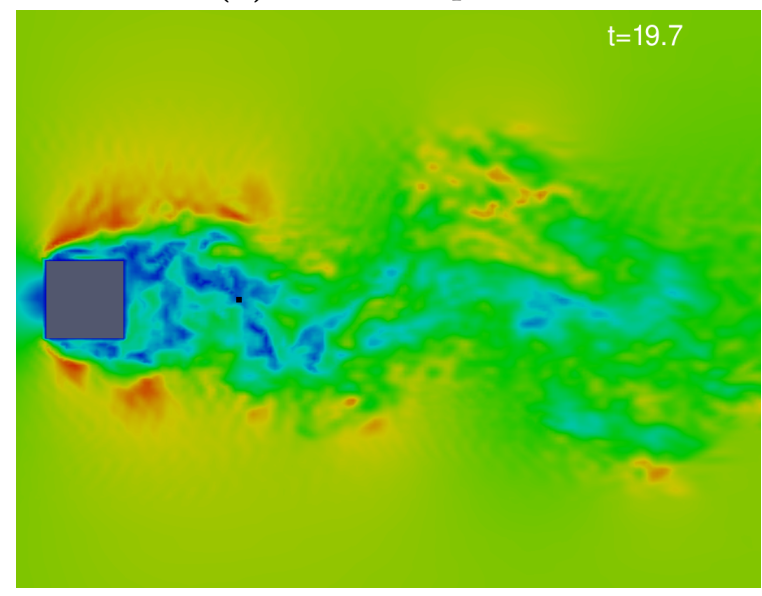

(c) LES loop start

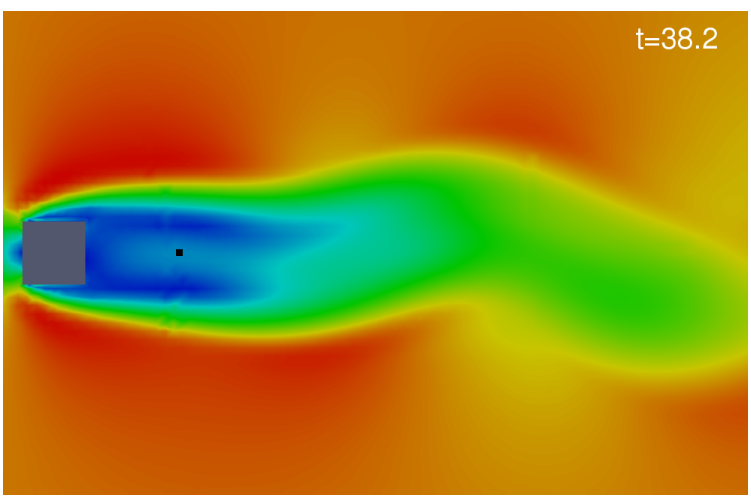

(b) RANS loop end

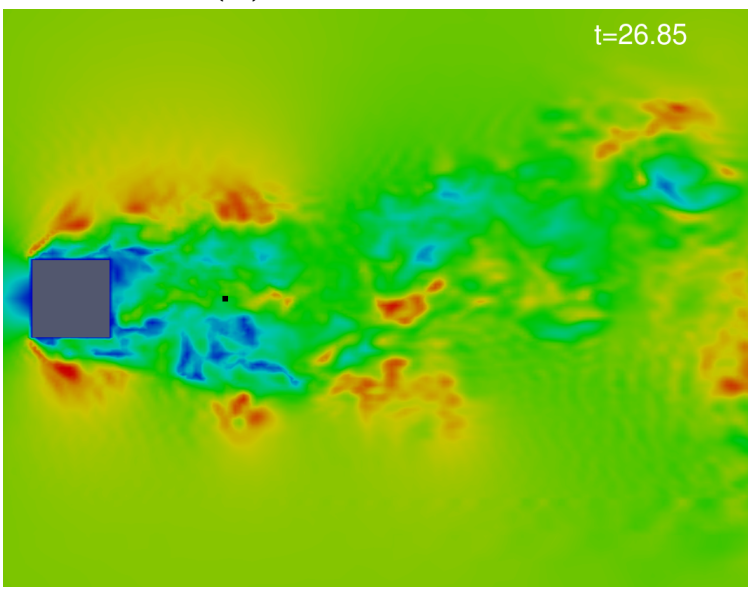

(d) LES loop end

Figure 31: Total velocity contours for visual database loop interval estimation. The small black square illustrates the probe location (Figure 32).

database loop are modified so that crosswind velocity at the wake sample point are approximately equal at the beginning and end of the loop data (thereby minimizing any discontinuous jumps in the wind data). Additionally, the RANS and LES loop times shown in Figure 31 contain approximately 1 flow through time within the refined wake area, which is roughly 8.4 seconds.

The oscillations in both crosswind and building side force resulting from a periodic 


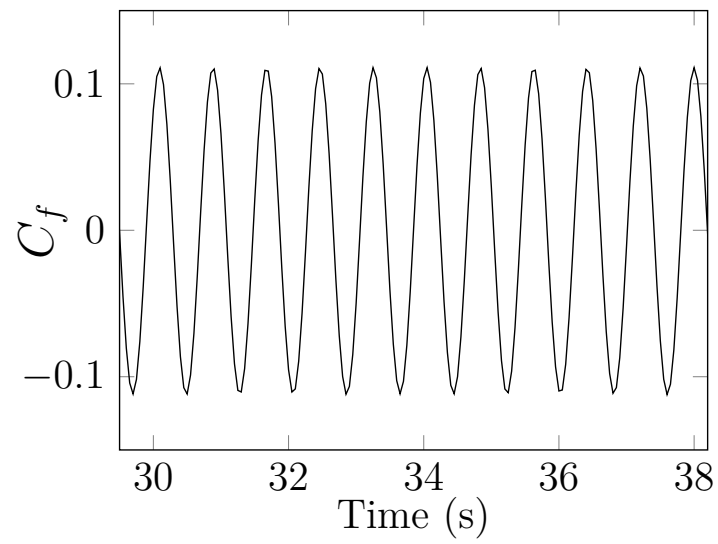

(a) RANS crosswind force coefficient

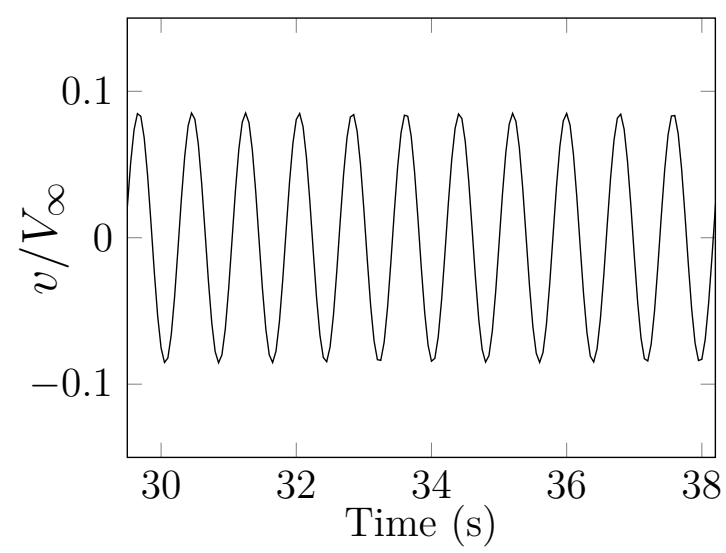

(c) RANS crosswind velocity probed at $x / R_{\|}=2.00, z / H=0.625$.

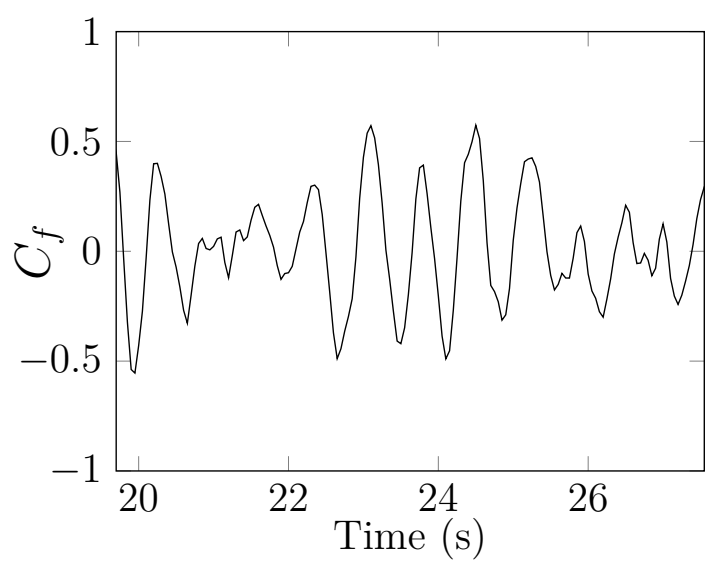

(b) LES crosswind force coefficient

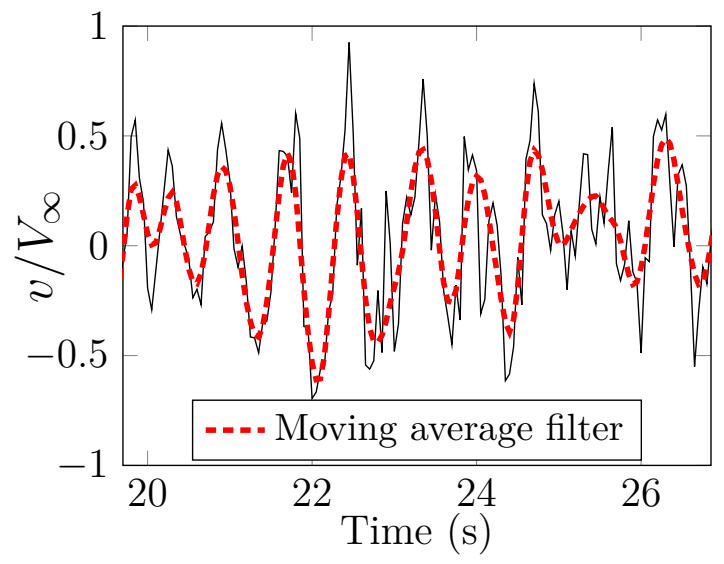

(d) LES crosswind velocity probed at $x / R_{\|}=2.00, z / H=0.625$.

Figure 32: Database loop crosswind force coefficient and probed velocity

shedding of vortices within the building wake are clearly visible in the RANS wind data (see Figure 32a). The LES results in Figure 32b do not show the same clarity of a repeated pattern due to the greater resolution of turbulent structures which are inherently chaotic. However, applying a smoothing moving average filter to the velocity in Figure 32d produces a cleaner pattern with similar frequency to that of the RANS velocity oscillation in Figure 32c. 
Considering the building exhibits periodic vortex shedding similar to that of a square cylinder in crossflow, the shedding frequency is used for validation through the Strouhal number,

$$
S t=\frac{f R_{\perp}}{V}
$$

Where $f$ is the shedding frequency, $R_{\perp}$ is the wind perpendicular building length, and $V$ is the freestream velocity. Based on the Strouhal number from experimental $[79,80]$ and numerical $[81,82]$ studies the shedding frequency is found to be approximately 1.28 Hz. The approximate 9 and 7 seconds RANS and LES loop intervals, shown in Figures 32c and 32d, exhibit frequencies of $1.26 \mathrm{~Hz}$ and $1.34 \mathrm{~Hz}$ respectively.

\section{$4.2 \quad$ Flight Controller}

There are several configurations for a multi-rotor vehicle in regards to the number of motors used. As the name implies a quadrotor is a multi-rotor with four motors arranged in a plus or cross configuration. There is little difference in the performance between the two frame styles. The plus configuration is used in the flight simulator and it allows a more conceptual and clear derivation of a quadrotor's motion.

One of the benefits of a quadrotor is the simplicity of the propulsion system when compared to that of a helicopter, however this propulsion method introduces challenges for vehicle control. A quadrotor has six degrees of freedom, three positions $[x, y, z]$ and three rotations in space $[\psi, \theta, \phi]$. The control challenge arises since a quadrotor can only vary the angular velocity of the four motors and therefore 
results in an underactuated system. This produces a coupling between two of the six degrees of freedom. For the quadrotor the $x$ and $y$ translations are coupled with the pitch $\theta$ and roll $\phi$ angles. Figure 33 illustrates how the pair of motors on a common axis rotate in the same direction and how changing their rotation velocities is used to control the attitude and position of the quadrotor.

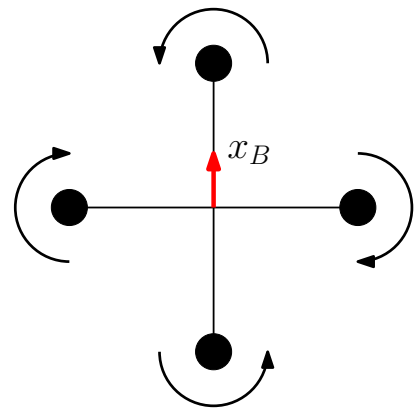

(a) Hover, Ascent, Decent

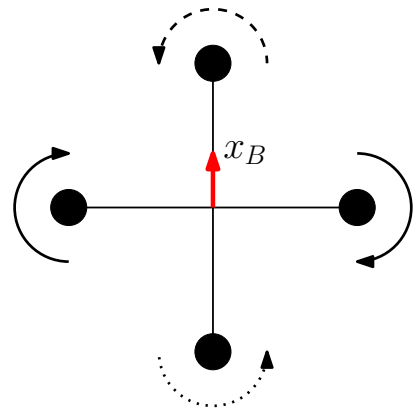

(b) Pitch,Roll

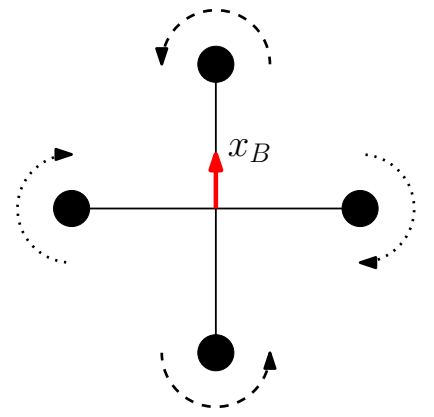

(c) Yaw

Figure 33: Quadrotor, plus configuration, attitude and position control

If all four motor rotations, and thereby thrusts, are equal and assuming no external disturbances, the quadrotor's symmetry will produce a force and moment balance resulting in a hover. To translate only along the z-axis the rotation of all four motors is either uniformly increased or decreased to ascend or descend respectively. To pitch or roll the quadrotor, thereby inducing a x-axis or $\mathrm{y}$-axis translation, the pair of off-axis motors are unchanged while one on-axis motor is increased and the other is decreased. The example in Figure 33b shows the forward motor spinning faster (dashed) while the back motor being reduced (dotted) which results in the quadrotor pitching up and translating along the negative x-axis. The quadrotor's yaw authority is achieved by taking advantage of the motor pair's induced torque on the airframe. By increasing the angular velocity of one pair and decreasing the 
other, the total thrust force is unchanged thereby preventing translation along the z-axis however there is a net torque differential about the z-axis which induces a yaw. The example in Figure 33c shows the counter-clockwise rotating motors are increased (dashed) while the clockwise motors are decreased (dotted). This will cause a positive yaw angle, the quadrotor will rotate in the clockwise direction, as the torque on the body acts in the opposite direction to the motor's rotation.

To develop the equations of motion for the position and attitude of the quadrotor two coordinate systems are used, an inertial Earth fixed frame $\mathcal{F}_{i}$, and a body frame $\mathcal{F}_{b}$. The Earth frame uses a North-East-Down (NED) convention with axis notation of $\mathcal{F}_{i}=\left\{x_{E}, y_{E}, z_{E}\right\}$, where the body frame is fixed at the quadrotor's center of gravity and follows the convention of Etkin [83] such that $\mathcal{F}_{B}=\left\{x_{B}, y_{B}, z_{B}\right\}$ are aligned out the front, right and down respectively. Figure 34 illustrates a simplified quadrotor and the two coordinate frames.

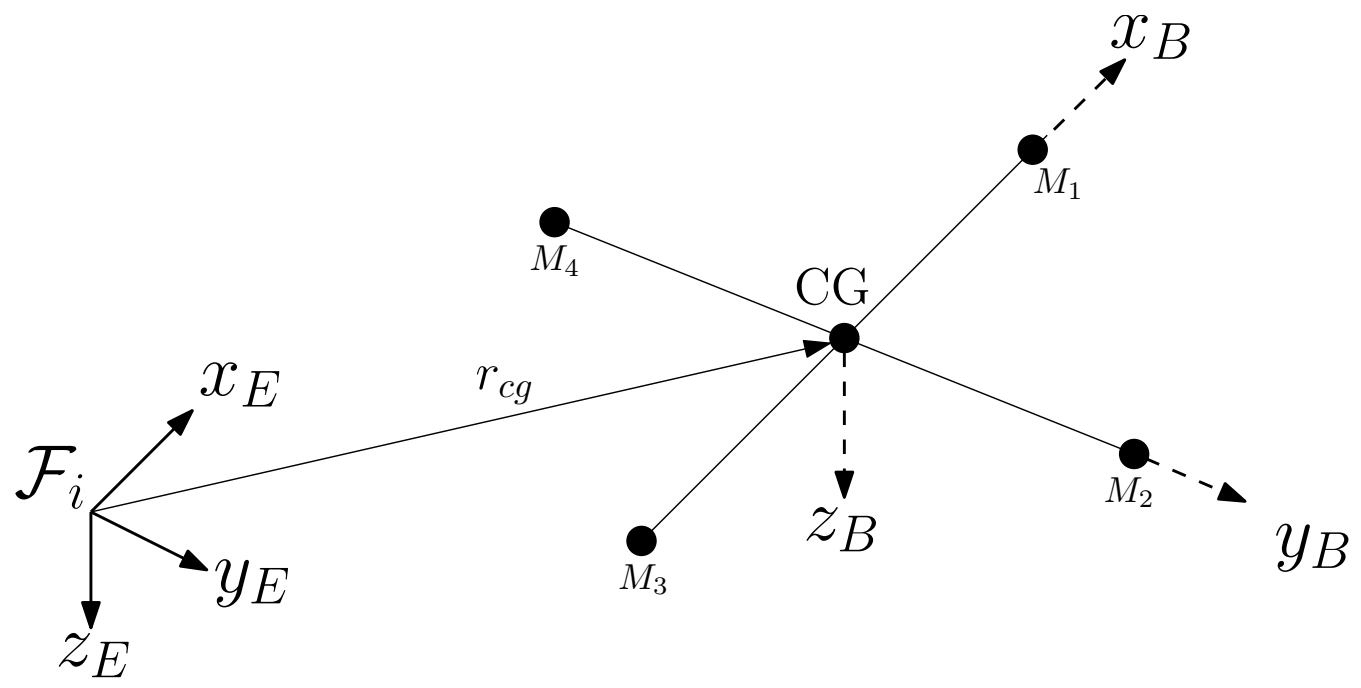

Figure 34: Inertial Earth fixed frame and body quadrotor fixed frame definitions 
A series of three consecutive rotations, Euler angles [83], are used to transform from one frame to the other. The order of these rotations about each axis are important and the yaw, pitch, roll or 3-2-1 order is used here to go from the Earth to the body frame,

$$
L_{B E}=L_{1}(\phi) L_{2}(\theta) L_{3}(\psi)
$$

Where the sequence of the angles is opposite to that of the rotations due to matrix premultiplication [83]. Conversely, to find the body to Earth rotation matrix the reverse rotation sequence is performed,

$$
L_{E B}=L_{3}(-\psi) L_{2}(-\theta) L_{1}(-\phi)
$$

Resulting in the body to Earth rotation matrix,

$$
L_{E B}=\left[\begin{array}{ccc}
\cos \theta \cos \psi & \sin \phi \sin \theta \cos \psi-\cos \phi \sin \psi & \cos \phi \sin \theta \cos \psi+\sin \phi \sin \psi \\
\cos \theta \sin \psi & \sin \phi \sin \theta \sin \psi+\cos \phi \cos \psi & \cos \phi \sin \theta \sin \psi-\sin \phi \cos \psi \\
-\sin \theta & \sin \phi \cos \theta & \cos \phi \cos \theta
\end{array}\right]
$$

Where the angles are limited such that,

$$
\begin{aligned}
&-\pi \leq \phi<\pi \text { or } \quad 0 \leq \psi \leq 2 \pi \\
&-\frac{\pi}{2} \leq \theta \leq \frac{\pi}{2} \\
&-\pi \leq \phi<\pi \quad \text { or } \quad 0 \leq \phi \leq 2 \pi
\end{aligned}
$$

This limiting is due to one of the constraints of Euler angles where the possibility of singularities at certain sets of angles can occur. Focusing on the quadrotor body frame the free body diagram of Figure 35 is generated. 


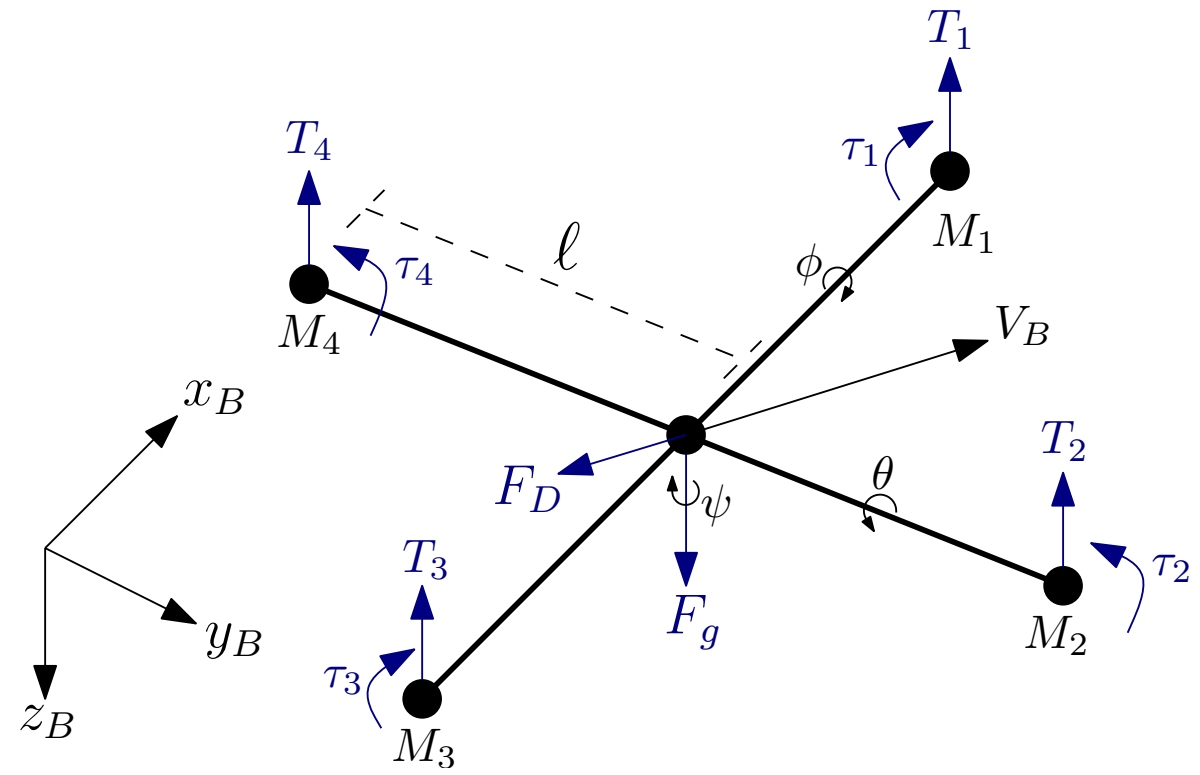

Figure 35: Quadrotor free body diagram and notation convention

Where $M_{i}$ represents the $i$ th motor and $T_{i}$ and $\tau_{i}$ are the thrust and torque of that motor respectively. The formal illustration of the already introduced yaw, pitch and roll angles $(\psi, \theta, \phi)$ are shown in their positive convention. External forces on the quadrotor such as gravity $F_{g}$ and drag $F_{D}$ are shown where the latter acts in the opposite direction to the body frame velocity or airspeed vector $V_{B}$. From this freebody diagram the governing equations of motion are derived starting with the quadrotor as a single rigid body with six degrees of freedom. The force and momentum equations relative to the inertial Earth frame are,

$$
\begin{gathered}
\boldsymbol{F}_{E}=m \dot{\boldsymbol{V}}_{E} \\
\boldsymbol{M}_{E}=\dot{\boldsymbol{h}}_{E}=\frac{d}{d t}\left(\boldsymbol{I}_{E} \cdot \boldsymbol{\omega}\right)
\end{gathered}
$$

where $\boldsymbol{F}_{E}$ is the sum of external forces acting on the quadrotor, $m$ is the quadrotor's mass and $\dot{\boldsymbol{V}}_{E}$ is the quadrotor's acceleration such that $\dot{\boldsymbol{V}}_{E}=\ddot{\boldsymbol{r}}_{c}=\left[\begin{array}{lll}\ddot{X}_{E} & \ddot{Y}_{E} & \ddot{Z}_{E}\end{array}\right]^{T}$. 
$\boldsymbol{M}_{E}$ is the external moment vector about the quadrotor center of gravity, $\dot{\boldsymbol{h}}_{E}$ is the angular momentum, $\boldsymbol{I}_{E}$ is the inertia tensor and $\boldsymbol{\omega}$ is the angular velocity vector. The components of the angular velocity vector are the angular rates of the quadrotor such that,

$$
\boldsymbol{\omega}=\left[\begin{array}{lll}
p & q & r
\end{array}\right]^{T}
$$

and the inertia matrix is defined as,

$$
\boldsymbol{I}_{E}=\left[\begin{array}{ccc}
I_{x x} & -I_{x y} & -I_{x z} \\
-I_{y z} & I_{y y} & -I_{y z} \\
-I_{z x} & -I_{z y} & I_{z z}
\end{array}\right]
$$

While an inertial frame is required for valid application of Equation (52a), it results in a time derivative for the angular momentum where both $\boldsymbol{I}_{E}$ and $\boldsymbol{\omega}$ change with motion and become variables. Therefore the forces and moments are expressed in the body frame by applying the Euler angle rotation matrix $\boldsymbol{L}_{E B}$ defined in Equation (51). Starting with the force equation, extra care is required when using the body frame due to the body fixed velocity vector $\boldsymbol{V}_{B}$. Since this vector by definition rotates about $\mathcal{F}_{B}$, and the origin of $\mathcal{F}_{B}$ can rotate about $\mathcal{F}_{E}$, the relative motion between $\boldsymbol{V}_{B}$ and $\mathcal{F}_{E}$ causes the direction cosines in the rotation matrix to change with time,

$$
\boldsymbol{L}_{E B} \boldsymbol{F}_{B}=m \frac{d}{d t}\left(\boldsymbol{L}_{E B} \boldsymbol{V}_{B}\right)=m\left(\boldsymbol{L}_{E B} \dot{\boldsymbol{V}}_{B}+\dot{\boldsymbol{L}}_{E B} \boldsymbol{V}_{\boldsymbol{B}}\right)
$$

Where $\boldsymbol{V}_{B}=[u, v, w]^{T}$. The last term $\dot{\boldsymbol{L}}_{E B} \boldsymbol{V}_{\boldsymbol{B}}$ is the effect of the relative rotation. From the definition of this derivative of a transformation matrix, outlined in Appendix A.6 of Etkin [83], 


$$
\dot{\boldsymbol{L}}_{E B}=\boldsymbol{L}_{E B} \tilde{\boldsymbol{\omega}}_{B}
$$

Where $\tilde{\boldsymbol{\omega}}_{B}$ is the skew-symmetric matrix of $\boldsymbol{\omega}_{B}$ from the definition of vector multiplication,

$$
\tilde{\boldsymbol{\omega}}_{B}=\left[\begin{array}{ccc}
0 & -r & q \\
r & 0 & -p \\
-q & p & 0
\end{array}\right]
$$

Where $p, q$, and $r$ are the roll, pitch, and yaw rates respectively. After substitution the body frame force equation becomes,

$$
\boldsymbol{F}_{B}=m \dot{\boldsymbol{V}}_{B}+\tilde{\boldsymbol{\omega}}_{B} m \boldsymbol{V}_{B}
$$

From the free body diagram of Figure 35, the external forces on the quadrotor are the motor's thrust, gravity, and airframe drag such that $\boldsymbol{F}_{B}=\boldsymbol{F}_{T_{B}}+\boldsymbol{F}_{g_{B}}+\boldsymbol{F}_{D_{B}}$. Where $\boldsymbol{F}_{T_{B}}$ is the total thrust force from a summation of each motor's individual thrust. It is assumed the motor and propeller plane remains orthogonal to the body frame, i.e. there is no blade flapping or elastic effects, and therefore the motor thrust always acts in the negative $\mathrm{z}$-axis,

$$
\boldsymbol{F}_{T_{B}}=\left[\begin{array}{c}
0 \\
0 \\
-\sum_{i=1}^{4} T_{i}
\end{array}\right]
$$

The gravitational acceleration always acts in the positive z-axis of the inertial Earth frame therefore force is transformed from the Earth to body frame using the rotation 
matrix of Equation (51) such that $\boldsymbol{F}_{g_{B}}=\boldsymbol{L}_{B E} \boldsymbol{F}_{g_{E}}$,

$$
\boldsymbol{F}_{g_{B}}=m g\left[\begin{array}{c}
-\sin \theta \\
\cos \theta \sin \phi \\
\cos \theta \cos \phi
\end{array}\right]
$$

Where $m$ is the quadrotor mass and $g$ gravitational acceleration. To find the body frame drag force due to the to airframe, the body frame velocity vector is resolved into its components and the drag force is calculated for each axis,

$$
\boldsymbol{F}_{D_{B}}=-\frac{\rho}{2}\left[\begin{array}{l}
C_{D_{x}} V_{B}^{2} S_{x} \\
C_{D_{y}} V_{B}^{2} S_{y} \\
C_{D_{z}} V_{B}^{2} S_{z}
\end{array}\right]
$$

Where $\rho$ is the density of the air, $C_{d}$ is the airframe drag coefficient, equal to that of a cube, and $S_{i}$ is the normal airframe surface area. Therefore collecting Equations (58), (59), (60), and (61), results in the system of equations for linear acceleration of the quadrotor in the body frame,

$$
\begin{aligned}
& \ddot{x}_{B}=\dot{u}=r v-q w-g \sin \theta-\frac{F_{D_{x}}}{m} \\
& \ddot{y}_{B}=\dot{v}=p w-r u+g \cos \theta \sin \phi-\frac{F_{D_{y}}}{m} \\
& \ddot{z}_{B}=\dot{w}=q u-p v-\frac{1}{m} \sum_{i=1}^{4} T_{i}+g \cos \theta \cos \phi-\frac{F_{D_{z}}}{m}
\end{aligned}
$$

The next task is to study the moment balance of the quadrotor. Starting with the 
derived Earth frame moment in Equation (52b), the Earth to body rotation matrix is applied. The moment of inertia matrix is now in the body frame and becomes independent of time as the frame is assumed to be rigid resulting in,

$$
\boldsymbol{L}_{E B} \boldsymbol{M}_{B}=\boldsymbol{I}_{B} \frac{d}{d t}\left(\boldsymbol{L}_{E B} \dot{\boldsymbol{\omega}}+\dot{\boldsymbol{L}}_{E B} \boldsymbol{\omega}\right)
$$

Applying the definition of Equation (56) and simplifying the body frame moment equation becomes,

$$
\boldsymbol{M}_{B}=\boldsymbol{I}_{B} \dot{\boldsymbol{\omega}}_{B}+\tilde{\boldsymbol{\omega}}_{B} \boldsymbol{I}_{B} \boldsymbol{\omega}_{B}
$$

Where $\boldsymbol{I}_{B}$ is the moment of inertia matrix for the rigid body quadrotor. The off diagonal terms of the inertia tensor due to imbalances in the mass distribution are negligible,

$$
\boldsymbol{I}_{B}=\left[\begin{array}{ccc}
I_{x x} & 0 & 0 \\
0 & I_{y y} & 0 \\
0 & 0 & I_{z z}
\end{array}\right]
$$

The sum of the moments on the quadrotor are a function of the angular velocity from the motor's thrust and the gyroscopic torque induced on the airframe from the rotating blades such that $M_{B}=M_{T_{B}}+M_{G_{B}}$. However, the moment induced by the gyroscopic effect is negligible as it is an order of magnitude lower than the moment induced by the motor thrust. The moment generated by the $i$ th motor is proportional to the thrust force and arm length between the motor and the center of gravity. It is illustrated in Figures 33 and 35 how each pair of motors contributes to a pitching, rolling or yawing moment. Formally this takes the form of, 


$$
\boldsymbol{M}_{T_{B}}=\left[\begin{array}{c}
\ell\left(T_{1}-T_{3}\right) \\
\ell\left(T_{4}-T_{2}\right) \\
T_{1}+T_{3}-T_{2}-T_{4}
\end{array}\right]
$$

Collecting Equations (64), (65), and (66), results in the system of equations for angular acceleration of the ideal plus configuration quadrotor in the body frame,

$$
\begin{aligned}
\dot{p} & =\frac{1}{I_{x x}}\left[\ell\left(T_{1}-T_{3}\right)+q r\left(I_{y y}-I_{z z}\right)\right] \\
\dot{q} & =\frac{1}{I_{y y}}\left[\ell\left(T_{4}-T_{2}\right)+\operatorname{pr}\left(I_{z z}-I_{x x}\right)\right] \\
\dot{r} & =\frac{1}{I_{z z}}\left[T_{1}+T_{3}-T_{2}-T_{4}+p q\left(I_{x x}-I_{y y}\right)\right]
\end{aligned}
$$

With the quadrotor's dynamic equations defined the kinematics are considered and their equations outlined. By definition kinematics is used to describe the geometrically possible motion of a body without considering the forces and moments causing the motion. In the classic sense, kinematics provides the body velocity, both linear and angular, through the time derivative of the positions.

From the previous force and moment equations and for position control, it is evident a relation to calculate the inertial position and velocity from known body frame variables is required. For angular velocity this is achieved since Euler angles are not constant with time and therefore a relationship between the Euler angle rates and the body frame rates is formed. Using the definition of unit vectors, a rotation matrix for the angular velocity is found, 


$$
\boldsymbol{T}=\left[\begin{array}{ccc}
1 & \sin \phi \tan \theta & \cos \phi \tan \theta \\
0 & \cos \phi & -\sin \phi \\
0 & \sin \phi \sec \theta & \cos \phi \sec \theta
\end{array}\right]
$$

Which produces the angular velocity kinematic equations,

$$
\begin{aligned}
& \dot{\phi}=p+q \sin \phi \tan \theta+r \cos \phi \tan \theta \\
& \dot{\theta}=q \cos \phi-r \sin \phi \\
& \dot{\psi}=q \sin \phi \sec \theta+r \cos \phi \sec \theta
\end{aligned}
$$

The final set of equations required are the linear velocity of the quadrotor in the inertial frame and this is where the effect of the urban wind is incorporated on the quadrotor's flight. The wind velocity vector is rotated into the body frame and it's components are summed with the current body frame velocities to a create a new velocity vector such that,

$$
\begin{gathered}
u=u+W_{x_{B}}=\dot{X}_{B} \\
v=w+W_{y_{B}}=\dot{Y}_{B} \\
w=w+W_{z_{B}}=\dot{Z}_{B}
\end{gathered}
$$

where a positive value of wind results in an increase in the vehicle airspeed (hence the wind velocity is taken positive when it acts along the negative direction of the body frame axes). This updated velocity is then also used to find the quadrotor's linear velocity in the inertial frame by applying the body to Earth rotation matrix Equation (51), 


$$
\begin{aligned}
& \dot{X}_{E}=\dot{X}_{B} \cos \theta \cos \psi+\dot{Y}_{B}(\sin \phi \sin \theta \cos \psi-\cos \phi \sin \psi)+\dot{Z}_{B}(\cos \phi \sin \theta \cos \psi+\sin \phi \sin \psi) \\
& \dot{Y}_{E}=\dot{X}_{B} \cos \theta \sin \psi+\dot{Y}_{B}(\sin \phi \sin \theta \sin \psi+\cos \phi \cos \psi)+\dot{Z}_{B}(\cos \phi \sin \theta \sin \psi-\sin \phi \cos \psi) \\
& \dot{Z}_{E}=-\dot{X}_{B} \sin \theta+\dot{Y}_{B} \sin \phi \cos \theta+\dot{Z}_{B} \cos \phi \cos \theta
\end{aligned}
$$

To close the system of twelve equations quadrotor specific constants and experimentally found values are used, shown below in Table 3. The quadrotor has dimensions of approximately $430 \mathrm{~mm}$ by $430 \mathrm{~mm}$ by $125 \mathrm{~mm}$ and a mass of $2.2 \mathrm{~kg}$. The central structure of the body/fuselage is cubic prism for a simple drag coefficient estimation

\begin{tabular}{|c|c|c|}
\hline Mass & $(m)$ & $2.2 \mathrm{~kg}$ \\
\hline Arm Length & $(\ell)$ & $0.215 \mathrm{~m}$ \\
\hline Gravity & $(g)$ & $9.81 \mathrm{~m} / \mathrm{s}^{2}$ \\
\hline Air Density & $(\rho)$ & $1.225 \mathrm{~kg} / \mathrm{m}^{3}$ \\
\hline \multirow{3}{*}{ Moment of Inertia } & $\left(I_{x x}\right)$ & $1.3894 \times 10^{-2} \mathrm{~kg} \cdot \mathrm{m}^{2}$ \\
\hline & $\left(I_{y y}\right)$ & $1.2621 \times 10^{-2} \mathrm{~kg} \cdot \mathrm{m}^{2}$ \\
\hline & $\left(I_{z z}\right)$ & $2.0518 \times 10^{-2} \mathrm{~kg} \cdot \mathrm{m}^{2}$ \\
\hline \multirow{3}{*}{ Drag Coefficient } & $\left(C_{D_{x}}\right)$ & 1.05 \\
\hline & $\left(C_{D_{y}}\right)$ & 1.05 \\
\hline & $\left(C_{D_{z}}\right)$ & 1.05 \\
\hline \multirow{3}{*}{ Body Area } & $\left(S_{x}\right)$ & $0.0136 \mathrm{~m}^{2}$ \\
\hline & $\left(S_{y}\right)$ & $0.0136 \mathrm{~m}^{2}$ \\
\hline & $\left(S_{z}\right)$ & $0.0256 \mathrm{~m}^{2}$ \\
\hline
\end{tabular}
and convenient construction techniques.

Table 3: Quadrotor flight simulator parameters 
To achieve autonomous attitude and position control a cascaded control scheme using a PID controller for the attitude and PD controller for the position is implemented as illustrated in Figure 36,

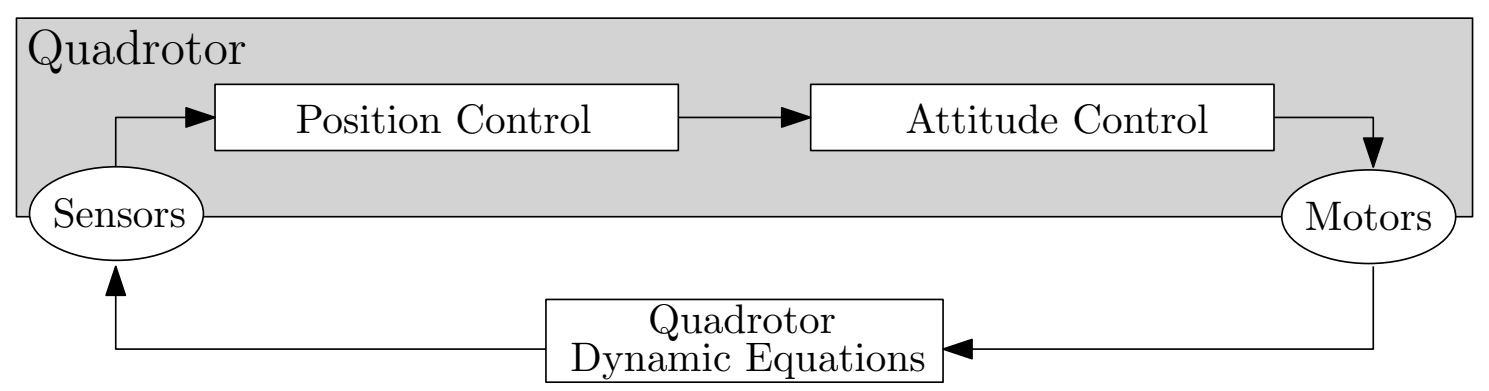

Figure 36: Quadrotor cascade attitude and position control method

Due to the varied number of methods available for a quadrotor to determine its position (GPS, sonar, LIDAR, etc.) no particular sensor error is modelled in that the controller accepts its position directly from the quadrotor dynamics as its true position in space. Based on this position information and the desired location, a commanded attitude is determined using a PD controller,

$$
\begin{gathered}
\theta_{\text {des }}=K_{P_{X}}\left(X_{\text {des }}-X\right)+K_{D_{X}}\left(\dot{X}_{\text {des }}-\dot{X}\right) \\
\phi_{\text {des }}=K_{P_{Y}}\left(Y_{\text {des }}-Y\right)+K_{D_{Y}}\left(\dot{Y}_{\text {des }}-\dot{Y}\right)
\end{gathered}
$$

This desired attitude is then compared to the current attitude as determined from the data fusion of a magnetometer and a 6 -axis inertial measurement unit (with modelled sensor error) from the Simulink Aerospace Blockset. A PID controller is then used to minimize the error between the desired angles from the position controller and the estimated orientation, 


$$
\begin{gathered}
U_{\theta}=K_{P_{\theta}}\left(\theta_{\text {des }}-\theta\right)+K_{D_{\theta}}\left(\dot{\theta}_{\text {des }}-\dot{\theta}\right)+K_{I_{\theta}} \int_{0}^{t}\left(\theta_{\text {des }}-\theta\right) \\
U_{\phi}=K_{P_{\phi}}\left(\phi_{\text {des }}-Z\right)+K_{D_{\phi}}\left(\dot{\phi}_{\text {des }}-\dot{\phi}\right)+K_{I_{\psi}} \int_{0}^{t}\left(\psi_{\text {des }}-\psi\right) \\
U_{\psi}=K_{P_{\psi}}\left(\psi_{\text {des }}-\psi\right)+K_{D_{\psi}}\left(\dot{\psi}_{\text {des }}-\dot{\psi}\right)+K_{I_{\psi}} \int_{0}^{t}\left(\psi_{\text {des }}-\psi\right)
\end{gathered}
$$

The pitch, roll, and yaw control actions $\left(U_{\theta}, U_{\phi}, U_{\phi}\right)$ are then split among the appropriate motors according to,

$$
\begin{aligned}
& P W M_{1}=U_{\theta}-U_{\psi} \\
& P W M_{2}=-U_{\phi}+U_{\psi} \\
& P W M_{3}=-U_{\theta}-U_{\psi} \\
& P W M_{4}=U_{\phi}+U_{\psi}
\end{aligned}
$$

This results in a motor specific pulse width modulation (PWM) signal which is converted into a $T_{i}$ value using an experimentally determined relationship between the PWM signal and force generated by an APC 10x4.7 SF propeller and Great Planes Rimfire 400 28-30-950 out-runner motor. The gains for each of the constants shown in the two control schemes are listed in Tables 4 and 5 and remain constant for all the simulation results presented.

Table 4: Position controller PD gains

\begin{tabular}{c|cc|cc|cc}
\hline \hline Gains: & $K_{P_{X}}$ & $K_{D_{X}}$ & $K_{P_{Y}}$ & $K_{D_{Y}}$ & $K_{P_{Z}}$ & $K_{D_{Z}}$ \\
\hline Value: & 0.2 & 4.0 & 0.2 & 4.0 & 0.4 & 1.0 \\
\hline \hline
\end{tabular}

Table 5: Attitude controller PID gains

\begin{tabular}{c|ccc|ccc|ccc}
\hline \hline Gains: & $K_{P_{\phi}}$ & $K_{D_{\phi}}$ & $K_{I_{\phi}}$ & $K_{P_{\theta}}$ & $K_{D_{\theta}}$ & $K_{I_{\theta}}$ & $K_{P_{\psi}}$ & $K_{D_{\psi}}$ & $K_{I_{\psi}}$ \\
\hline Value: & 11.0 & -20 & 0.02 & 11.0 & -20 & 0.02 & 11.0 & 15 & 0.5 \\
\hline \hline
\end{tabular}


A simplified visual of the preceding control loops is shown in Figure 37. On the left side are the quadrotor's state in inertial space and the desired inertial space location (and yaw angle). After the control signal is cascaded through the controllers the resulting control actions are split and summed for the four motors. On a quadrotor an electronic speed controller (ESC) converts this signal to a desired motor RPM, producing a certain amount of thrust. The simulator directly uses an experimentally found relationship between the PWM signal and the amount of thrust force, thereby closing the loop between the governing equations of motion and simulating autonomous position control.

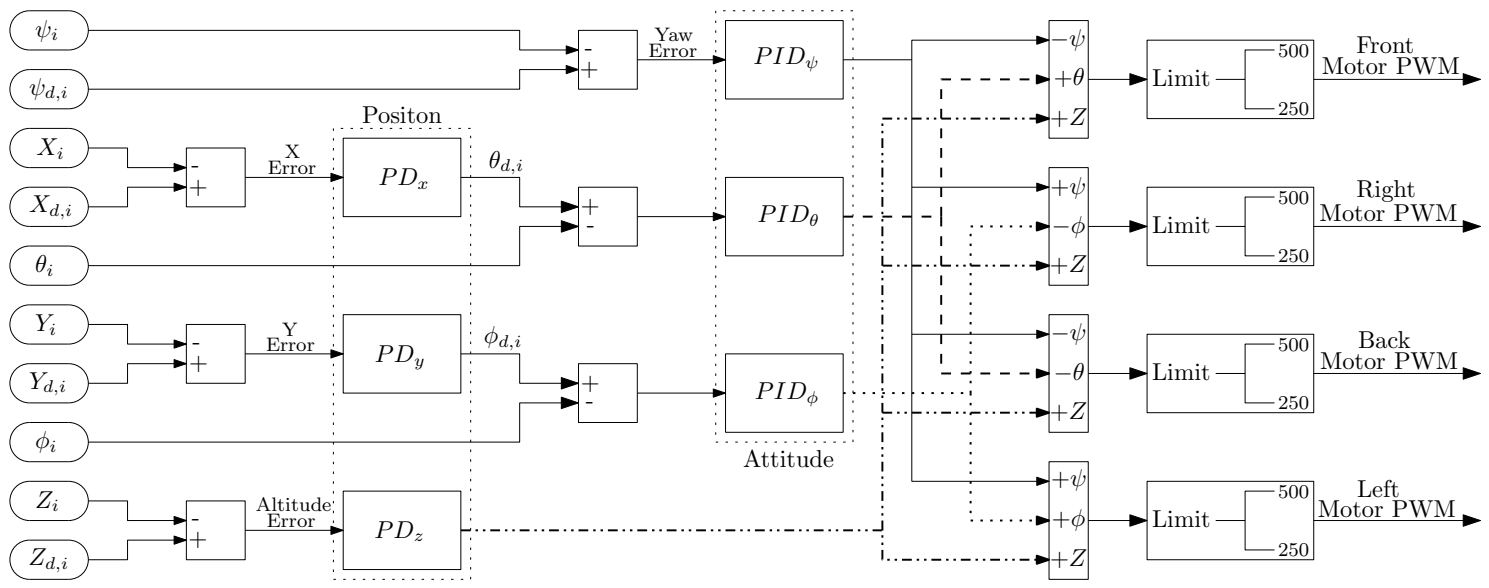

Figure 37: Quadrotor cascade PD-PID control block diagram 


\section{Chapter 5}

\section{Results}

To evaluate the effects of a RANS versus a LES simulated wake field five flight missions are performed. Three of the missions are to hold position at specified locations. Mission 1 is in the undisturbed freestream ahead of the building to establish a vehicle reference performance. Mission 2 is fully within the building wake and represents a location that contains significant regions of recirculating flow. Mission 3 is located along the wake boundary near the building rooftop, where deviations from this position will cause the vehicle to experience significantly varying wind patterns as a mix of the freestream and wake flow conditions. Mission 4 is a vertical ascent through the building wake with a commanded velocity of approximately $1 \mathrm{~m} / \mathrm{s}$, starting five meters off the ground in the building wake and ending in the accelerated velocity region above the roof. Finally the last mission is a horizontal translation along the $y_{E}$ from freestream conditions, through the building wake, and back out to freestream flow. All these missions are shown in the $x_{E^{-}} y_{E}$ plane (Figure 38) and $x_{E^{-}} z_{E}$ plane (Figure 39) superimposed on the RANS and LES velocity field vectors. 


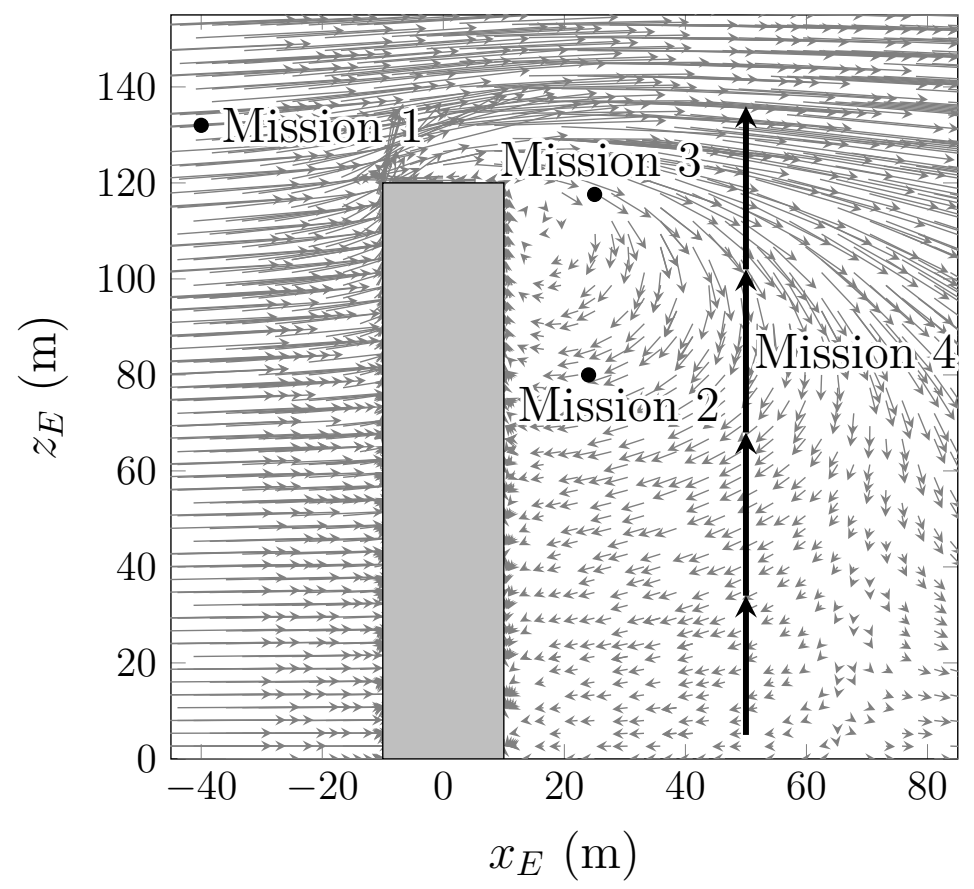

(a) RANS wake field at $\mathrm{t}=38.20 \mathrm{~s}$

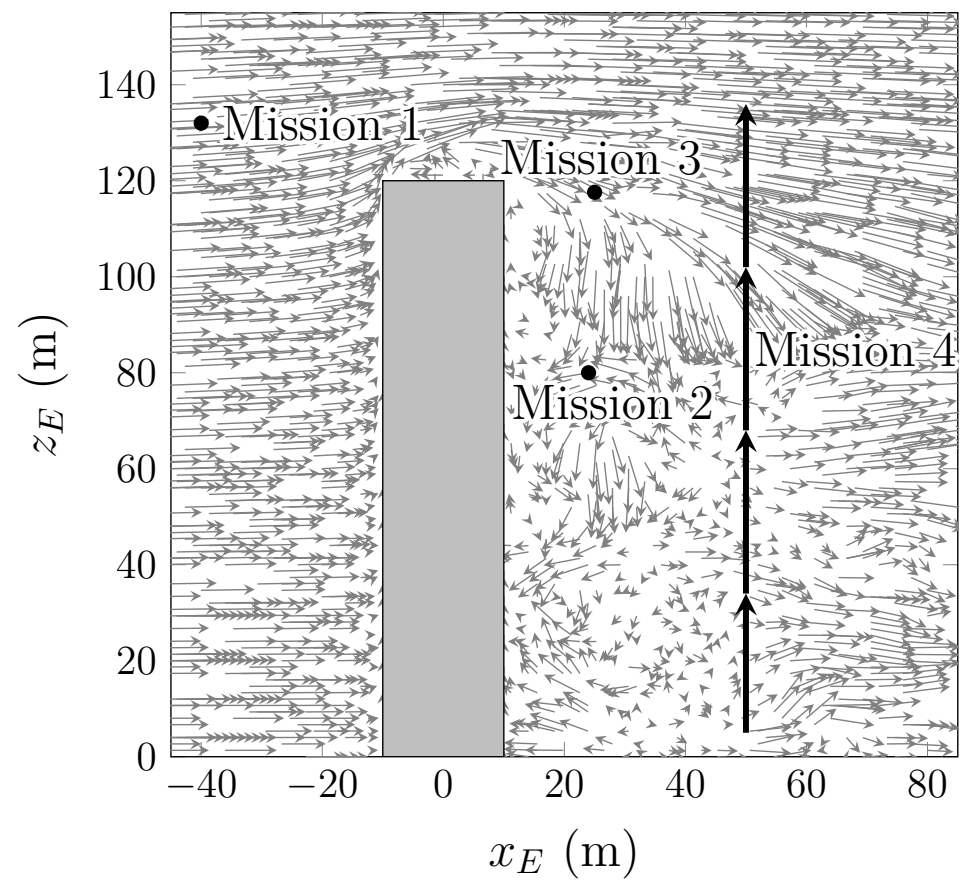

(b) LES wake field at $\mathrm{t}=26.85 \mathrm{~s}$

Figure 38: Flight mission locations and velocity field samples. The RANS vectors are scaled 2.2 times larger than the LES vectors for illustration. 


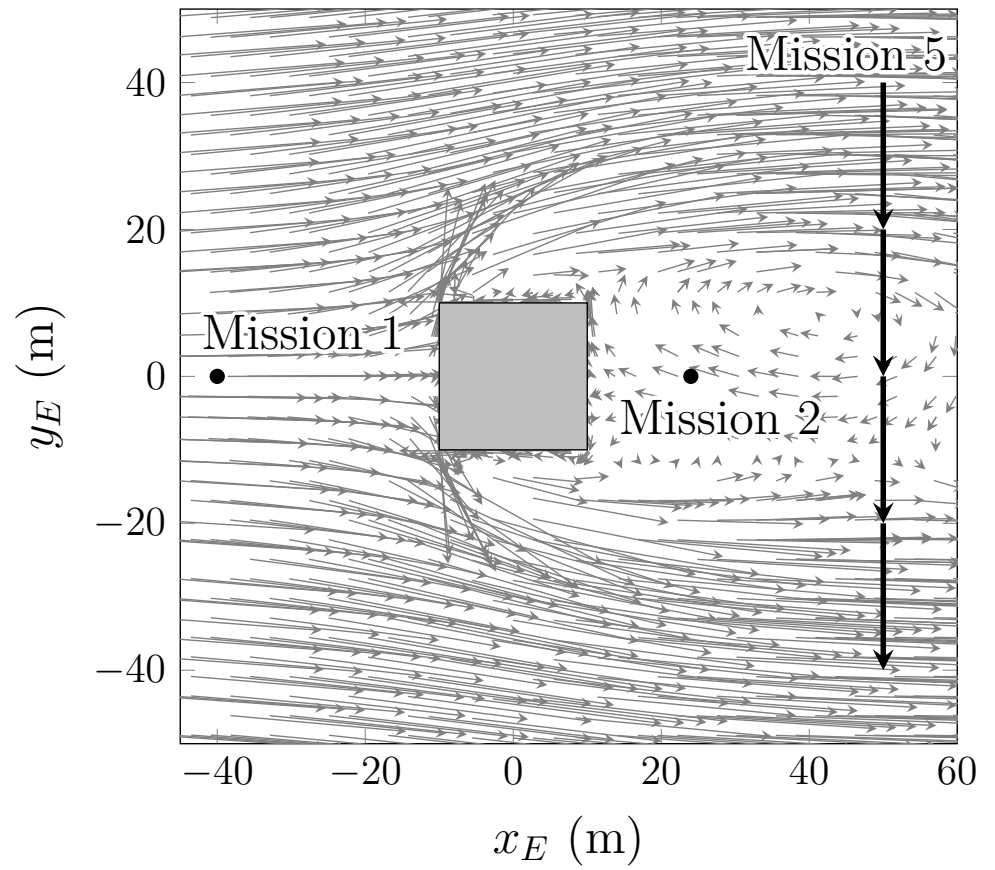

(a) RANS wake field at $\mathrm{t}=38.20 \mathrm{~s}$

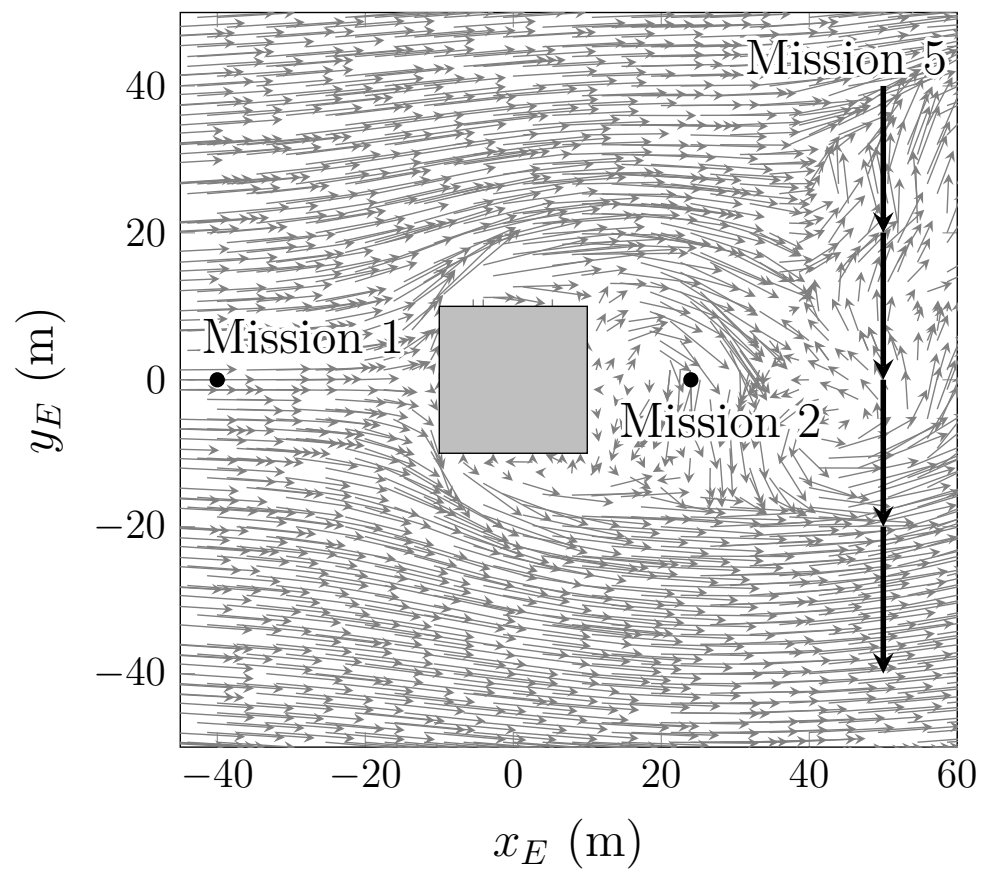

(b) LES wake field at $\mathrm{t}=26.85 \mathrm{~s}$

Figure 39: Flight mission locations and velocity field samples. The RANS vectors are scaled 2.2 times larger than the LES vectors for illustration. 
From the vectors in Figures 38 and 39, not only does LES produce velocities, on average, 2 times higher than RANS, but the resulting wake field is more random. It is evident Mission 2 should have to handle not only the turbulent motions in the wake but the movement of the bulk flow coming back down over the building roof and horseshoe vortices around the side. The crosswind motions are not as prevalent in Mission 3 at roof height, however as shown by the velocity vectors Mission 3 should experience streamwise velocities higher than freestream values as the flow accelerates over the building roof.

Mission 4 should experience a range of changing velocity components as the quadrotor ascends from the wake with all three components changing rapidly (with small magnitudes compared to the freestream) to very directional flow in the accelerated and freestream areas. Mission 5 should experience a similar change as Mission 4 but in the streamwise and crosswind directions as the quadrotor goes from the freestream, through the oscillating wake, and back into the freestream. 


\subsection{Mission 1 - Freestream Wind Position Hold}

Mission 1 is in the freestream wind, outside of any wake affects, to compare the flight performance between: (i) a constant specified value, (ii) RANS wake field, (iii) LES wake field background wind conditions. The velocity components for the three background wind conditions are shown in Figure 40, where it is seen both RANS and LES produces a wind parallel velocity appropriately $6 \%$ less than the 4 $\mathrm{m} / \mathrm{s}$ freestream due to the missions proximity to the building and energy draining effects the turbulence modeling introduces. The sharp increase in velocity from 0 $\mathrm{m} / \mathrm{s}$ to $4 \mathrm{~m} / \mathrm{s}$ between 5 seconds and 10 seconds is a wind ramp unintentionally simulating a large wind gust. This is done to give the controller a 5 second window to initialize and stabilize the quadrotor without any wind influence. The wind is then quickly ramped up to its full value over the next 5 seconds, which from various tests seemed to provide more realistic results in comparison to longer, shallower ramps. Furthermore, there are very small oscillations in the crosswind direction and a small positive normal velocity component due to the wind starting to accelerate over the building roof.

The quadrotor's position over a 125 second hover at the desired location for Mission 1 is shown in Figure 41, separated into the three axis components. The small drop in altitude at the start of the simulation is due to the controller and motor's initializing to place the quadrotor in a hover. The sharp increase in the streamwise direction $X_{E}$ is due to the wind ramp blowing the quadrotor downstream towards the building. As expected all three background wake fields produce similar position deviations of $\pm 0.5 \mathrm{~m}$ from the setpoint. 

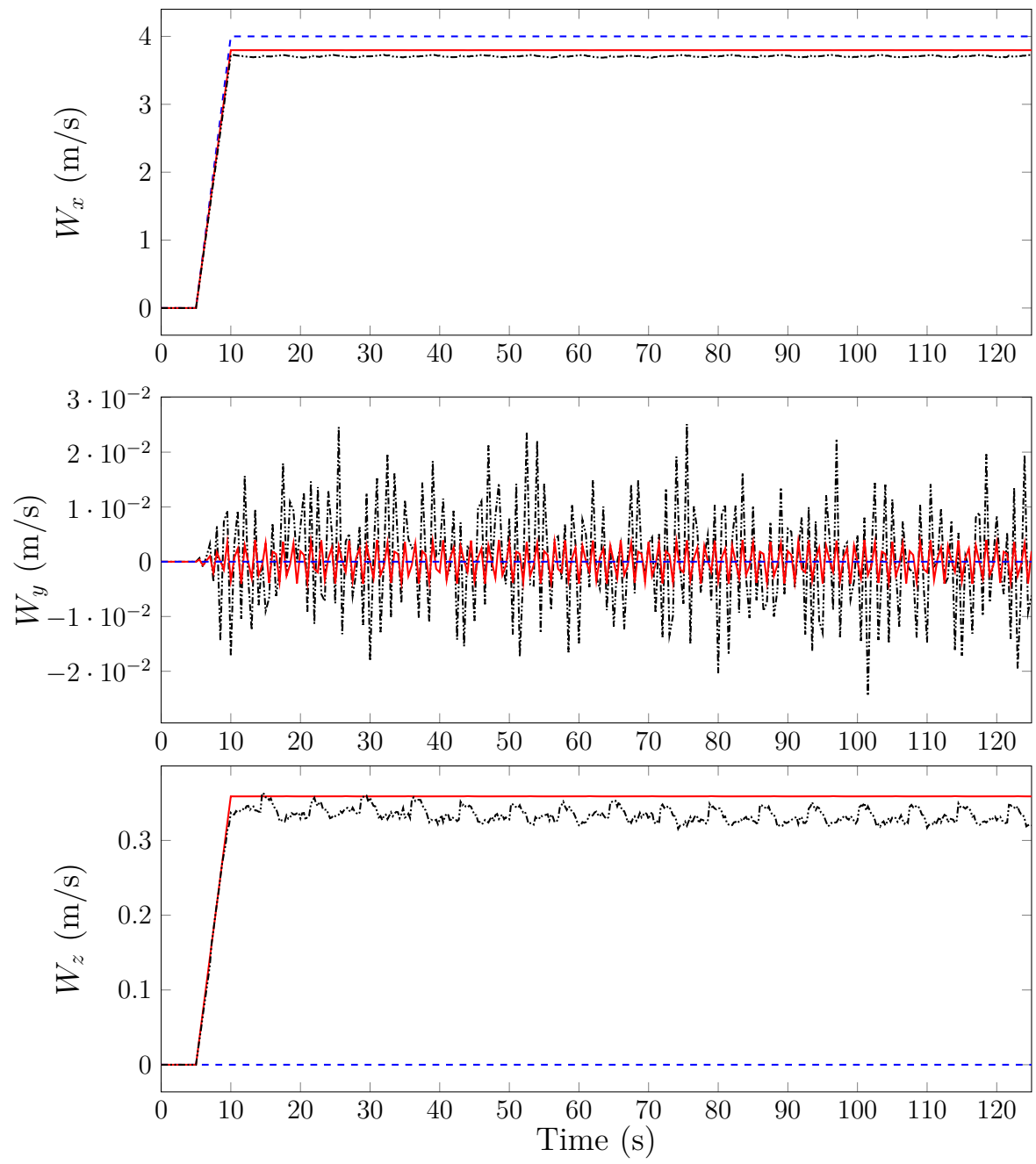

\section{-- - Constant - RANS -.-..... LES}

Figure 40: Mission 1 - wind velocity components

To further illustrate and quantify the position hold flight performance, deviation cuboids are formed representing the maximum quadrotor deviations from the setpoint. Figure 42 shows the bounds within which the quadrotor is able to hold its position for the 125 second flight duration, where the length of the shown coordinate vectors represents a quadrotor body length along that axis. As can be seen, all three 

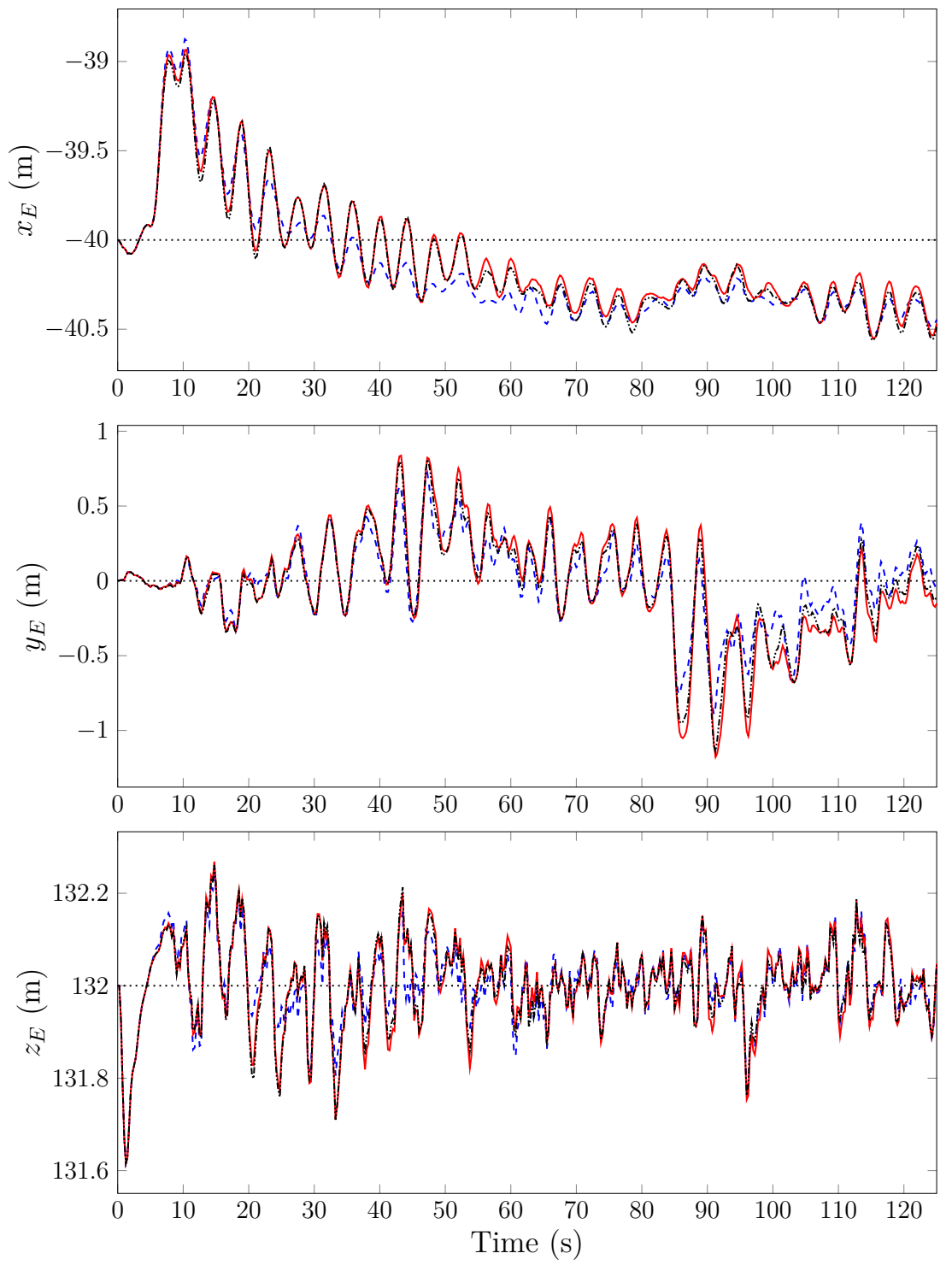

Setpoint --- Constant — RANS -..... LES

Figure 41: Mission 1 - quadrotor position

boxes are of comparable size illustrating the expected result that when flying in a freestream wind condition the simplification of a constant background is reasonably accurate. 


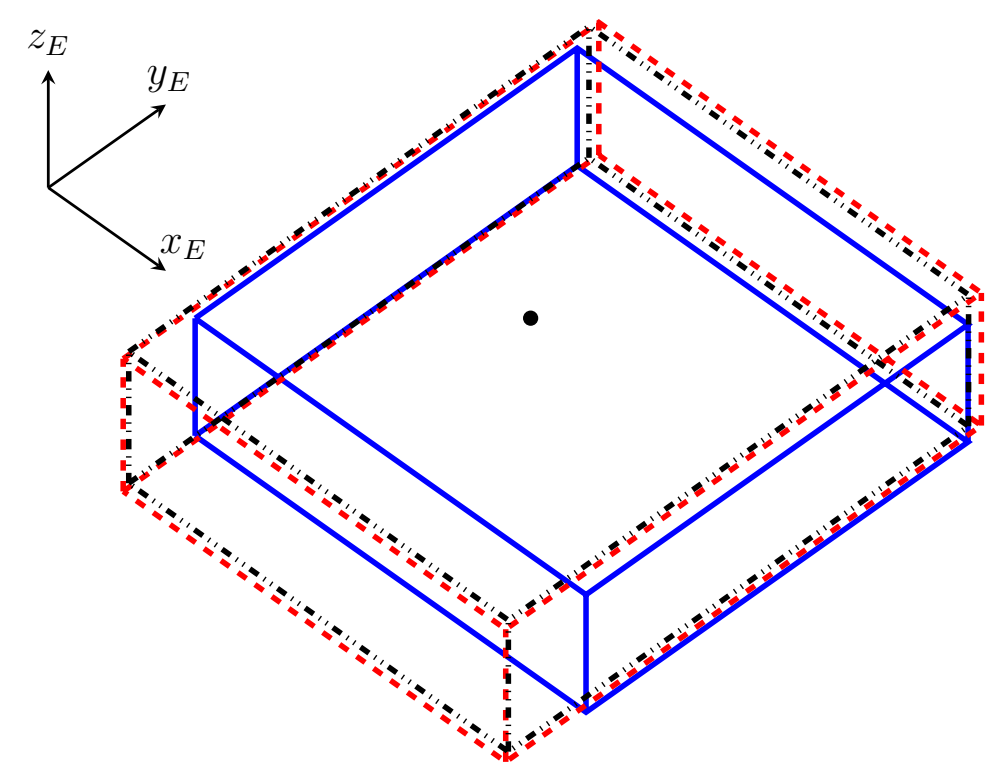

•Setpoint — Constant --- RANS -.- LES

Figure 42: Mission 1 - maximum path deviation bounding boxes. The length of the shown coordinate axis represents a quadrotor body length along that axis.

The use of a RANS simulation increases the deviation volume by approximately $37 \%$ from the deviation volume observed when only a constant wind velocity is assumed, while the LES wind database increases this volume by approximately $42 \%$. The larger increase in the LES bounding box volume is expected in that even though the position of mission one is upstream of the building wake, by virtue of the manner in which the LES upstream boundary conditions are applied, a greater degree of freestream turbulence is present. With a constant background wind the controller is able to hold the quadrotor to within $+2 /-1, \pm 1.25$, and \pm 0.5 body lengths in the $x_{E}, y_{E}$, and $z_{E}$ directions receptively of the desired location. This illustrates the best position hold scenario with a constant but significant wind force in combination with the simulated inertial measurement unit (IMU) noise. 


\subsection{Mission 2 - Building Wake Position Hold}

Given that Mission 2 is located fully within the wake region, the temporal and spatial turbulent motions continually demand corrections from the quadrotor's controller. Fluctuations are generated by multiple vortices which begin along the sides and the roof of the building, get shed downstream, and meet within the wake. However, only the LES wake field resolves these large scale vortices while the RANS wind database averages the fluctuations as shown by the wake velocity components in Figure 43. The RANS components are smoothed from the averaging and have an overall smaller magnitude than the LES wake field velocities.

This variation in wake velocities results in a significant difference in the ability of the quadrotor to hold its position as can be seen in Figure 44. The application of the LES wake velocities generates a substantial decrease in the controllers ability to hold the quadrotor's position. At this location there is a $0.8 \mathrm{~m} / \mathrm{s}$ and $1.0 \mathrm{~m} / \mathrm{s}$ bias in the average velocity acting in the negative $x_{E}$ and $z_{E}$ directions respectively (see Figure 43) which causes the large position deviations seen in Figure 44.

From the position plots of Figure 44 it can be seen how the quadrotor is initially pushed upstream towards the building and slowly returns to a location downstream of the setpoint. Since there is a local wind bias in the negative $x_{E}$ direction (towards the building) this steady state offset is produced to the lack of an integral term in PD position controller (similarity seen in Figures 41 and 47).

Generating the maximum bounding cubiods from the position components clearly 

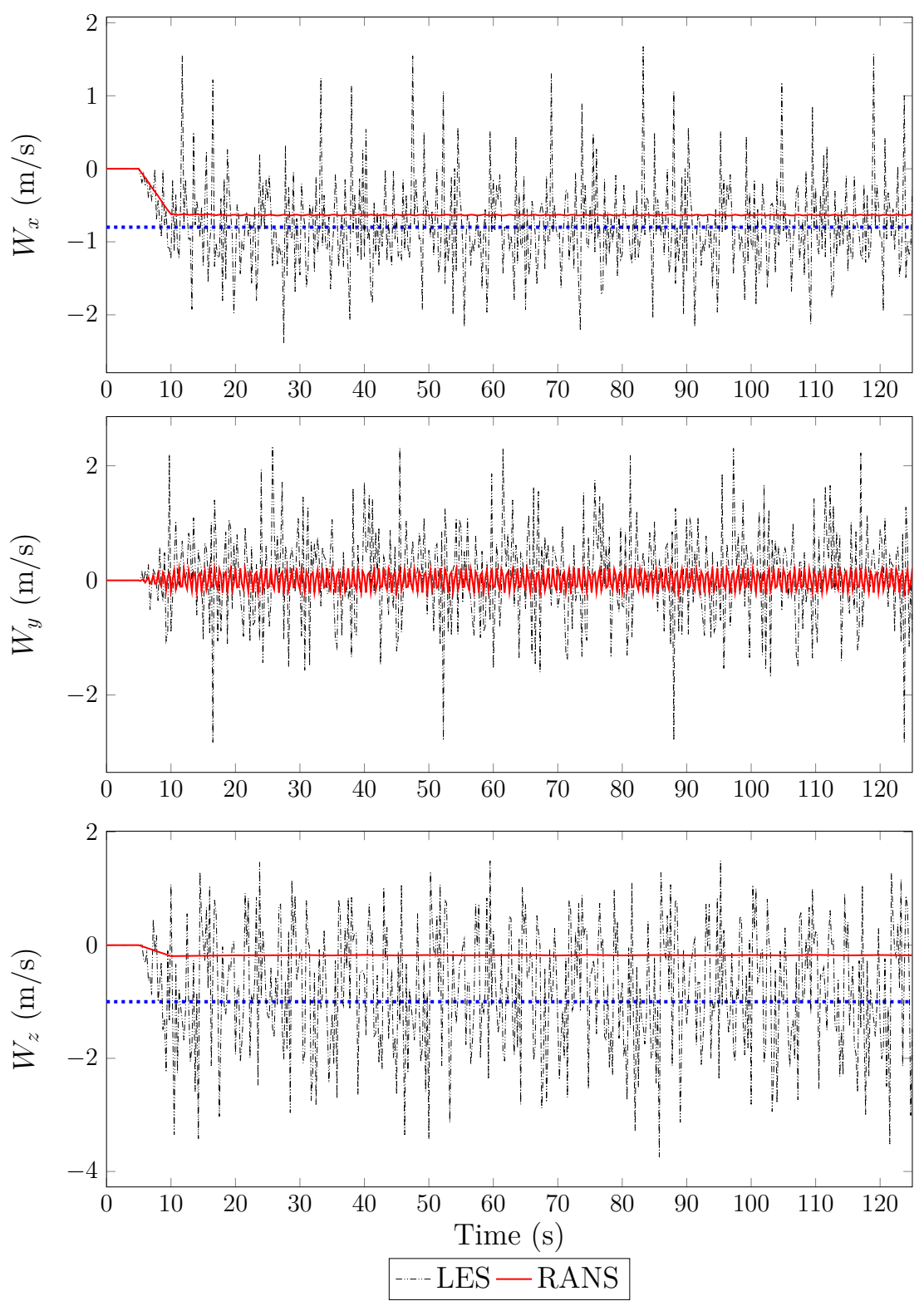

Figure 43: Mission 2 - wind velocity components

shows the resulting increase in setpoint deviation from the LES background wind, shown in Figure 45. The RANS results show a relatively small region within which the same controller is able to hold the quadrotor. Due to the small time averaged 

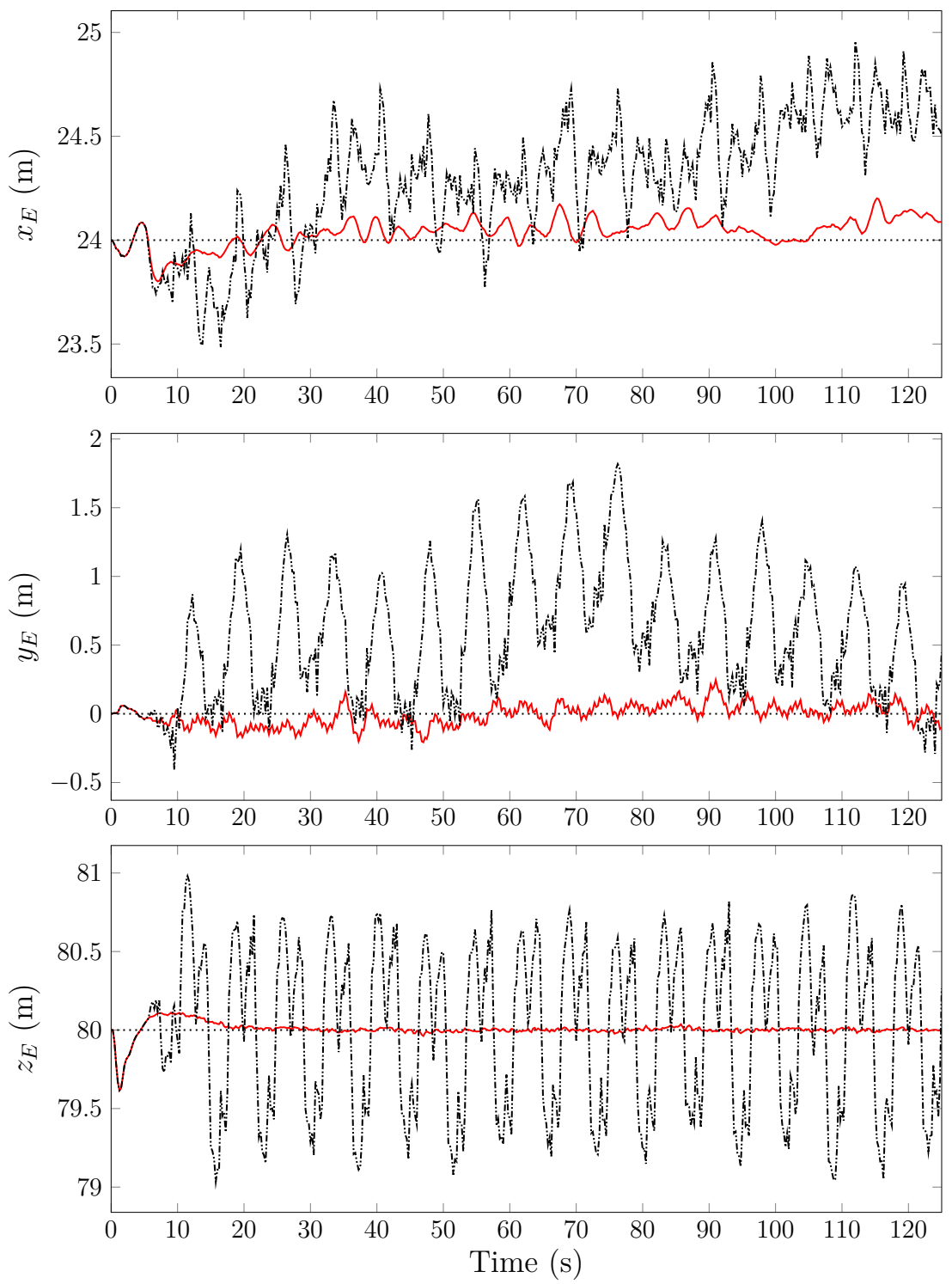

$\cdots$.... Setpoint - RANS - -.... LES

Figure 44: Mission 2 - quadrotor position

velocity in this region the controller is able to hold the quadrotor within a bounding box with a volume of approximately $0.03 \mathrm{~m}^{3}$. This compares to a volume of $7.2 \mathrm{~m}^{3}$ (over a two hundred fold increase) for the same vehicle/controller configuration but 
with the wake field velocities now being supplied from the LES database. If the wake field is calculated using a RANS simulation the predicted performance is that the quadrotor can be held to within \pm 0.5 body lengths in all directions at this location. This is in contrast to when the wake field is obtained from the LES database where then the simulations predict both a loss in isotropy (in that the deviations are not evenly distributed about the setpoint as shown with the relative setpoint locations on the edges of the bounding boxes) and that the absolute value of the deviations are larger. The LES simulations predict a variation of $+2 /-1,+1.5 /-0.5$, and \pm 2 body lengths in the $x_{E}, y_{E}$ and $z_{E}$ directions respectively.

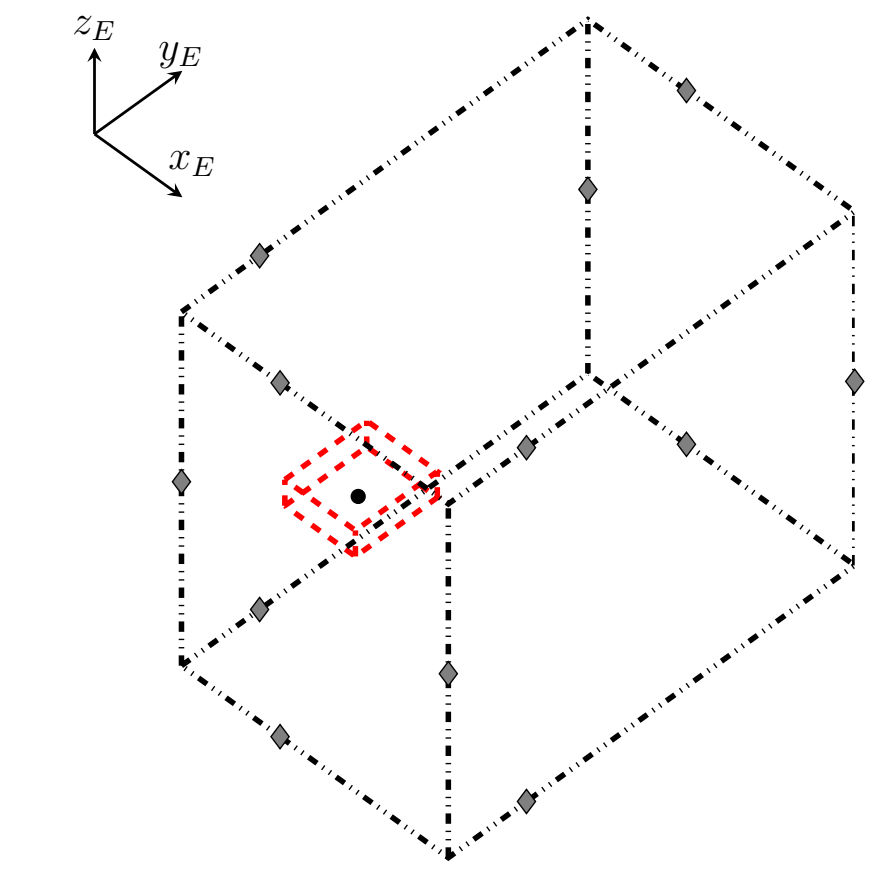

$\bullet$ Setpoint --- RANS -..- LES $\diamond$ Relative Setpoint

Figure 45: Mission 2 - maximum path deviation bounding boxes. The length of the shown coordinate axis represents a quadrotor body length along that axis. 


\subsection{Mission 3 - Top Wake Boundary Position Hold}

Mission 3 is located near the top wake boundary, in the gradient area studied in Figure 21a. The RANS wake field has velocities of approximately $1 \mathrm{~m} / \mathrm{s}$, small oscillations about $0 \mathrm{~m} / \mathrm{s}$, and approximately $-1 \mathrm{~m} / \mathrm{s}$ in the $x_{E}, y_{E}$, and $z_{E}$ directions respectively (Figure 46). The LES wake field velocities have double the average magnitude of the RANS wake field and have average fluctuations of $\pm 1 \mathrm{~m} / \mathrm{s}$ on all three components, also shown in Figure 46.
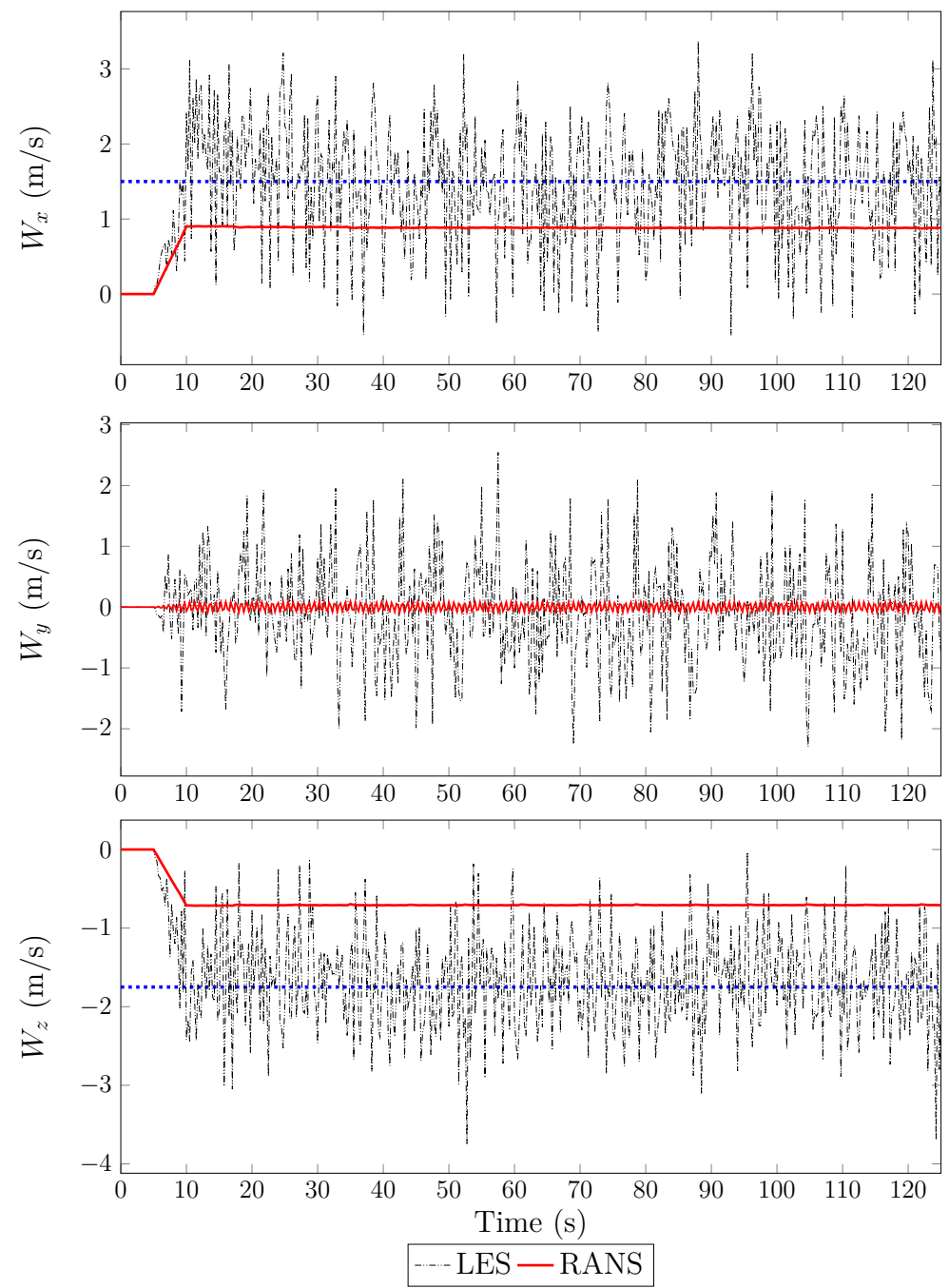

Figure 46: Mission 3 - wind velocity components 
Similar to Mission 2 in the wake, the LES wake field produces much larger position deviations as illustrated in Figure 47. Additionally, the strong wind in the $x_{E}$ direction results in a steady state offset upstream of the setpoint previously seen in Figures 41 and 44 . While the altitude controller is able to maintain $\pm 0.5 \mathrm{~m}$ about the setpoint it comes at the cost of reduced $y_{E}$ axis performace due to the quadrotor's dynamics coupling.
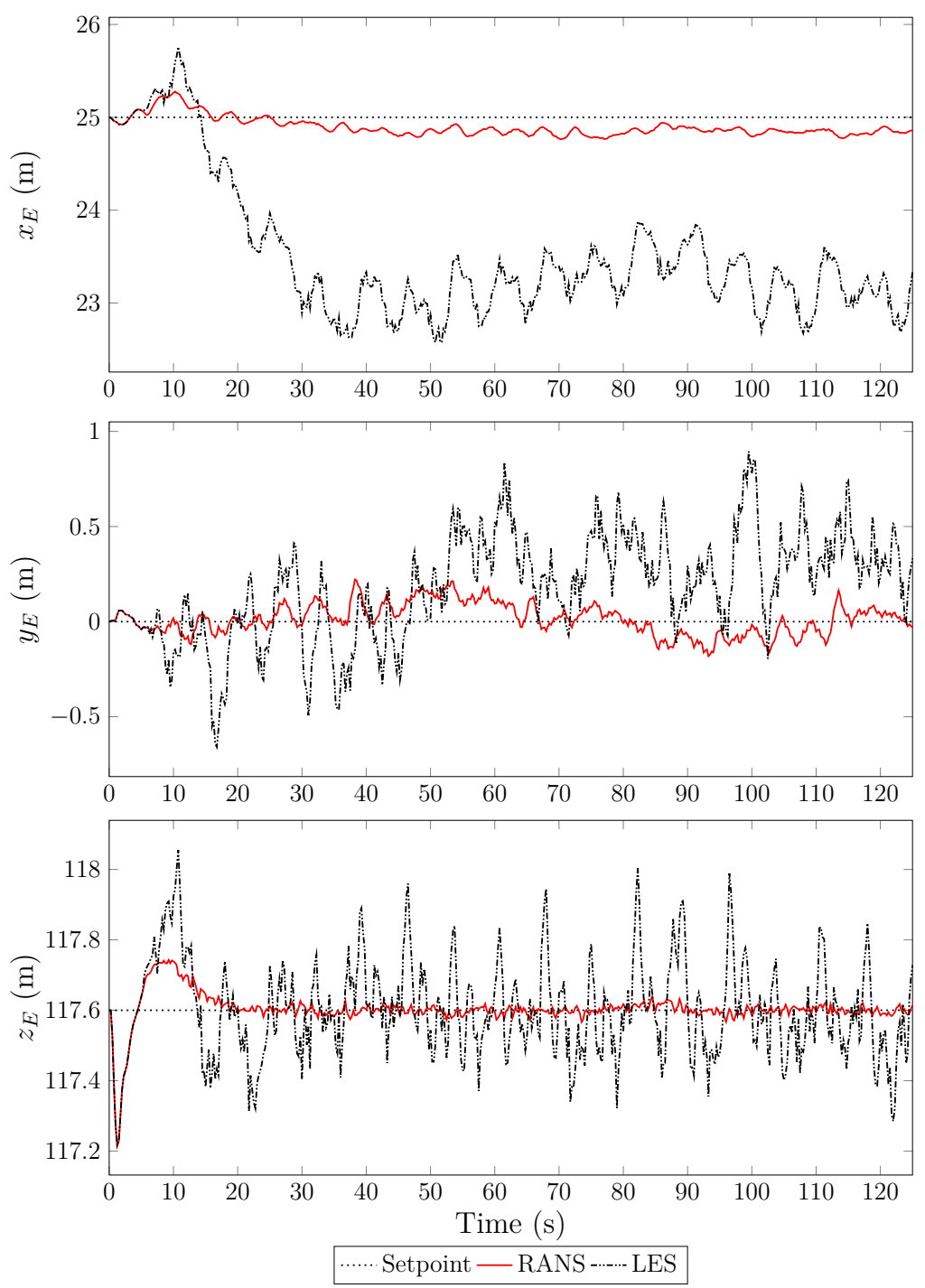

Figure 47: Mission 3 - quadrotor position 
The constant offset wind in the $x_{E}$ and $z_{E}$ directions, coupled with the small oscillations in the $y_{E}$ direction of the RANS database produces variations of only \pm 0.25 body length in the $x_{E}$ and $y_{E}$ directions, and \pm 0.1 body lengths in the $z_{E}$ direction, illustrated in Figure 48. With the higher velocity magnitudes and larger turbulent fluctuations the LES database wind results in position deviations of $+2 /-5$, $+0.5 /-0.5$, and \pm 0.5 body lengths in the $x_{E}, y_{E}$, and $z_{E}$ directions respectively. The large steady state error in the $x_{E}$ direction is clearly visible here and is the main contributor to the LES deviation volume being over 100 times larger than the RANS volume.

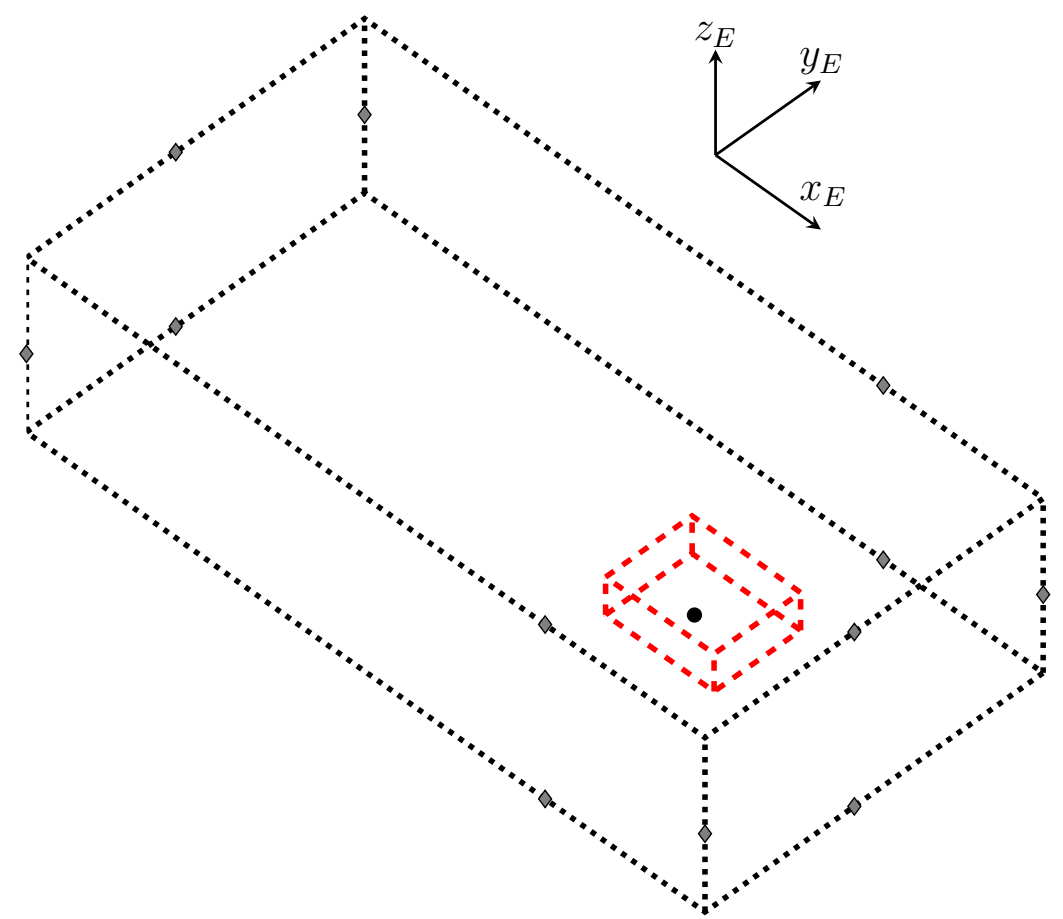

-Setpoint - - - RANS …. LES $\diamond$ Relative Setpoint

Figure 48: Mission 3 - maximum path deviation bounding boxes. The length of the shown coordinate axis represents a quadrotor body length along that axis. 


\subsection{Mission 4 - Ascent Through Wake}

The ascent flight of Mission 4 is designed to test the controller's ability to hold its lateral position while increasing the altitude, simulating a desired situation such as building inspection. The quadrotor is initialized at a height of 5 meters and holds position for 5 seconds without background wind influence. The wind is then quickly ramped up over the next 5 seconds as the quadrotor increases altitude at approximately $1 \mathrm{~m} / \mathrm{s}$ up to 135 meters in the freestream. The results for the fourth mission are shown in Figure 49 with the predicted RANS and LES flight paths shown in scale to the building size and location.

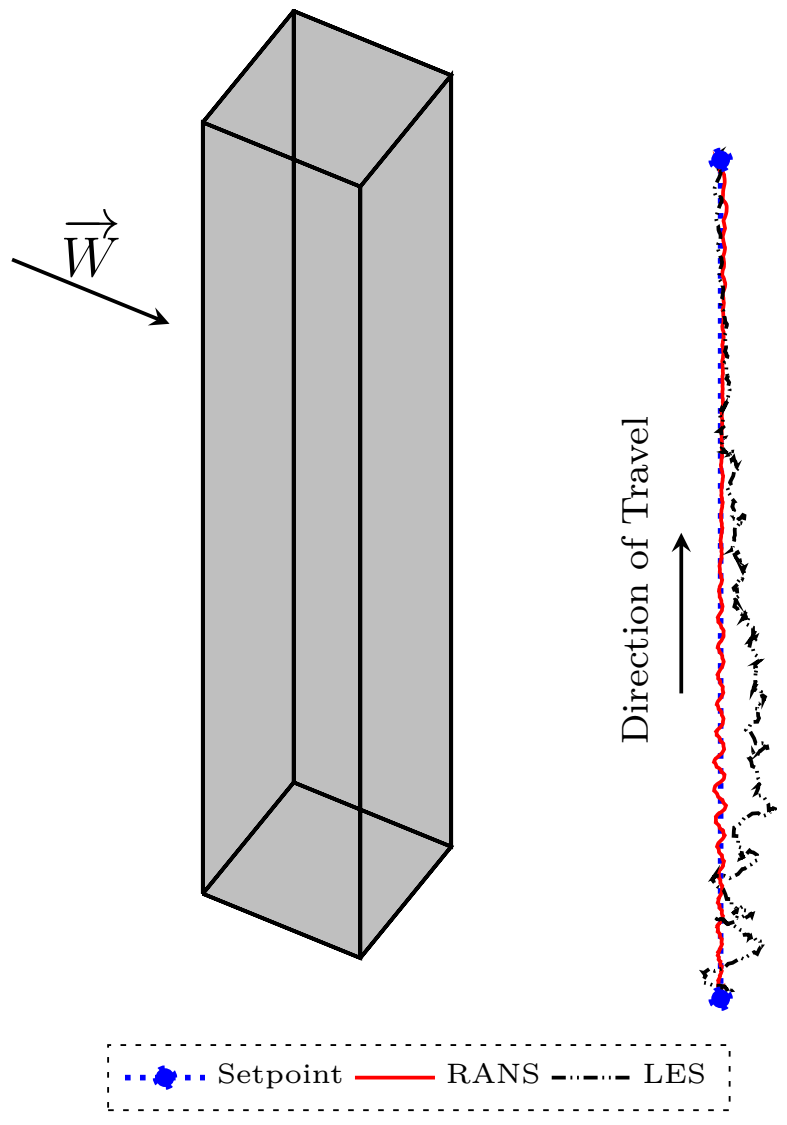

Figure 49: Mission 4 - LES deviations and building 
The wake velocity components the quadrotor experiences over the flight are shown in Figure 50. The winds are now a function of both time and location as the quadrotor's position changes as the mission is flown. In the wake the RANS and LES have the same oscillations observed in the loop interval probe plots (Figures $32 \mathrm{c}$ and $32 \mathrm{~d})$. As the quadrotor ascends these crosswind component oscillations reduce to almost zero ( $W_{y}$ in Figure 50) while the streamwise velocity component increases close to freestream value. The vertical wind component slowly decreases as the quadrotor flies through the roof down wash as shown in Figure 38 until it returns to near zero in the freestream.

The two flight paths are also shown separated into two planes, wind parallel $\left(x_{E^{-}} z_{E}\right)$ and crosswind parallel $\left(y_{E^{-}} z_{E}\right)$ in Figures $51 \mathrm{a}$ and $51 \mathrm{~b}$ to allow for a clearer visualization of the quadrotor's performance. The wind velocities deep in the building wake coupled with the constantly increasing altitude setpoint produce maximum variations of \pm 1 and $+0.25 /-1$ body length in the $x_{E}$ and $y_{E}$ directions respectively when the quadrotor is subjected to the RANS database velocities. Along the same section of flight time the LES wake velocities generate variations of $+12 /-4$ and $+8 /-4.5$ body lengths in the $x_{E}$ and $y_{E}$ directions respectively.

While the velocity is large in the streamwise direction at the end of the mission flight path, the turbulent fluctuations in all three directions reduce to near zero values. Since it is these large scale changes in velocity which have the largest impact on the quadrotor's position, comparable position performance is achieved for both RANS and LES database wind at the end of Mission 4. After reaching the desired 

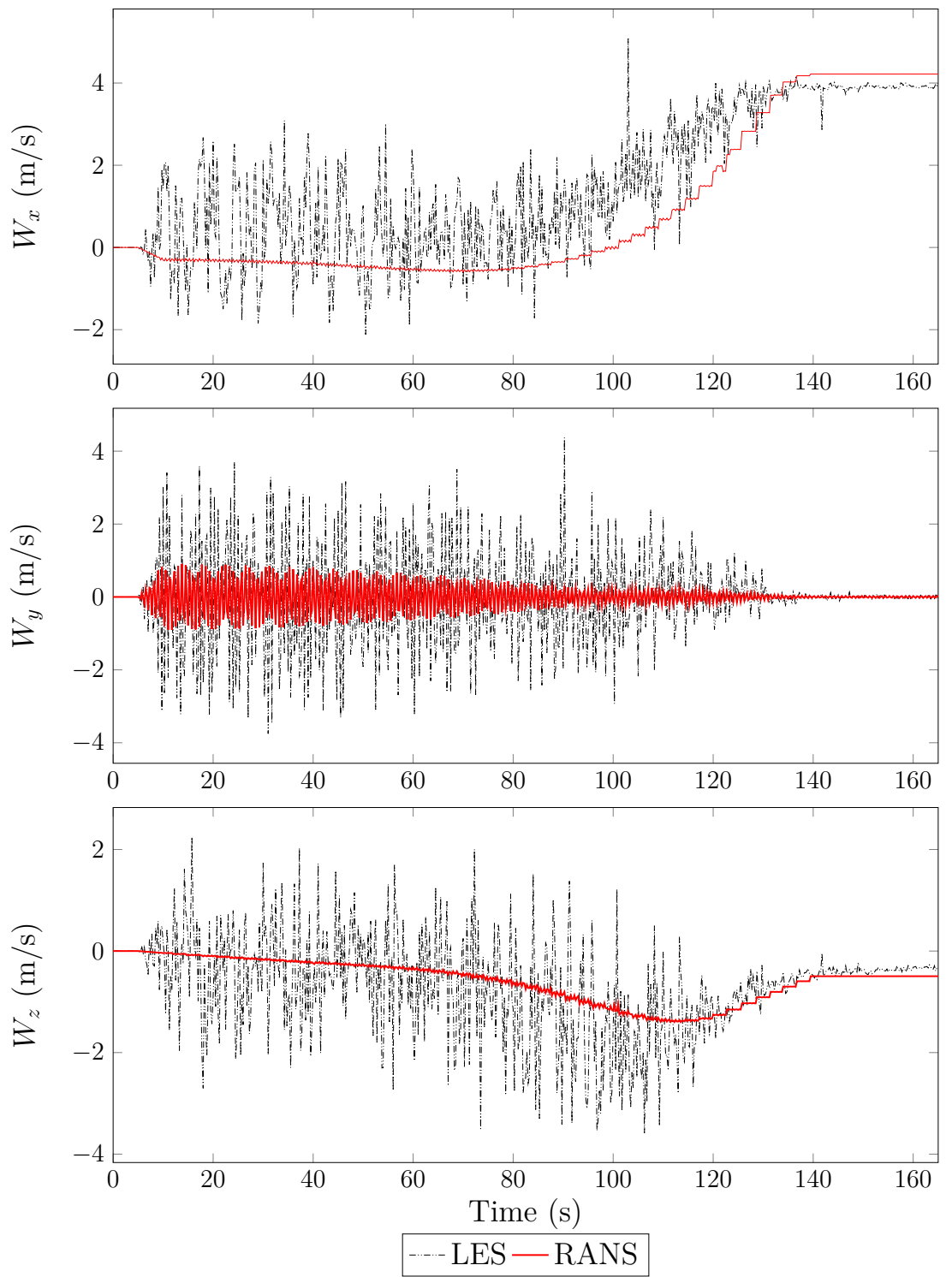

Figure 50: Mission 4 - wind velocity components

$135 \mathrm{~m}$ altitude the quadrotor attempts to maintain it's position for 20 seconds. It is found that the positions for both the RANS and LES background wind conditions converge to produce deviation performance similar to mission 1 of \pm 1 body length in both the $x_{E}$ and $y_{E}$ directions, and \pm 0.25 body lengths in both the $z_{E}$. 


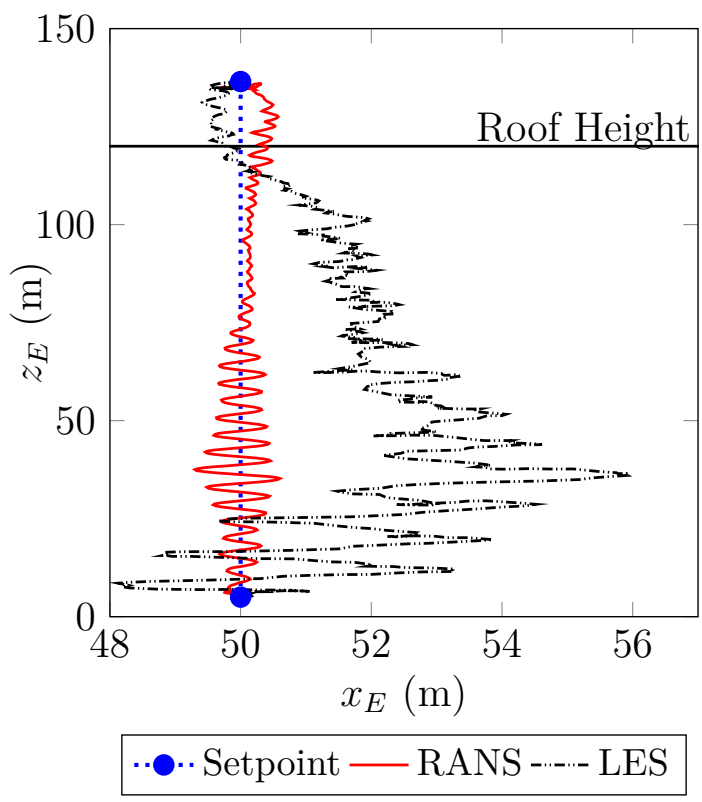

(a) Freestream parallel plane

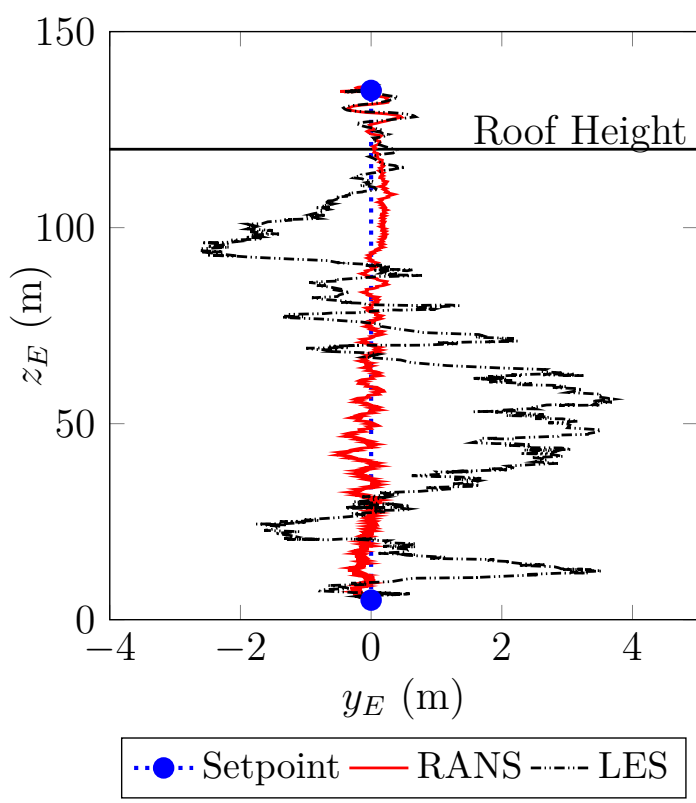

(b) Crosswind parallel plane

Figure 51: Mission 4 - quadrotor position

\subsection{Mission 5 - Crosswind Wake Translation}

The cross wake flight of Mission 5 is designed to test the controller's ability to hold its streamwise position and altitude while traveling in the corsswind direction. This situation is an initial step to move towards the previous work of Galway et al. [13-15] which ultimately consisted of flying through multiple building wakes for realistic flight testing of missions in an urban environment. To study how traveling in and out of the wake impacts the quadrotor's autonomous flight performance, the single building geometry is used as illustrated in Figure 52.

The wake velocity components the quadrotor experiences over the flight are shown in Figure 53. From the streamwise component is is clear when the quadrotor leaves 


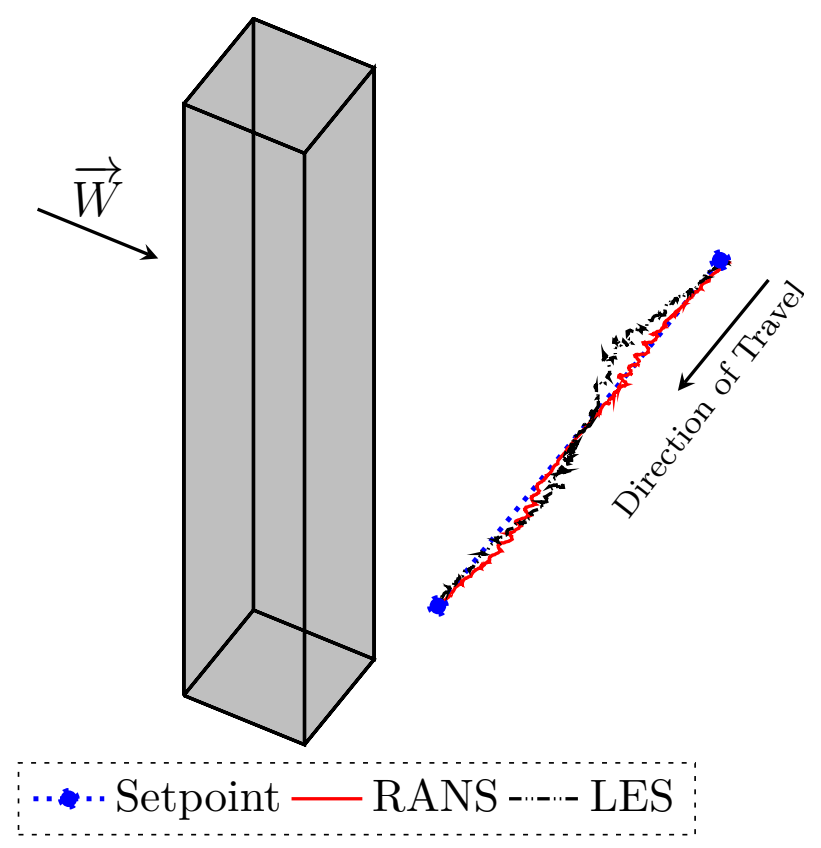

Figure 52: Mission 5 - LES deviations and building

the freestream (with $\mathrm{u}=4 \mathrm{~m} / \mathrm{s})$, at the center of the lower velocity wake $(\mathrm{u} \approx$ $1 \mathrm{~m} / \mathrm{s}$ ), and back to the freesteam. As experienced in Mission 2, the crosswind component exhibits high frequency oscillations and there is a negative ground normal component of $\mathrm{w} \approx 1.5 \mathrm{~m} / \mathrm{s}$ near the center of the building.

The two flight paths are again separated into two planes, wind parallel $\left(x_{E^{-}} y_{E}\right)$ and crosswind parallel $\left(y_{E^{-}} z_{E}\right)$ in Figures 54a and 54b to allow for a clearer visualization of the quadrotor's performance. As the quadrotor enters the wake, the freestream velocity causes the controller to over compensate as it decreases causing a large streamwise deviation upwind of the setpoint. As expected this divergence coupled with the demand of a moving setpoint in the crosswind direction results in deviations in the quadrotor's altitude. The RANS wake field produces 

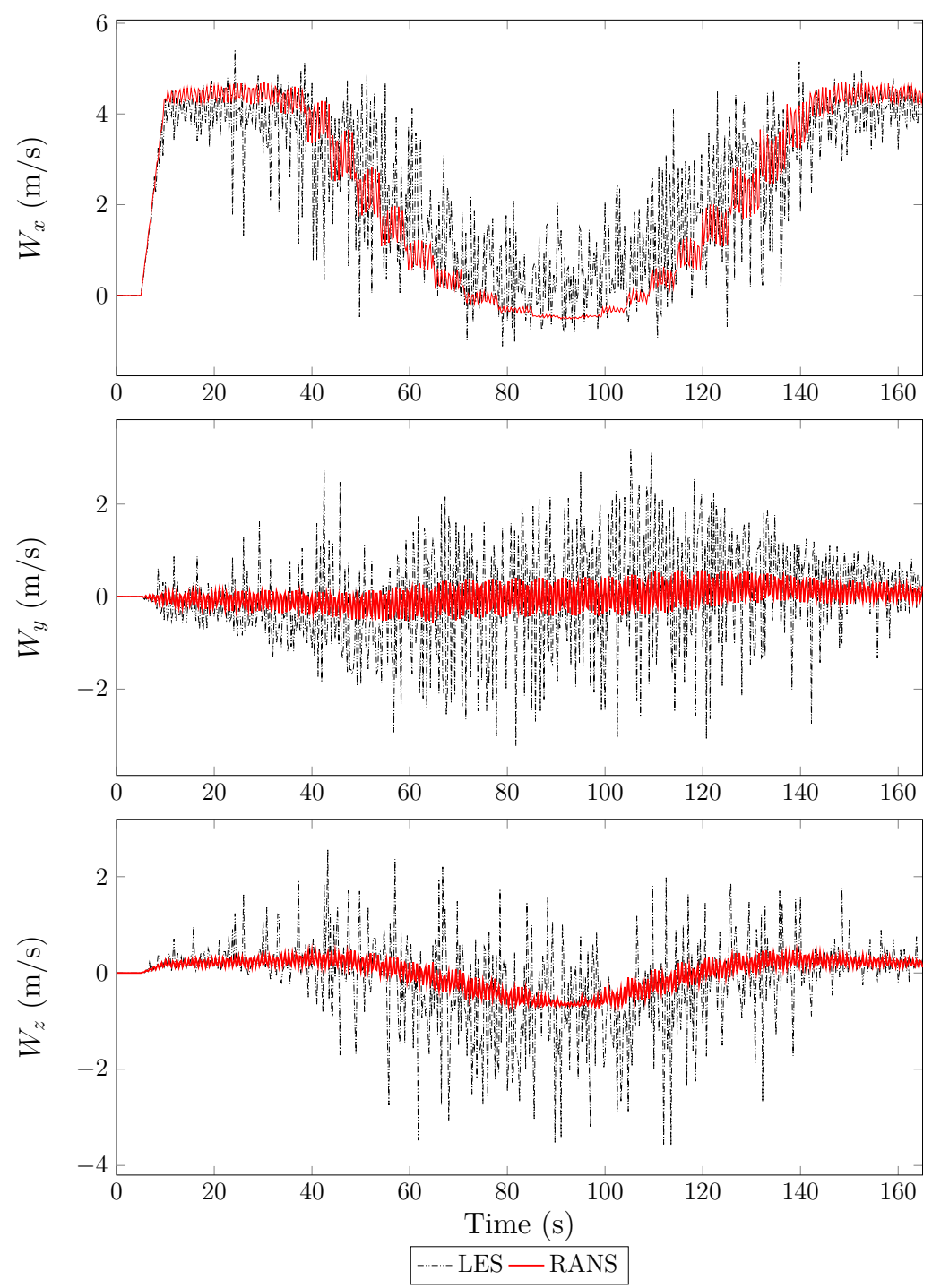

Figure 53: Mission 5 - wind velocity components

deviations of approximately -2 and \pm 0.25 body lengths in the $x_{E}$ and $z_{E}$ directions respectively. When using the LES wake field the quadrotor reaches up to \pm 8 body lengths in the $x_{E}$ as it enters the wake and \pm 2 body lengths in the $z_{E}$ direction.

All three wake velocity components have smaller magnitudes in the wake $($ see $t=$ 
$90 \mathrm{~s}$ in Figure 53), and with a smaller position deviation upon entering the wake the RANS wake field produces a deviation of approximately +0.5 body lengths in the $x_{E}$ direction and on average almost 0 in the $z_{E}$ direction. When using the LES wake field velocities, the quadrotor does not have time to recover from the extreme position deviation before going through the wake. As the controller fights to reach the setpoint it overshoots, producing in $z_{E}$ deviations of \pm 2 body lengths as it begins to experience the increasing streamwise velocity. As the quadrotor leaves the wake this increasing velocity results in an $x_{E}$ deviation of +6 body lengths for the LES wake field. A similar trend is seen with the RANS results but has a lower magnitude with an $x_{E}$ deviation of +4 body lengths downstream of the setpoint.

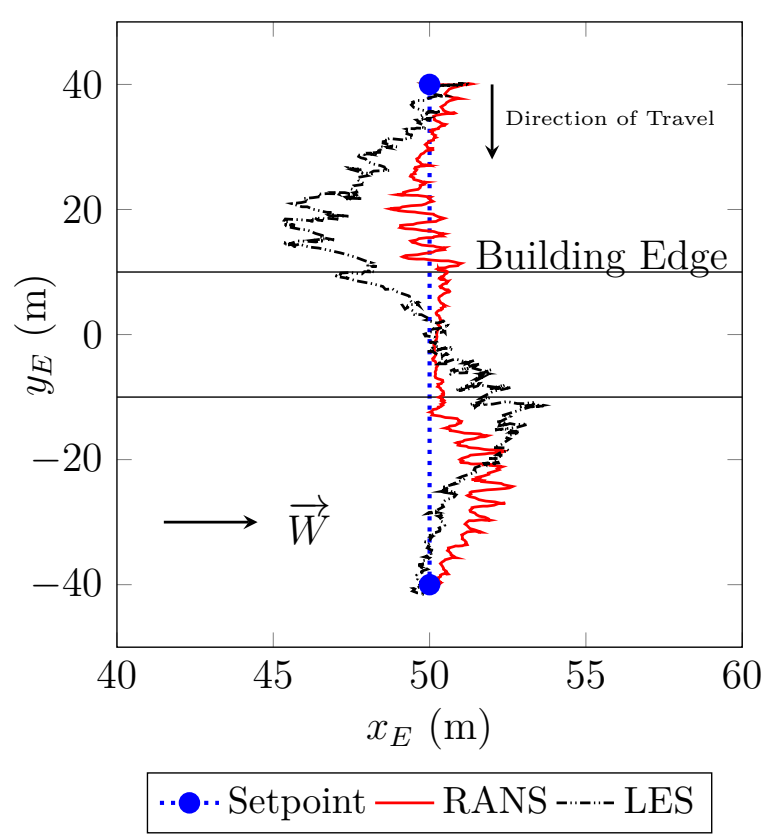

(a) Freestream parallel plane

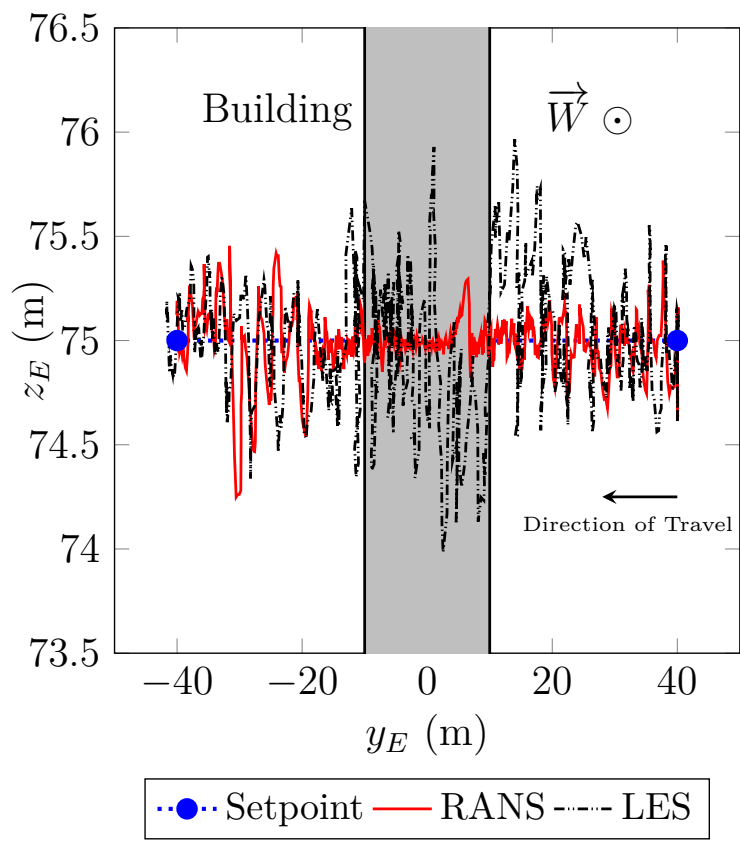

(b) Crosswind parallel plane

Figure 54: Mission 5 - quadrotor position 


\section{Chapter 6}

\section{Conclusions and Recommendations}

\subsection{Conclusions}

Motived by the quadrotor's many uses and increasing popularity, along with growing urban populations, a method is presented for using LES to generate urban wake velocity fields for use in studying wake effects on autonomous quadrotor flight performance. The flow field is generated around a single building in isolation, representing the simplest building block geometry to build up a full urban environment. The wake velocities are stored in a database and accessed by a MATLAB/Simulink flight simulator based on a custom quadrotor platform. This simulator and database are used to study the difference in flight performance between wake fields generated by RANS methods and LES with five flight missions. The mission types and their locations are chosen to study a specific performance ability such as position holding in a turbulent wake or vertical ascent in the wake. 
The control case of position holding with a constant prescribed value, RANS, and LES freestream background wind conditions produce similar maximum deviations as expected. While the RANS and LES deviations volumes are slightly larger, due to the low level freestream turbulence and $0.4 \mathrm{~m} / \mathrm{s} W_{z}$ wind component, both CFD methods produce similar results and can hold position in all three directions within approximately \pm 1.5 body lengths.

When the quadrotor has any interaction with the building wake the maximum deviation volumes between RANS and LES background wind can differ by 2 orders of magnitude. This is clearly seen when holding position at both mid and roof height downstream of the building with bounding deviation volumes of $0.03 \mathrm{~m}^{3}$ and $0.04 \mathrm{~m}^{3}$ with a RANS background wind while the LES will result in volumes of $7.2 \mathrm{~m}^{3}$ and $4.3 \mathrm{~m}^{3}$. This substantial increase is a result of the resolved turbulent fluctuations and higher velocity magnitudes in the LES wake field otherwise averaged out when using the RANS wake modeling technique. In addition to the larger bounding volumes these random non-isotropic fluctuations cause skewed deviations by as much as 5 to 1 in different axis as the controller attempts to maintain position at the setpoint. Similar performance is observed when the quadrotor is translating vertically or horizontally through the building wake. Using a RANS background wake field produces deviation volumes of $172 \mathrm{~m}^{3}$ and $386 \mathrm{~m}^{3}$ while the same flight under LES wind conditions yields $13,566 \mathrm{~m}^{3}$ and 1,436 $\mathrm{m}^{3}$.

Since it takes approximately 12 days to produce a 30 second LES wake field and only 16 hours using RANS, it is tempting to use RANS to model the urban wake 
fields, considering the number of different building block simulations required to generate a complete urban environment. However, the transient turbulent wake field modeled by LES more accurately reflects the wake fields present behind real world structures. Because of this, and coupled with resulting quadrotor flight performance between RANS and LES, it is recommend to only use LES generated wake fields in the pursuit of designing and testing autonomous control algorithms for multirotor UAVs on the order of $0.5 \mathrm{~m}$ in size and $2 \mathrm{~kg}$ in mass.

\subsection{Recommendations}

The first step in future development is to continue the replication of Galway et al. [13-15] methods for application to the SUA platform, like a quadrotor, by expanding the urban wake database with buildings at different freestream orientations and more complex geometries such as urban canyons. This can then be expanded further by applying the selection algorithm of Galway to build up an urban environment using the CFD building blocks to simulate flight with multiple buildings. In parallel, the physical quadrotor can be finalized and a mission planned with the goal of collecting experimental data of the quadrotor's autonomous performance with a single building geometry.

The capabilities of the flight simulator can be expanded with a multi-point model to study the flight performance with aerodynamic moments induced by the wind effects acting on other parts of the quadrotor, such as the arms/propellers. Currently the wind force components are applied to the center of gravity. However a multi-point 
model would require a finer mesh density or 3D interpolation between the existing database points. Initial testing indicates interpolating between the current mesh density does not produce a discernible difference in either wake the velocity components or the quadrotor's position when the forces are applied only to the center of gravity. While interpolating cannot produce eddy motion, as this is limited implicitly by the mesh spacing, it may provide useful information for implementing and testing a multi-point quadrotor model. Finally the flight simulator can be further developed to include multiple quadrotors for the design and testing of vehicle swarming in urban environments. 


\section{List of References}

[1] International Civil Aviation Organization. Unmanned aircraft systems (UAS). International Civil Aviation Organization, Montréal. ISBN 9789292317515 9292317512 (2011).

[2] "Urban Development." Http://data.worldbank.org/topic/urban-development (2014).

[3] D. W. Murphy and J. Cycon. "Applications for mini VTOL UAV for law enforcement." In "Enabling Technologies for Law Enforcement and Security," pages 35-43. International Society for Optics and Photonics (1999).

[4] T. Hegazy, B. Ludington, and G. Vachtsevanos. "Reconnaissance and surveillance in urban terrain with unmanned aerial vehicles." In "Proceedings of 16 th IFAC World Congress," pages 4-8 (2005).

[5] R. L. Mota, L. F. Felizardo, E. H. Shiguemori, A. C. Ramos, and F. Mora-Camino. "Expanding Small UAV Capabilities with ANN: A Case Study for Urban Areas Inspection." British Journal of Applied Science $\mathcal{E}_{3}$ Technology 4(2), 387-398 (2014).

[6] AeroVironment. "Qube Public Safety UAS - AeroVironment, Inc." Http://www.avinc.com/public-safety/qube (2015).

[7] T. Coyle and . a. p. S. M. V. Blog. "Comparing traditional CSI procedures with UAV Mapping." Http://diydrones.com/profiles/blogs/comparingtraditional-csi-procedures-with-uav-mapping (2014). 
[8] “Amazon Prime Air." Http://www.amazon.com/b?node=8037720011 (2014).

[9] T. A. Press. "DHL begins drone delivery in Germany." Http://www.cbc.ca/1.2777647 (2014).

[10] L. Kelion. "Alibaba begins drone delivery trials." Http://www.bbc.com/news/technology-31129804 (2015).

[11] F. P. Staff. "Pizza-delivering drones? Domino's shows off unmanned aircraft that delivers customer orders." Financial Post Http://business.financialpost.com/2013/06/06/dominos-drone-pizza-delivery/ (2013).

[12] J. Etele. "Overview of Wind Gust Modelling with Application to Autonomous Low-Level UAV Control." DRDC Contract Report, Carleton University (2006).

[13] D. Galway, J. Etele, and G. Fusina. Urban Wind Modeling with Application to Autonomous Flight. Masters Thesis, Carleton University (2009).

[14] D. Galway, J. Etele, and G. Fusina. "Modeling of Urban Wind Field Effects on Unmanned Rotorcraft Flight." Journal of Aircraft 48(5), 1613-1620. ISSN 0021-8669, 1533-3868 (2011).

[15] D. Galway, J. Etele, and G. Fusina. "Development and implementation of an urban wind field database for aircraft flight simulation." Journal of Wind Engineering and Industrial Aerodynamics 103, 73-85. ISSN 0167-6105 (2012).

[16] M. Orr, S. Rasmussen, E. Karni, and W. Blake. "Framework for developing and evaluating MAV control algorithms in a realistic urban setting." In "American Control Conference, 2005. Proceedings of the 2005," pages 4096-4101 vol. 6 (2005).

[17] M. Kothari, I. Postlethwaite, and D.-W. Gu. "UAV Path Following in Windy Urban Environments." Journal of Intelligent \& Robotic Systems ISSN 0921-0296, 1573-0409 (2013).

[18] E. Semsch, M. Jakob, D. Pavlicek, and M. Pechoucek. "Autonomous UAV Surveillance in Complex Urban Environments." In "IEEE/WIC/ACM 
International Joint Conferences on Web Intelligence and Intelligent Agent Technologies, 2009. WI-IAT '09," volume 2, pages 82-85 (2009).

[19] S. Waslander and C. Wang. "Wind Disturbance Estimation and Rejection for Quadrotor Position Control." American Institute of Aeronautics and Astronautics. ISBN 978-1-60086-979-2 (2009).

[20] S. A. Raza and J. Etele. "Simulation tool for testing and validating uav autopilots in wind gust environments." AIAA Atmospheric Flight Mechanics Conference and Exhibit (2012).

[21] Pratt. "MQ-1 Predator unmanned aircraft." Http://commons.wikimedia.org/wiki/File:MQ-1_Predator_unmanned_aircraft.jpg (2008).

[22] C. Slattery. "Northrop Grumman MQ-4c Triton." Http://en.wikipedia.org/w/index.php?title=Northrop_Grumman_MQ-4C_Triton\&oldid $=642760198$ (2015).

[23] Jrfreeland. "Northrop Grumman MQ-8 Fire Scout." Http://en.wikipedia.org/w/index.php?title=Northrop_Grumman_MQ-8_Fire_Scout\&oldid=640305274 (2015).

[24] MilborneOne. "Schiebel Camcopter S-100." Http://en.wikipedia.org/w/index.php?title=Schiebel_Camcopter_S-100\&oldid=643663322 (2015).

[25] KrisfromGermany. "EMT Aladin." Http://en.wikipedia.org/w/index.php?title=EMT_Aladin\&oldid=542404626 (2015).

[26] Dkroetsch. "Miniature UAV." Http://en.wikipedia.org/w/index.php?title $=$ Miniature_UAV\&oldid $=644858733$ (2015). 
[27] TFCforever. "de Bothezat helicopter." Http://en.wikipedia.org/w/index.php?title=De_Bothezat_helicopter\&oldid=609142829 (2015).

[28] Halftermeyer. "AR.Drone." Http://pl.wikipedia.org/w/index.php?title=AR.Drone\&oldid=41440599 (2015).

[29] F. M. Hoblit. Gust Loads on Aircraft: Concepts and Applications. AIAA Education Series. American Institute of Aeronautics and Astronautics, Washington, D.C. ISBN 0-930403-45-2 (1988).

[30] J. R. Richardson, E. M. Atkins, P. T. Kabamba, and A. R. Girard. "Envelopes for Flight Through Stochastic Gusts." Journal of Guidance, Control, and Dynamics 36(5), 1464-1476. ISSN 0731-5090, 1533-3884 (2013).

[31] J. W. Langelaan. "Long distance/duration trajectory optimization for small uavs." In "AIAA Guidance, Navigation and Control Conference and Exhibit, Hilton Head, SC," (2007).

[32] R. E. Britter and S. R. Hanna. "Flow and Dispersion in Urban Areas." Annual Review of Fluid Mechanics 35(1), 469-496 (2003).

[33] R. B. Stull. An Introduction to Boundary Layer Meteorology. Springer, New York, softcover reprint of the original 1st ed. 1988 edition edition. ISBN 9789027727695 (1988).

[34] M. Bottema. "Urban roughness modelling in relation to pollutant dispersion." Atmospheric Environment 31(18), 3059-3075. ISSN 1352-2310 (1997).

[35] X. Tapia. Modeling wind flow over complex terrain using OpenFoam. Masters Thesis, University of Gavle (2009).

[36] J. Wieringa, A. Davenport, S. S. Grimmond, and T. Oke. "New revision of Davenport Roughness Classification." Proc., 3EACWE, Eindhoven, The Netherlands (2001). 
[37] J. Escareno, S. Salazar, H. Romero, and R. Lozano. "Trajectory Control of a Quadrotor Subject to 2d Wind Disturbances." Journal of Intelligent \& Robotic Systems 70(1-4), 51-63. ISSN 0921-0296, 1573-0409 (2012).

[38] J. Guerrero, J. Escareno, and Y. Bestaoui. "Quad-rotor MAV trajectory planning in wind fields." In "2013 IEEE International Conference on Robotics and Automation (ICRA)," pages 778-783 (2013).

[39] Y. Chen, Y. He, and M. Zhou. "Modeling and Control of a Quadrotor Helicopter System under Impact of Wind Field." (2013).

[40] B. Ferrier, A. Sehgal, and R. Ernst. "Fire Scout UAV Launch and Recovery System Performance Improvement." (2014).

[41] P. Gousseau, B. Blocken, and G. van Heijst. "CFD simulation of pollutant dispersion around isolated buildings: On the role of convective and turbulent mass fluxes in the prediction accuracy." Journal of Hazardous Materials 194, 422-434. ISSN 03043894 (2011).

[42] A. Walton and A. Y. S. Cheng. "Large-eddy simulation of pollution dispersion in an urban street canyonPart II: idealised canyon simulation." Atmospheric Environment 36(22), 3615-3627. ISSN 1352-2310 (2002).

[43] S. M. Salim, R. Buccolieri, A. Chan, and S. Di Sabatino. "Numerical simulation of atmospheric pollutant dispersion in an urban street canyon: Comparison between RANS and LES." Journal of Wind Engineering and Industrial Aerodynamics 99(2-3), 103-113. ISSN 01676105 (2011).

[44] T. Tamura. "Large eddy simulation on building aerodynamics." In "Proceedings of the seventh Asia-Pacific Conference on Wind Engineering, pp131-157," (2009).

[45] J. He and C. C. S. Song. "Evaluation of pedestrian winds in urban area by numerical approach." Journal of Wind Engineering and Industrial Aerodynamics 81(1-3), 295-309. ISSN 0167-6105 (1999).

[46] M. C. Bogstad, W. G. Habashi, I. Akel, D. Ait-Ali-Yahia, N. Giannias, and V. Longo. "Computational-Fluid-Dynamics Based Advanced Ship-Airwake 
Database for Helicopter Flight Simulators." Journal of Aircraft 39(5), 830-838. ISSN 0021-8669, 1533-3868 (2002).

[47] S. Zan. "Technical Comment on "Computational-Fluid-Dynamics Based Advanced Ship-Airwake Database for Helicopter Flight Simulation"." Journal of Aircraft 40(5), 1007-1007. ISSN 0021-8669, 1533-3868 (2003).

[48] J. S. Forrest, I. Owen, G. D. Padfield, and S. J. Hodge. "Ship-Helicopter Operating Limits Prediction Using Piloted Flight Simulation and Time-Accurate Airwakes." Journal of Aircraft 49(4), 1020-1031. ISSN 0021-8669, 1533-3868 (2012).

[49] C. Crozon, R. Steijl, and G. N. Barakos. "Numerical Study of Helicopter Rotors in a Ship Airwake." Journal of Aircraft 51(6), 1813-1832. ISSN 0021-8669, 1533-3868 (2014).

[50] J. V. Muijden, O. Boelens, J. v. d. Vorst, and J. Gooden. "Computational ship airwake determination to support helicopter-ship dynamic interface assessment." In "21st AIAA Computational Fluid Dynamics Conference," American Institute of Aeronautics and Astronautics (2013).

[51] R. Martinuzzi and C. Tropea. "The flow around surface-mounted, prismatic obstacles placed in a fully developed channel flow (Data Bank Contribution)." Journal of Fluids Engineering 115(1), 85-92 (1993).

[52] S. Dutta, P. K. Panigrahi, and K. Muralidhar. "Experimental investigation of flow past a square cylinder at an angle of incidence." Journal of engineering mechanics 134(9), 788-803 (2008).

[53] K. Elshorbagy, E. Wahba, and R. Afify. "Visualization versus CFD Simulation of Laminar Flow past Bluff Body." In "ICFD10," Egypt (2010).

[54] S. Houda, N. Zemmouri, A. Hasseine, R. Athmani, and R. Belarbi. "A CFD Model for Simulating Ubran Flow in Complex Morphological Street Network." The Online Journal of Science and Technology 2(1), 1-10 (2012).

[55] J. Labovský and u. Jelemenský. "CFD-based atmospheric dispersion modeling in real urban environments." Chemical Papers 67(12), 1495-1503. ISSN 0366-6352, 1336-9075 (2013). 
[56] OpenFOAM Foundation. "OpenFOAM User Guide.” (2014).

[57] H. Versteeg and W. Malalasekera. An Introduction to Computational Fluid Dynamics: The Finite Volume Method. Prentice Hall, Harlow, England ; New York, 2 edition edition. ISBN 9780131274983 (2007).

[58] E. de Villers. The Potential of Large Eddy Simulation for the Modeling of Wall Bounded Flows. Ph.D. thesis, Imperial College of Science, Technology and Medicine (2006).

[59] O. Reynolds. "An Experimental Investigation of the Circumstances Which Determine Whether the Motion of Water Shall Be Direct or Sinuous, and of the Law of Resistance in Parallel Channels." Philosophical Transactions of the Royal Society of London 174, 935-982. ISSN 0261-0523 (1883).

[60] J. M. Mcdonough. Introductory Lectures on Turbulence Physics, Mathematics and Modeling (2004).

[61] H. Tennekes and J. L. Lumley. A First Course in Turbulence. MIT Press. ISBN 9780262200196 (1972).

[62] B. E. Launder and D. B. Spalding. "The numerical computation of turbulent flows." Computer Methods in Applied Mechanics and Engineering 3(2), 269-289. ISSN 0045-7825 (1974).

[63] S. B. Pope. Turbulent Flows. Cambridge University Press. ISBN 9780521598866 (2000).

[64] A. Leonard. Energy Cascade in Large-Eddy Simulations of Turbulent Fluid Flows, volume 1 of Turbulent Diffusion in Environmental Pollution (1974).

[65] H. Lu, C. J. Rutland, and L. M. Smith. "A priori tests of one-equation LES modeling of rotating turbulence." Journal of Turbulence page N37 (2007).

[66] Charlesreid1. "Filter (large eddy simulation)." Http://en.wikipedia.org/wiki/Filter_\%28large_eddy_simulation\%29 (2010).

[67] P. Sagaut. Large Eddy Simulation for Incompressible Flows - An Introduction. Scientific Computation. Springer, 3rd edition. ISBN 978-3-540-26344-9 (2006). 
[68] A. Yoshizawa and K. Horiuti. "A statistically-derived subgrid-scale kinetic energy model for the large-eddy simulation of turbulent flows." Journal of the Physical Society of Japan 54(8), 2834-2839 (1985).

[69] S. Krajnovic and L. Davidson. "Large-Eddy Simulation of the Flow Around a Bluff Body." AIAA Journal 40(5), 927-936. ISSN 0001-1452 (2002).

[70] J. Franke, A. Hellsten, H. Schlunzen, and B. Carissimo, editors. Proceedings / International Workshop on Quality Assurance of Microscale Meteorological Models Cost action 732 in combination with the European Science Foundation at Hamburg, Germany, July 28/29, 2005. Univ., Meteorological Inst., Centre for Marine and Atmospheric Sciences, Hamburg. ISBN 3000183124 9783000183126 (2005).

[71] Y. Tominaga, A. Mochida, R. Yoshie, H. Kataoka, T. Nozu, M. Yoshikawa, and T. Shirasawa. "AIJ guidelines for practical applications of CFD to pedestrian wind environment around buildings." Journal of Wind Engineering and Industrial Aerodynamics 96(10-11), 1749-1761. ISSN 01676105 (2008).

[72] I. B. Celik, Z. N. Cehreli, and I. Yavuz. "Index of Resolution Quality for Large Eddy Simulations." Journal of Fluids Engineering 127(5), 949-958. ISSN 0098-2202 (2005).

[73] P. Gousseau, B. Blocken, and G. van Heijst. "Quality assessment of Large-Eddy Simulation of wind flow around a high-rise building: Validation and solution verification." Computers \&f Fluids 79, 120-133. ISSN 00457930 (2013).

[74] T. Meng and K. Hibi. "Turbulent measurements of the flow field around a high-rise building." Journal of Wind Engineering Japan (76), 55-64. (in Japanese) (1998).

[75] J. Jeong and F. Hussain. "On the identification of a vortex." Journal of Fluid Mechanics 285, 69-94. ISSN 1469-7645 (1995).

[76] J. C. R. Hunt, A. A. Wray, and P. Moin. "Eddies, streams, and convergence zones in turbulent flows." In "Summer Program 1988," Center for Turbulence Research (1988). 
[77] V. Kolar. "Vortex identification: New requirements and limitations." International Journal of Heat and Fluid Flow 28(4), 638-652. ISSN 0142727X (2007).

[78] G. R. Meneely and N. L. Kaltreider. "The volume of the lung determined by helium dilution. Description of the method and comparison with other procedures." Journal of Clinical Investigation 28(1), 129 (1949).

[79] C. K. Choi and D. K. Kwon. "Determination of the Strouhal number based on the aerodynamic behavior of rectangular cylinders." Wind and Structures 3(3), 209-220 (2000).

[80] K. Shimada and T. Ishihara. "Application of a modified k-e model to the prediction of aerodynamic characteristics of rectangular cross-section cylinders." Journal of Fluids and Structures 16(4), 465 - 485. ISSN 0889-9746 (2002).

[81] O. Almeida, S. Mansur, and A. Silveira-Neto. "On the flow past rectangular cylinders: physical aspects and numerical simulation." Therm. Eng 7(1), 55-64 (2008).

[82] M. Sarioglu and T. Yavuz. "Vortex shedding from circular and rectangular cylinders placed horizontally in a turbulent flow." Turkish Journal of Engineering and Environmental Sciences 24(4), 217-228 (2000).

[83] B. Etkin and L. D. Reid. Dynamics of Flight: Stability and Control. Wiley, New York, 3 edition edition. ISBN 9780471034186 (1995). 


\section{Appendix A}

\section{LES Standard Working Directory}

This appendix details the setup and required file structure to perform a LES with OpenFOAM as outlined in Chapter 2. A standard working directory (SWD) consisting of files and scripts is used to simplify the user work flow for running future simulations to expand the urban wind database. Figure 55 illustrates the simplicity and few steps required by the user to run the LES and Figure 56 shows the full required SWD.

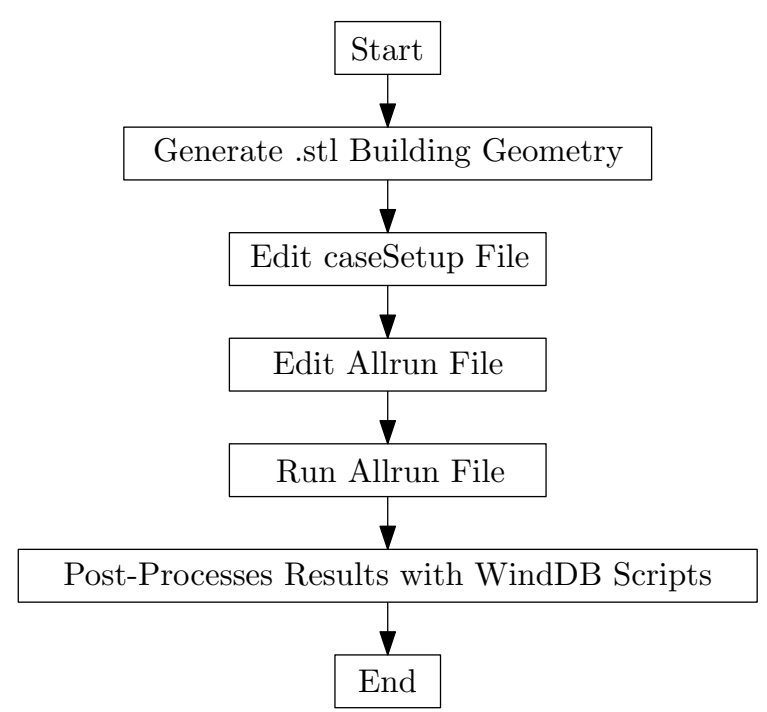

Figure 55: OpenFOAM LES user workflow 
SWD/

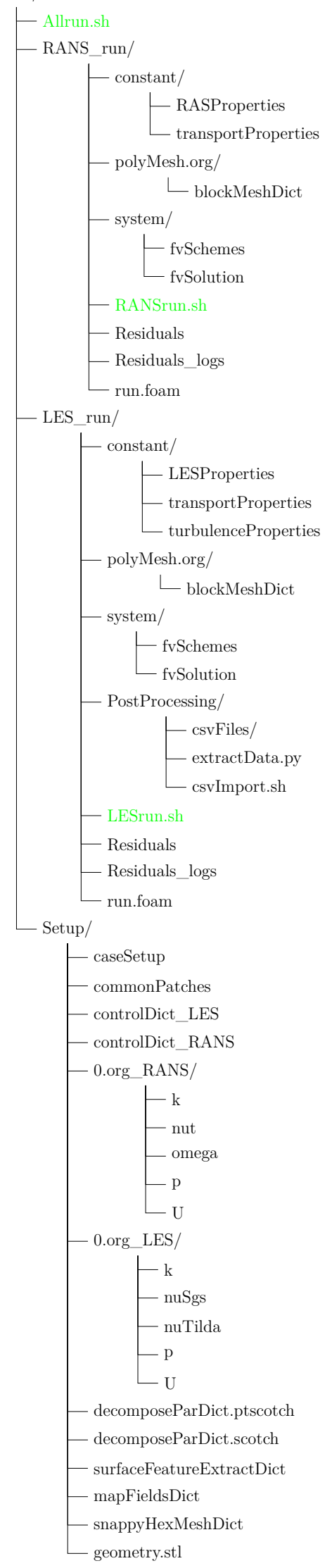

Figure 56: Outline of the standard OpenFOAM working directory 
A zip and tar containing the latest version of the SWD can be downloaded with the following URL: https://github.com/sutherlandm/tara_les-swd/releases. The Allrun script is used to control which parts of the RANS and LES cases are performed, shown in Listing A.1.

Listing A.1: Allrun.sh

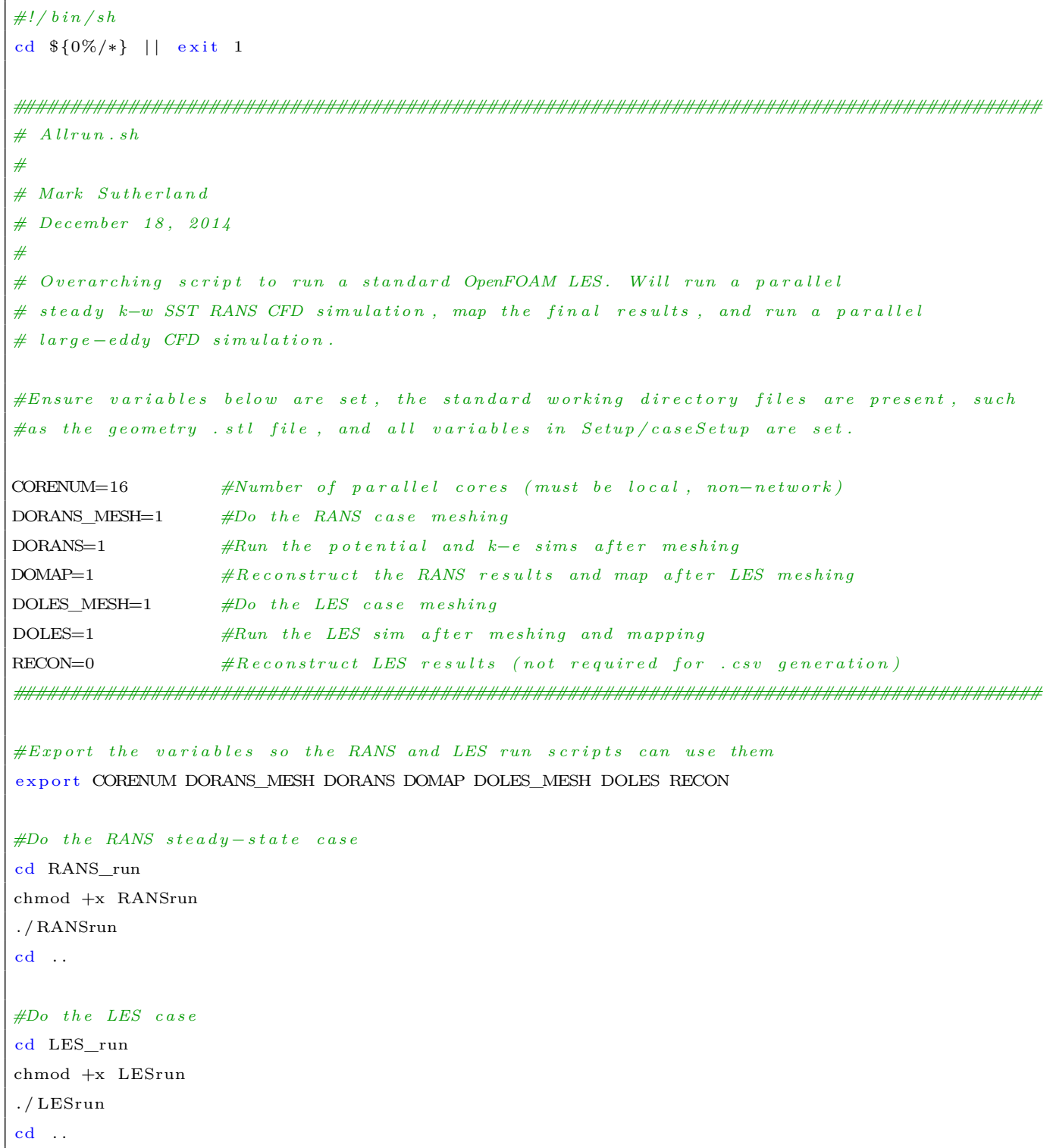


From the Allrun script and the SWD it is seen a RANSrun script is first called to control the RANS simulation based on the flags set in the Allrun, such as meshing and mapping. The RANSrun script is shown below in Listing A.2 with detailed comments explaining the clean up, setup, meshing, running, and reconstruction of the steady RANS case.

Listing A.2: RANSrun.sh

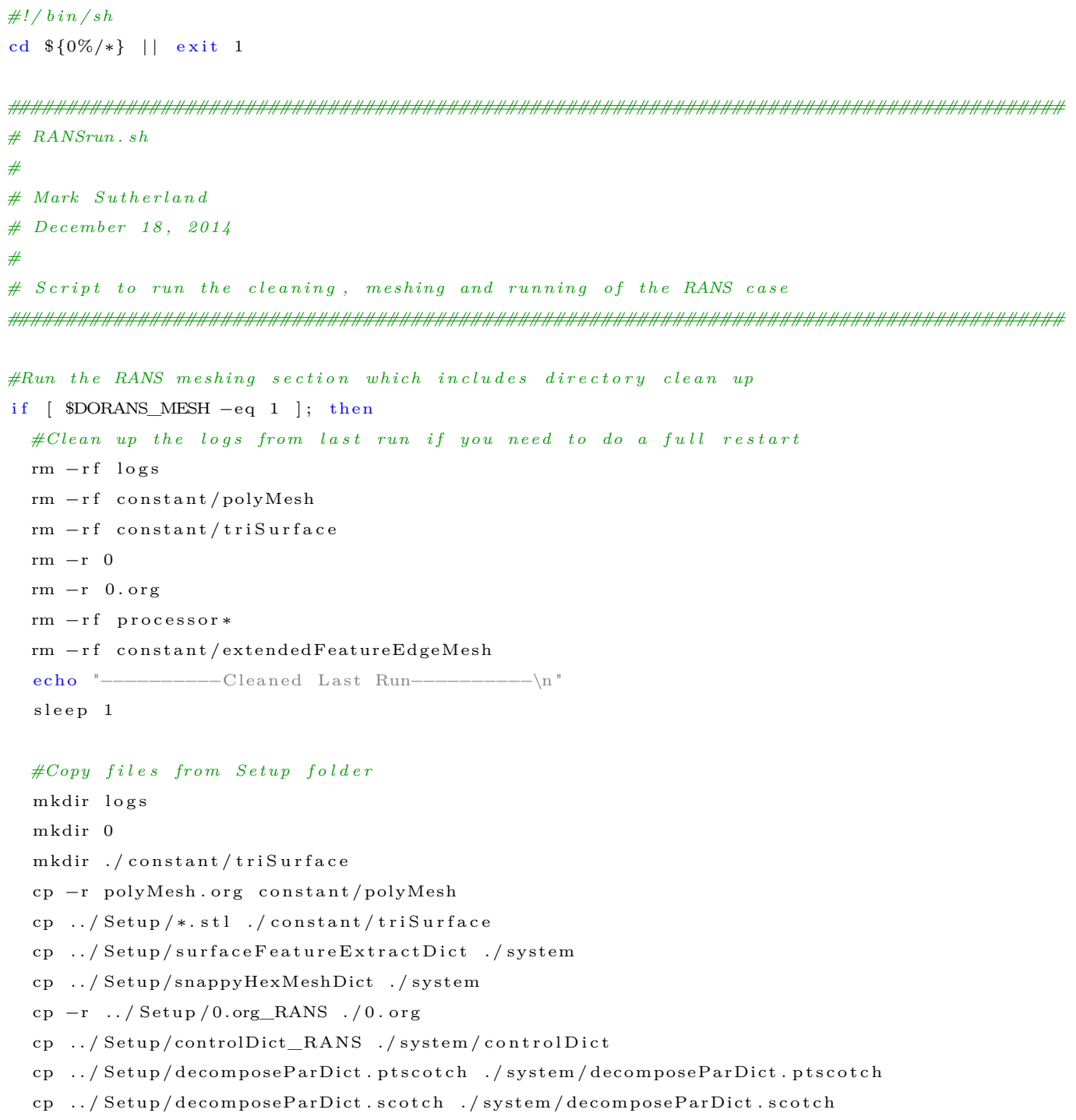




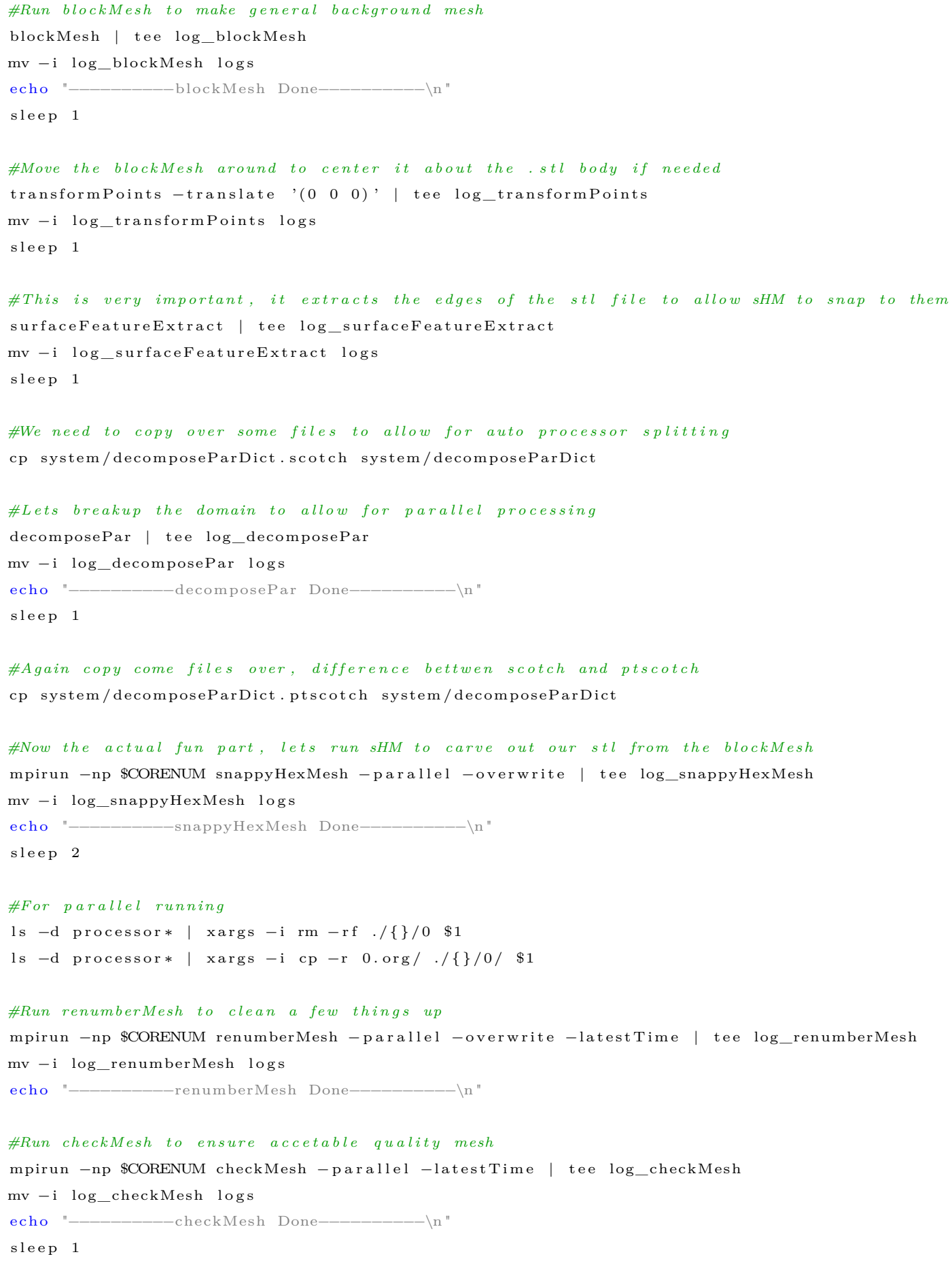




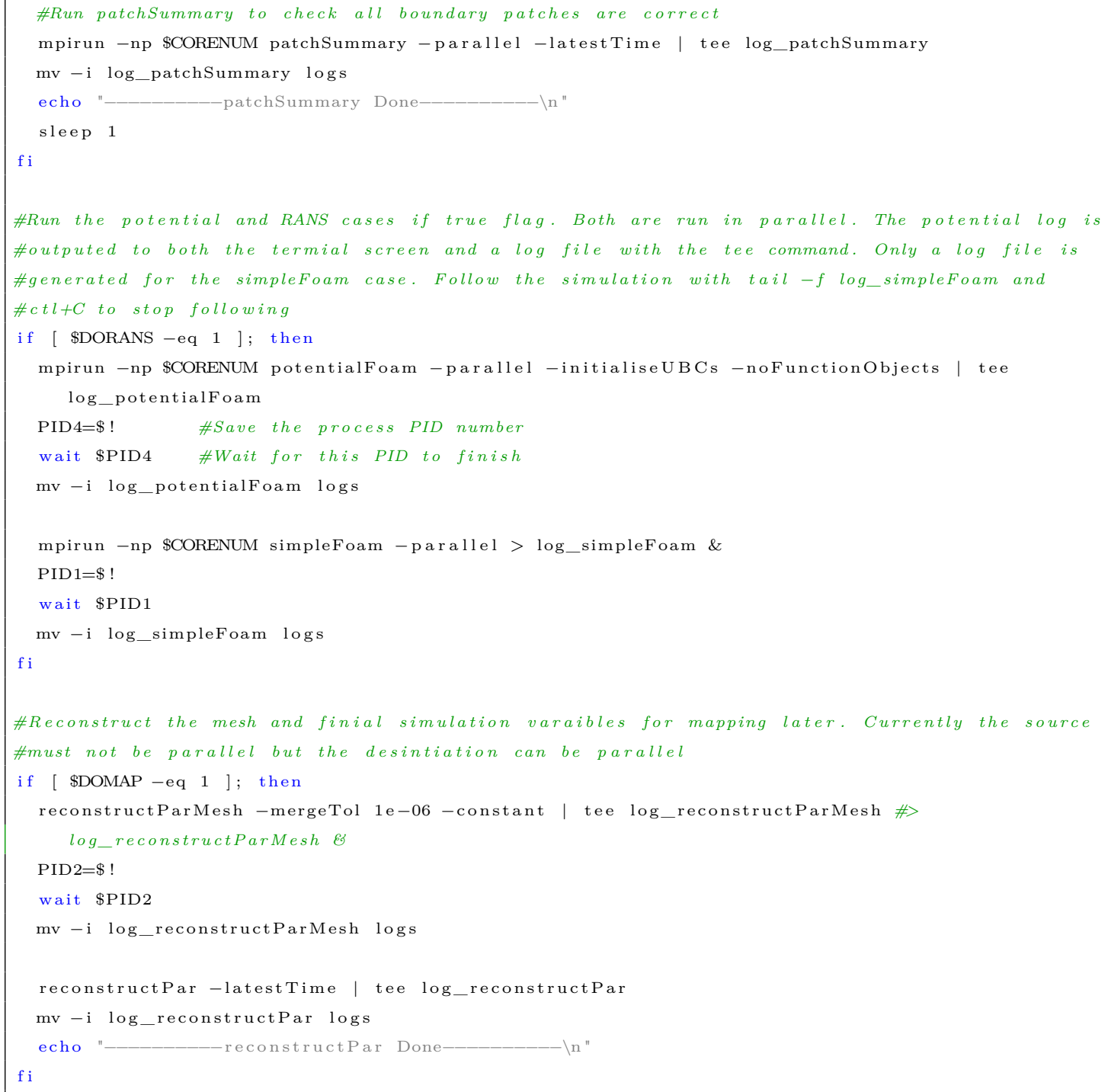

A similar script is then called for the LES case, as detailed in Listings A.3. Again the file is commented to outline the various steps used to ultimately generate the urban wake velocity fields. 
Listing A.3: LESrun.sh

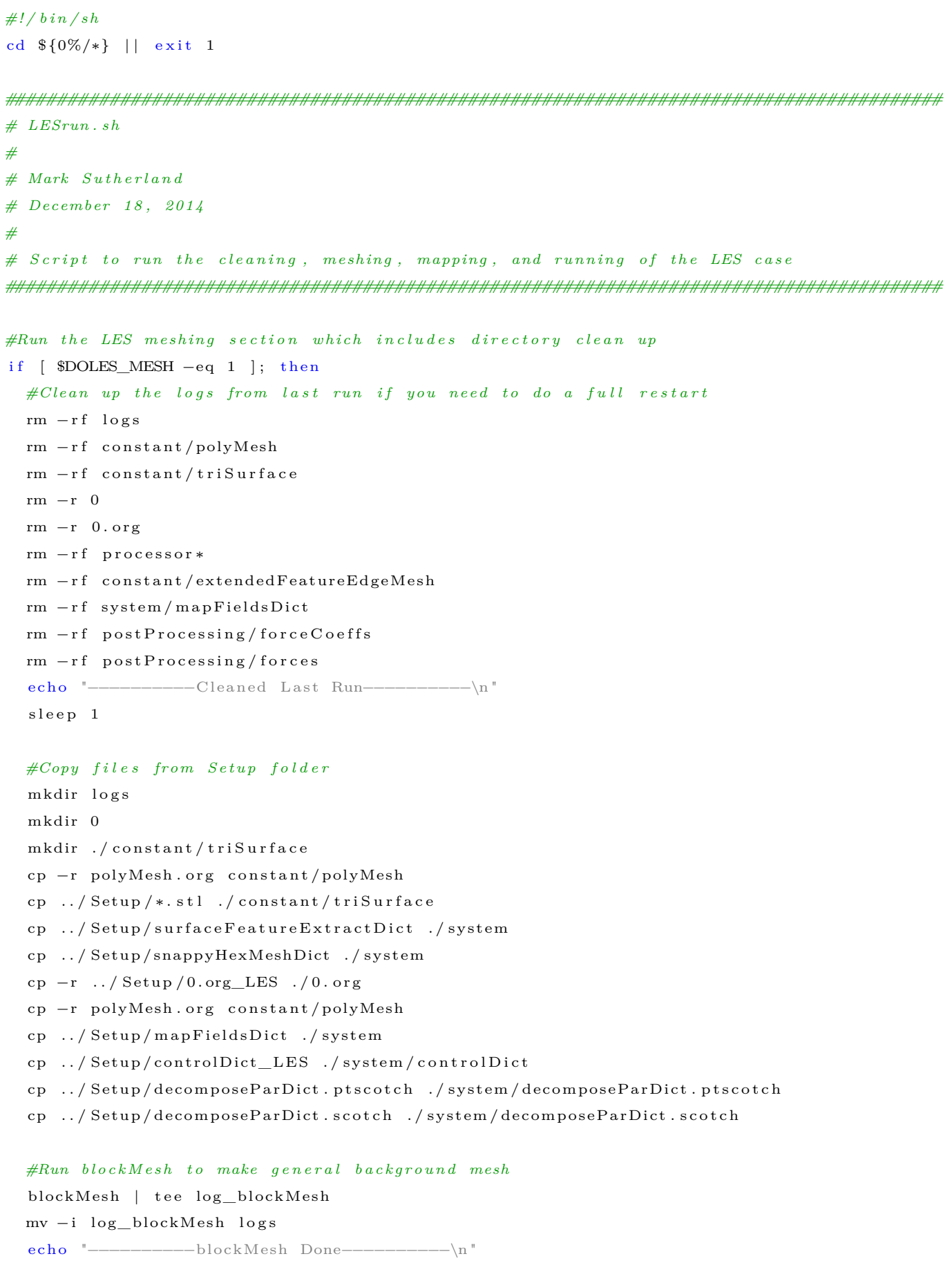




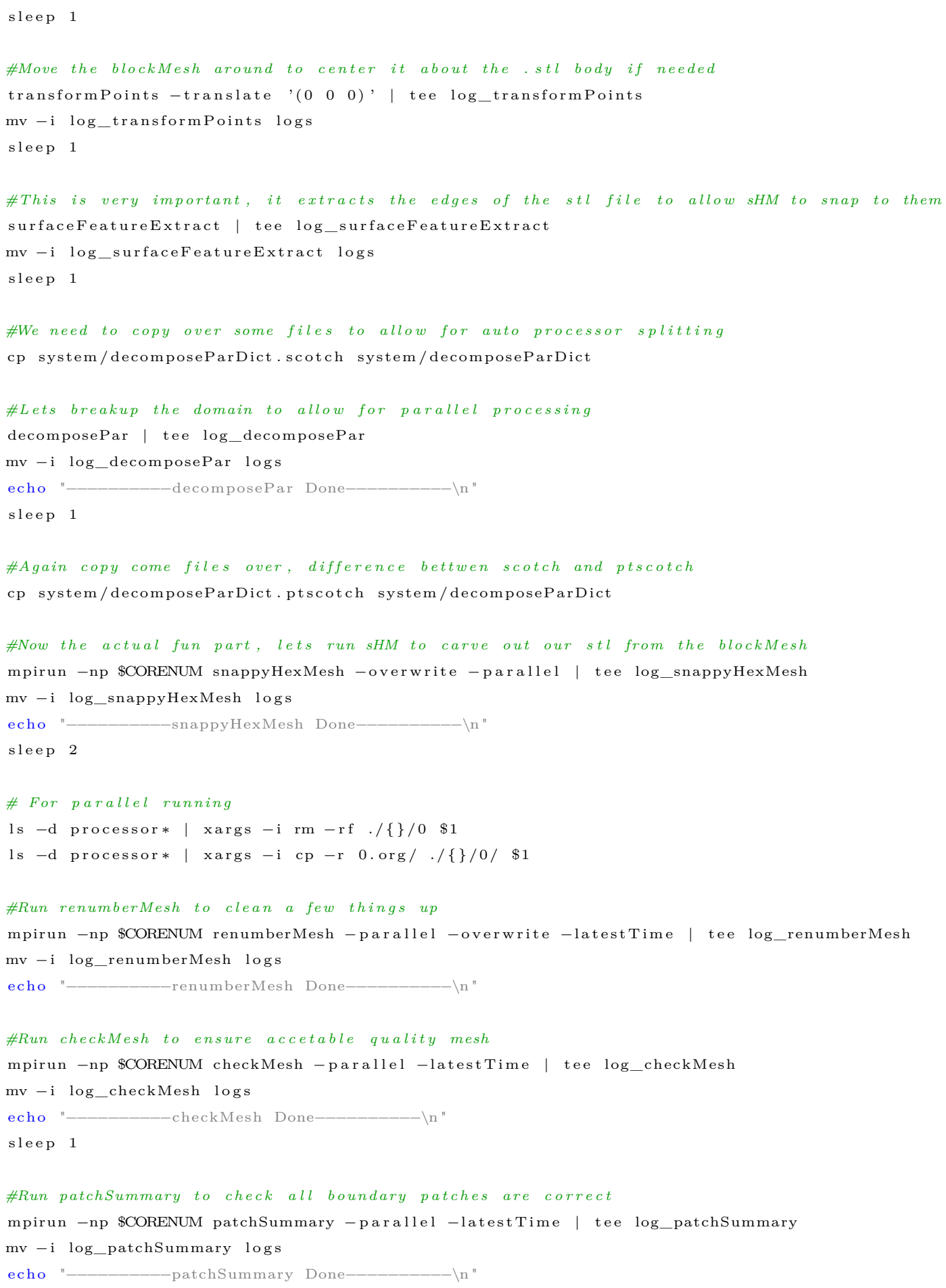




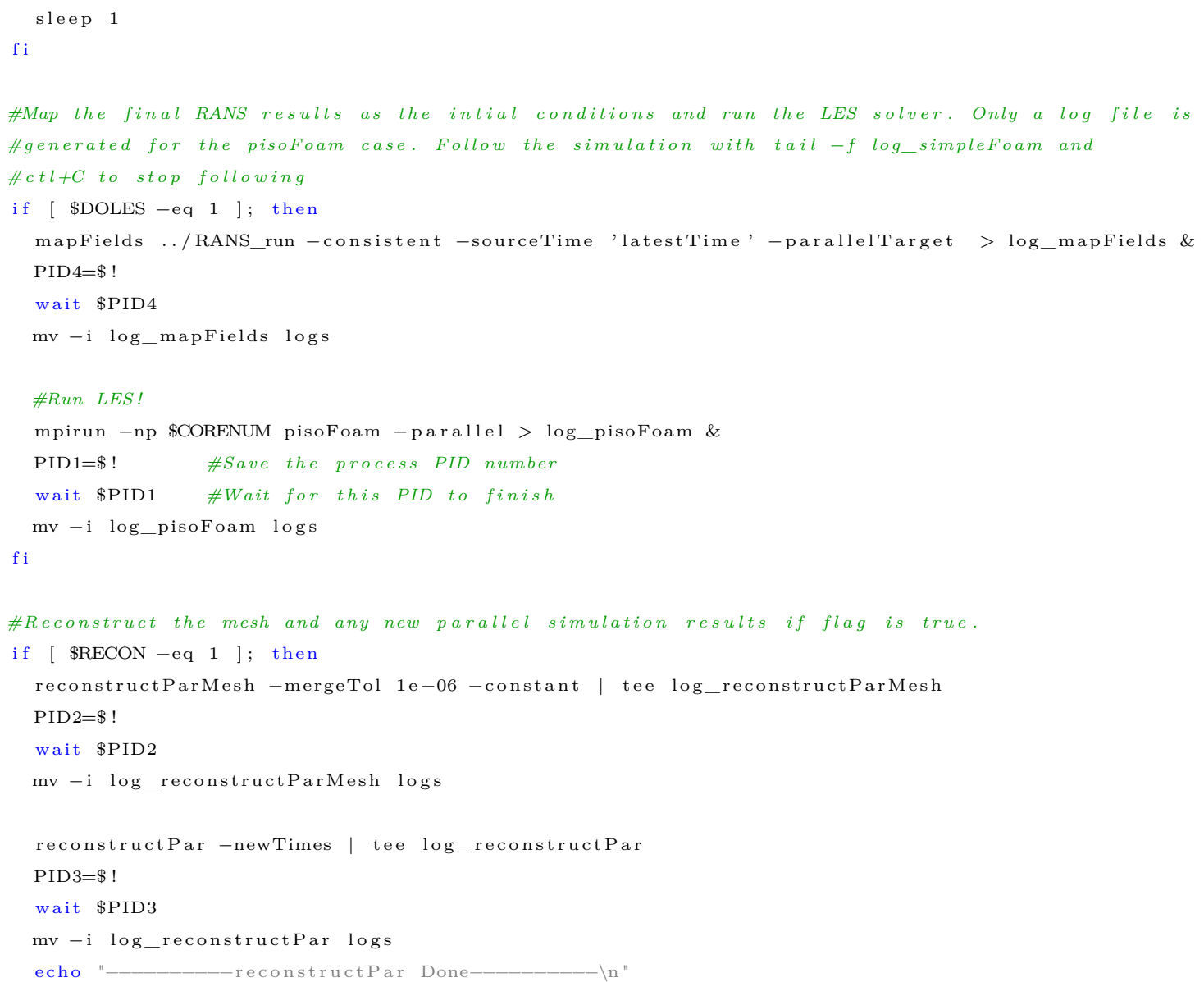




\section{Appendix B}

\section{Using OpenFOAM on Kumomotojo}

This appendix details how to use the outlined standard working directory (SWD) and remotely interface with the OpenFOAM/Paraview installation on the Kumomotojo workstation.

\section{B.1 Using OpenFOAM}

As outlined in Chapter 2 version 2.2.x of OpenFOAM is currently used to generate the urban wake fields, however Kumomotojo has both version 2.2.x and 2.3.x installed if the added features are required in the future ${ }^{1}$. To select a version, you must enter one of the following two alias commands whenever you open a new terminal.

of $22 \mathrm{x}$

This allows multiple versions of OpenFOAM to work along side one another and provide backwards compatibility for users with existing scripts. To test which version of OpenFOAM is active, if any, call for the help output of the transient laminar

\footnotetext{
${ }^{1}$ See http://www.openfoam.com/ for the change logs and details.
} 
incompressible flow solver with the following command,

$$
\text { icoFoam -help }
$$

If everything works as planned, the icoFoam usage output shown in Figure 57 should print to the terminal.

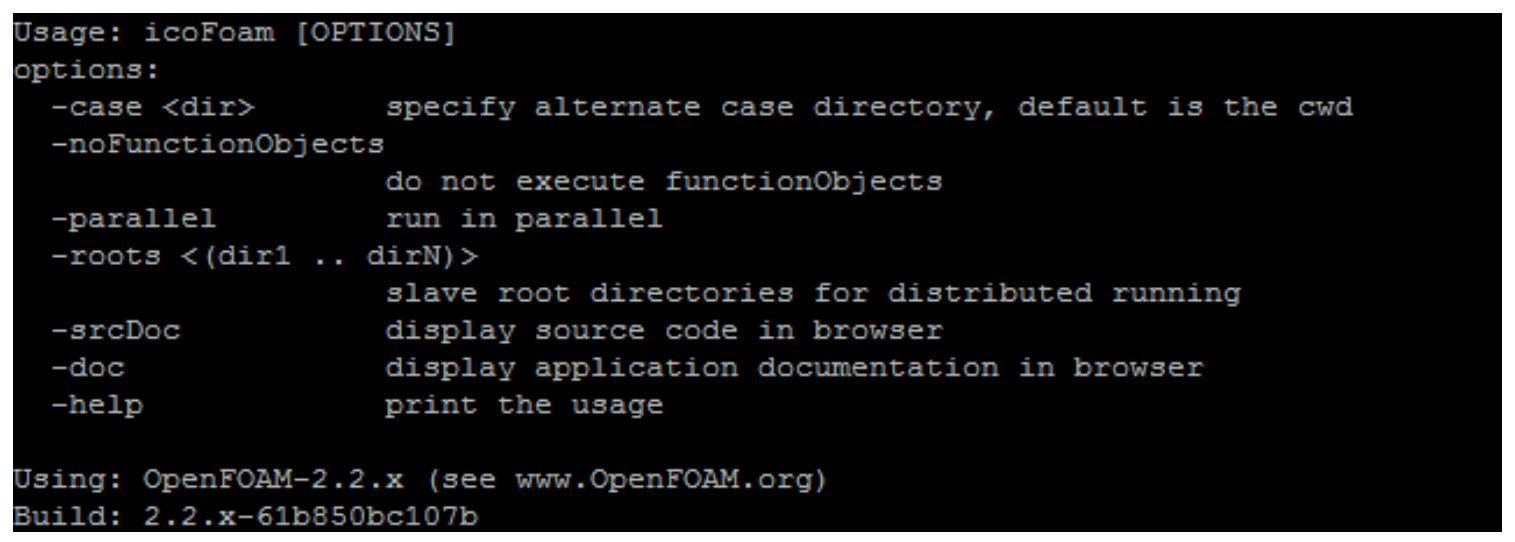

Figure 57: icoFoam usage output with OpenFOAM version

If an OpenFOAM function, such as icoFoam, is called or tab completion does not work a version has not been selected and the messages in Figure 58 will appear. This usually occurs in the haste of opening a new or second terminal window without entering a version selection command as previously outlined.

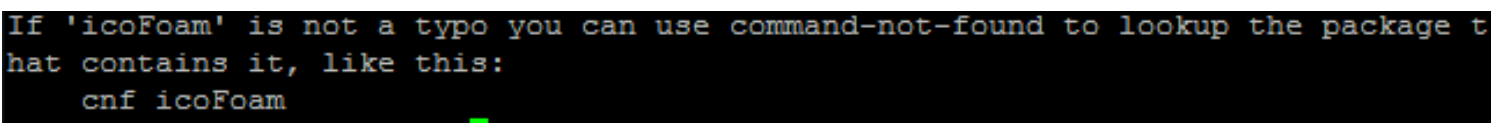

Figure 58: icoFoam function not found message 
The SWD should be downloaded, placed, renamed, and setup in the OpenFOAM run directory. This subfolder is in the OpenFOAM folder in the user's home directory, along with the OpenFOAM tutorials, and can easily be accessed with the following change directory alias,

\section{run}

Therefore when the SWD is prepared and ready, the simulation process is started with the following sequence of commands from a new terminal screen,

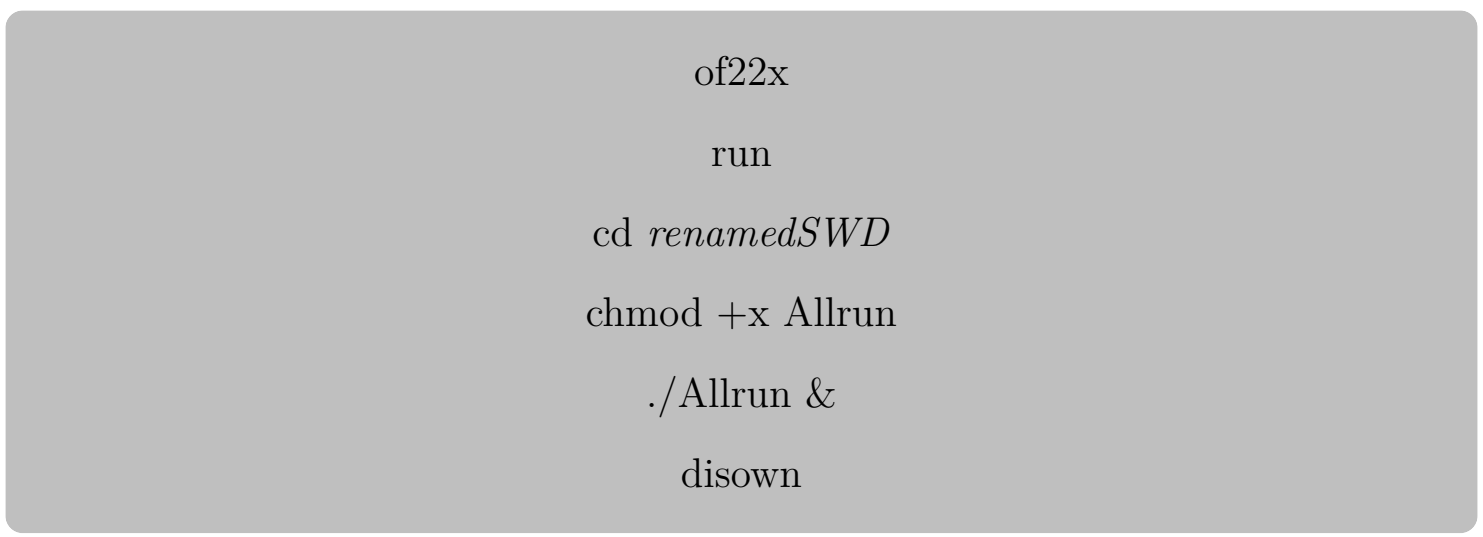

The \& and disown commands are important as they place the simulation in the background and then detach it from the current terminal respectively. After the ./Allrun \& command is entered, text will start to flow as the initial outputs of the scripts are teed to both a log file and the terminal screen. Simply typing disown and pressing enter 'in the text' will disown and detached the current processes allowing the user to close the terminal and/or logout. The top command can then be used to see what application is currently running and the log can be viewed in real time using the following command (in the directory of the log file), 


\section{tail -f log_applicationName}

This will produce a self scrolling output of the log file which can be exited at anytime pressing $c t l+c$. Similarly each case contain Residuals and Residuals_logs files which can be used to monitor the simulation residuals with gnuplot. The four files have been created to take into account the two solving algorithms, simpleFoam and pisoFoam, as well as the log file location, currently being written to or saved in the $\operatorname{logs} /$ folder. Therefore a desired command would take the form of and requires $\mathrm{X}$ forwarding to view the resulting plot,

\section{gnuplot Residuals}

\section{B.2 Using ParaView}

ParaView is an open-source, multi-platform data analysis and visualization application based on the Visualization Took Kit (VTK) devopled by Kitware Inc, and the Los Alamos and Sandia National Laboratories ${ }^{2}$. ParaView is used in conjunction with OpenFOAM to view the 3D simulation results. A strength of ParaView is its ability to not only process very large data sets and decomposed simulations, but do so in a distributed server-client model to take advantage of remote computational rendering power. Kumomotojo has been setup with a server ability and the following section will detail how to install ParaView on a personal client and connect to the server.

\footnotetext{
${ }^{2}$ http://www . paraview.org/
} 
Currently ParaView 3.12 (for OpenFOAM-2.2.x) and ParaView 4.1.0 (for OpenFOAM-2.3.x) are installed on the server and for remote viewing the ParaView version on both the server and client must be the same. The various release builds of ParaView for all operating systems can be found here, http://www.paraview.org/download/, and should be downloaded and installed on the client computer. Linux users can look into their repository versions but it could prove easier to download or build from source.

ParaView has been designed to connect a client instance to a host server, rather than simply $\mathrm{X}$-forwarding the servers display. While $\mathrm{X}$-forwarding is far simpler to implement, the resulting performance is quite low and unstable. Therefore a SSH tunnel is used to forward the data from the server to the client via port forwarding.

\section{B.2.1 Configure Linux Client}

For a Linux based client the following alias can be added to the .bashrc file to simplify the tunnel startup,

\section{alias pfTunnel="ssh usr@XXX.XXX.XX.XX -L 11150:localhost:11115 -g -C"}

Where usr is replaced with your Kumomotojo login user name, and XXX.XXX.XX.XX with Kumomotojo's IP address. Port 11115 is the address the ParaView server will be publishing data to, based on the setup described below. Port 11150 represents the mapped client port which is 'connected' to the server point. Either port numbers can be changed, and should be for multi-connections 
and multi-users ${ }^{3}$, depending on the users existing port maps.

\section{B.2.2 Configure Windows Client}

For Windows based clients, Putty can be used to setup the SSH tunnel and port forward, found at http://www.chiark.greenend.org.uk/ sgtatham/putty/. Open an instance of Putty, and click Tunnels under the $S S H$ category, shown in Figure 59. Enter the source port, the IP address with ParaView server port, and click Add.

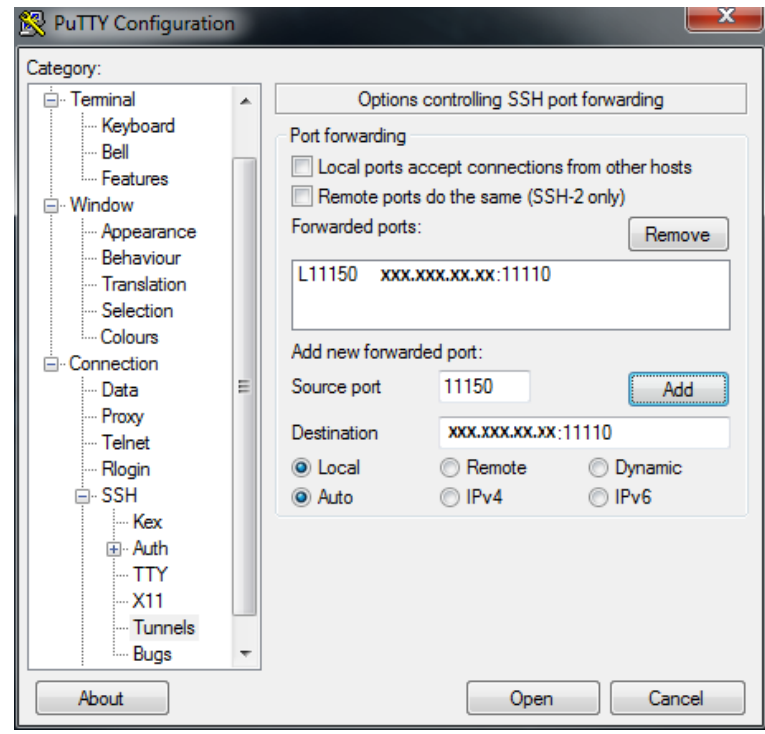

Figure 59: SSH tunnel Putty setup

Select the Session category, enter Kumomotojo's IP address, a desired name such as Kumo, and Save shown in Figure 60. This will save the IP address and SSH tunnel information for future connections by selecting the session name and selecting Load. Start the SSH session by clicking the Open button.

\footnotetext{
${ }^{3}$ Multi-connections and multi-users are untested and tests are recommended for future work
} 


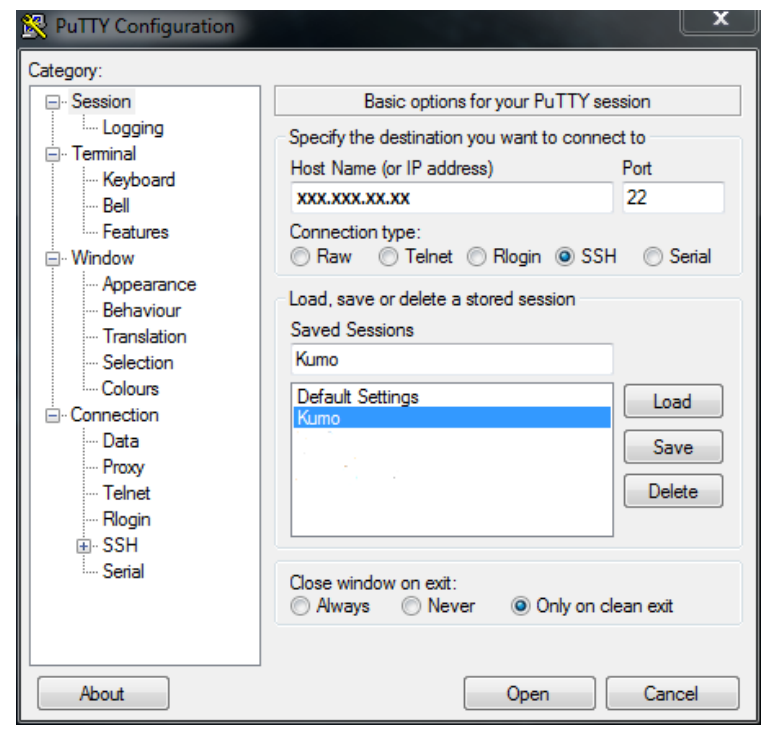

Figure 60: Putty connection setup

With the SSH tunnel setup between the server and client, the next step is to start the ParaViw Server. To facilitate easy startup times, the following aliases should be added to your users .bashrc file on the server,

$$
\begin{aligned}
& \text { alias pfServer }=\text { "pvserver }- \text { server-port }=11115 " \\
& \text { alias pfServer_OS="pvserver }- \text { server-port }=11115-\text { use-offscreen-rendering" } \\
& \text { alias pfServer_M2="mpirun -np } 2 \text { pvserver }- \text { server-port }=11115 " \\
& \text { alias pfServer_M4="mpirun -np } 4 \text { pvserver }- \text { server-port }=11115 "
\end{aligned}
$$

These sets of commands are used to start an instance of a ParaView server with different settings. There are 3 main options for the server: 


\section{- pfServer}

- Runs a 'standard' pvserver such that is uses the GPU to render into an X window before showing it in the GUI. This window will pop open on the client's desktop automatically when the system needs to render something, such as a coloured contour plane. This is a single serial server meaning the size of mesh/simulation is limited. The upper limit is not clearly defined but a rough estimate would be my course LES grid with $1.13 \times 10^{6}$ cells. If the server connection drops or takes an excessively long time to load, the server needs more power by running in one of the parallel modes outlined below.

\section{- pfServer_OS}

- If the server does not have GPU rendering capabilities with OpenGL, the ParaView server uses CPU based rendering though off screen Messa (MessaOS) libraries. This option can also be used if there is an issue with the client opening $\mathrm{X}$ windows on the desktop. Since the rendering is being performed by the CPU's, which are typically already busy running simulations, the simulation size is even more limited than the pfServer option. While the response time is slightly quicker than rendering with an X window, only simple simulations of no more than 500, 000 cells should be opened with the server in this mode.

\section{- pfServer_M2 and pfServer_M4}

- For large simulations, such as the Medium and Fine LES simulations $\left(2.43 \times 10^{6}\right.$ and $7.67 \times 10^{6}$ cells $)$ the server should be run in a parallel 
mode to distribute the load. The parallelism of the ParaView server is only limited by the hardware of the server therefore these two commands serve only as shortcuts. Even 4 processors should be more than enough to run a server any simulations run on Kumo, however is the number of processes can easily be changed by entering the raw terminal command. While it does employ GPU rendering, running the server in parallel will make a thread for each server section thereby taxing the 'usually always in use' CPUs. pfServer_M4 should only be used for short periods of post processing if all CPUs are busy to prevent automatic thermal shutdowns of the server.

With the SSH tunnel and ParaView server setup, the last item is to configure ParaView on the client side. After the appropriate version has been downloaded and installed, multiple ParaView versions can be installed on the client, open a new instance of ParaView and select the connect to server button, circled in Figure 61. 


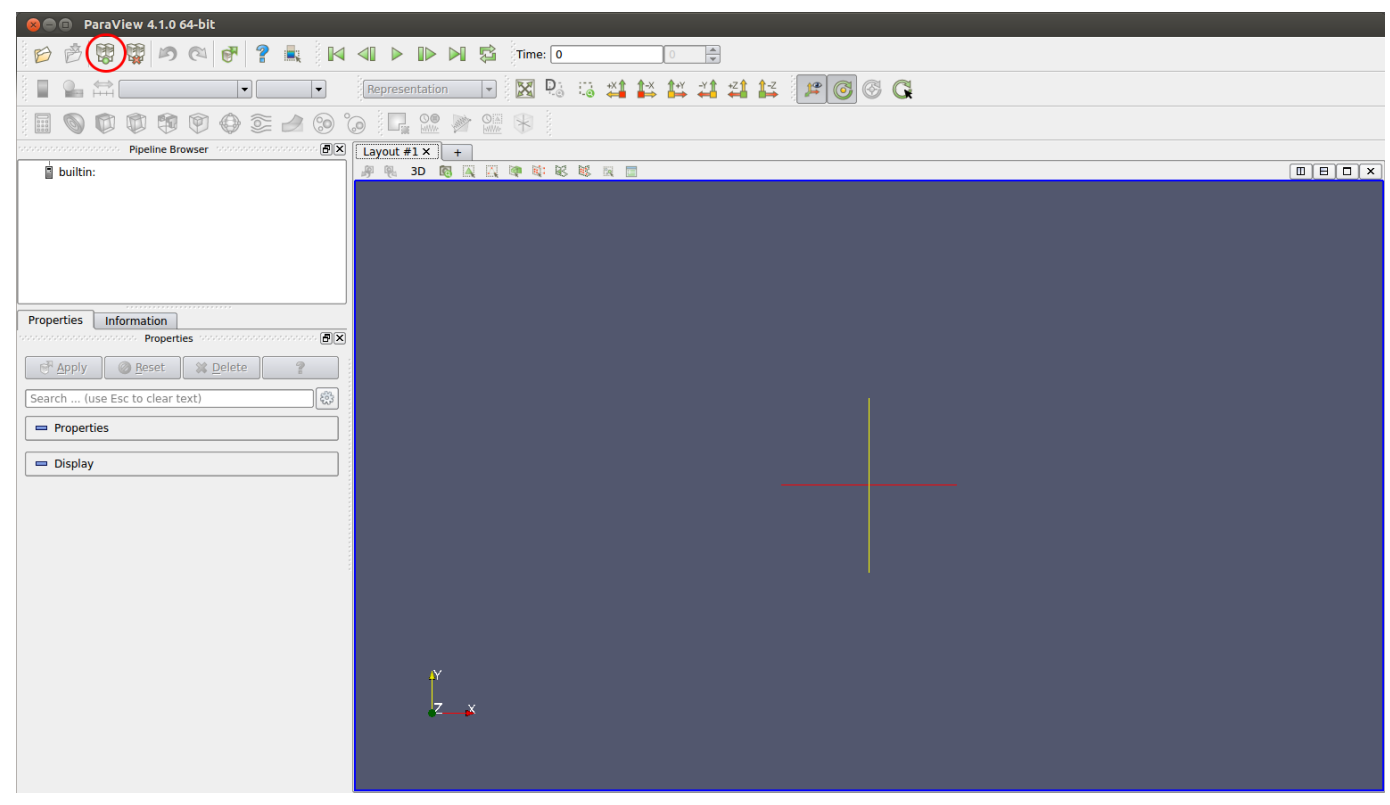

Figure 61: Connect to server selection

In the pop-up window select Add Server, shown in Figure 62.

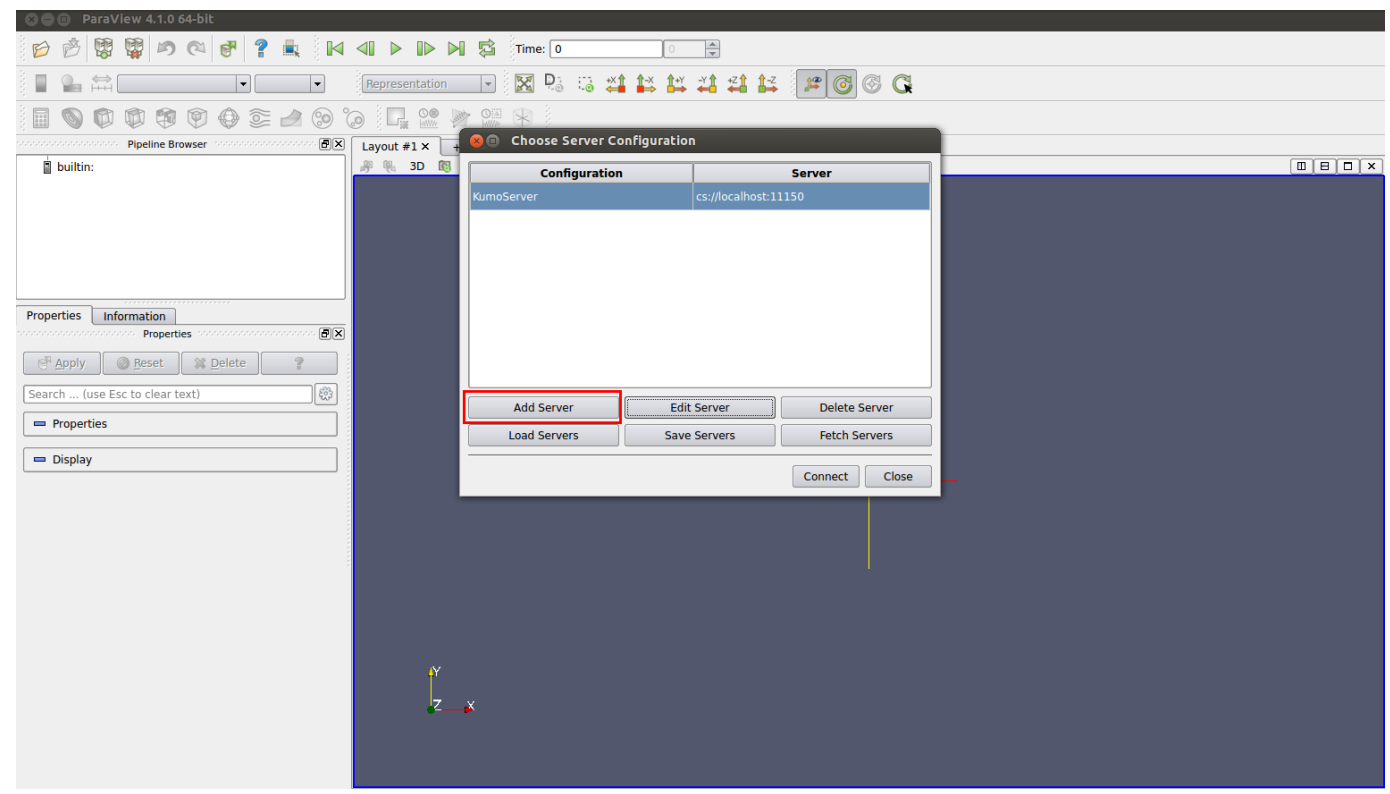

Figure 62: Add new server 
Choose a name, leave localhost and enter local forwarded port.

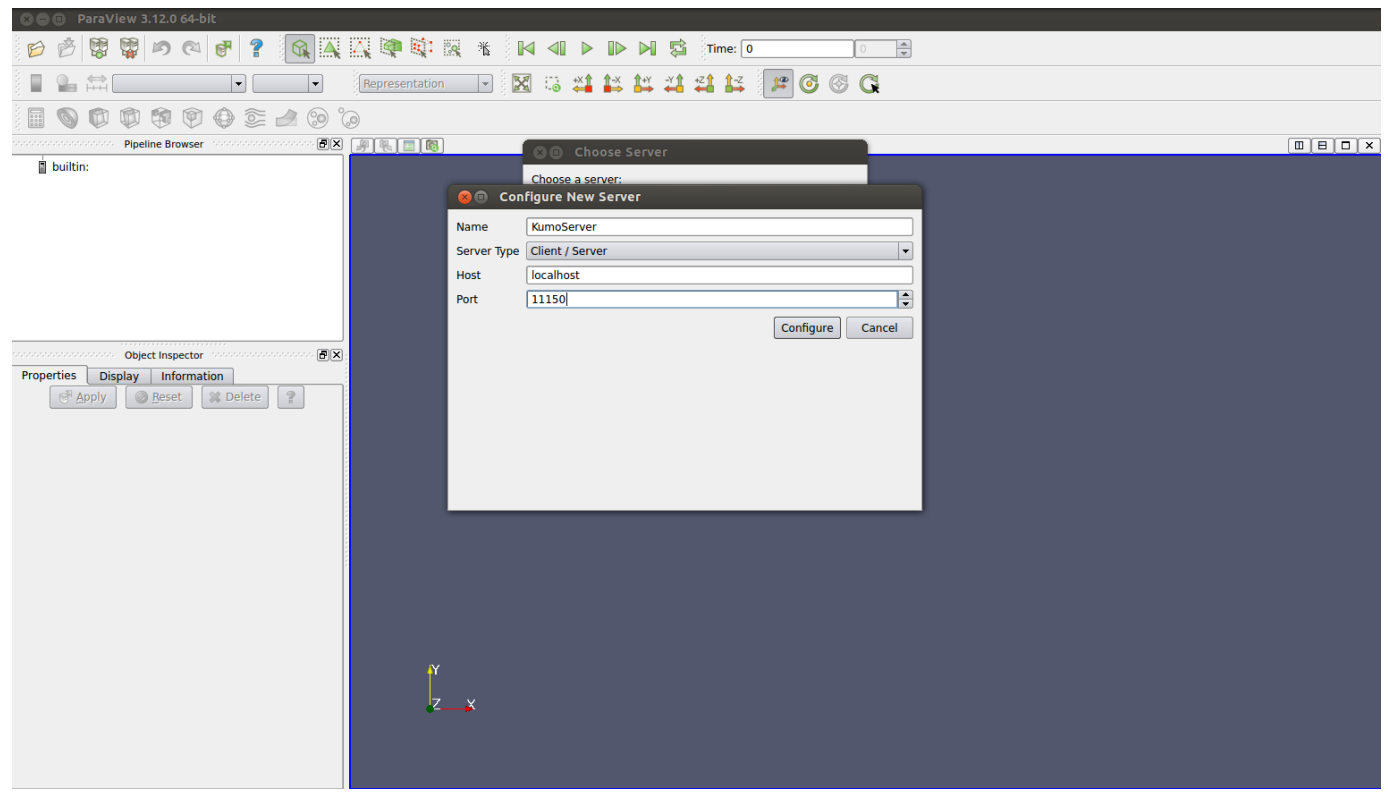

Figure 63: Enter the server details

Select Manual, meaning we will start the server ourselves each time, and Save.

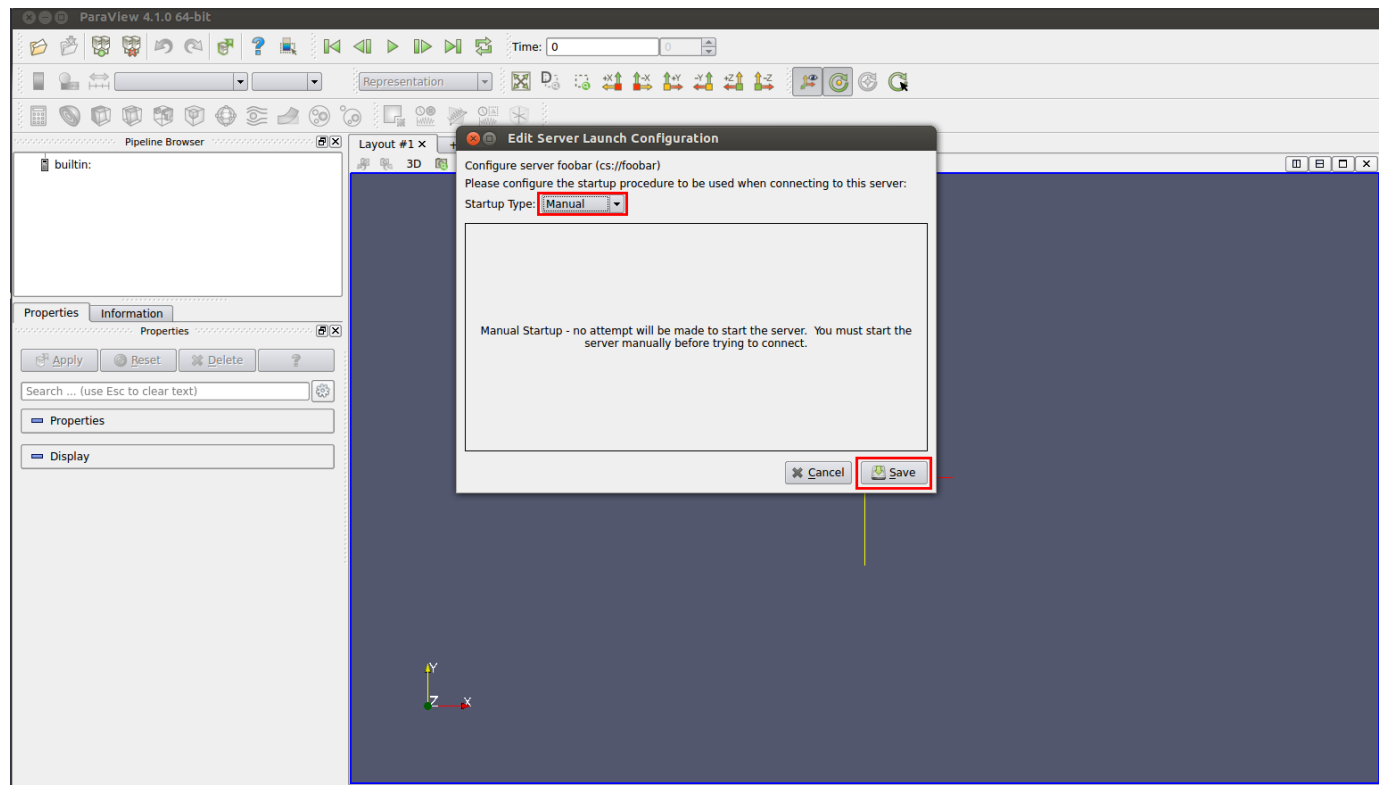

Figure 64: Select Manual and Save 


\section{B.2.3 Results Viewing}

ParaView should now be setup and ready to post-process the OpenFOAM simulation results. To start viewing data remotely either; open a new terminal and enter pfTunnel, or open Putty and load the saved Kumo configuration, if the client is Linux or Windows respectively. You should be prompted for your Kumomotojo user name and/or password. Select the version of OpenFOAM you wish to use/used by entering one of the alias commands, of22 $x$ or of23x.

Navigate to the foam.run file in the desired simulation case, however this is optional as you can browse to your case through the client GUI later. Start a ParaView server by entering one of the server start aliases, pfServer, pfServer_OS, pfServer_M2 or pfServer_M4, or a custom parallel server though the mpirun command. Open an instance of ParaView on the client and select the connect to server button on the top toolbar, Figure 65.

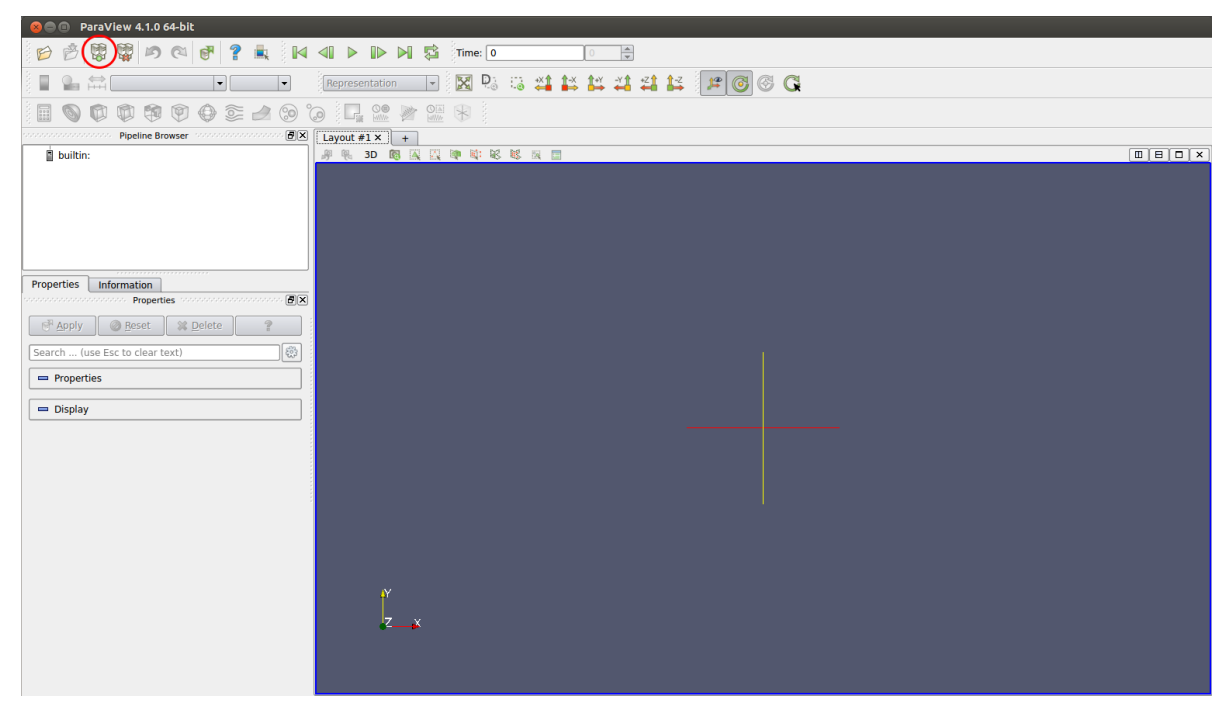

Figure 65: Connect to server selection 
When the Choose Server dialog box pops up, click the name of the server from Figure 66 and select connect. You can check the connection status by looking at the SSH tunnel terminal window which should say "Client connected".

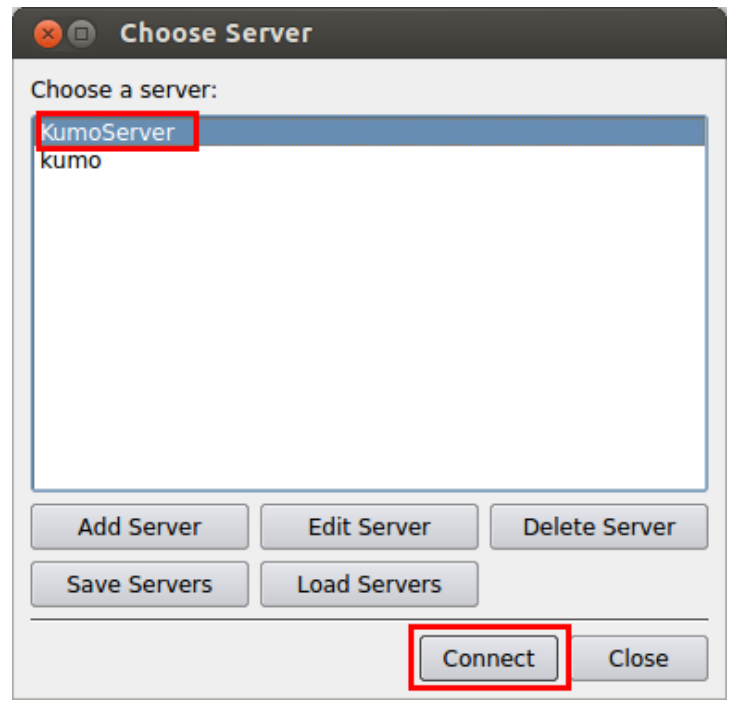

Figure 66: Server selection

If everything is connected correctly you should see cs://localhost:11150 under the Pipeline Browser (of a different number if you forward to a different port). To open a case press the open button, left most on the top toolbar, shown in Figure 67. The directory it opens to will be the directory where you started the server. Navigate to your case's run.foam file and open. Select either Reconstructed or Decomposed under case type and press the green Apply button. It can take a minute or so to load the data and open the rendering $\mathrm{X}$ windows if needed. The simulation results can now be remotely viewed. A graphical illustration of this example workflow is shown in Figure 68. 


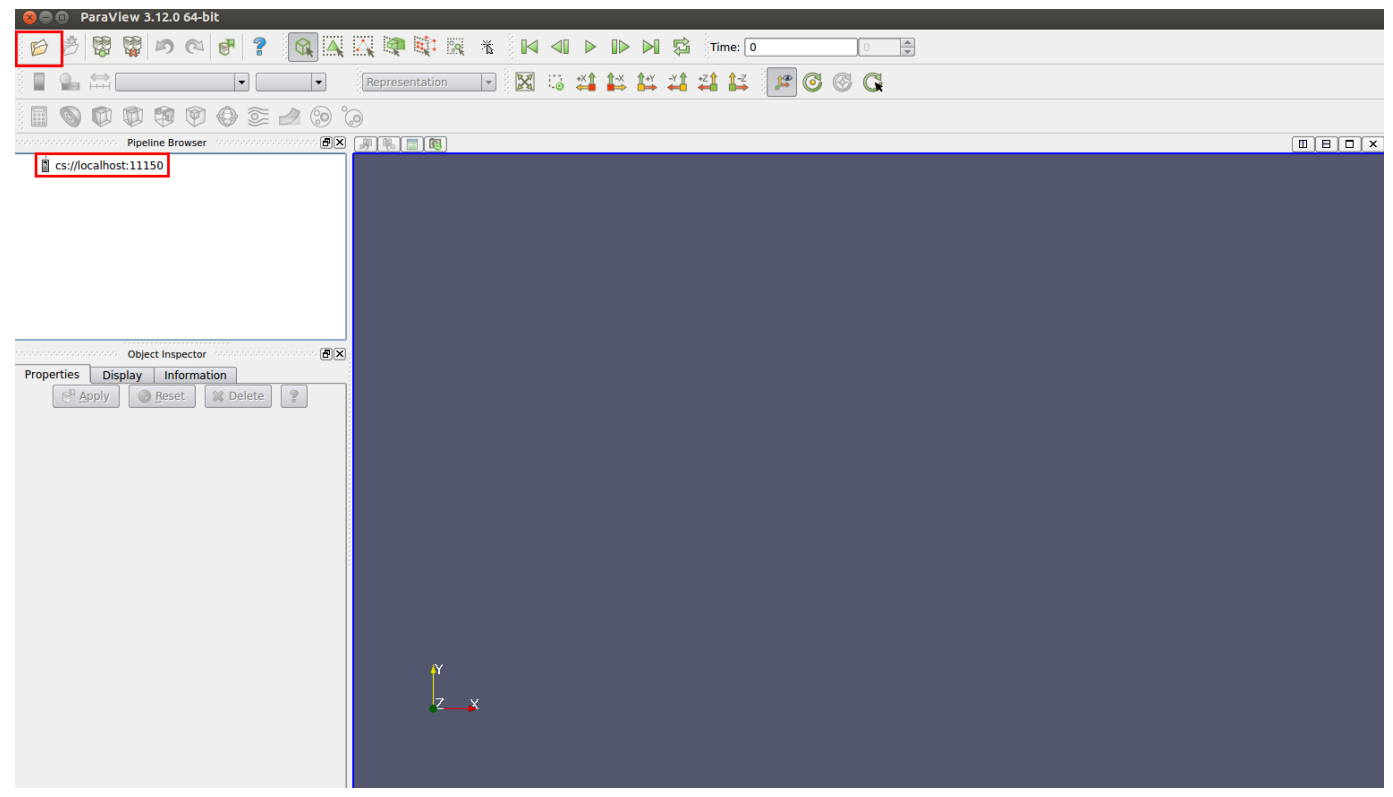

Figure 67: Server connection and open a case

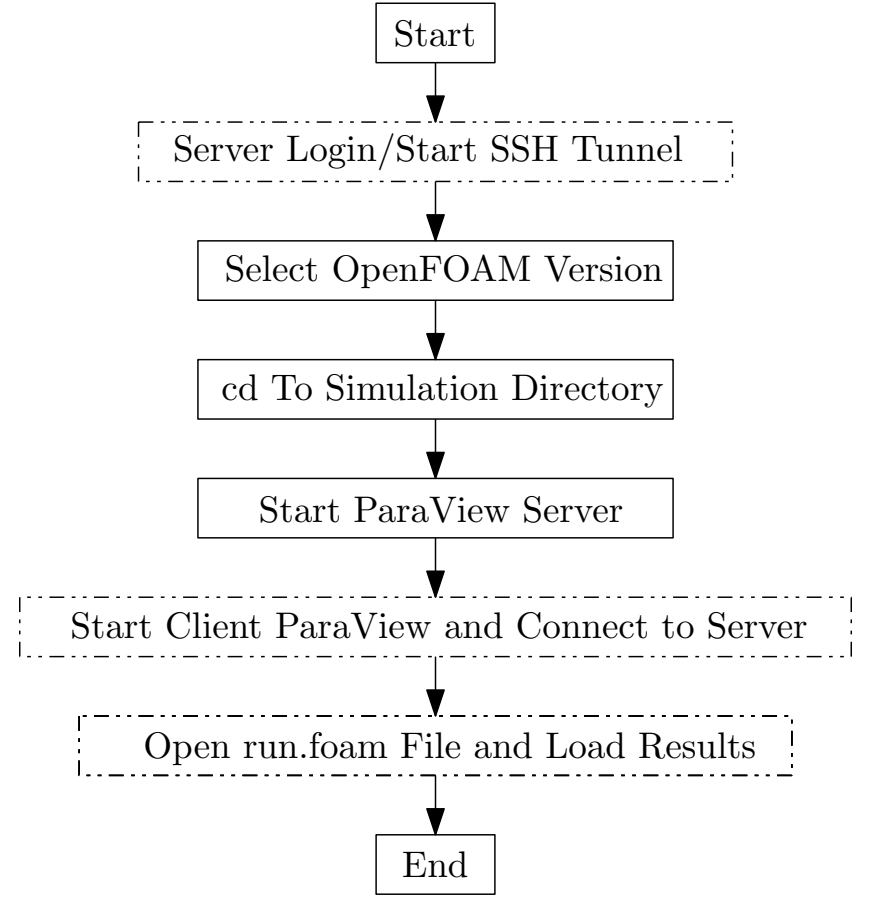

Figure 68: Remote ParaView workflow, dashed: client actions, solid: server actions 


\section{Appendix C}

\section{Urban Wake Database}

This appendix details how the results of the OpenFOAM LES are post-processed into .csv files and how those files are further processed and uploaded to the urban wind database. To first export the .csv files, open the desired LES run.foam file with ParaView and ensure the internalMesh box is checked after the loading. Forward to the last timestep by selecting the top circled button in Figure 69. This is required as only the $\mathbf{U}$ and $\mathbf{p}$ fields should be saved to the .csv files and additional variables are generated which are not present at the start of the simulation, such as U_Mean, or Q. Therefore ensure only $\mathbf{U}$ and $\mathbf{p}$ are selected under Cell Arrays, circled in Figure 69, and make sure to click the green Apply button.

From the file menu select Save Data and the Save File dialog will open. Enter the desired prefix, following the database convention, and ensure to leave an underscore at end, as illustrated in Figure 70. Each timestep is saved a separate .csv file starting from zero and the number is appended to the entered prefix. This format is required for the renaming portion of the post-processing scripts introduced below. 


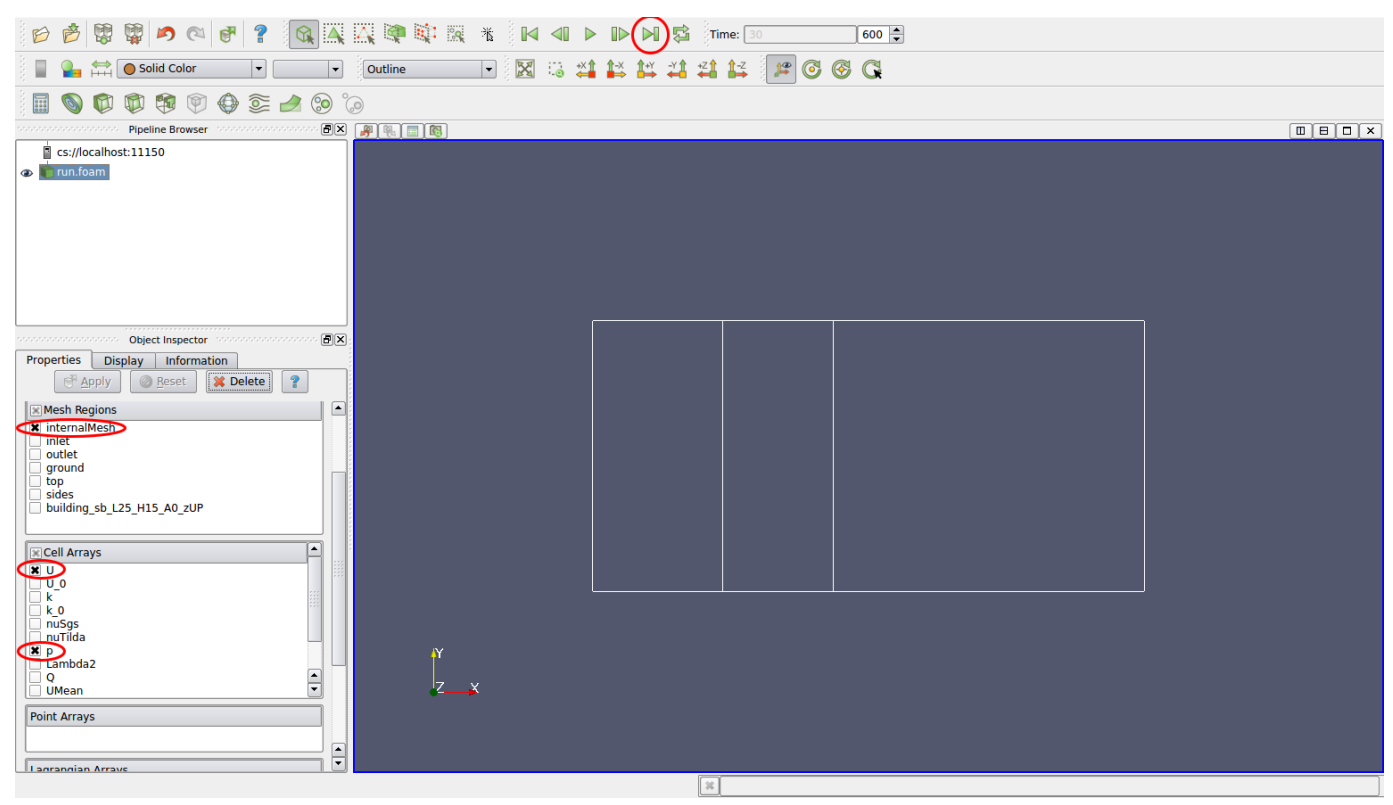

Figure 69: Load run.foam file, adjust timestep and select desired variables

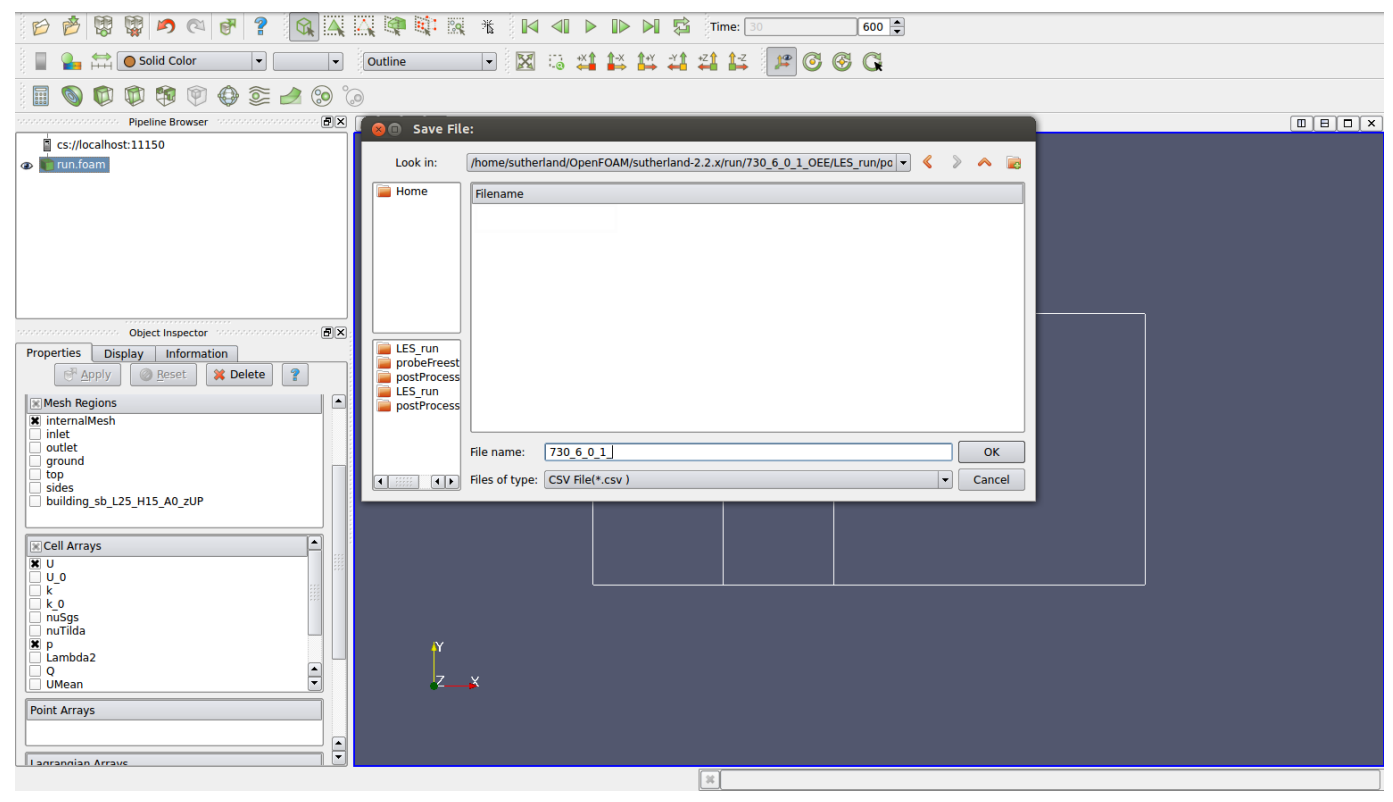

Figure 70: Save data and set file prefix 
Configure the writer to save all the timesteps and ensure the field is association on the points, shown in Figure 71. After clicking $O k$ it will take some time to generate the .csv files, a function of the write interval and total simulation time. Similarly, the files can take on the order of $40 \mathrm{~GB}$ of hard drive space prior to trimming so ensure sufficient storage space is available.

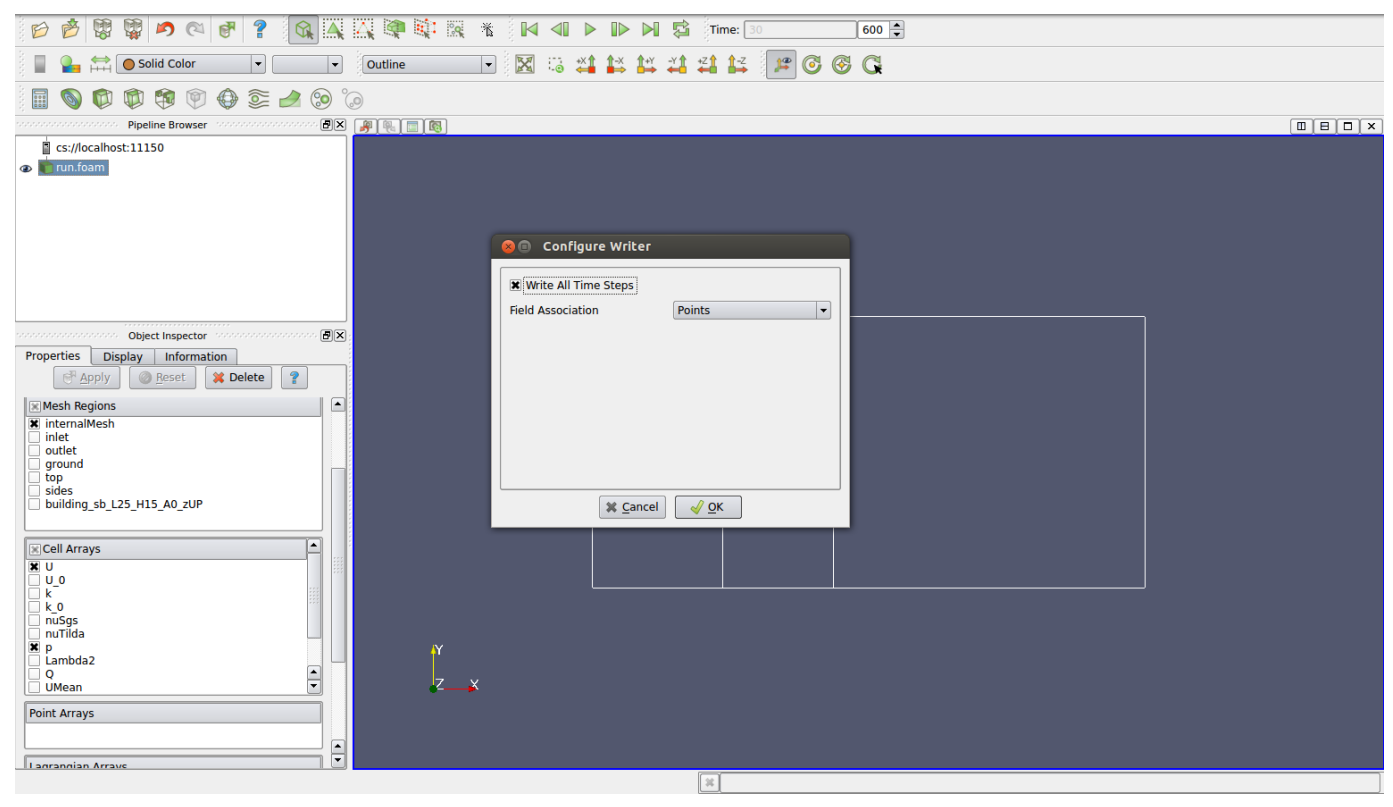

Figure 71: Save the data from the points and for all timesteps

After all .csv files have been generated they are trimmed and processed using the extractData.py and csvImport.sh scripts in the postProcessing sub folder. The python extractData.py script is used to trim each .csv file in space and time, and rename/renumber the remaining files. The commented script is shown in Listing C.1 with details on what should be mortified before execution. After modifying the script with; the fileName, start and end loop times, and desired wake region, the trimming is performed by entering python extractData.py form the command line. 
Listing C.1: extractData.py

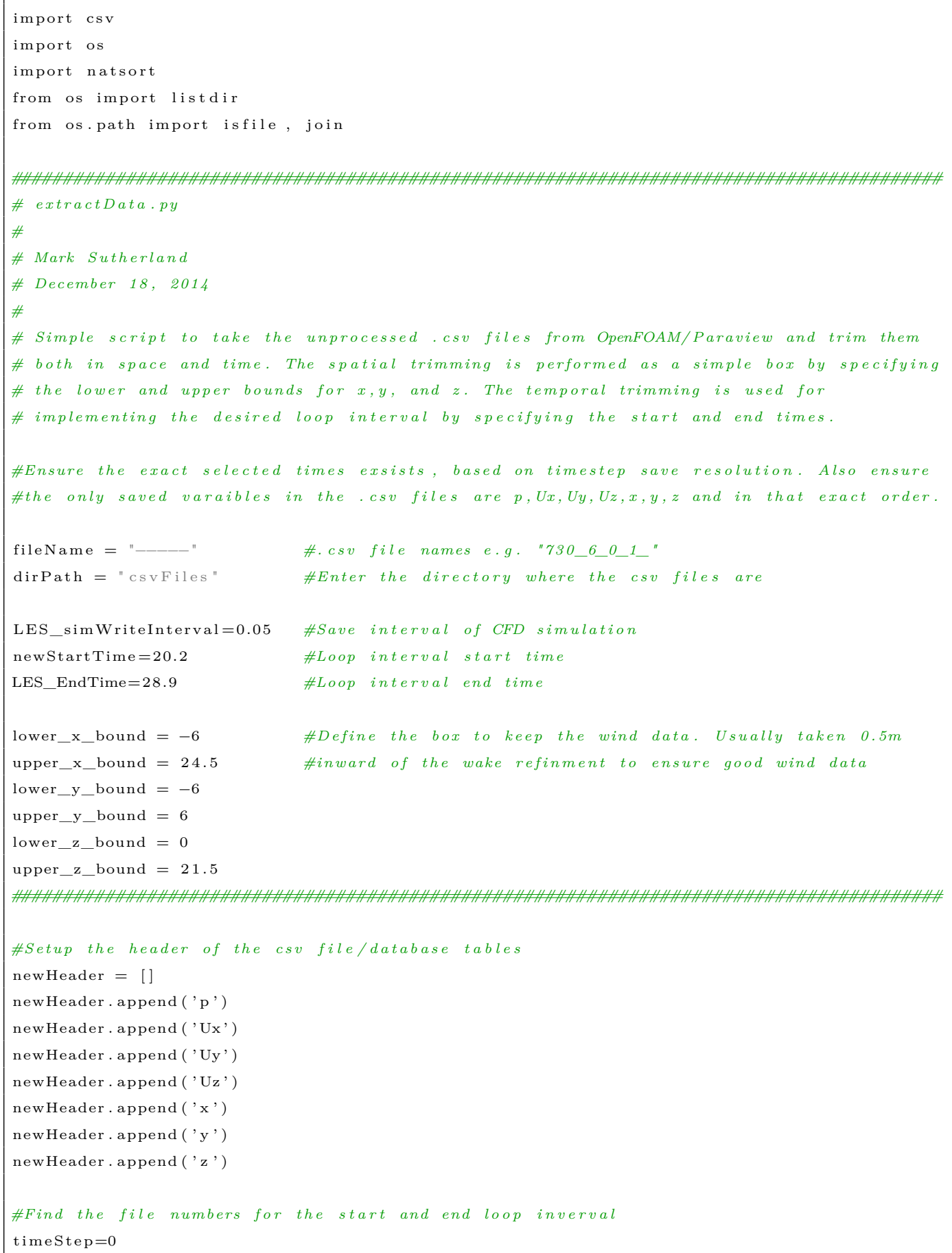




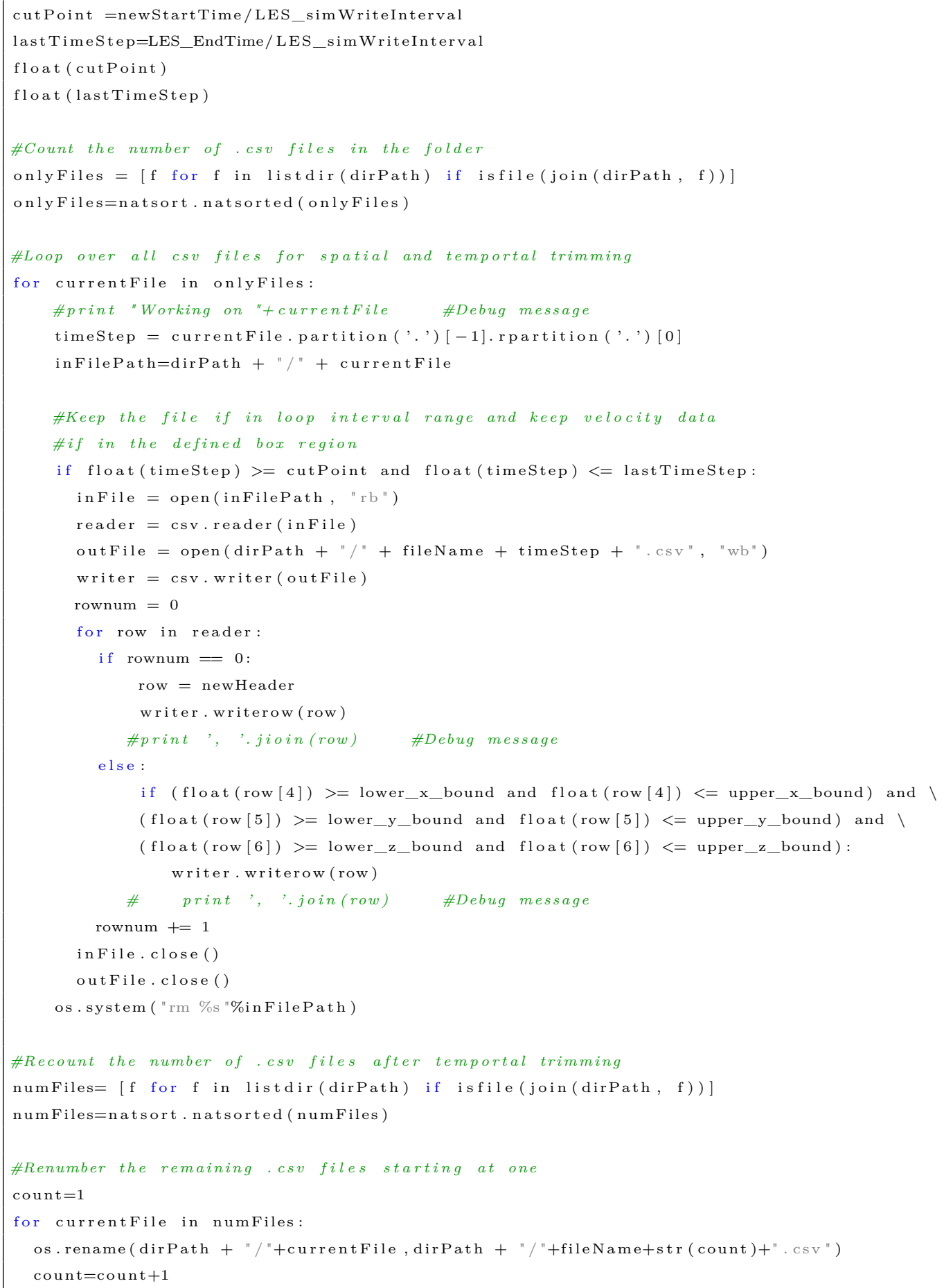


\#Script end message

print "Exraction Finished"

Once the .csv files have been trimmed they can be uploaded to the MySQL based Urban Wind Database using the csvImport.sh bash script, shown in Listing C.2. After editing the file for the database name, user name, user password ${ }^{1}$, and base filename the script is run from the command line using ./csvImport. If required, make the file executable by entering chmod $+x$ csvImport. The script will go through each .csv file, make a new table for that timestep and load the data into the database. One important note are the saved variables and subsequently the database headers. The variables and their order are manual configured in both scripts. Ensure the number and order and uniform across the exported .csv files, extractData.py script and csvImport.sh script.

Listing C.2: csvImport.sh

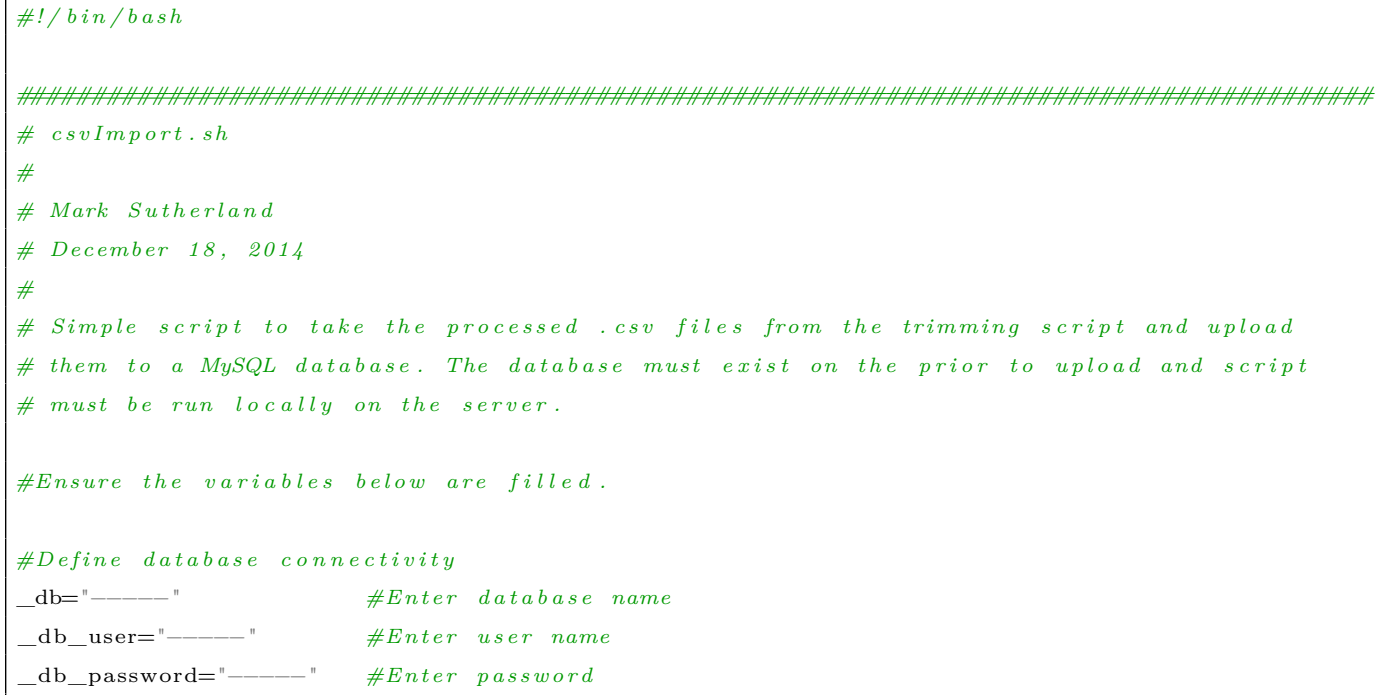

${ }^{1}$ The user names and passwords for the Kumomotojo database are removed due to the public availability of the scripts. 


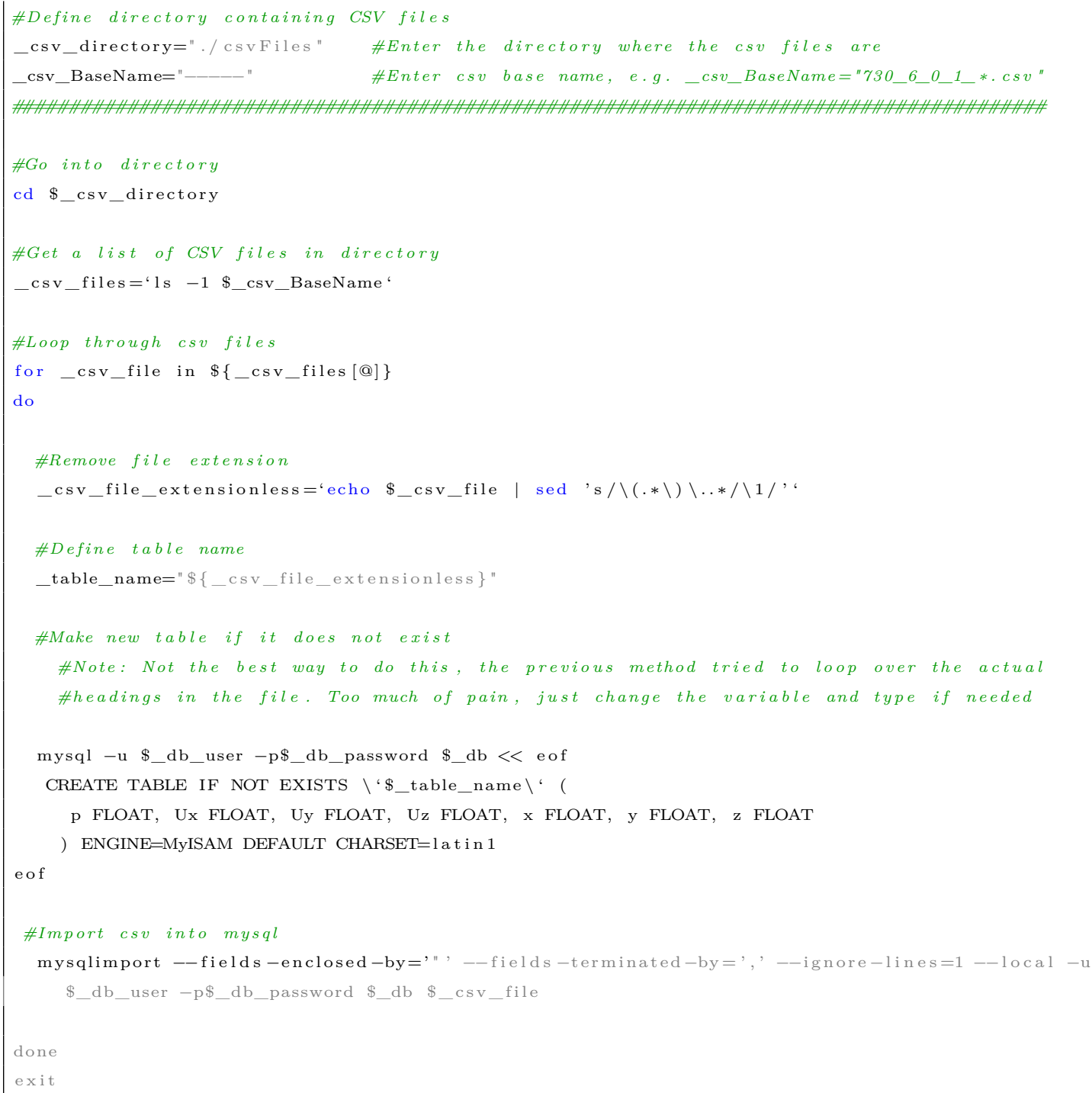




\section{Appendix D}

\section{Database-Simulator Integration}

This appendix details how to remotely connect to the MySQL urban wind database for use with the Tara MATLAB flight simulator. Additionally, the naming convention of storing the CFD results and basic MySQL commands to interact with the database are provided. For the MATLAB/Simulink flight simulator to work the following toolboxes must be installed

- Aerospace Blockset

- Aerospace Toolbox

- Database Toolbox

- Fuzzy Logic Toolbox

- Model Predictive Control Toolbox

- Neural Network Toolbox

To see a complete list of the installed toolboxes, open MATLAB and use the ver command in the Command Window. Similar to the other Appendices, placeholders are used for sensitive details such as IP addresses, user names, and passwords which will be available as needed. 


\section{D.1 MySQL Database Structure}

As outlined in Chapter 1, only a single building in isolation is used as an initial test of the quadrotor's performance with LES based wake fields. However, the urban wind database (UWD) currently contains both the RANS and LES generated wake field used in the results chapter. The database table naming follow a similar convention laid out by Galway [13] and are based on the defining parameters of the building, defined in Figure 7a and shown again in Figure 72.
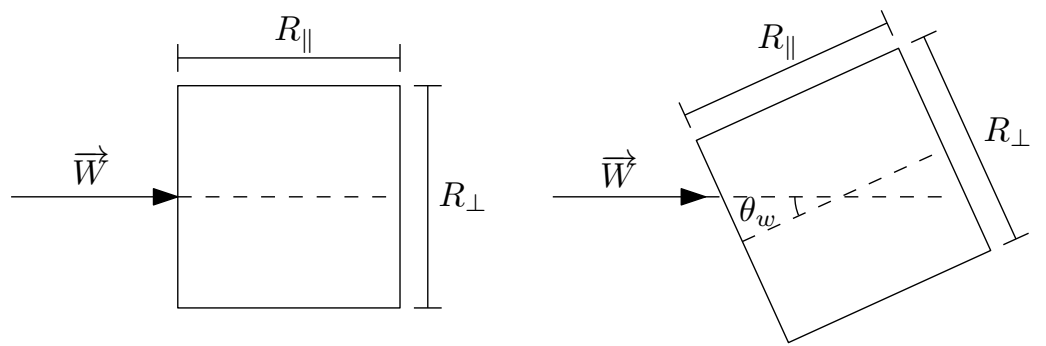

Figure 72: Single building characteristics

Therefore each database table takes the form of,

$$
\operatorname{Re}_{B \_} \operatorname{Re}_{E \_} \theta_{w \_} R_{\perp} / R_{\|} \mathrm{T}
$$

Where:

- $\operatorname{Re}_{B}$ is the base of the Reynolds Number

- $\operatorname{Re}_{E}$ is the exponent of the Reynolds Number

- $\theta_{w}$ is the wind angle

- $R_{\perp} / R_{\|}$is the ratio of the building's side lengths

- $\mathrm{T}$ is the CFD simulation timestep number

For example, the 42nd timestep from the results used in this thesis would be 730_6_0_1_42 for the LES case and 730_6_0_1_RANS_42 for the RANS. 


\section{D.2 SSH Tunnel}

Similar to the remote ParaView, a SSH tunnel is used to bridge the local flight simulator with the MySQL database ${ }^{1}$. This section will detail how to setup the SSH tunnel on Linux and Windows based clients.

\section{D.2.1 Configure Linux Client}

For a Linux based client the following alias can be added to the .bashrc file to simplify the tunnel startup,

\section{alias uwdTunnel="ssh -f usr@XXX.XXX.XX.XX -L 7800:XXX.XXX.XX.XX:3306 -N"}

Where usr is replaced with the UWD user name for the Kumomotojo server, and XXX.XXX.XX.XX os Kumomotojo's IP address. Port 3306 is the normal MySQL address and will be mapped to port 7800, the latter can be changed depending on the users existing port maps ${ }^{2}$. The flags $-\mathrm{f},-\mathrm{N}$, and -L are used for; running ssh in the background, tells ssh to not enter any commands once the tunnel is open, and sets up an ssh tunnel that connects port 7800 on the localhost to port 3306 (default MySQL port) on the server respectively.

\section{D.2.2 Configure Windows Client}

For Windows based clients, Putty can be used to setup the SSH tunnel and port forward, found at http://www.chiark.greenend.org.uk/ sgtatham/putty/. Open

\footnotetext{
${ }^{1}$ Remote running of the simulator is future work

${ }^{2}$ Multi-connections, multi-users, and different port numbers are for future work
} 
an instance of Putty, and click Tunnels under the SSH category, shown in Figure 73. Enter the source port, the IP address with MySQL server port, and click $A d d$.

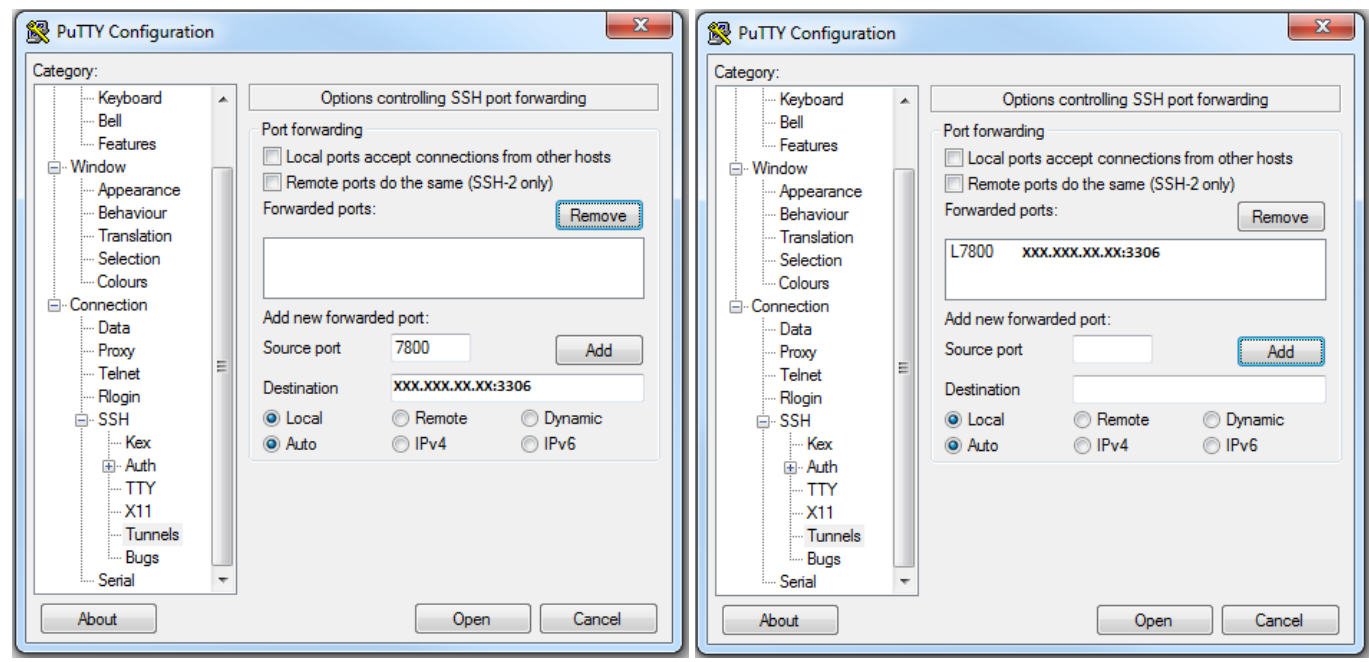

Figure 73: SSH tunnel Putty setup

Select the Session category, enter Kumomotojo's IP address, a desired name such as windDB, and Save shown in Figure 74. This will save the IP address and SSH tunnel information for future connections by selecting the session name and selecting Load. Start the SSH session by clicking the Open button ${ }^{3}$.

\footnotetext{
${ }^{3}$ Both tunnels for the remote ParaView and the database can be added to the same session/name
} 


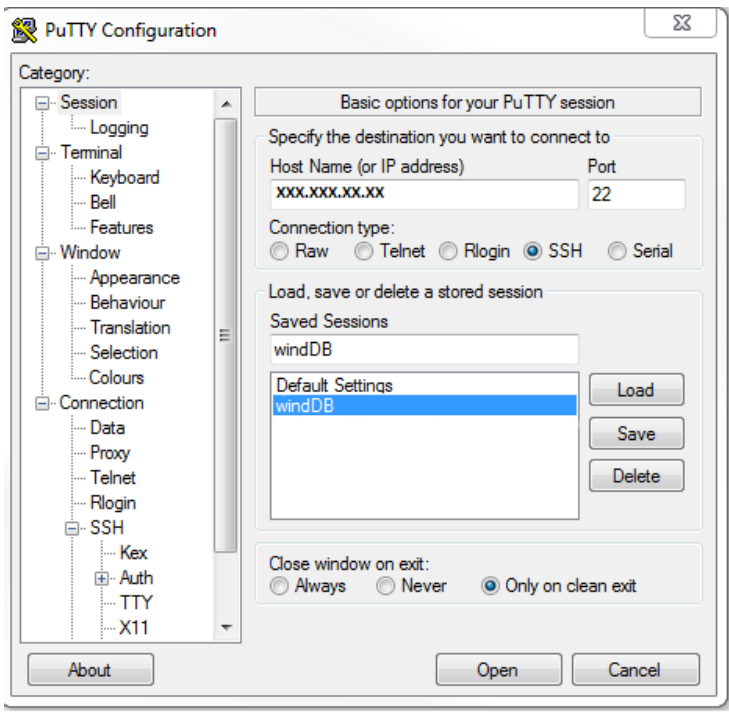

Figure 74: Putty connection setup

To start the tunnel either; open a new terminal and enter uwdTunnel, or open Putty and load the saved windDB configuration, if the client is Linux or Windows respectively. You should be prompted for a user name and/or password, enter your Kumomotojo user details or the provided user specific credentials. Open the tunnel before starting MATLAB and loading the Simulink model and leave the terminal open for the duration of using the simulator.

\section{D.3 Driver Installation}

The connection between MATLAB and MySQL is performed though a collection of java scripts bundled together into a java executable. Unfortunately this requires a driver installation and slight modifications to the MATLAB running on the client computer. The following steps outline downloading the java driver and how to install it, using the platform independent method. 
1. The compressed files are available for download along side this tutorial. Alternatively the same compressed archive can be downloaded from https: //dev.mysql.com/downloads/connector/j/ by selecting the Platform Independent option.

2. After opening the zip file, the mysql-connector-java-5.1.30-bin.jar must be moved to the java folder in root MATLAB directory. From the root MATLAB installation folder, where MATLAB was installed to on the client computer, the file path is $\cdot /$ java/jar/.

3. To tell MATLAB where to look for the drive, a small edit is made in the Java class path. Open MATLAB and type edit classpath.txt in the Command Window. Ignore any autogenterator warnings that may appear.

4. At the very bottom of the edit classpath.txt add the following line: \$matlabroot/java/jar/mysql-connector-java-5.1.30-bin.jar.

After a restart of MATLAB, the connector should allow the data in the MySQL database to be remotely accessed and applied in the flight simulator. Instructions on how to view the contents of the UWB and how to manually query it though MATLAB for testing purposes, followed by the details linking the actual Simulink simulator, are now presented.

\section{D.4 Database Access and MATLAB Connection}

The available database entries can be accessed by using the previously setup SSH connection to the host server. Next log into MySQL using the following command, 
mysql -u userName -p

Where userName is the provided MySQL login name, not your Kumomotojo user name. After pressing Enter you'll be promoted for the provided MySQL password. You should get a welcome screen as shown in Figure 75 and a mysql> command prompt. From here you can view all of the databases stored on the server by entering show databases; again illustrated in Figure 75. To actually access and view the wind data, enter a command such as use windDB; to change into that database.

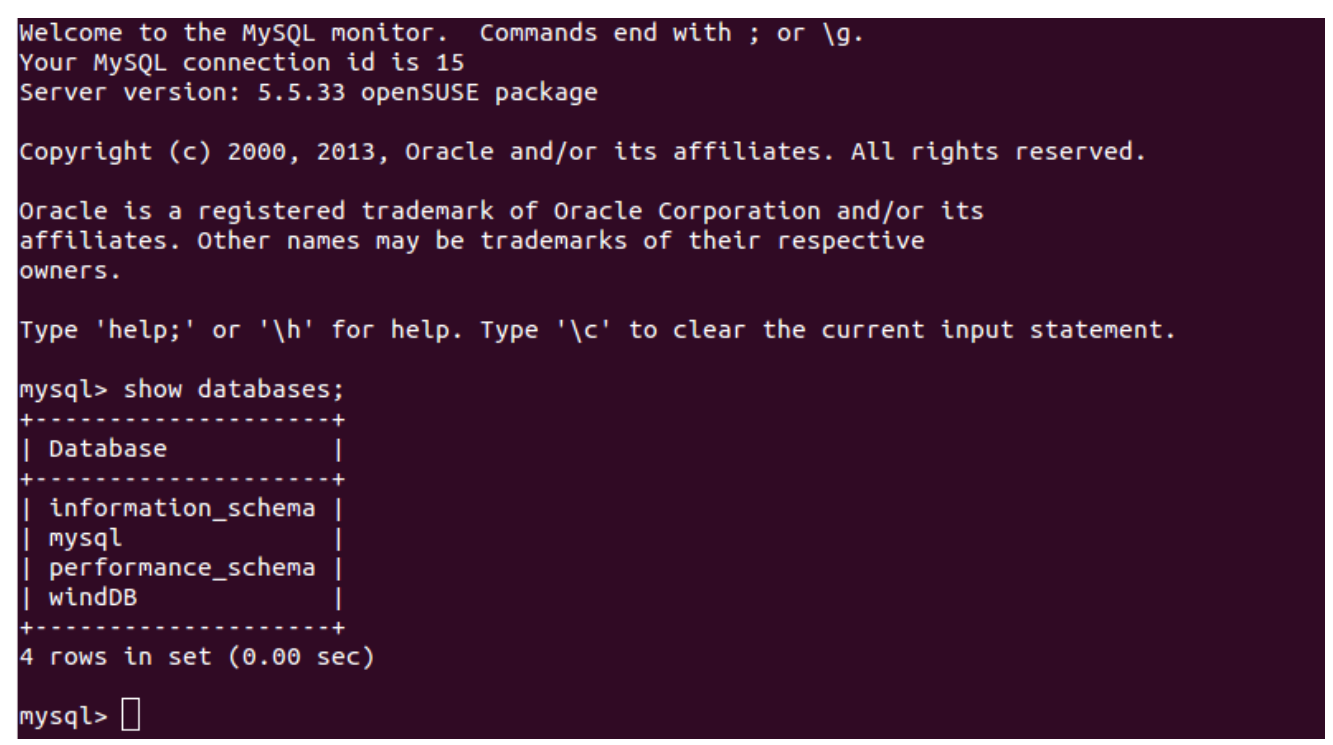

Figure 75: List of all the databases stored on the MySQL server

To list the tables in the windDB for example, use the show tables; command. This will return a huge list of all the tables in the database, a subset is shown in Figure 76. As previously outlined when producing the .csv files, each table represents a saved timestep of the CDF simulations. An appropriate save interval is found to be 0.05 seconds, Section 4.1.1, and loop intervals from the techniques in Section 4.1.2. 


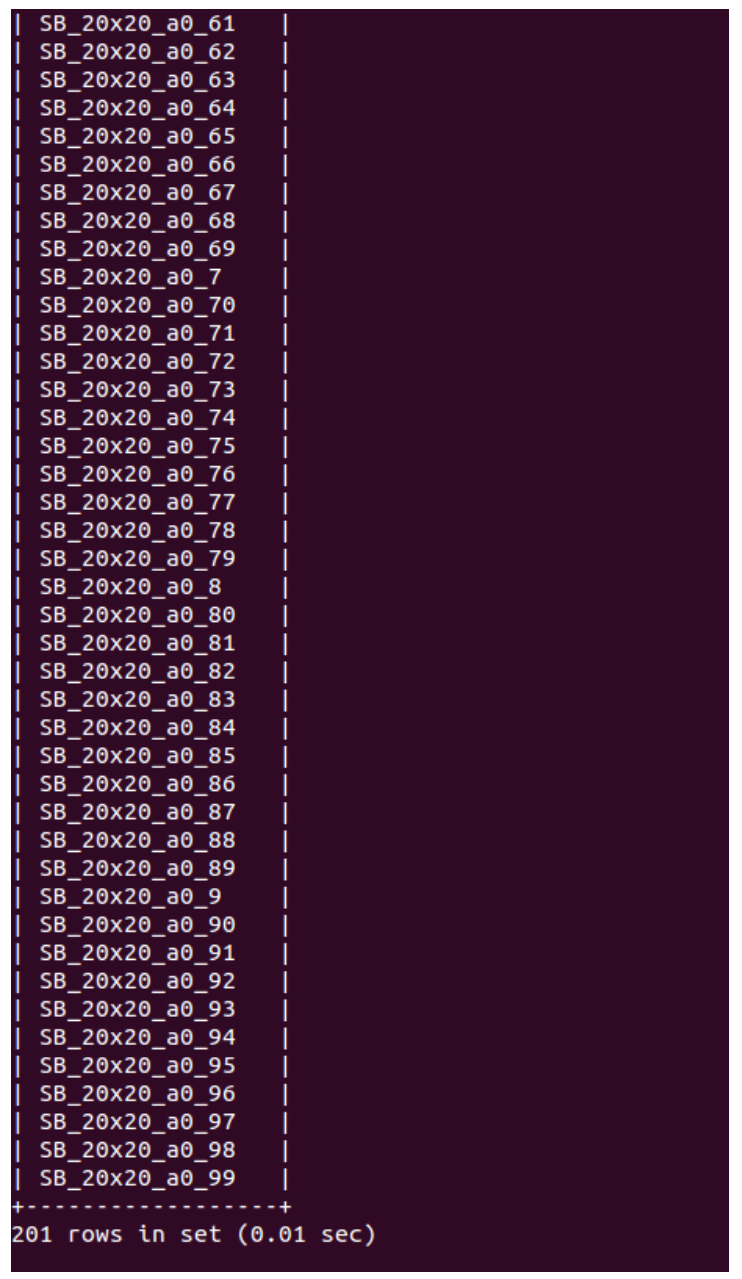

Figure 76: List of tables in windDB

To view the contents of a specific table enter a command such as,

$$
\text { select * from 20x20x120_a0_88 limit } 10 \text {; }
$$

This command will select table 20x20x120_a0_88 and display all the columns and the first 10 rows of the table. The limit should always be used due to the huge volume of stored data in each table, this example has 1,322,025 rows. Figure 77 illustrates the results of the select command along with the structure of the stored data. Each 
point in the flight area $(\mathrm{x}, \mathrm{y}, \mathrm{z})$ has corresponding velocity components and a pressure value.

\begin{tabular}{|c|c|c|c|c|c|c|}
\hline p & I Ux & | Uy & I Uz & $\mid x$ & $\mathrm{y}$ & $\mathrm{z}$ \\
\hline-1.39088 & | 13.8205 & -0.118983 & | -2.98557 & 34.375 & 15 & 4.375 | \\
\hline-1.58209 & 13.8236 & | -0.00188551 & -3.29029 & 34.375 & 15 & 4.0625 \\
\hline-1.82928 & 13.7887 & $\quad-0.113196$ & -3.64142 & 34.375 & 14.6875 & 4.0625 \\
\hline-1.41211 & 13.8576 & -0.184618 & -3.33488 & 34.375 & 14.6875 & 4.375 \\
\hline-4.35744 & 14.1705 & 0.104261 & -4.21018 & 34.375 & 15 & 2.5 \\
\hline-3.81308 & 14.3877 & 0.197164 & -3.17902 & 34.375 & 14.6875 & 2.5 \\
\hline-2.72841 & 14.2313 & 0.0271365 & -3.25001 & 34.375 & 14.6875 & 2.8125 \\
\hline-3.82949 & 14.794 & 0.08555556 & -3.87724 & 34.375 & 15 & 2.8125 \\
\hline-2.70415 & 14.8412 & -0.86827 & -3.77006 & 34.375 & 15 & 3.125 \\
\hline-1.74024 & 13.7604 & -0.771009 & -3.60255 & I 34.375 & 14.6875 & 3.125 \\
\hline
\end{tabular}

Figure 77: Query from the select and limit command $(t=4.4 s)$

To access this data in MATLAB as a test, start the SSH tunnel followed by MATLAB. Normally the dbconnect. $m$ file in the flight simulator handles the database connection and will be setup to automatically connect when the simulator is started. The import parts of the file are used here to manually test the connection. Create a database connection object by entering the following command in the MATLAB Command Window.

$$
\begin{gathered}
\text { conn = database('dbName', 'userName', 'passWord', } \\
\text { 'com.mysql.jdbc.Driver', 'jdbc:mysql://localhost:7800/dbName') }
\end{gathered}
$$

Where dbName is the database name such as windDB, and userName and pass Word are the authorized MySQL login details. Note the name of the database is required twice, at the beginning and end of the command. Then make an execution command by entering, 


$$
\text { curs }=\operatorname{exec}(\operatorname{conn}, \text { 'select } * \text { from 20x20x120_a0_88 limit 10'); }
$$

Actually query the data from the database,

$$
\operatorname{curs}=\text { fetch(curs) }
$$

To visually see the pulled data,

\section{curs.Data}

If there are no error messages at any point and a 10 by 7 matrix should be returned and the SSH tunnel/MATLAB database integration is working. Similarly it should now be possible for a remote client to access the MySQL server at run time to return the urban wake velocities to the flight simulator. 